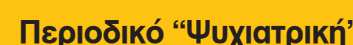

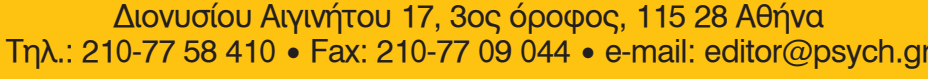
The Journal "Psychiatriki"

17, Dionisiou Eginitou str., 3rd floor, 11528 Athens, Greece
Tel.: (+30) 210-77 $58410 \bullet$ Fax: (+30) 210-210-77 $09044 \bullet$ e-mail: editor@psych.gr

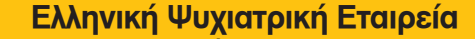

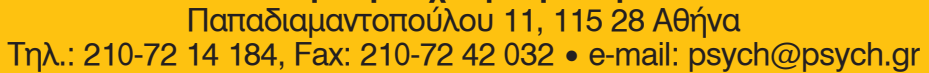

Hellenic Psychiatric Association

11, Papadiamantopoulou stri, 11528 Athens, Greece
Tel.: (+30) 210-72 14 184, Fax: (+30) 210-72 42032 e-mail: psych@psych.gr
WYXIATPIKH

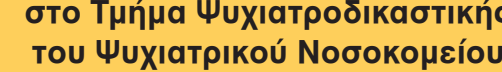
WYXIATPIKH
UYXIATPIKH
UYTPIKH

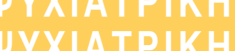
PYXIATPIKH WYXIATPIKH PYXIATPIKH

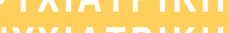
TAMATPIKH WXXAATPIKH
UYXIATPIKH UYXIATPIKH WYXIATPIKH
UYXIATPIKH UYXIATPIKH UYXIATPIKH WYXIATPIKH WYXIATPIKH WYXIATPIKH WYXIATPIKH PYXIATPIKH PYXIATPIKH PYXIATPIKH WYXIATPIKH WYXIATPIKH WYXIATPIKH WYXIATPIKH UYXIATPIKH PYXIATPIKH PYXIATPIKH WXIATPIKH UYXIATPIKH PYXIATPIKH UYXIATPIKH PYXIATPIKH
UVYIATPIKH WYXIATPIKH
WVYIATPIKH WYXIATPIKH
WVXIATPIKH DYXIATPIKH
UYXIATPIKH DYXIATPIKH
WYXIATPIKH UYXIATPIKH WYXIATPIKH WYXIATPIKH WYXIATPIKH WYXIATPIKH UYXIATPIKH

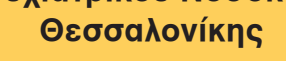

М. Маркопои́хоu каı бuv $\bullet \bullet \bullet$

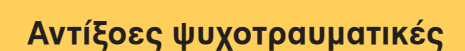

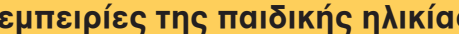

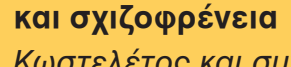

$$
\text { -.. }
$$

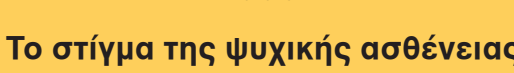

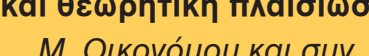

$$
\bullet \bullet
$$

Avoco Kal aUtoávoool $\mu \eta$ Xavionoí

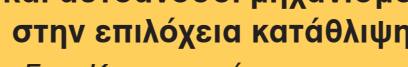
$\lceil\varepsilon \rho$. Kwvotavtívou kal ouv $\bullet \bullet$

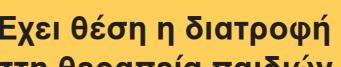

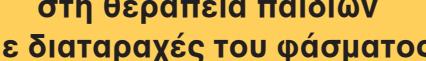
Tou autionoú; M. Ѓ́you \& Г. Koגı́s -..•

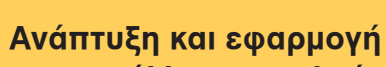

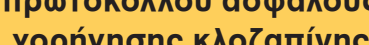

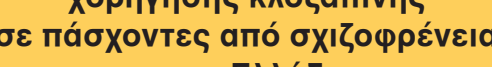

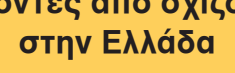

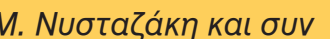

•.•

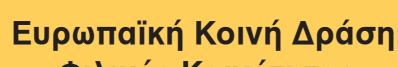

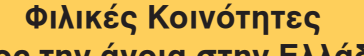
A. Пoגítns kal ouv

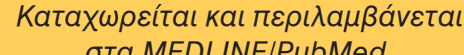
Index Copernicus, Scopus, Google Scholar, EMBASE/Excerpta for Scientific Inf., EBSCOhost t" PsychINFO kal oto latrotek,
(Scopus CiteScore 2018 $=0.62$ ) Ioторіки́ avadpopŕ TO

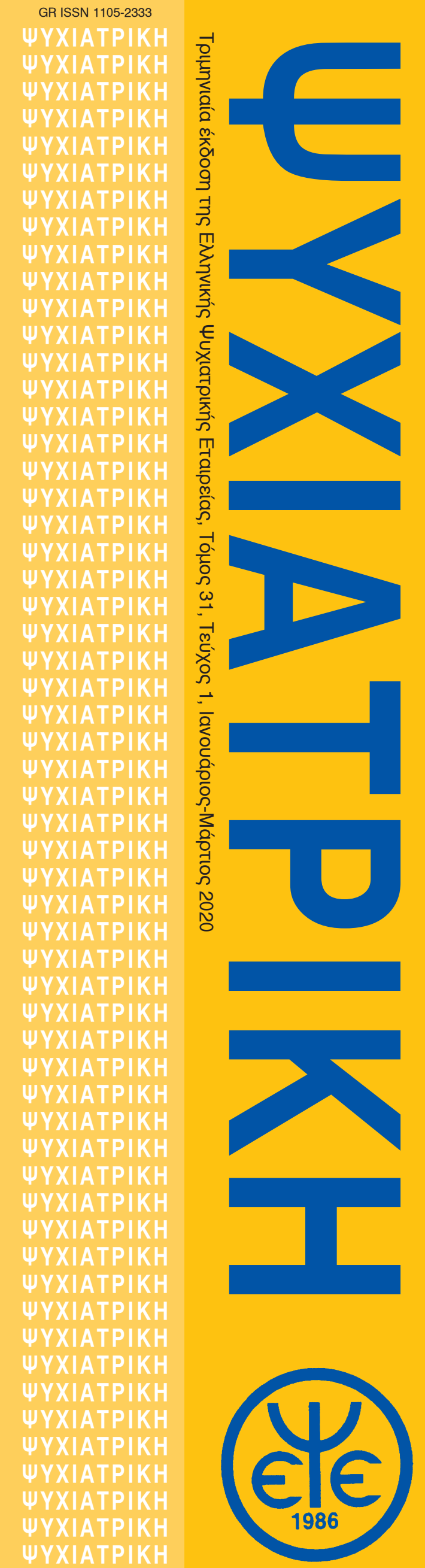




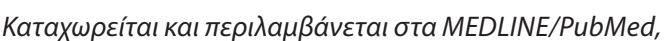
Index Copernicus, Scopus, Google Scholar, EMBASE/Excerpta Medica, GFMER, CIRRIE, SCIRUS for Scientific Inf., EBSCOhost ${ }^{T M}$, PsychINFO Kaı бто latrotek

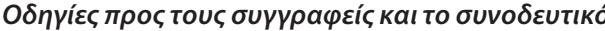

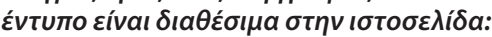
http://www.psychiatriki-journal.gr

\section{YYXIATPIKH}

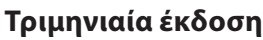

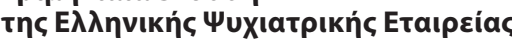

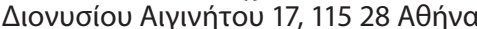

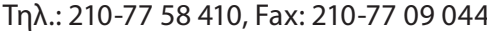

Екठо́тnc:

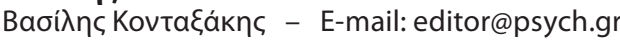

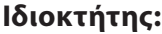

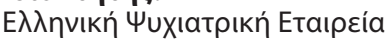

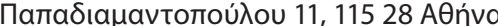

$\operatorname{T\eta } \lambda .:$ 210-72 14184

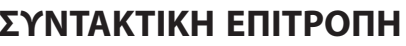

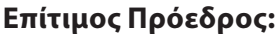

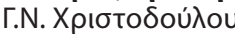

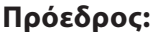

B. Kovta\}áknc

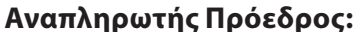

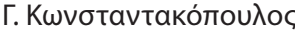

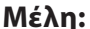

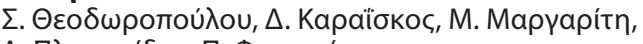

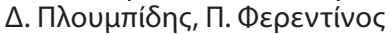

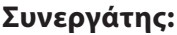

I. Zźpßac

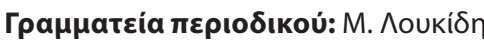

Indexed and included in MEDLINE/PubMed, Index Copernicus, Scopus, Google Scholar, EMBASE/Excerpto Medica, GFMER, CIRRIE, SCIRUS for Scientific Inf., EBSCOhost ${ }^{T M}$, PsychINFO and in latrotek

Instructions to contributors and the submission form are available at the webpage

http://www.psychiatriki-journal.gr

\section{PSYCHIATRIKI}

Quarterly journal published

by the Hellenic Psychiatric Association

17, Dionisiou Eginitou str., 11528 Athens

Tel.: +30-210-77 58 410, Fax: +30-210-77 09044

Publisher:

Vassilis Kontaxakis - E-mail: editor@psych.gr

Owner:

Hellenic Psychiatric Association

11, Papadiamantopoulou str., 11528 Athens

Tel.: +30-210-72 14184

\section{EDITORIAL BOARD}

Emeritus Editor:

G.N. Christodoulou

Editor-in-Chief:

V. Kontaxakis

Associate Editor:

G. Konstantakopoulos

Members:

S. Theodoropoulou, D. Karaiskos, M. Margariti,

D. Ploumpidis, P. Ferentinos

Collaborator:

J. Zervas

Journal's secretariat: M. Loukidi

$\begin{array}{ll}\text { M. Abou-Saleh (UK) } & { }^{\dagger} \text { H. Ghodse (UK) } \\ \text { H. Akiskal (USA) } & \text { P. Gökalp (Turkey) } \\ \text { G. Alexopoulos (USA) } & \text { G. Ikkos (UK) } \\ \text { N. Andreasen (USA) } & \text { R.A. Kallivayalil (India) } \\ \text { S. Bloch (Australia) } & \text { M. Kastrup (Denmark) } \\ \text { M. Botbol (France) } & \text { K. Kirby (Australia) } \\ \text { N. Bouras (UK) } & \text { V. Krasnov (Russia) }\end{array}$

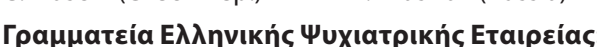

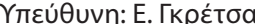

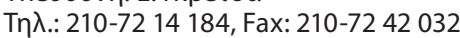

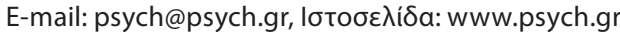
FB: E^^HNIKH $\Psi$ YXIATPIKH ETAIPEIA

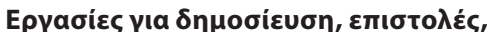

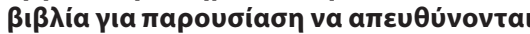

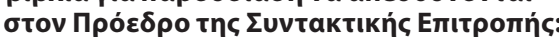
Aıvuoíou Aıүıvńtou 17, 11528 AӨńva

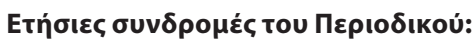

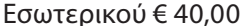

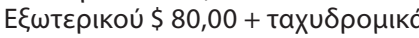

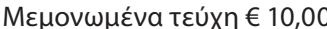

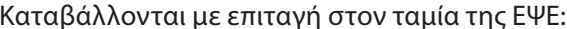

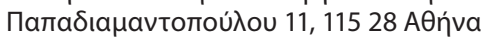

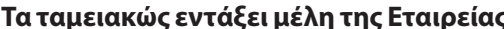

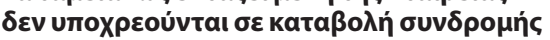

\section{EПIMEAEIA EK $\Delta O \Sigma \mathrm{H} \Sigma$} EN ISO 9001:2000

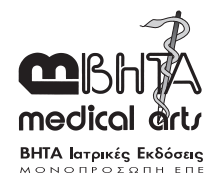

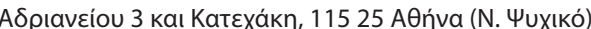
Tn入.: 210-67 14371 - 210-67 14 340, Fax: 210-67 15015 e-mail: betamedarts@otenet.gr e-shop: www.betamedarts.gr

EN ISO 9001:2000

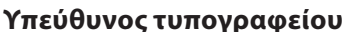

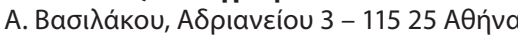

$\operatorname{T\eta } \lambda .210-6714340$
D. Lecic-Tosevski (Serbia) G. Papakostas (USA)

C. Lyketsos (USA)

G. Petrides (USA)
R. Salokangas (Finland)

M. Maj (Italy)

A. Marneros (Germany)

J. Mezzich (USA)

H.J. Möller (Germany) A. Tasman (USA)

O. Steenfeld-Foss (Norway)

R. Montenegro (Argentina) N. Tataru (Romania)

C. Pantelis (Australia) P. Tyrer (UK)

Secretariat of Hellenic Psychiatric Association: Head: H. Gretsa

Tel.: (+30) 210-72 14 184, Fax: (+30) 210-72 42032

E-mail: psych@psych.gr,Web-site:www.psych.gr

Manuscripts, letters, books for review should be addressed to the Editor:

17 Dionisiou Eginitou str., GR-115 28 Athens, Greece

Annual subscriptions of the Journal:

$€ 40.00$ or $\$ 80.00$ + postage - each separate issue $€ 10.00$ are payable by check to the treasurer

of the Hellenic Psychiatric Association:

11, Papadiamantopoulou str., GR-115 28 Athens

For the members of the Association in good standing subscription is free

\section{EDITING}

EN ISO 9001:2000

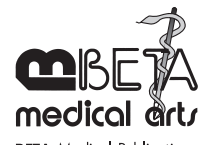

3. Adrianiou str, GR-115 25 Athens-Greece

Tel.: (+30) 210-67 14371 - (+30) 210-67 14340

Fax: (+30) 210-67 15015

e-mail: betamedarts@otenet.gr, e-shop:www.betamedarts.gr EN ISO 9001:2000

Printing supervision

A. Vassilakou, 3 Adrianiou str. - GR-115 25 Athens

Tel. (+30)-210-67 14340 
EAMHNIKH YYXIATPIKH ETAIPEIA

\section{DIOIKHTIKO EYMBOYAIO}

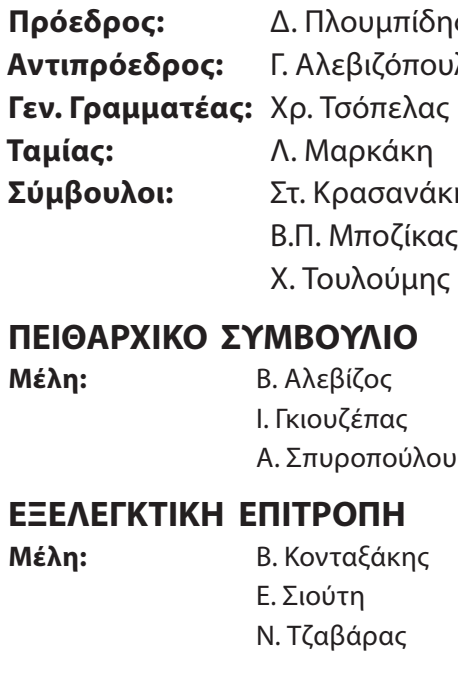

EחITIMOI ПPOE $\triangle$ POI

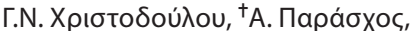

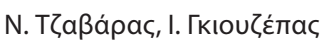

\section{EПITIMA MEAH}

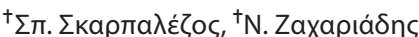

†. Пıттарác, X. Bapouxáknৎ*

\begin{tabular}{|c|c|}
\hline \multicolumn{2}{|c|}{ ПЕРІФЕРЕІАКА ТМНМАТА } \\
\hline 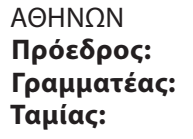 & 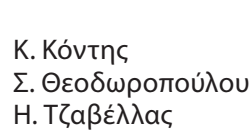 \\
\hline 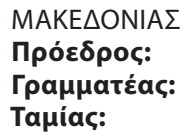 & 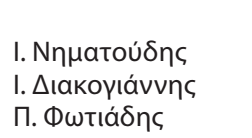 \\
\hline 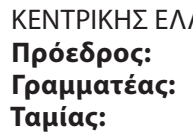 & 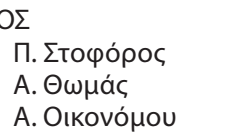 \\
\hline
\end{tabular}

BOPEIO $\triangle$ YTIKH $\Sigma$ E $\wedge$ A $\triangle \mathrm{O} \Sigma \&$ \& ҮTIKH $\Sigma \Sigma T$ TEPEA $\Sigma$

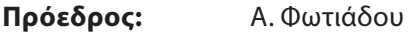

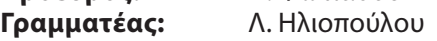

Tauiac: $\quad$ П. Пвтрі́кns

ПEЛOПONNHEOY

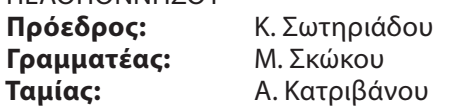

MEГA^H $\Sigma$ BPETTANIA $\Sigma$

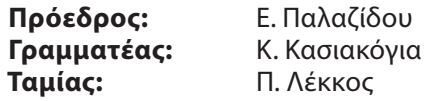

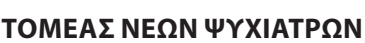

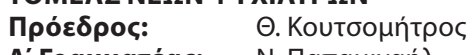

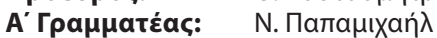

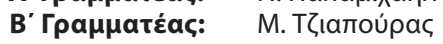

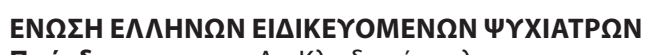

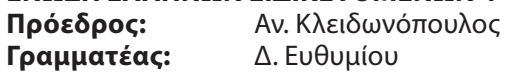

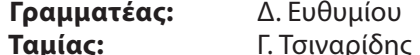

\section{HELLENIC PSYCHIATRIC ASSOCIATION}

\section{EXECUTIVE COUNCIL}

\author{
Chairman: D. Ploumpidis \\ Vice-Chairman: G. Alevizopoulos \\ Secretary General: Ch. Tsopelas \\ Treasurer: L. Markaki \\ Consultants: St. Krasanakis \\ V.P. Bozikas \\ Ch. Touloumis

\section{DISCIPLINARY COUNCIL

$\begin{array}{ll}\text { Members: } & \text { V. Alevizos } \\ & \text { J.Giouzepas } \\ & \text { A. Spyropoulou }\end{array}$

FINANCIAL CONTROL COMMITTEE

$\begin{array}{ll}\text { Members: } & \text { V. Kontaxakis } \\ & \text { Ir. Siouti } \\ & \text { N. Tzavaras }\end{array}$

HONORARY PRESIDENTS

G.N. Christodoulou, ${ }^{\dagger}$ A. Paraschos, N. Tzavaras, J. Giouzepas

\section{HONORARY MEMBERS}

${ }^{\dagger} \mathrm{S}$. Scarpalezos, ${ }^{\dagger} \mathrm{N}$. Zachariadis,

${ }^{\dagger}$ I. Pittaras, Ch. Varouchakis*

\section{DIVISIONS}

ATHENS

\begin{tabular}{|c|c|}
\hline $\begin{array}{l}\text { Chairman: } \\
\text { Secretary: } \\
\text { Treasurer: }\end{array}$ & $\begin{array}{l}\text { C. Kontis } \\
\text { S. Theodoropoulou } \\
\text { E. Tzavellas }\end{array}$ \\
\hline $\begin{array}{l}\text { MACEDONIA } \\
\text { Chairman: } \\
\text { Secretary: } \\
\text { Treasurer: }\end{array}$ & $\begin{array}{l}\text { J. Nimatoudis } \\
\text { J. Diakoyiannis } \\
\text { P. Fotiadis }\end{array}$ \\
\hline $\begin{array}{l}\text { CENTRAL GREECE } \\
\text { Chairman: } \\
\text { Secretary: } \\
\text { Treasurer: }\end{array}$ & $\begin{array}{l}\text { P. Stoforos } \\
\text { A. Thomas } \\
\text { A. Oikonomou }\end{array}$ \\
\hline
\end{tabular}

NORTHWESTERN GREECE

$\begin{array}{ll}\begin{array}{l}\text { Chairman: } \\ \text { Secretary: }\end{array} & \text { A. Fotiadou } \\ \text { Treasurer: } & \text { P. lliopoulou } \\ \text { PELOPONNESE } & \\ \text { Chairman: } & \text { K. Sotiriadou } \\ \text { Secretary: } & \text { M. Skokou } \\ \text { Treasurer: } & \text { A. Katrivanou } \\ \text { GREAT BRITAIN } & \\ \text { Chairman: } & \text { H. Palazidou } \\ \text { Secretary: } & \text { K. Kasiakogia } \\ \text { Treasurer: } & \text { P. Lekkos }\end{array}$

SECTOR OF YOUNG PSYCHIATRISTS

Chairman: Th. Koutsomitros

Secretary A': N. Papamichael

Secretary B': $\quad$ M. Tziapouras

UNION OF GREEK PSYCHIATRIC TRAINEES

$\begin{array}{ll}\text { Chairman: } & \text { A. Kleidonopoulos } \\ \text { Secretary: } & \text { D. Efthymiou }\end{array}$

$\begin{array}{ll}\text { Secretary: } & \text { D. Efthymiou } \\ \text { Treasurer: } & \text { G. Tsinaridis }\end{array}$ 


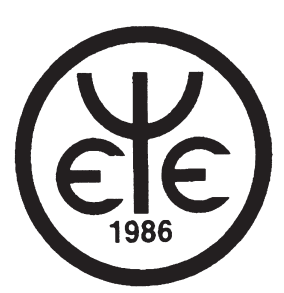

KAA $\triangle O$ O

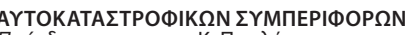

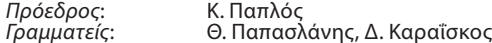

BIAISN $\Sigma Y M T E P I \Phi O P \Omega N$

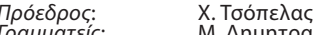

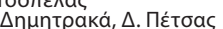

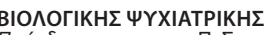

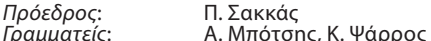

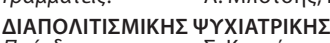

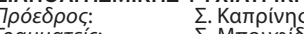

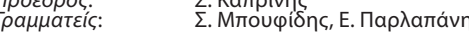

$\triangle$ IATAPAXE

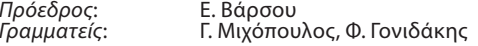

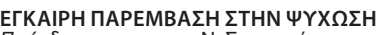

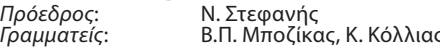

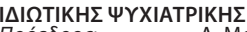

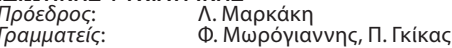

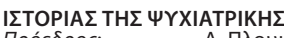

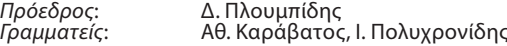

KAINIIKH YYXOФAPMAKONOIIAE

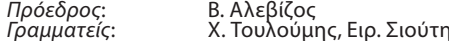

KOINSNIKHE $\Psi$ YXIATPIKH $\Sigma$

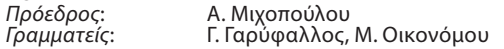

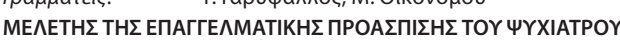

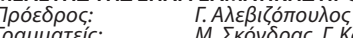

Г Карантоита́кпऽ

NEYPOANAחTYEIAKEE $\triangle$ IATAPAXEE $\triangle I A$ BIOY

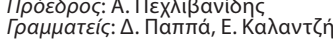

OY $\Sigma I O E E A P T H L E \Omega N$

I. Sıakoyı́ávn

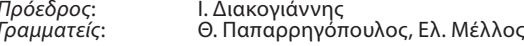

TAI $\triangle O \Psi Y X I A T P I K H \Sigma$

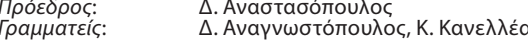

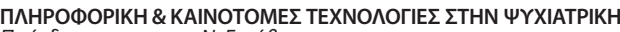

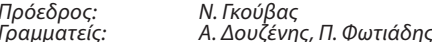

ПРОЛНПTIКH $\Psi$ YXIATPIKH $\Sigma$

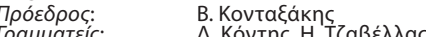

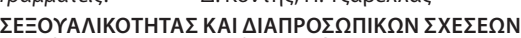

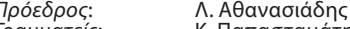

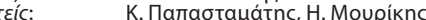

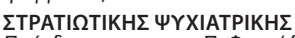

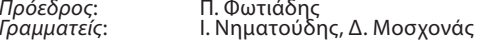

¿YMBOYAEYTIKHE- $\triangle$ IAIYNAETIKH

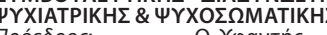

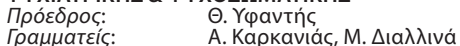

TEXNH $\Sigma$ \& $\Psi$ YXIATPIKH $\Sigma$

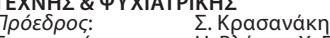

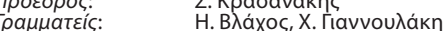

THAEYYXIATPIKH $\Sigma$

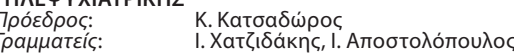

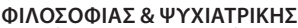

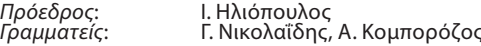

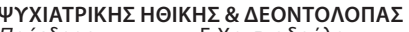

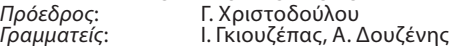

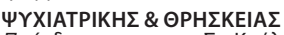

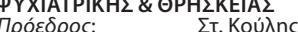

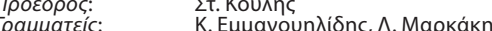

YYXIKH Y YTEIAE TYNAIK $\Omega N \&$

YYXIATPIKH $\Sigma$ TH $\Sigma$ ANATAPATOTH

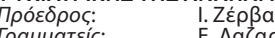

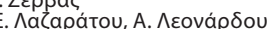

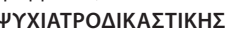

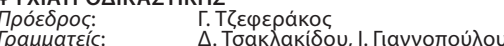

YYXOOEPATEIAL

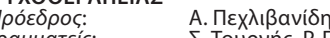

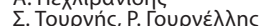

$Y$ YXOГHPIATPIKH

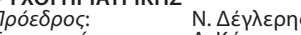

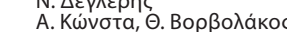

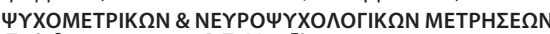

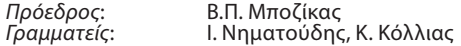

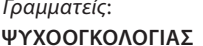

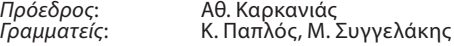

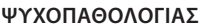

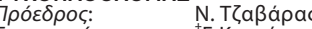

Гранцатві́:

4YXO

Г. Kampivnc, M. $\Delta ı a \lambda \lambda ı$ ivá

Гроворос:

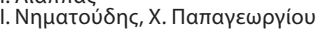

SECTIONS

SELF-DESTRUCTIVE BEHAVIORS

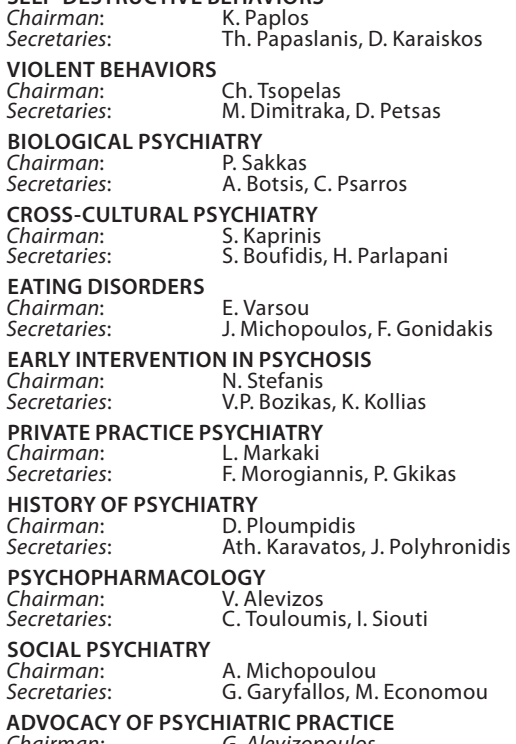

$\begin{array}{ll}\text { Chairman: } & \text { G. Alevizopoulos } \\ \text { Secretaries: } & \text { M. Skondras, G. Karampoutakis }\end{array}$

NEURODEVELOPMENTAL DISORDERS ACROSS THE LIFESPAN

Chairman: A. Pechlivanidis

Secretaries: D. Pappa, E. Kalantzi

SUBSTANCE ABUSE

$\begin{array}{ll}\text { Chairman: } & \text { J. Diakoyiannis } \\ \text { Secretaries: } & \text { Th. Paparrigopoulos, El. Mellos }\end{array}$

CHILD PSYCHIATRY

INFORMATICS \& INNOVATIVE TECHNOLOGIES IN PSYCHIATRY

Chairman:

A. Douzenis, P. Fotiadis

PREVENTIVE PSYCHIATRY

Chairman: $\quad$ V. Kontaxakis

SEXUALITY AND INTERPERSONAL RELATIONSHIPS

$\begin{array}{ll}\text { Chairman: } & \text { L. Athanasiadis } \\ \text { Secretaries: } & \text { K. Papastamatis, H. Mourikis }\end{array}$

MILITARY PSYCHIATRY

Chairman: P. Fotiadis

J.Nimatoudis, D. Moschonas

CONSULTATION-LIAISON PSYCHIATRY

\& PSYCHOSOMATICS

$\begin{array}{ll}\text { Chairman: } & \text { T. Hyphantis } \\ \text { Secretaries: } & \text { A. Karkanias, M. Diallina }\end{array}$

ART \& PSYCHIATRY

Chairman: $\quad S$. Krasanakis

E. Vlasanos, C. Giannoulaki

TELEPSYCHIATRY

Chairman: $\quad$ K. Katsadoros

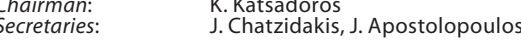

PHILOSOPHY \& PSYCHIATRY

$\begin{array}{ll}\text { Chairman: } & \text { G. Nikolaidis, A. Komborozos }\end{array}$

PSYCHIATRY \& ETHICS

$\begin{array}{ll}\text { Chairman: } & \text { G. Christodoulou } \\ \text { Secretaries: } & \text { J.Giouzepas, A. Douzenis }\end{array}$

PSYCHIATRY \& RELIGION

$\begin{array}{ll}\text { Chairman: } & \text { S. Koulis } \\ \text { Secretaries: } & \text { K. Emmanouilidis, L. Markaki }\end{array}$

WOMEN'S MENTAL HEALTH \&

REPRODUCTIVE PSYCHIATRY

Chairman: $\quad$ Jervas

FORENSIC PSYCHIATRY

FORENSIC PSYCHIATRY
Chairman:

Chairman: $\quad$ G. Tzeferakos

PSYCHOTHERAPY

$\begin{array}{ll}\text { Chairman: } & \text { A. Pechlivanidis } \\ \text { Secretaries: } & \text { S. Tournis, R. Gournellis }\end{array}$

PSYCHOGERIATRICS

Chairman:

Secretaries: $\quad$ A. Kogleris

PSYCHOMETRIC \& NEUROPSYCHOLOGICAL MEASUREMENTS

Chairman: V.P. Bozikas

Secretaries: J. Nimatoudis, K. Kollias

PSYCHO-ONCOLOGY

$\begin{array}{ll}\text { Chairman: } & \text { A. Karkanias } \\ \text { Secretaries: } & \text { K. Paplos, M. Syngelakis }\end{array}$

PSYCHOPATHOLOGY

N.Tzavaras

PSYCHOPHYSIOLOGY

Chairman:

Secretaries: $\quad$ J. Nimatoudis, C. Papageorgiou 


\section{(⿶凵)}

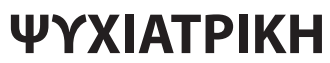

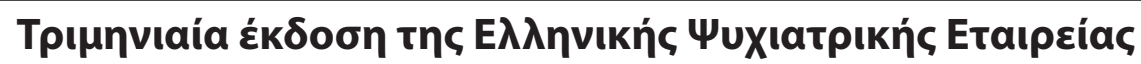

\section{ПEPIEXOMENA}

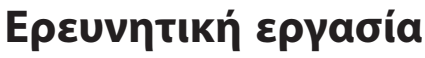

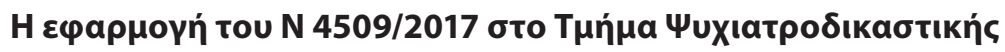

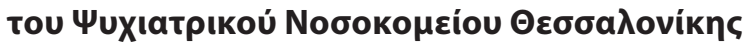

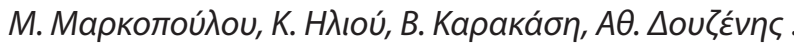

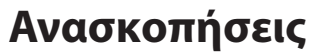

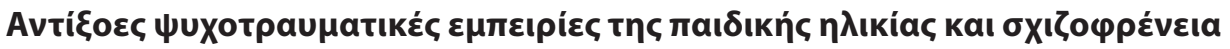

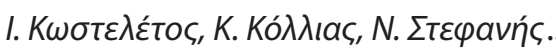

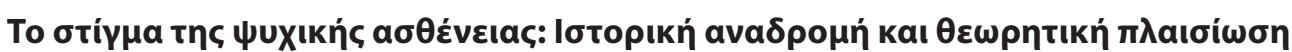

М. Оıкоvó

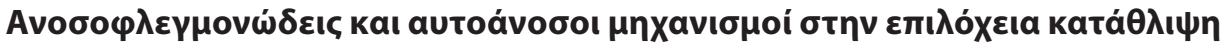

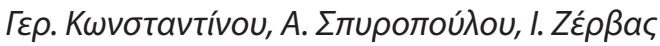

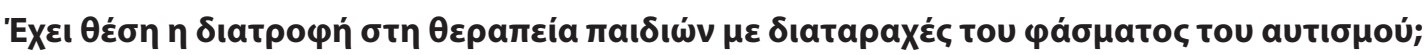

M. Гúyou, Г. Koגıós

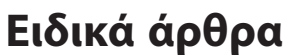

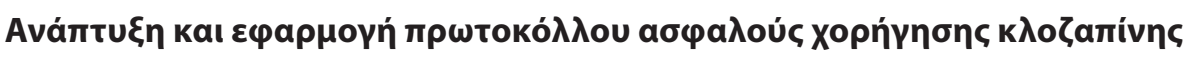

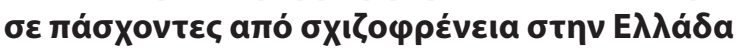

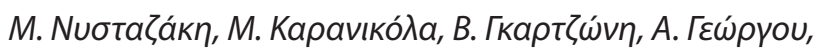

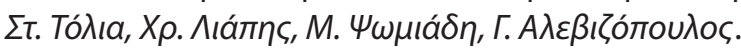

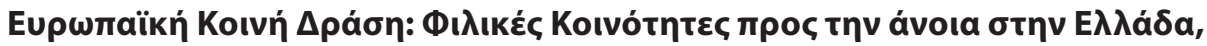

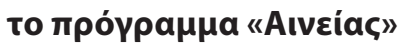

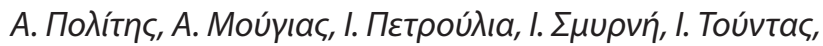

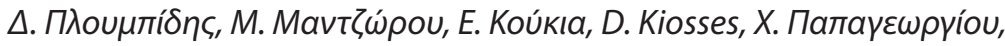

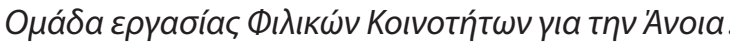




\section{(46)}

\section{PSYCHIATRIKI}

\section{Quarterly journal published by the Hellenic Psychiatric Association}

\section{CONTENTS}

\section{Research article}

The implementation of the law 4509/2017 in the Forensic Department of the Psychiatric Hospital of Thessaloniki

M. Markopoulou, K. Iliou, V. Karakasi, Ath. Douzenis .....

\section{Reviews}

Childhood adverse traumatic experiences and schizophrenia

I. Kosteletos, K. Kollias, N. Stefanis

The stigma of mental illness: A historical overview and conceptual approaches

M. Economou, A. Bechraki, M. Charitsi

Immune-inflammatory and autoimmune mechanisms in postpartum depression

Ger. Konstantinou, A. Spyropoulou, I. Zervas.

Is there place for nutrition in the treatment of children with autism spectrum disorder?

M. Gogou, G. Kolios.

\section{Special articles}

Development and implementation of clozapine protocol

in patients with schizophrenia in Greece

M. Nystazaki, M. Karanikola, V. Gartzoni, A. Georgou,

St. Tolia, Chr. Liapis, M. Psomiadi, G. Alevizopoulos

European Join Action on Dementia: Dementia Friendly Communities in Greece (DFC), the project "Aeneas"

A. Politis, A. Mougias, I. Petroulia, I. Smyrni, I. Tountas,

D. Ploumpidis, M. Mantzorou, E. Koukia, D. Kiosses, Ch. Papageorgiou,

DFC Working group 


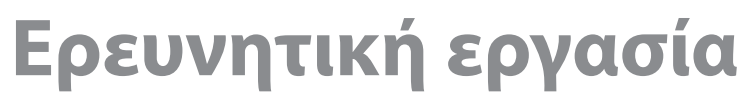 Research article
}

\section{H eсрарноүи́ tou N 4509/2017

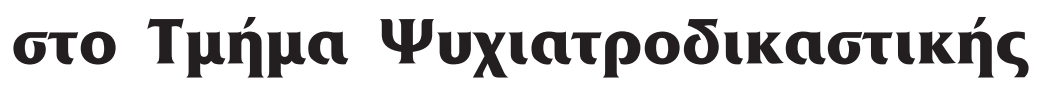

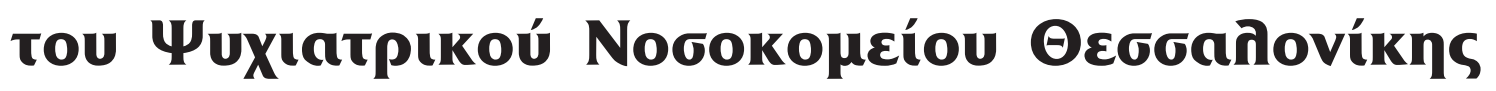

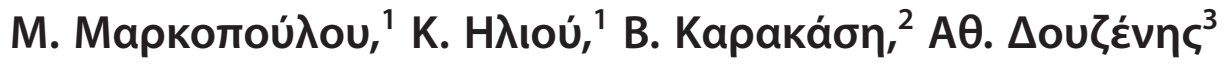

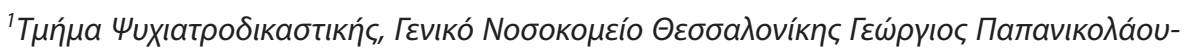

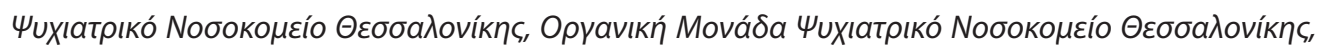

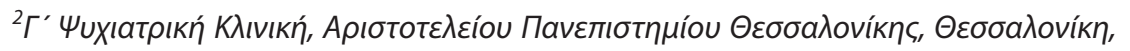

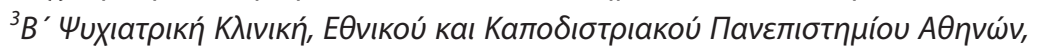

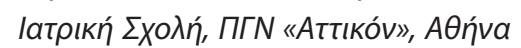

Uuxıатрıкń 2020, 31:13-22

$\sum$

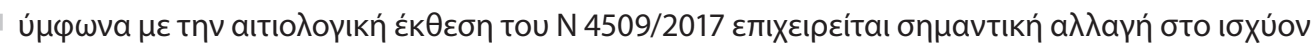

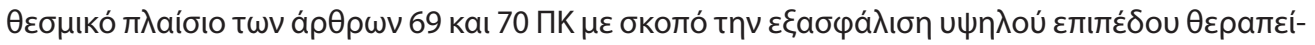

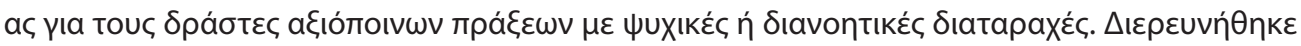

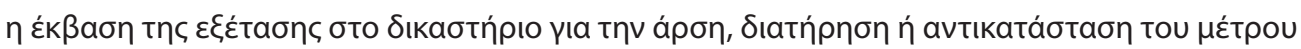
Ө

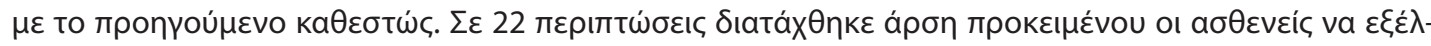

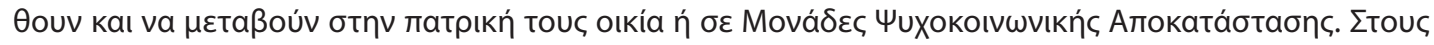

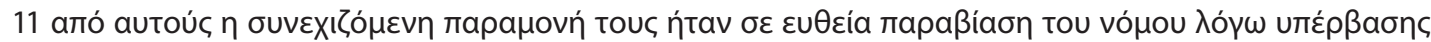

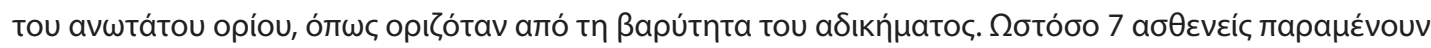

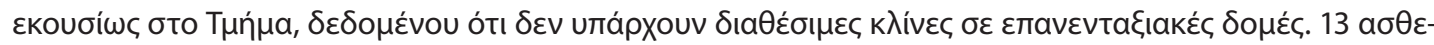

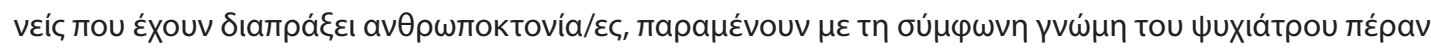

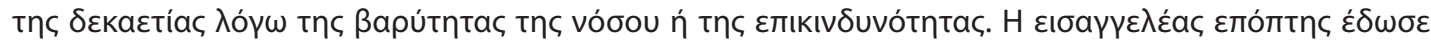

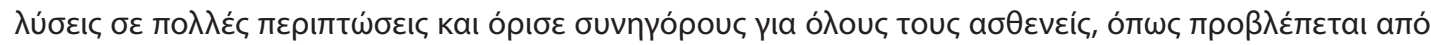

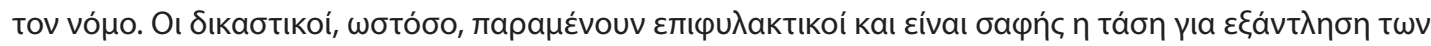

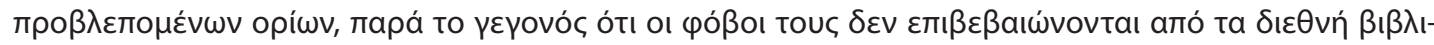

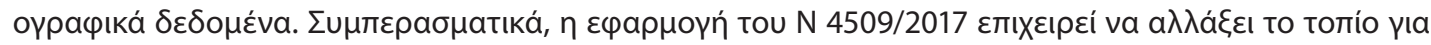

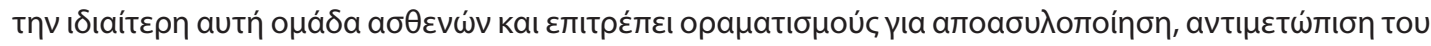

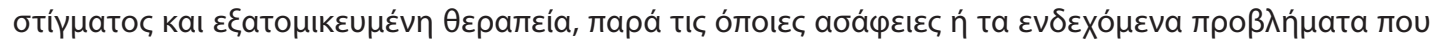

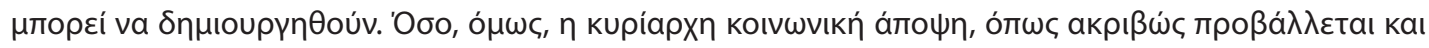




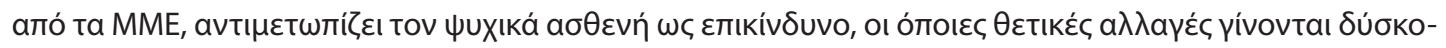

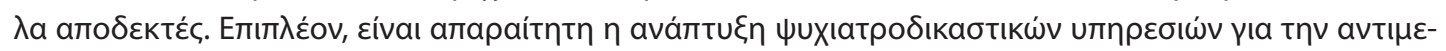

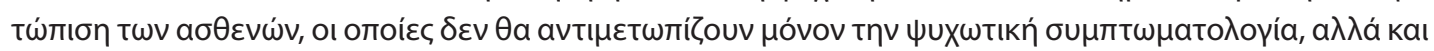

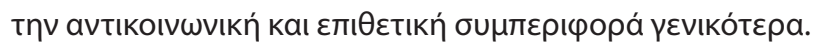

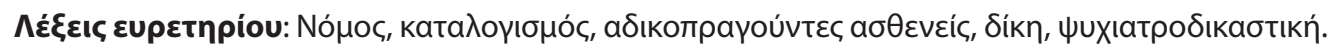

\section{Eıaaywyń}

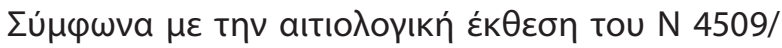

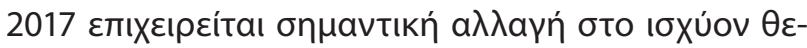

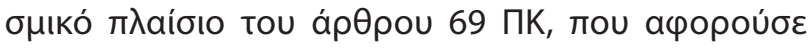

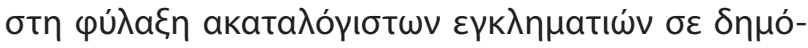

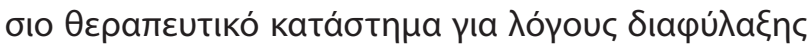

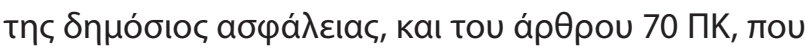

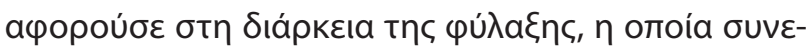

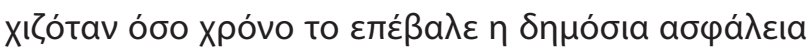

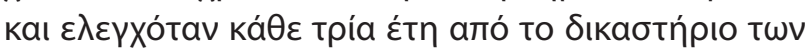

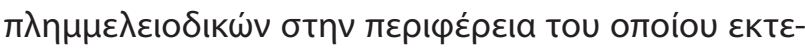

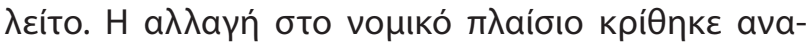

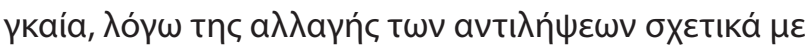

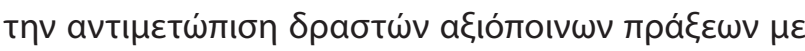

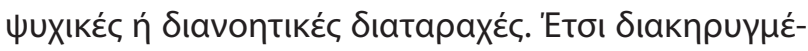

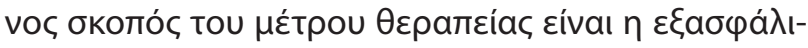

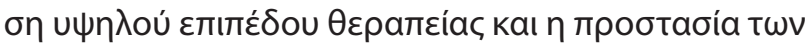

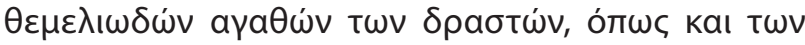

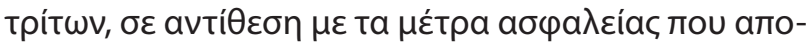

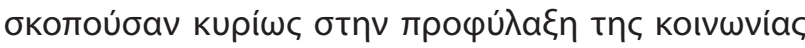

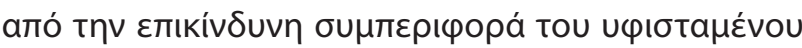
то $\mu$ र́тро.

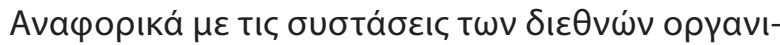

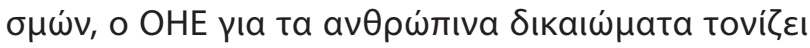

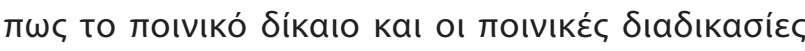

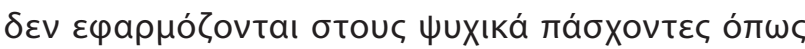

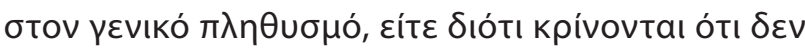

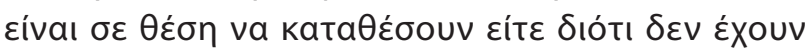

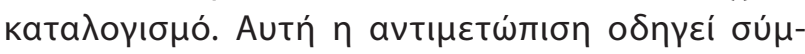

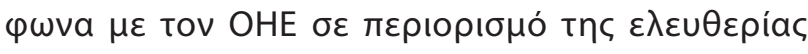

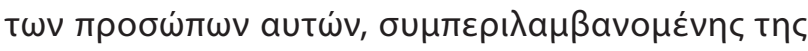

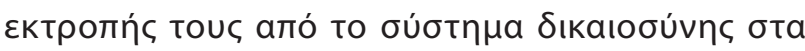

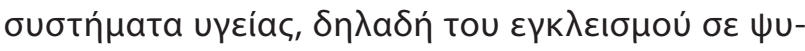

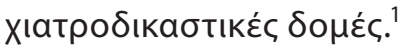

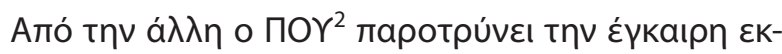

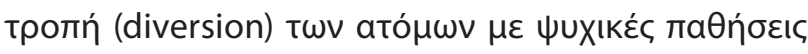

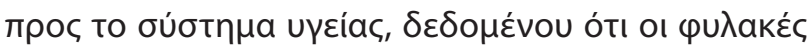

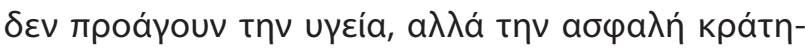

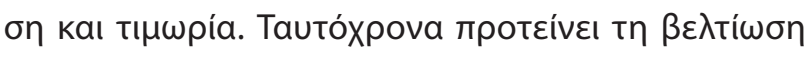

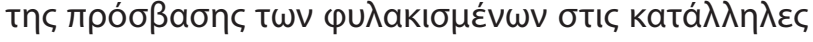

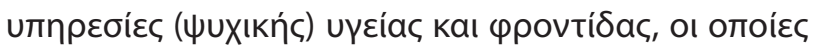

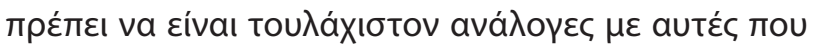

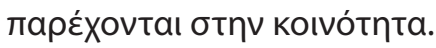

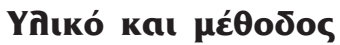

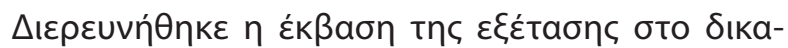

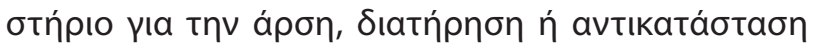

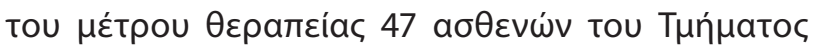

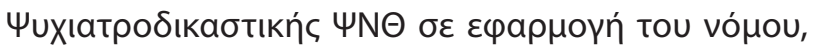

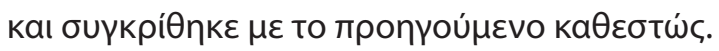

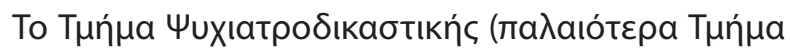

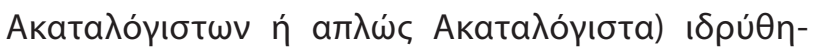

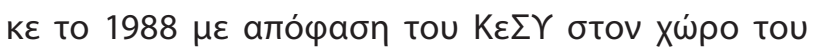

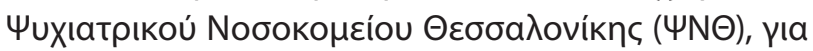

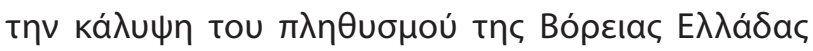
X

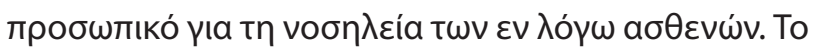

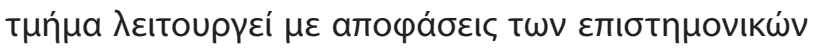

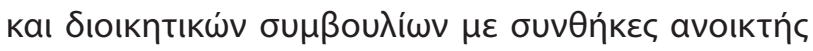

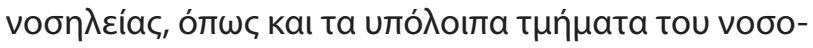
кoukíou.

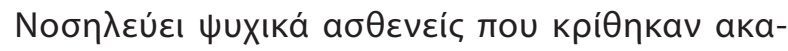

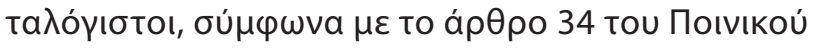

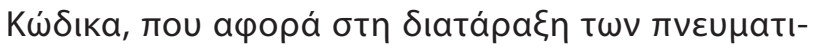

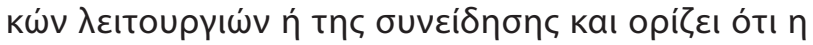

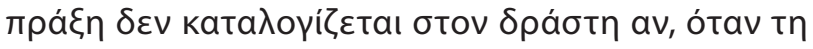

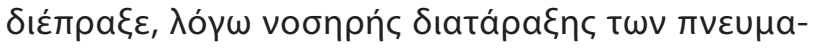

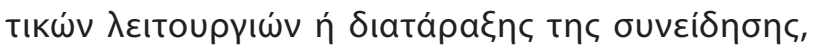

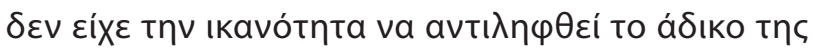

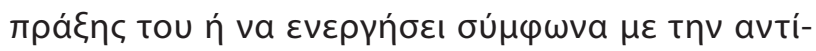

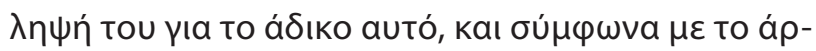

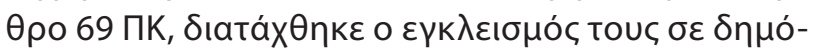

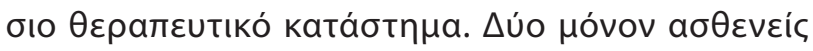

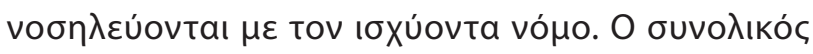

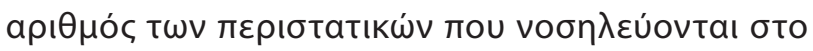

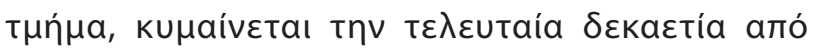

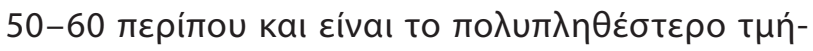
$\mu$ то т $\Psi$ NO. 


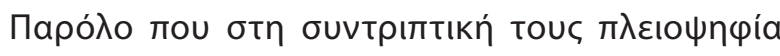

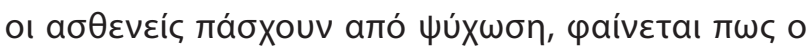

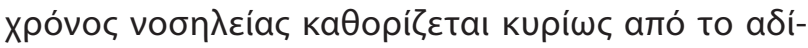

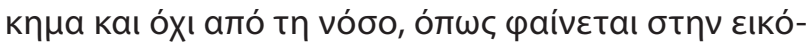

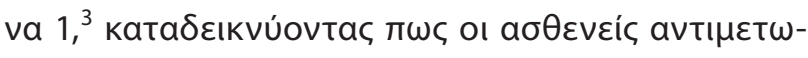

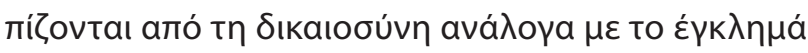

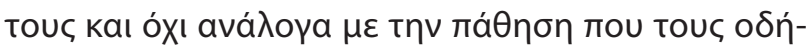

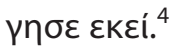

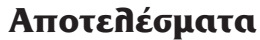

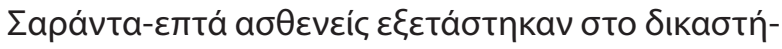

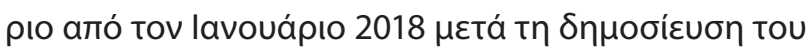

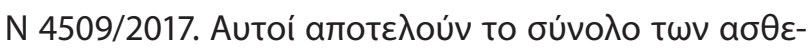

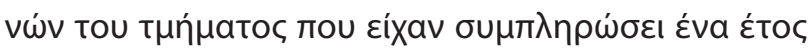

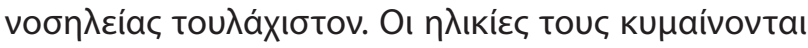

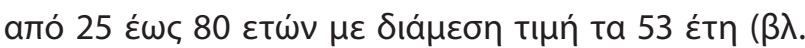

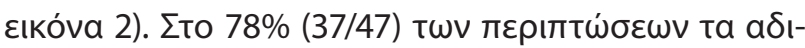

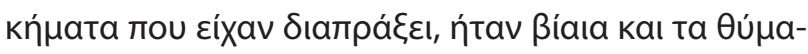

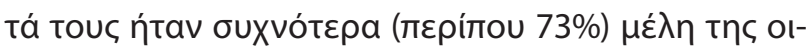

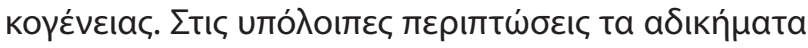
a

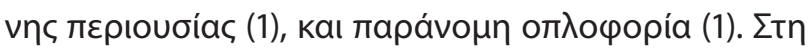

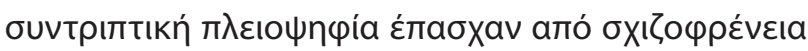

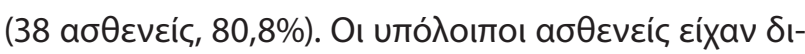

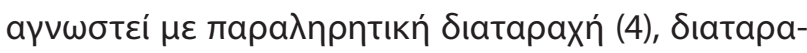

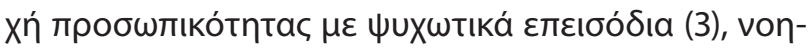

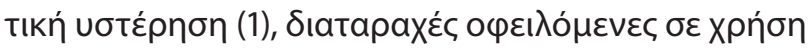
àкоóג (1).

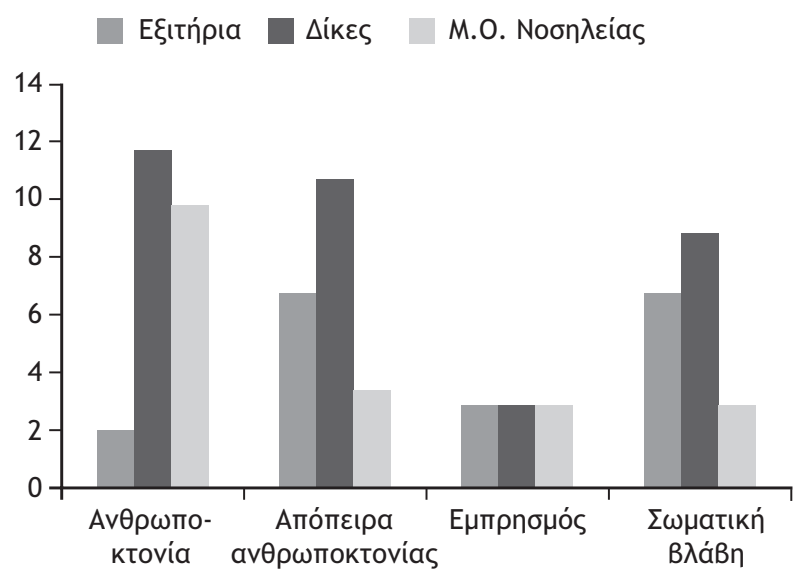

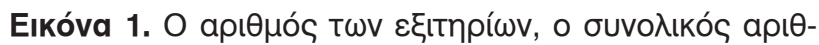

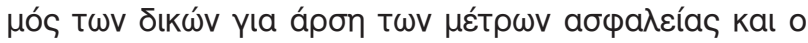

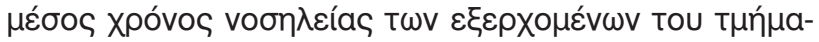

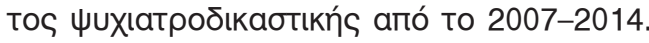

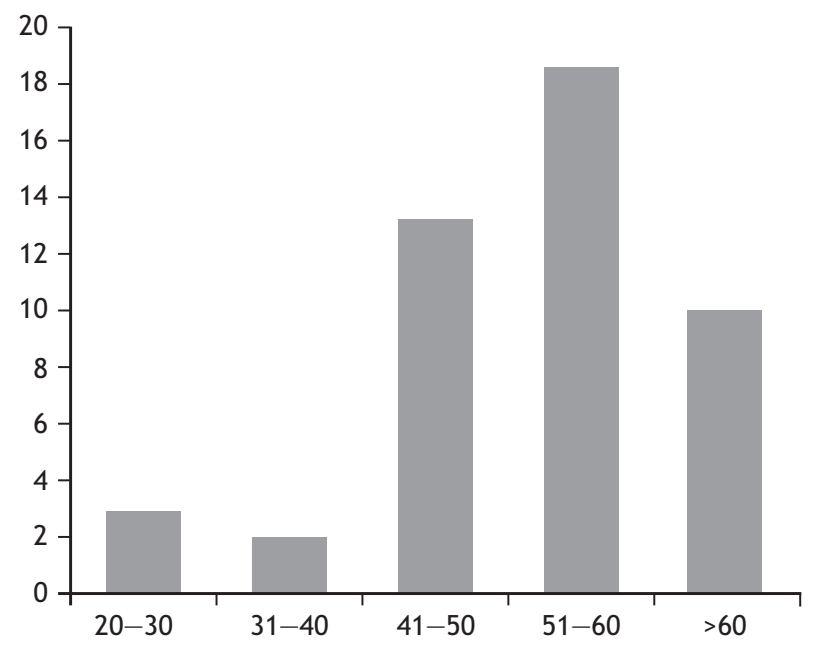

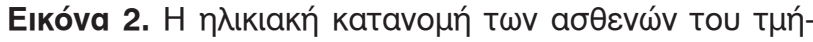

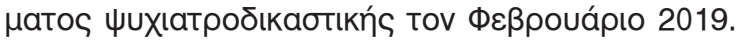

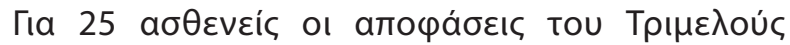

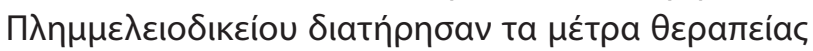

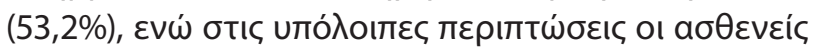

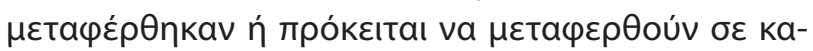

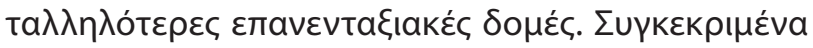

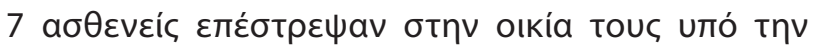

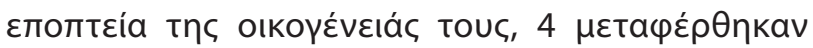

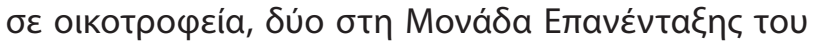

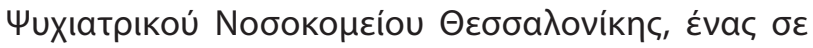

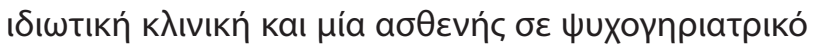

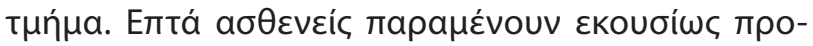

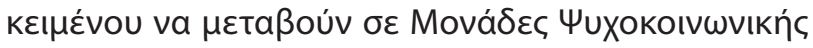
Апоката́бтабпఢ.

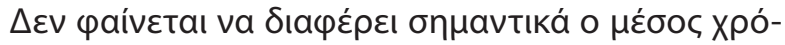

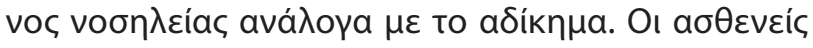

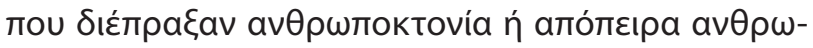

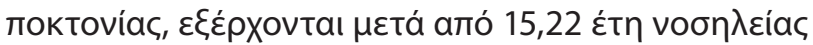

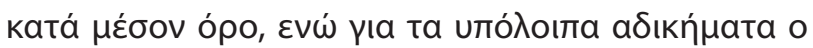

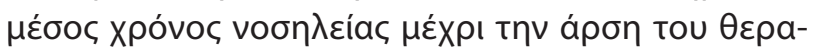

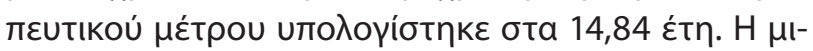

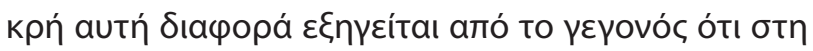

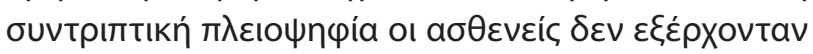

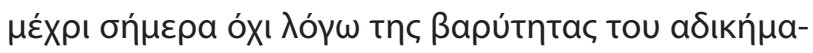

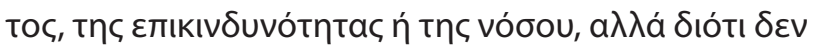

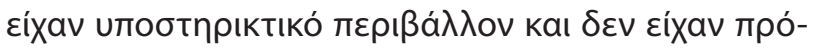

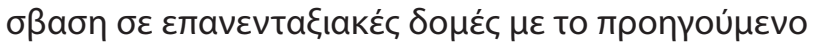

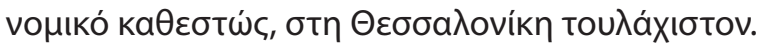

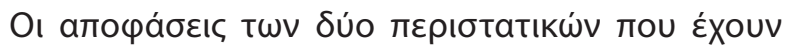

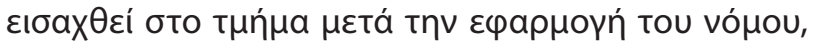

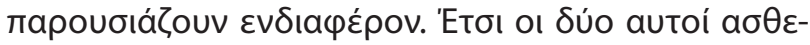




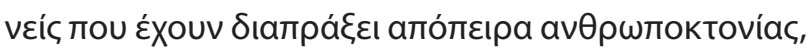

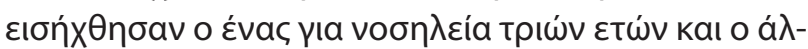

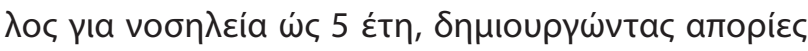

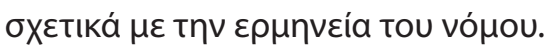

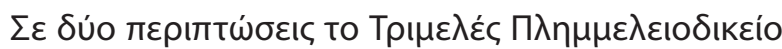

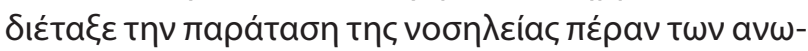

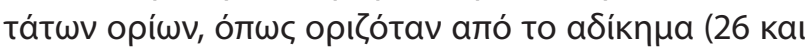

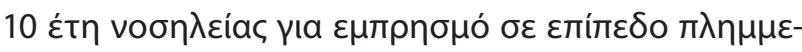

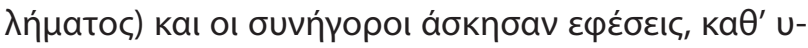

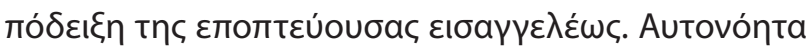

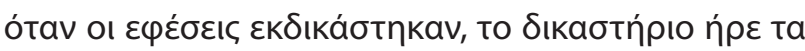

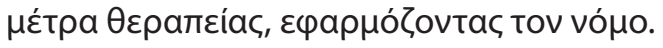

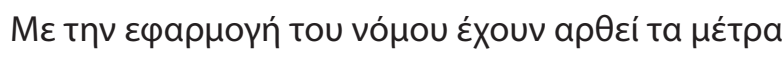

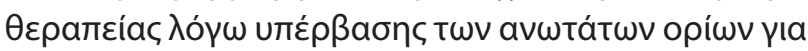

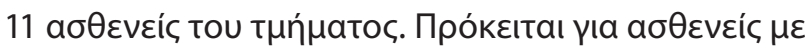

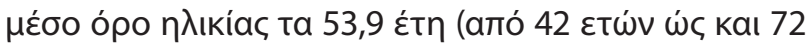

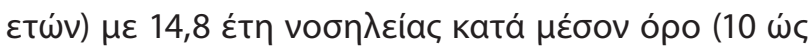

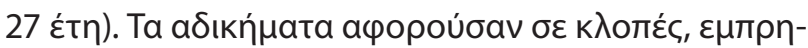

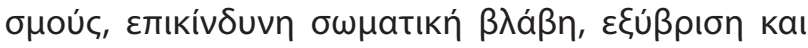

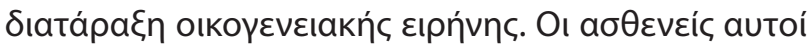

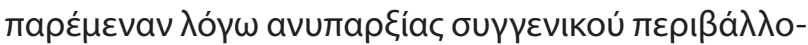

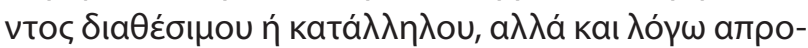

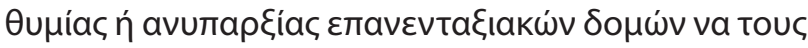

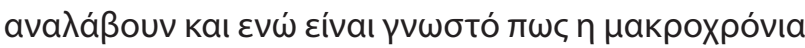

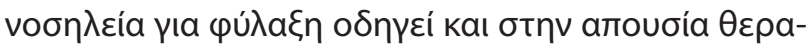

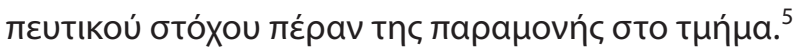

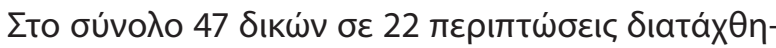

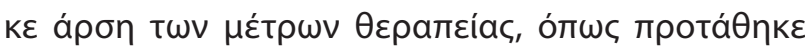

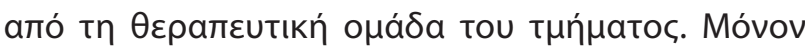

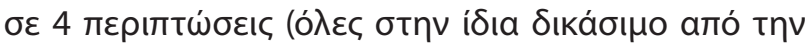

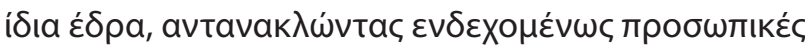

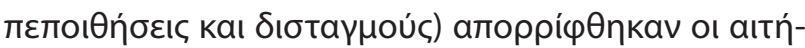

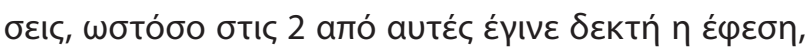

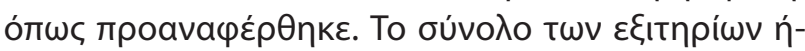

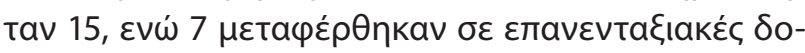

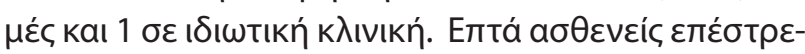

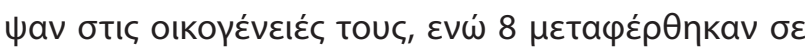

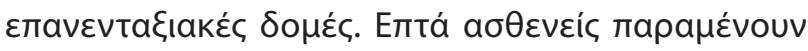

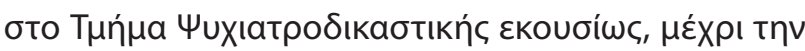

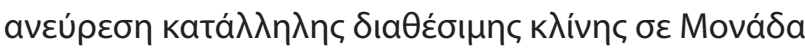

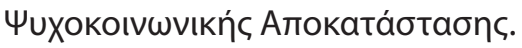

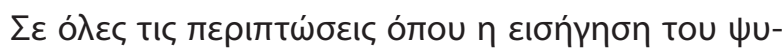

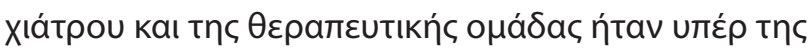

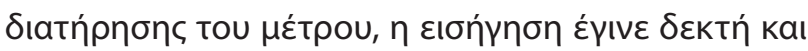

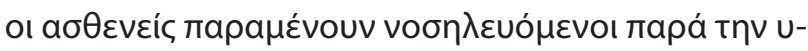

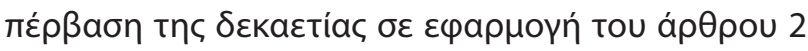

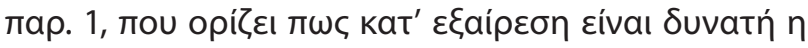

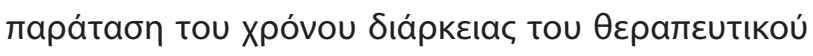

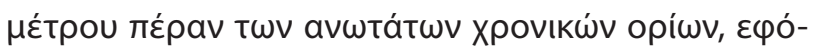

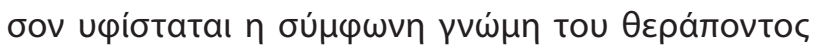

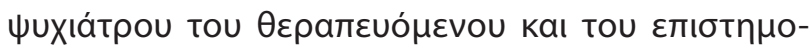

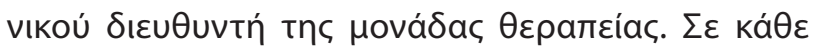

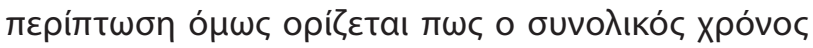

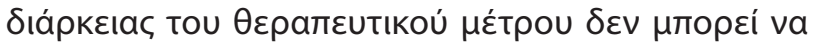

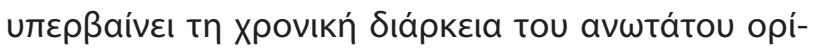

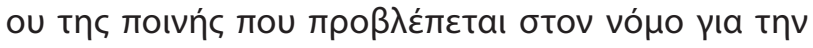

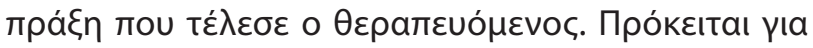

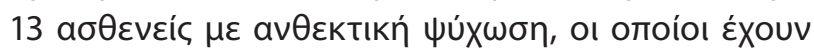

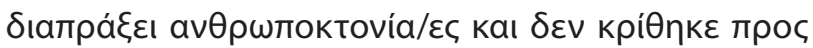

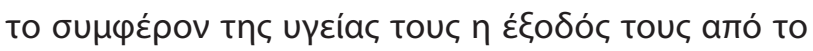

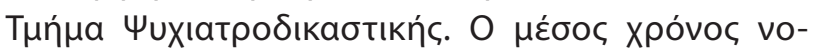

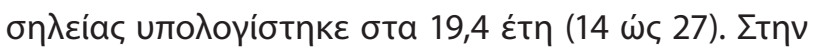
$\pi \lambda \varepsilon ı$

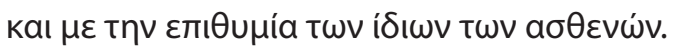

'E६ı a

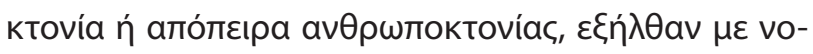

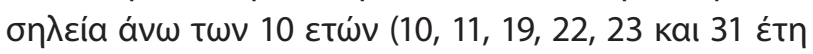

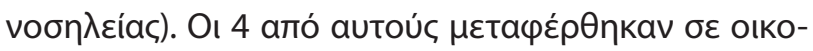

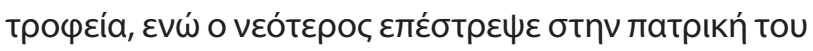

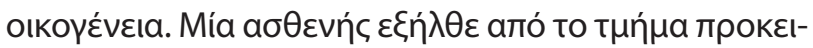

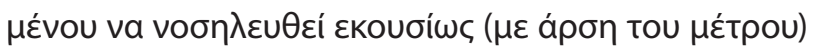

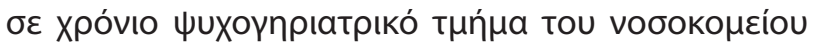

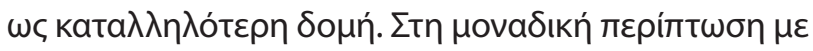

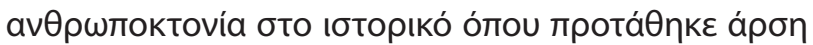

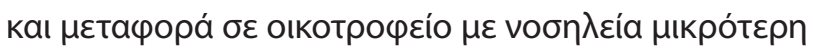

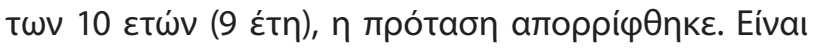

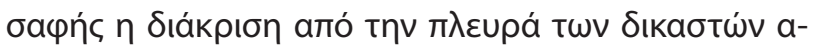

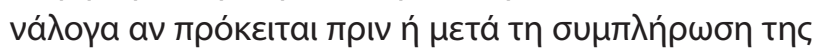

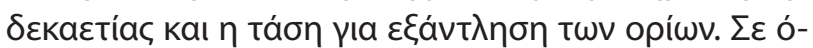

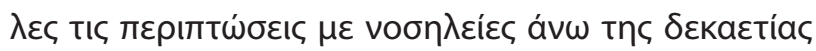

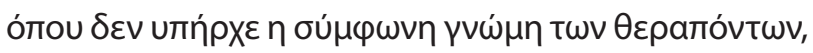

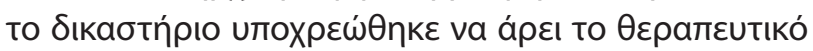

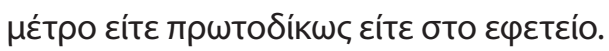

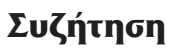

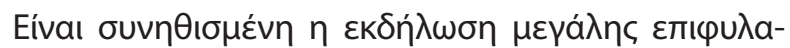

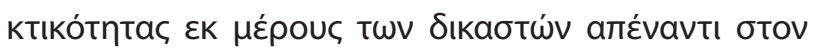

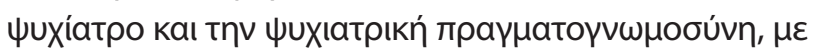

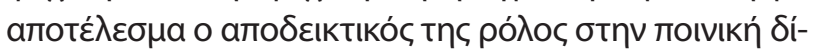

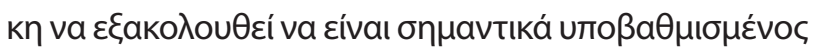

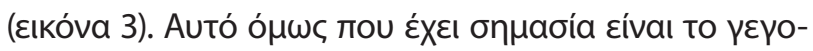

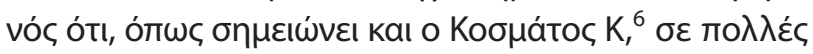

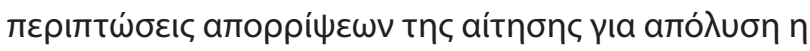

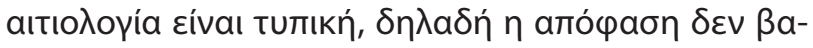




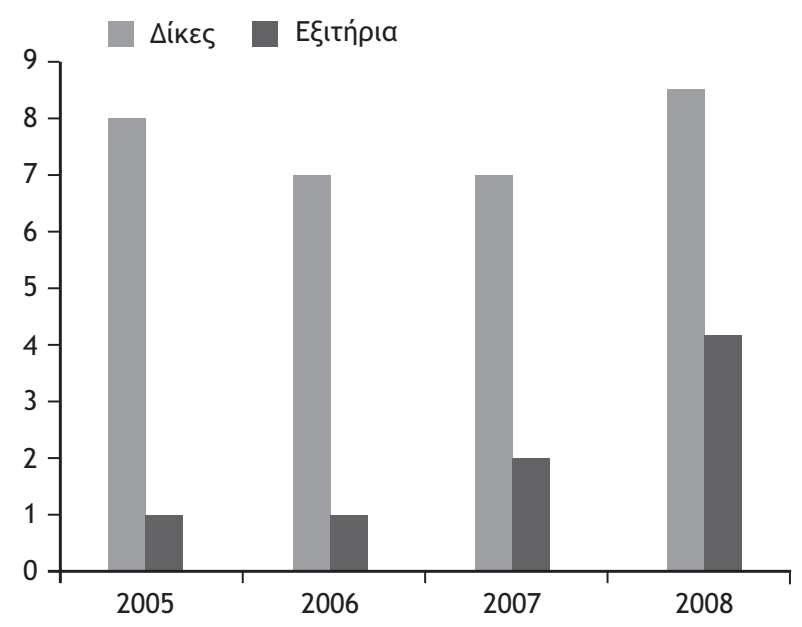

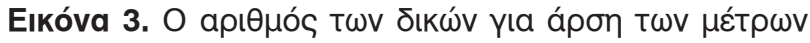

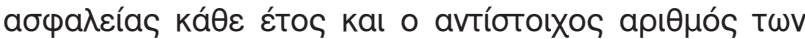

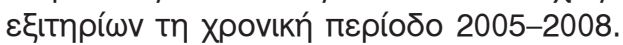

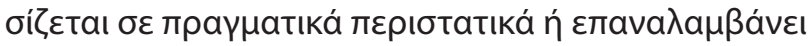

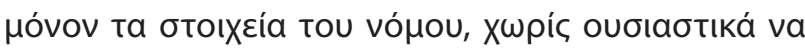

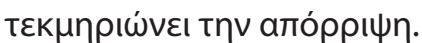

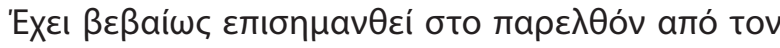

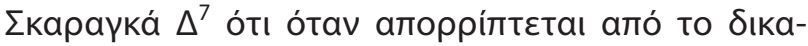

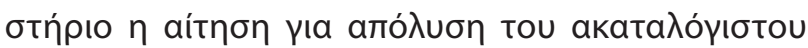

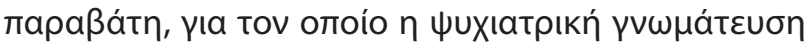

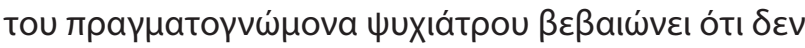

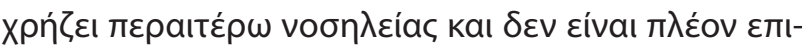

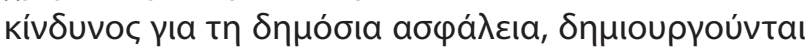

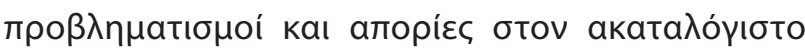

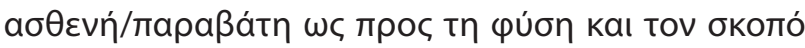

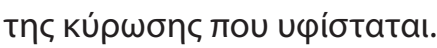

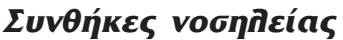

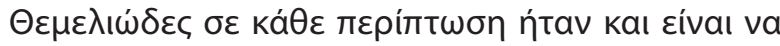

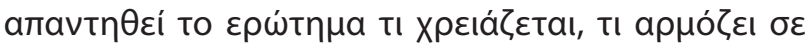

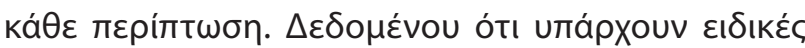

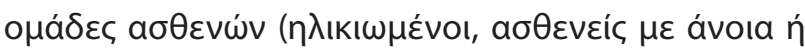

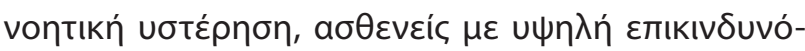

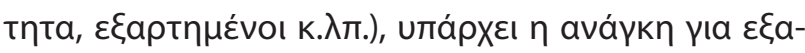

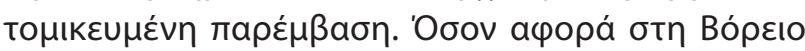

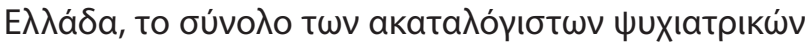

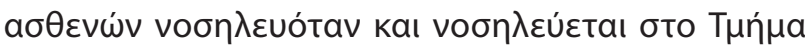

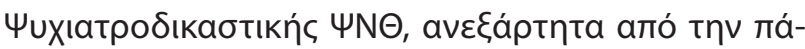

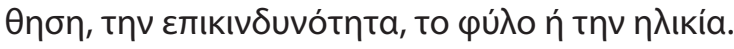

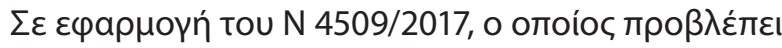

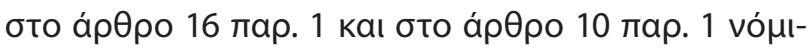

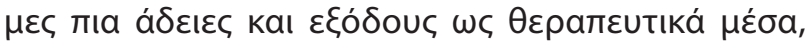

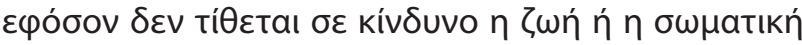

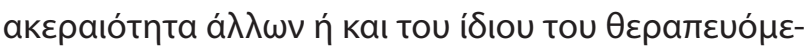

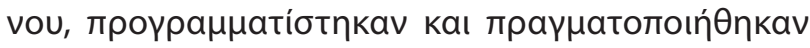

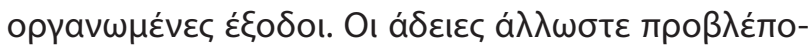

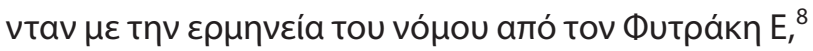

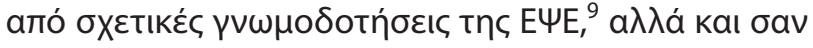

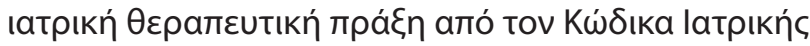

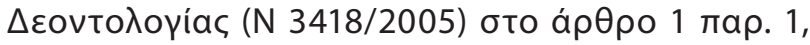

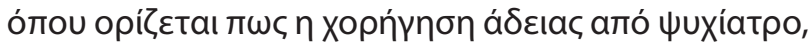

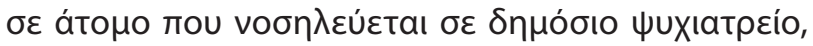

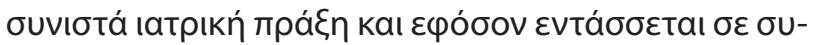

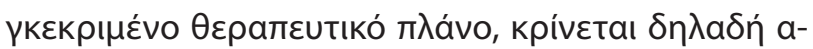

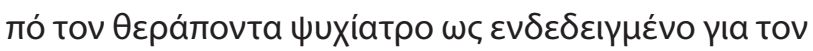

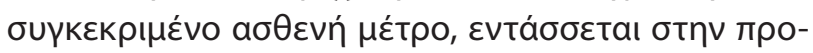

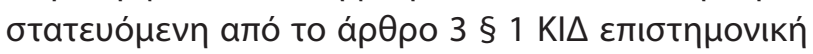

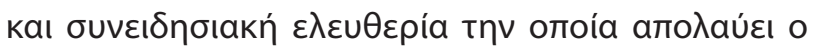

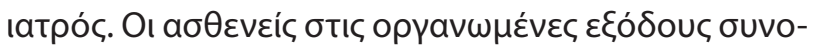

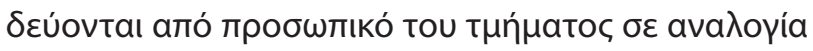
пврі́тои 1:3.

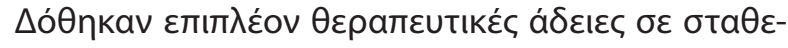

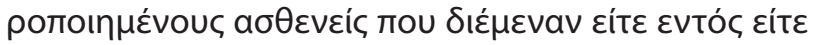

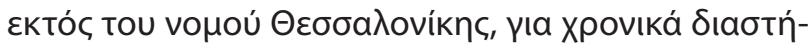

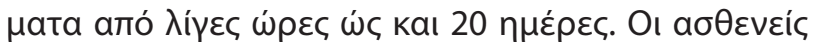

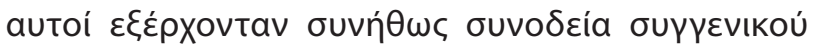

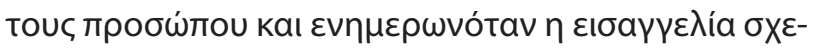

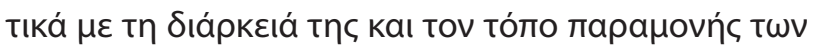

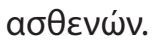

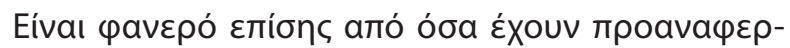

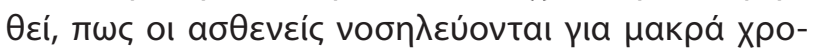

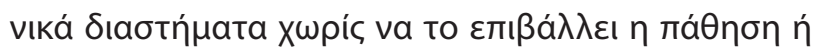

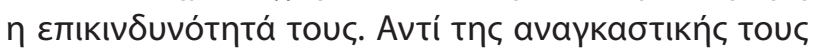

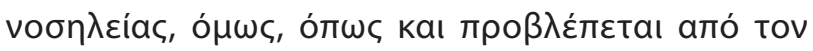

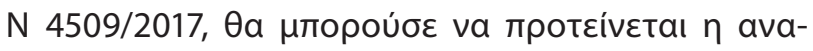

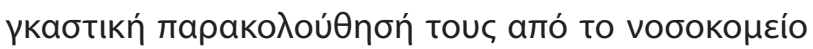

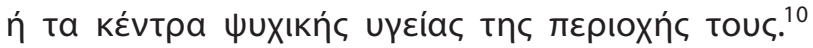

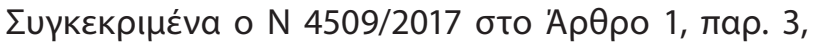

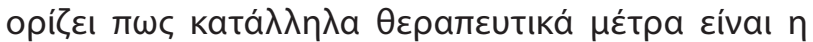

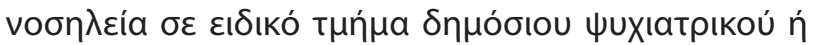

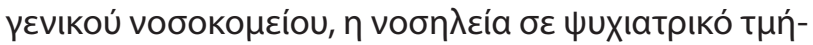

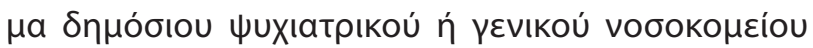

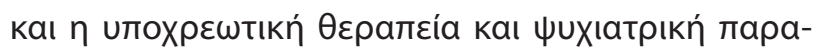

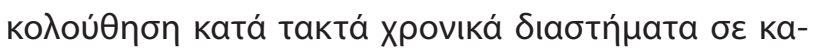

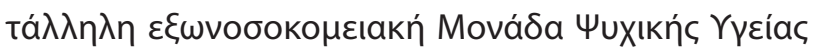

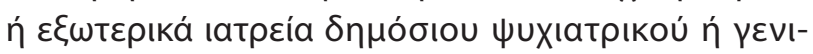

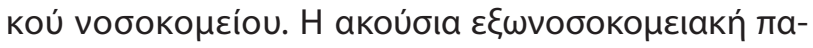

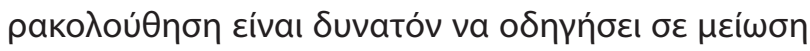

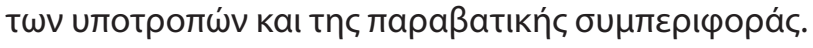

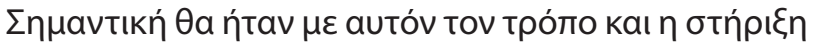




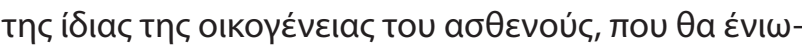

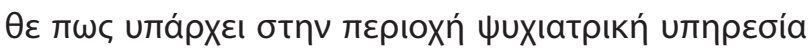

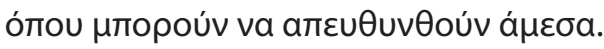

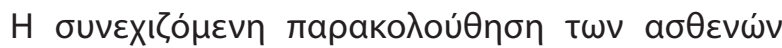

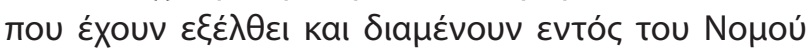

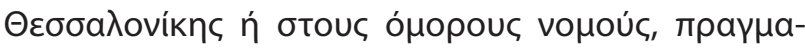

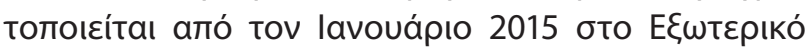

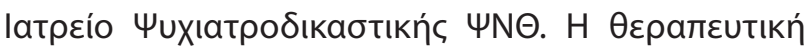

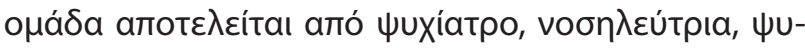

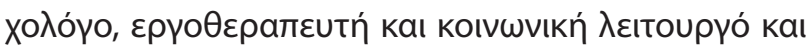

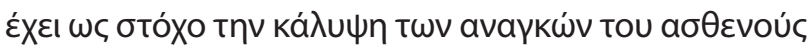

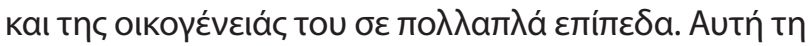

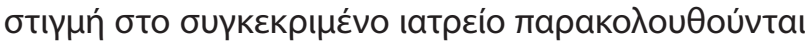

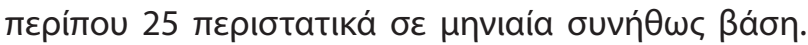

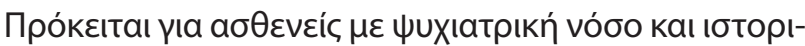

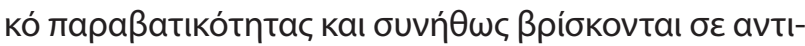

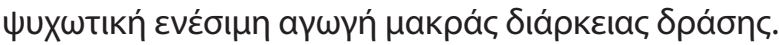

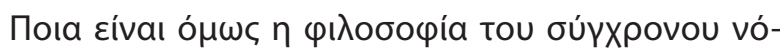

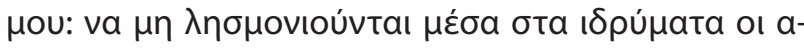

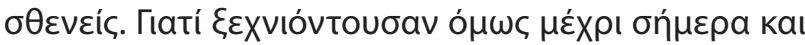

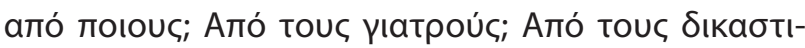

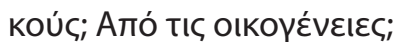

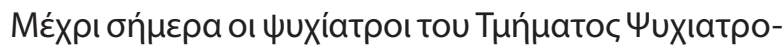

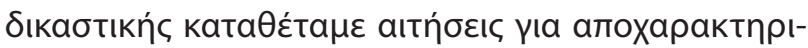

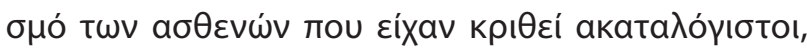

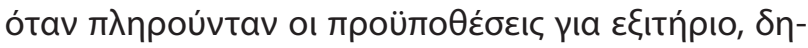

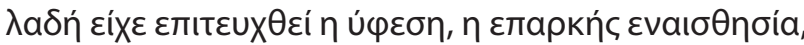

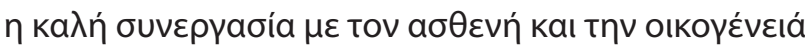

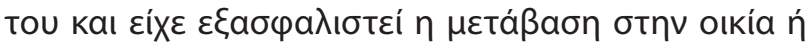

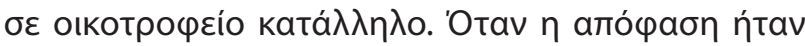

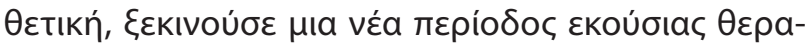

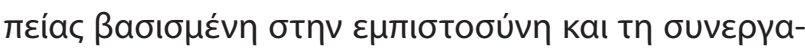

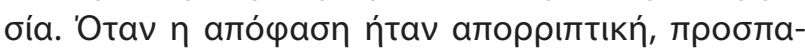

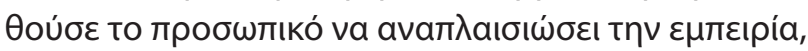

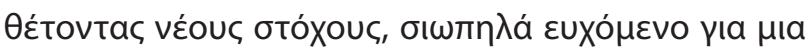

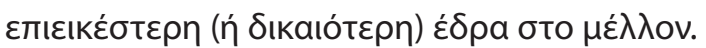

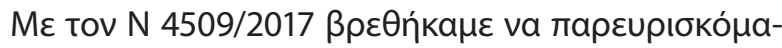

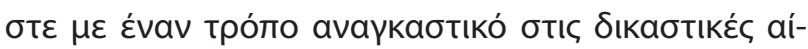

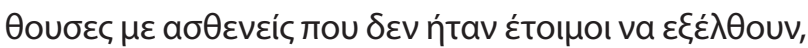

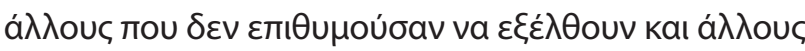

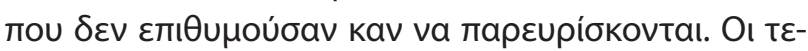

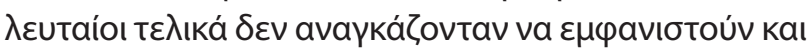

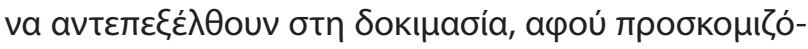

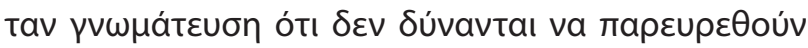

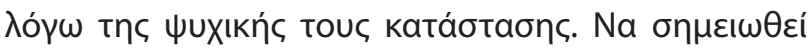

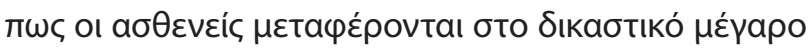

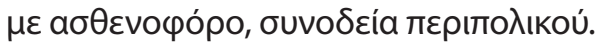

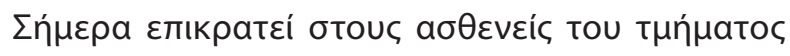

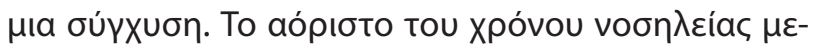

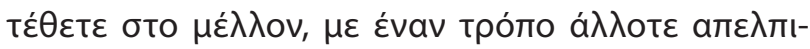

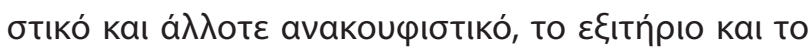

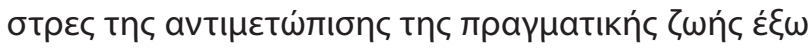

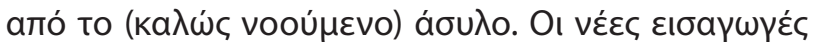

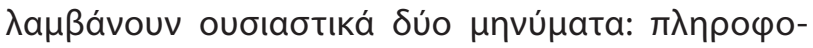

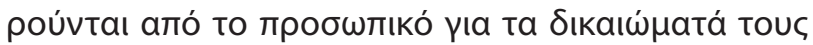

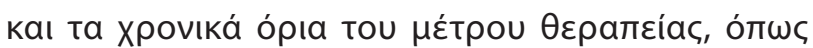

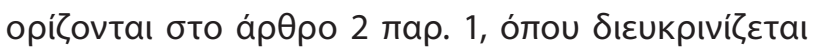

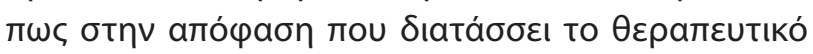

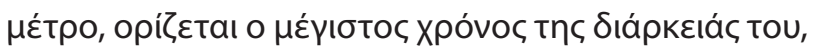

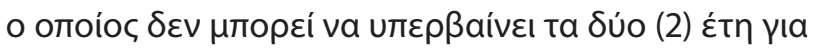

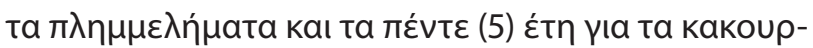

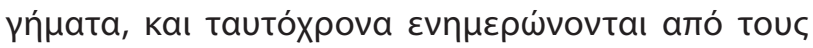

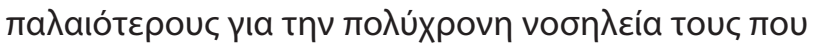

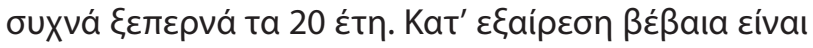

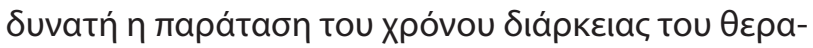

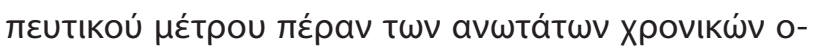

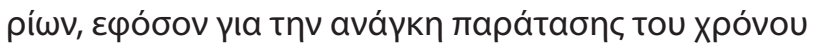

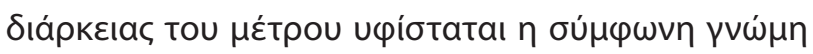

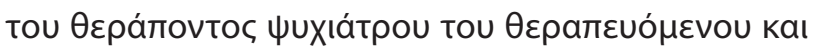

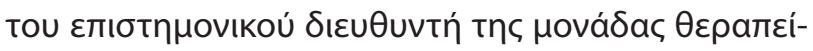

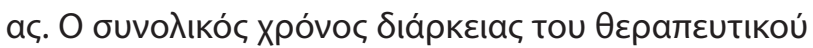

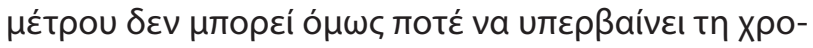

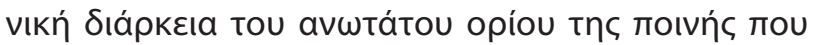

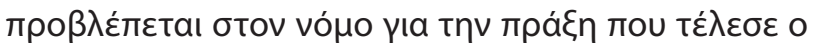

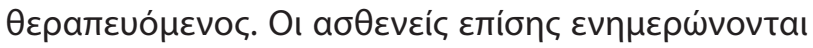

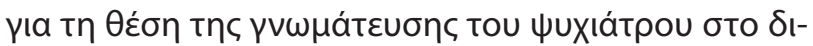

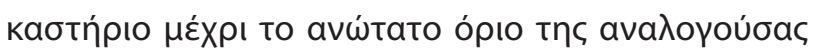

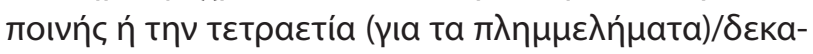

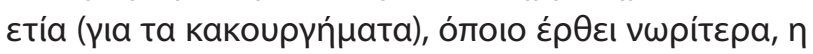

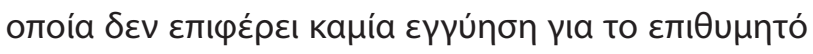

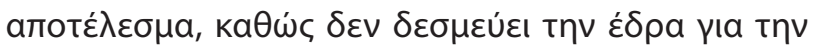

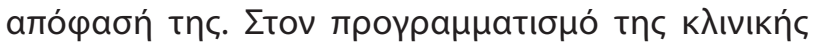

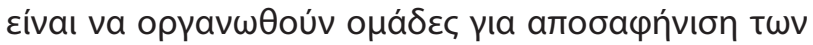

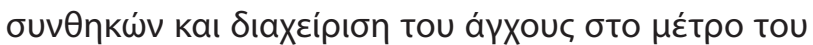

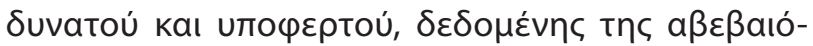

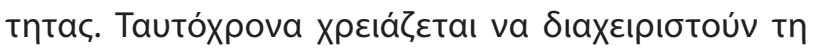

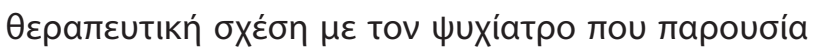

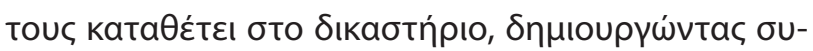

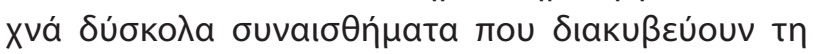

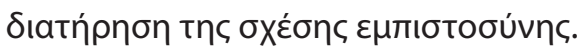

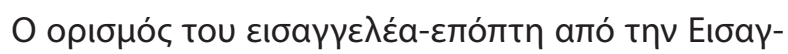

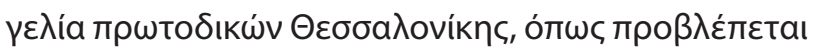

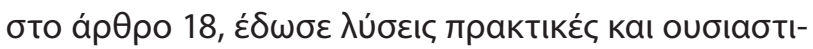

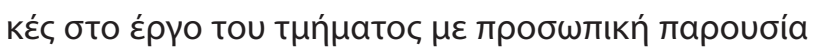




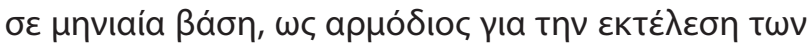

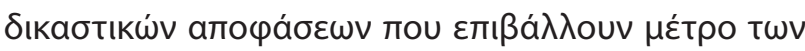

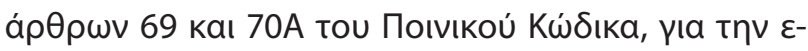

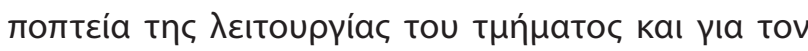

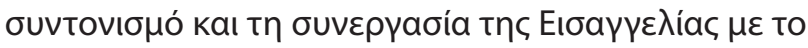

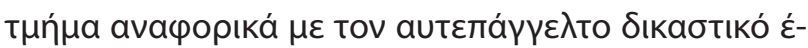

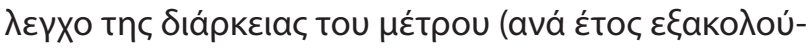

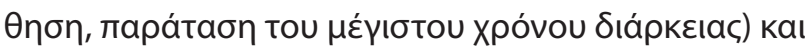

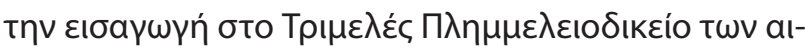

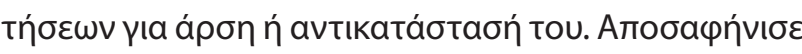

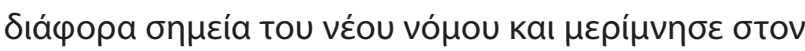

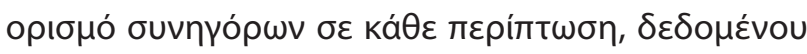

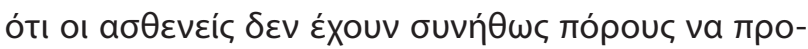

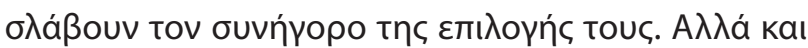

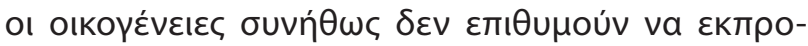

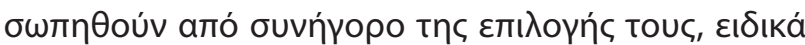

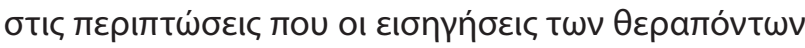

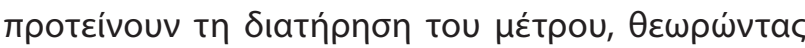

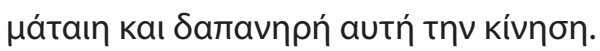

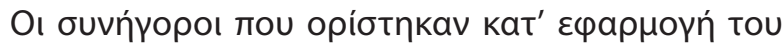

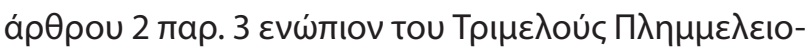

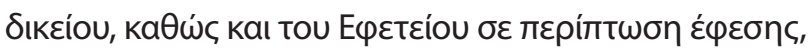

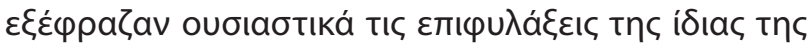

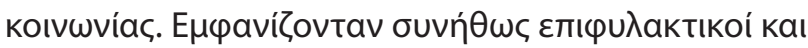

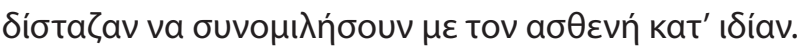

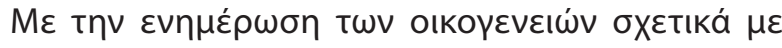

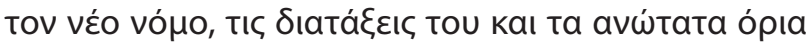

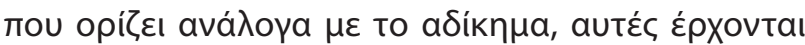

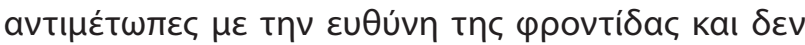

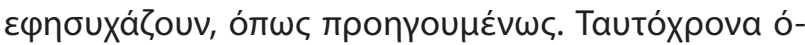

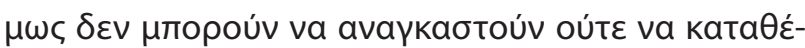

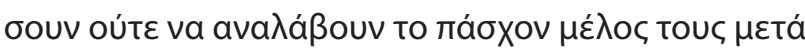

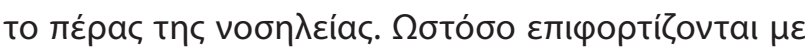

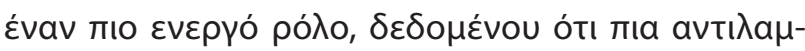

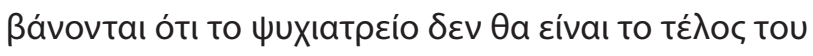

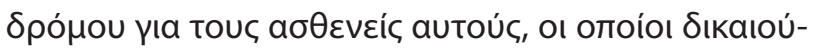

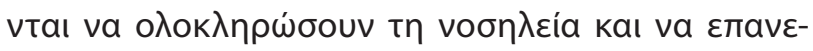

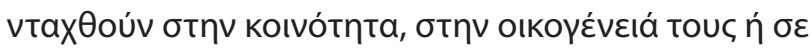

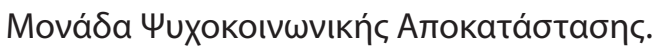

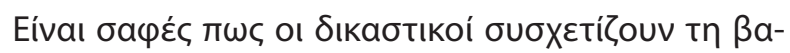

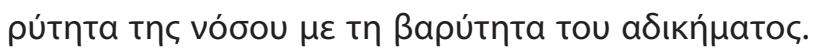

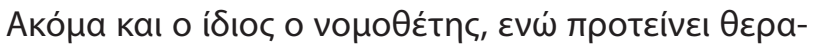

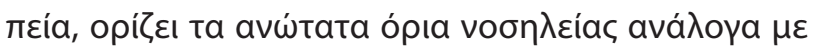

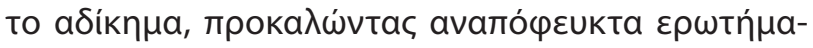

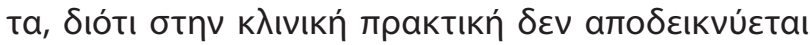

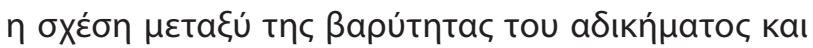

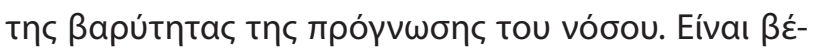

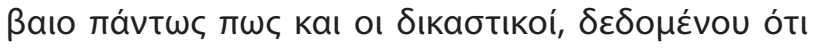

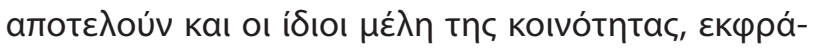

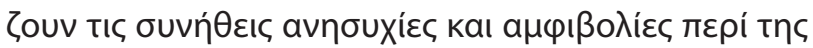

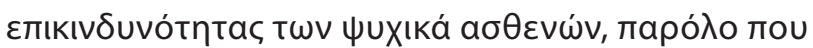

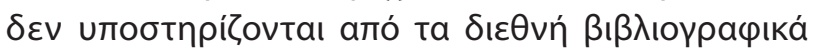

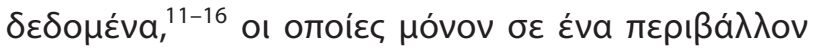
a

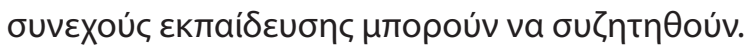

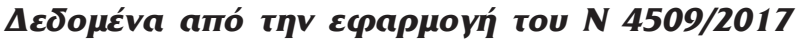

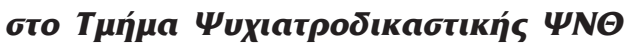

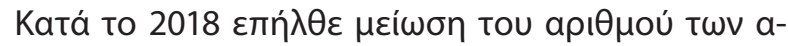

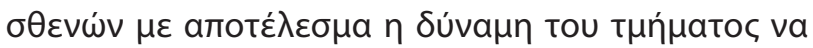

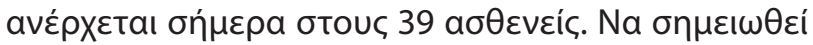

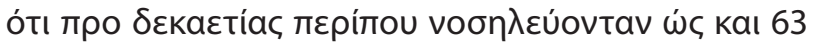

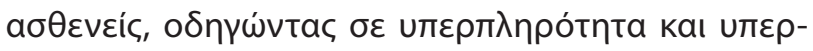

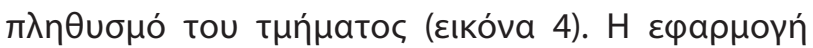

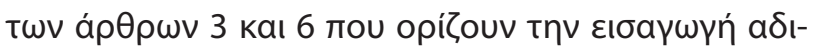

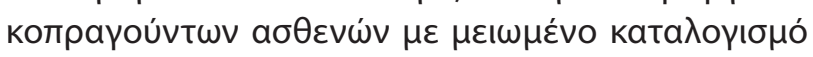

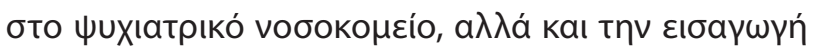

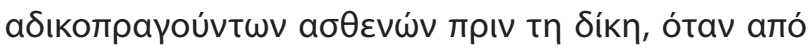

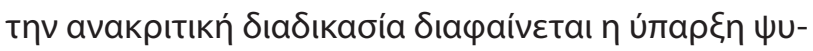

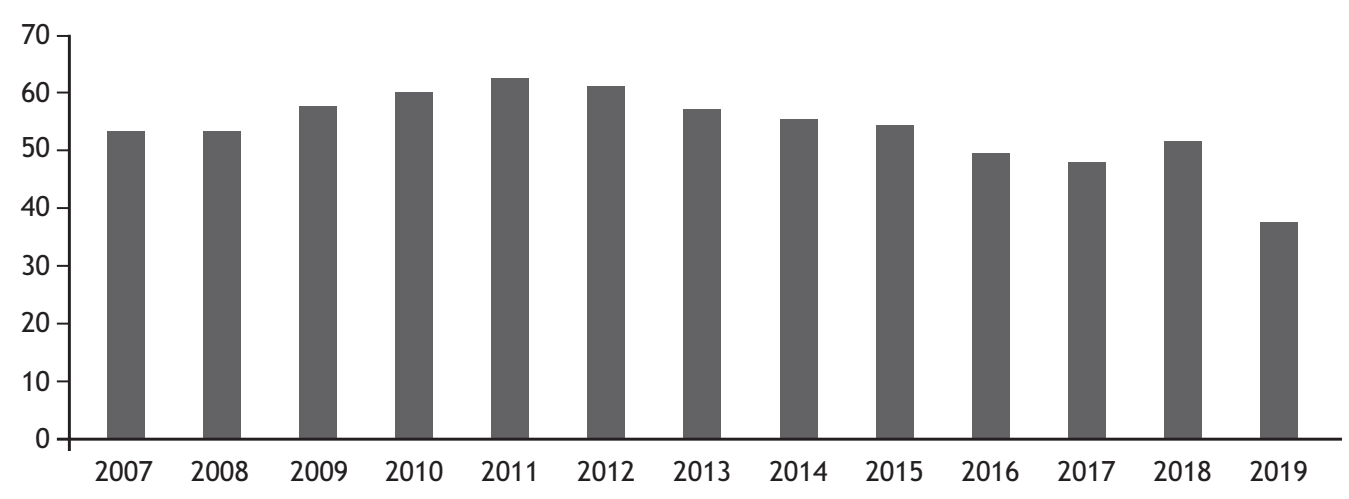

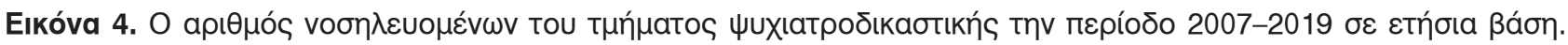




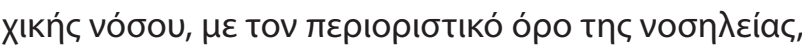

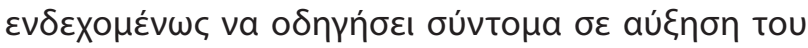

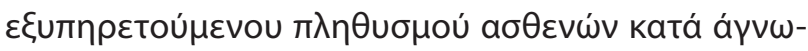

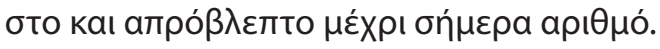

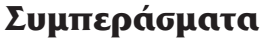

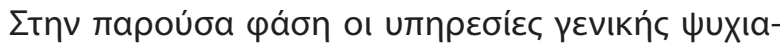

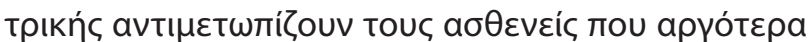

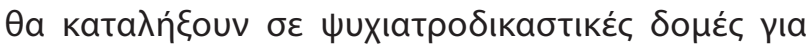

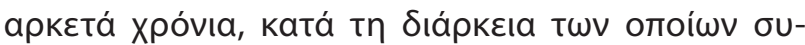

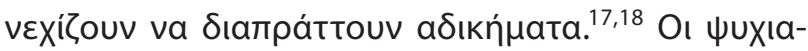

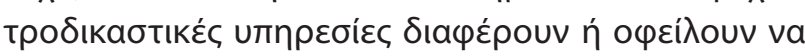

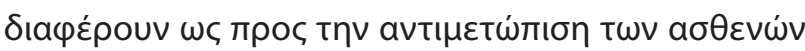

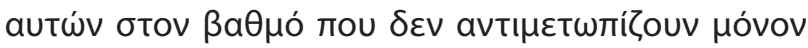

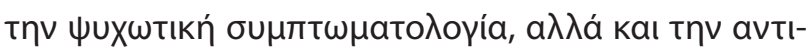

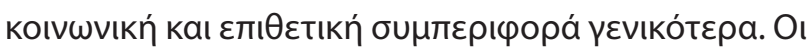

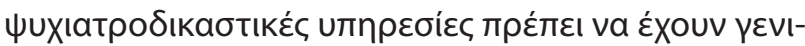

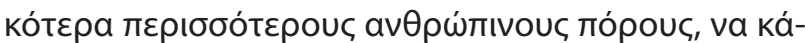

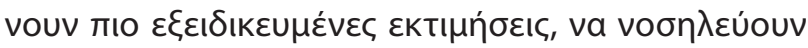

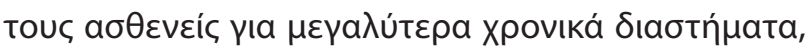

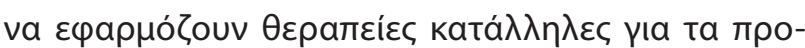

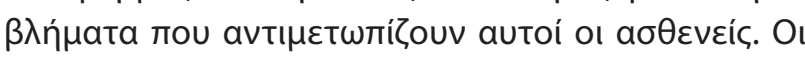

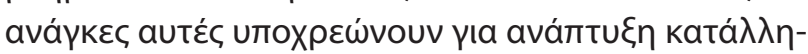

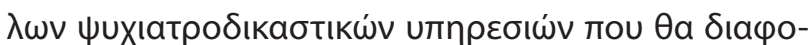

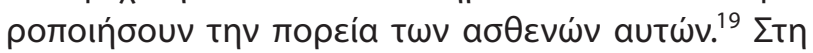

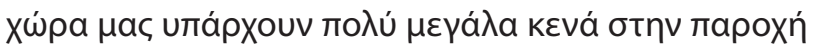

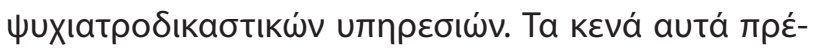

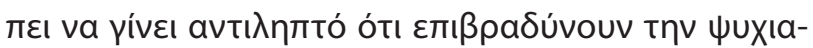

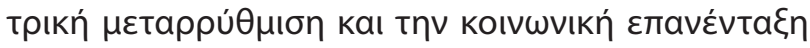

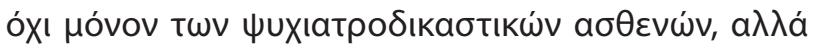

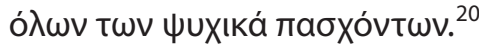

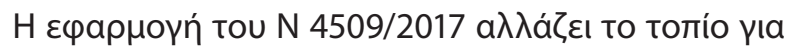

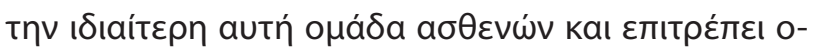

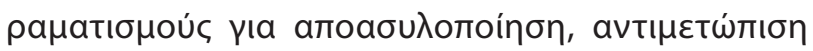

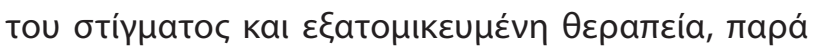

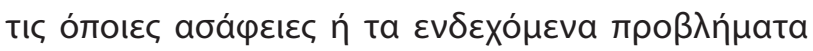

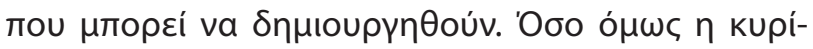

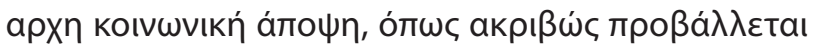

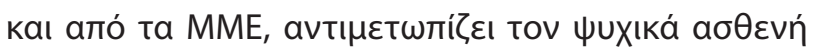

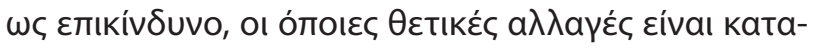

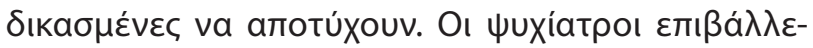

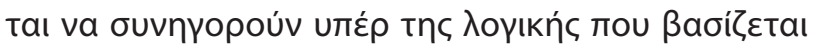

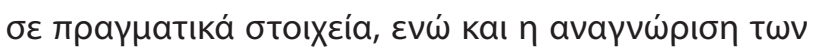

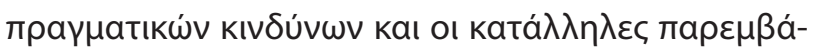

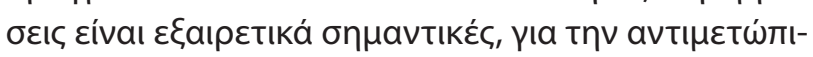

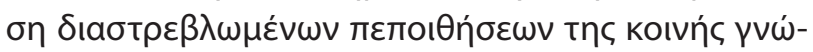

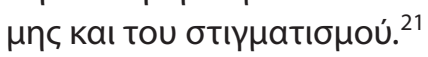

\title{
The implementation of the law 4509/2017 in the Forensic Department of the Psychiatric Hospital of Thessaloniki
}

\author{
M. Markopoulou, ${ }^{1}$ K. Iliou, ${ }^{1}$ V. Karakasi, ${ }^{2}$ Ath. Douzenis ${ }^{3}$ \\ ${ }^{1}$ Forensic Department, General Hospital of Thessaloniki Georgios Papanikolaou- \\ Psychiatric Hospital of Thessaloniki, Organic Unit Psychiatric Hospital of Thessaloniki, \\ ${ }^{2} 3 \mathrm{rd}$ Department of Psychiatry, Aristotle University of Thessaloniki, Medical School, AXEPA Hospital, Thessaloniki, \\ ${ }^{3}$ 2nd Department of Psychiatry, University of Athens, Medical School, "Attikon" Hospital, Athens, Greece
}

Psychiatriki 2020, 31:13-22

According to the Explanatory Memorandum of the law 4509/2017, a significant change is being made to the current institutional framework of Articles 69 and 70 of the ECHR concerning the penal treatment of mentally ill offenders, in order to ensure a high level of treatment for perpetrators with mental or intellectual disorder. In application of the law, it was examined in court the abolishment, maintenance or replacement of the treatment measure of 47 patients from the Department 
of Forensic Psychiatry in Thessaloniki. The results were compared with the previous status quo. In 22 cases, abolishment was ordered for the patients to be discharged and return to their parent's home or to Psychosocial Rehabilitation Units. In 11 of them, their stay was in direct violation of the law due to exceeding the cap as defined by the severity of the offense. However, 7 patients remain voluntarily in the Department, as no beds are available in reintegration structures. 13 patients who have committed homicide remain with the psychiatrist's agreement beyond the overrunning the ten years of treatment due to the severity of the disease or the risk for violent behavior. The Public Prosecutor has provided solutions in many cases and has appointed lawyers for all patients as prescribed by law. However, the judiciary remains cautious and the trend towards exhaustion of the limits is clear, despite the fact that their fears are not confirmed by international bibliographic data. The application of N 4509/2017 attempts to change the landscape for this particular group of patients and allows visions for de-institutionalization, elimination of the stigma and personalized treatment, despite any ambiguities or potential problems that may arise. However, as the predominant social viewpoint, shown by the media, treats the mentally ill as dangerous, any positive changes are doomed to fail. In addition, it is necessary to develop psychiatric services for the treatment of patients which will not only treat psychotic symptoms but also antisocial and aggressive behavior in general.

Key words: Law, prosecutor, mentally ill offenders, trial, forensic psychiatry.

\section{BıßAtoypacpía}

1. Mental health and human rights. Report of the United Nations High Commissioner for Human Rights, 31.01.2017, article 32

2. Mental Health and prisons. The challenge, World Health Organization, International Committee of Red Cross, cited 1/12/2018, available from https://www.who.int/mental_health/ policy/mh_in_prison.pdf

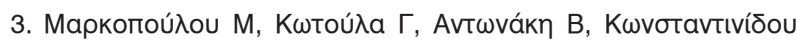

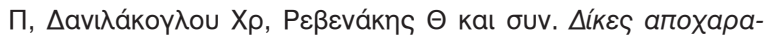

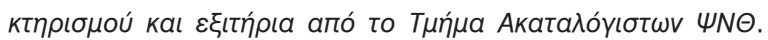

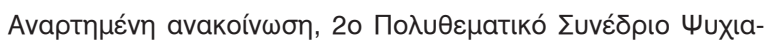

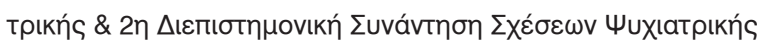

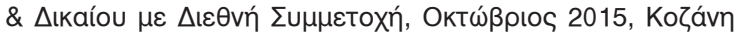

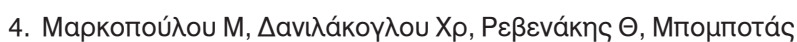

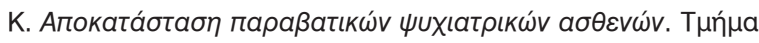

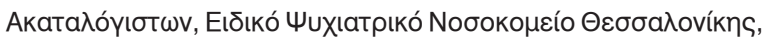

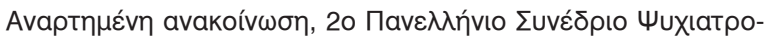

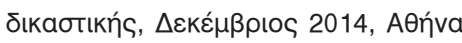

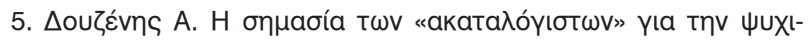

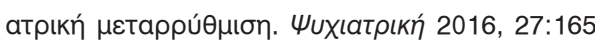

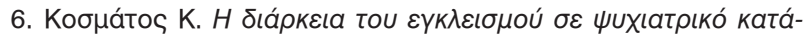

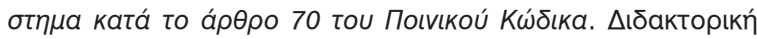

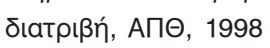

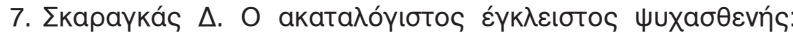

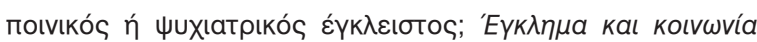
1987, 3:133-134

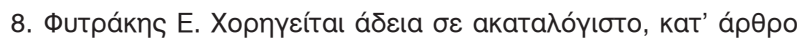

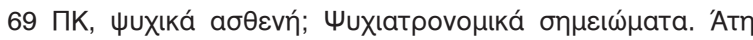
2014, 7:10-12

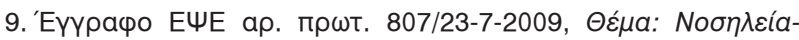

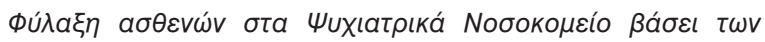

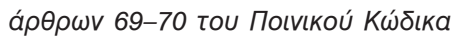

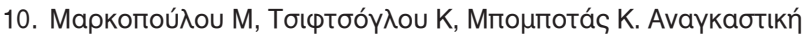

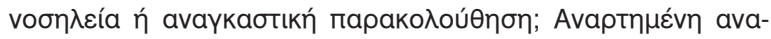

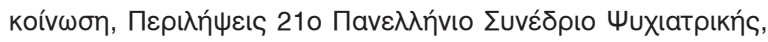
AӨńva 2011

11. Sturup J, Lindqvist $P$. Homicide offenders 32 years later - A Swedish population based study on recidivism. Crim Behav Mental Health 2014, 24:5-17, doi: 10.1002/cbm.1896

12. Golenkov A, Large M, Nielssen O. A 30-year study of homicide recidivism and schizophrenia, Crim Behav Mental Health 2013, 23:347-355, doi:10.1002/cbm. 1876

13. Nielssen OB, Bourget D, Laajasalo $T$, Liem M, Labelle A, Hakkanen-Nyholm $\mathrm{H}$ et al. Homicide of strangers by people with a psychotic illness. Schizophr Bull 2011, 37:572-579, doi: 10.1093/schbul/sbp112

14. Nielssen OB, Yee NL, Millard MM, Large MM. Comparison of first-episode and previously treated persons with psychosis found NGMI for a violent offense. Psychiatr Serv 2011, 62:759-764, doi: 10.1093/schbul/sbn144

15. Schanda H, Knecht G, Schreinzer D, Stompe Th, OrtweinSwoboda G, Waldhoer Th. Homicide and major mental disorders: a 25-year study. Acta Psychiatr Scand 2004, 110:98-107, doi: 10.1111/j.1600-0047.2004.00305.x

16. Simpson Al, McKenna B, Moskowitz A, Skipworth J, BarryWalsh J. Homicide and mental illness in New Zealand, 1970-2000. Br J Psychiatry 2004, 185:394-8, doi: 10.1192/ bjp. 185.5 .394 
17. Hodgins S, Muller-Isberner R. Preventing crime by people with schizophrenic disorders: the role of psychiatric services. $\mathrm{Br}$ J Psychiatry 2004, 185:245-250, doi: 10.1192/bjp. 185.3.245

18. Hodgins S, Müller-Isberner R, Allaire J. Attempting to understand the increase in the numbers of forensic beds in Europe. A multi-site study of patients in forensic and general psychiatric services. Int J Forensic Ment Health Serv 2006, 5:173-184, doi: $10.1080 / 14999013.2006 .10471241$

19. Hodgins S. The interface between general and forensic psychiatric services. Eur Psychiatry 2009, Editorial, 24, 354-355, doi: 10.1016/j.eurpsy.2009.07.002

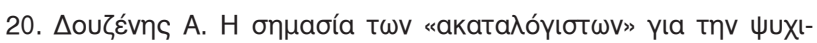

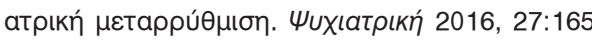

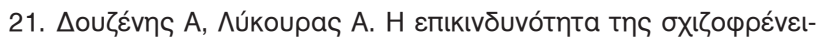

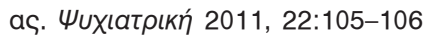

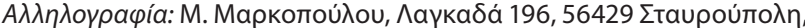

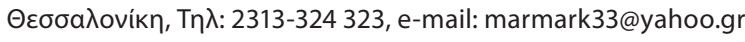




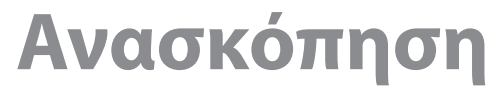 Review
}

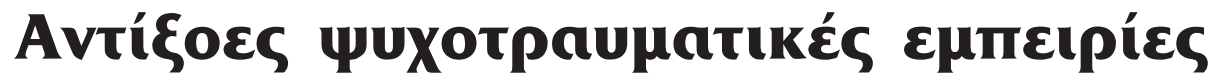

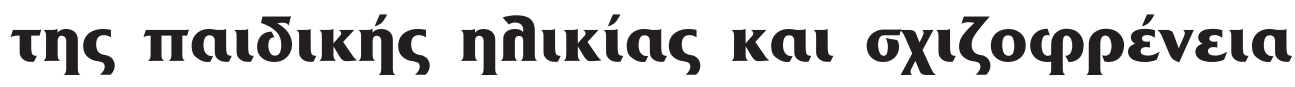

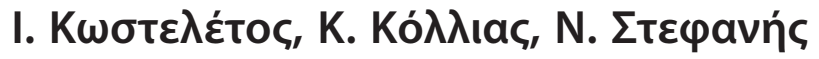

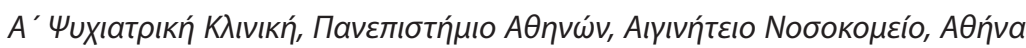

Uuxıатрıкń 2020, 31:23-35

T

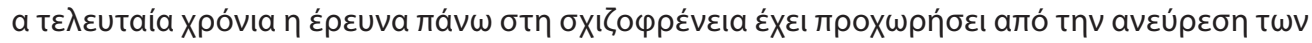

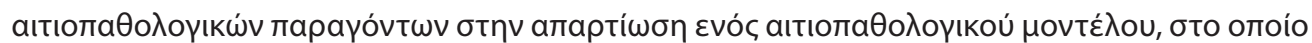

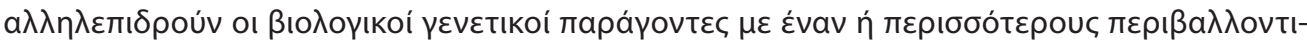

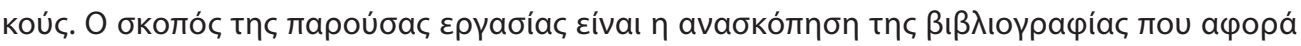

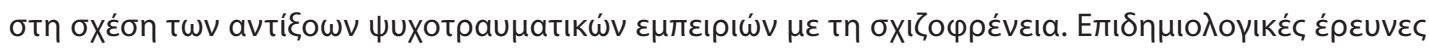

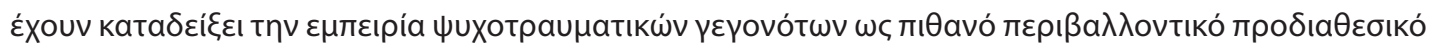

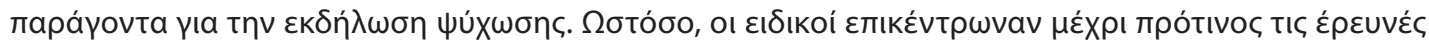

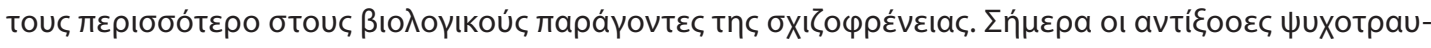

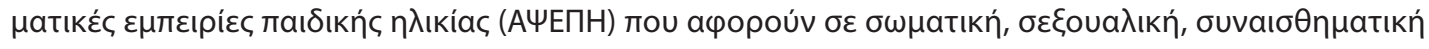

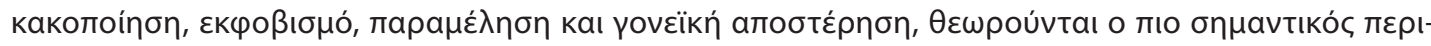

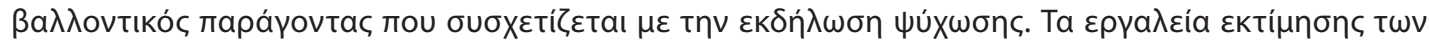

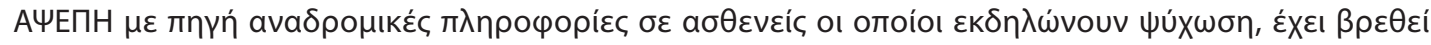

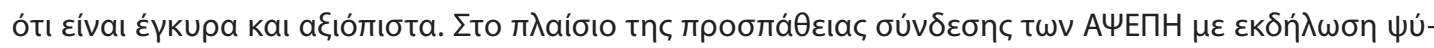

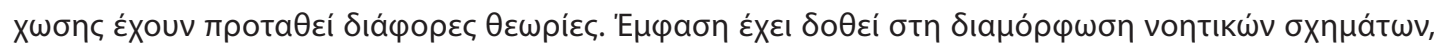

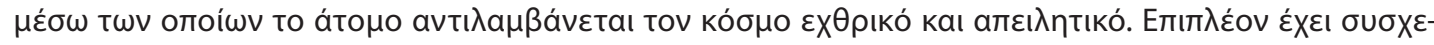

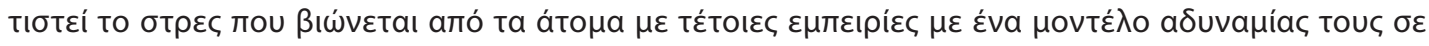

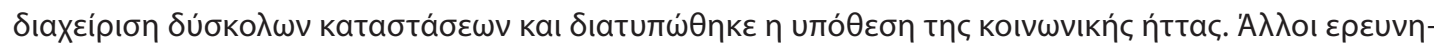

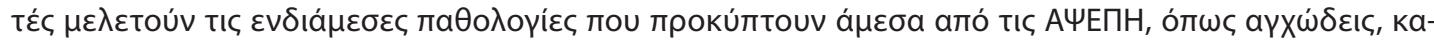
таӨ

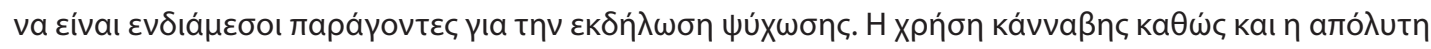

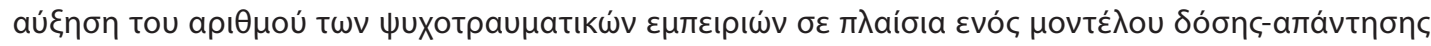

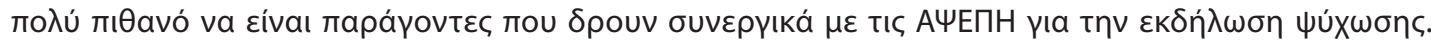

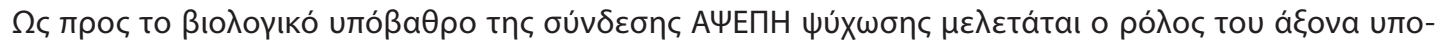




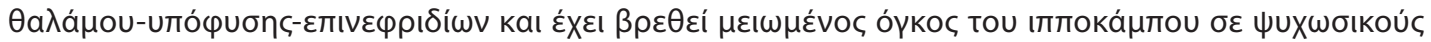

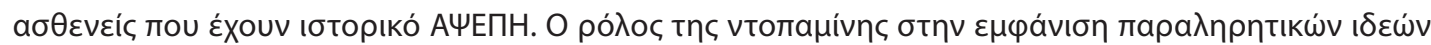

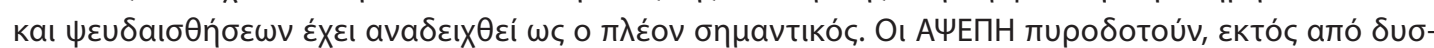

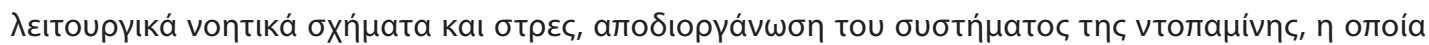

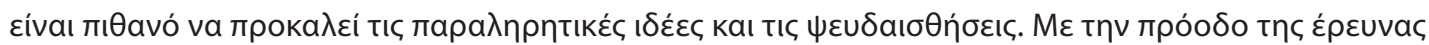

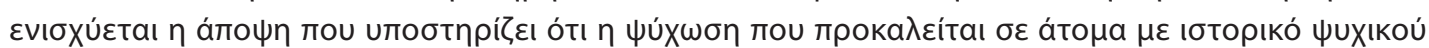

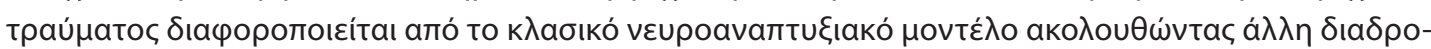

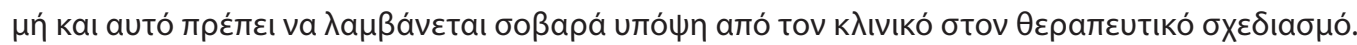

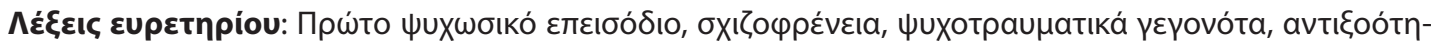
Тદৎ.

\section{Eıaaywyń}

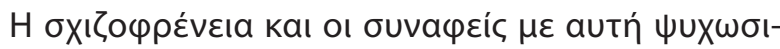

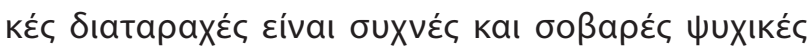

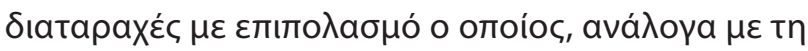

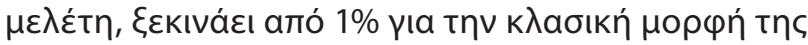

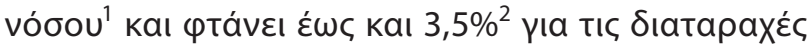

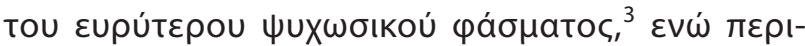

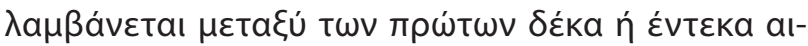

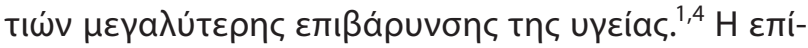

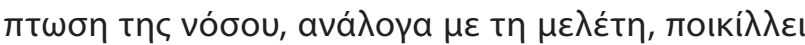

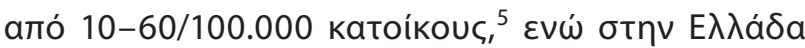

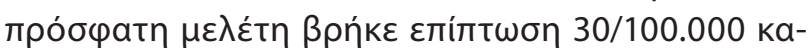

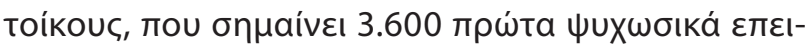

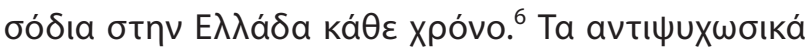

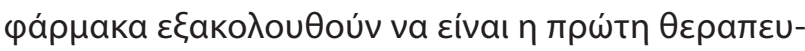

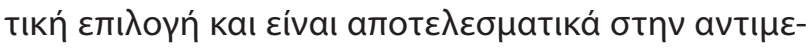

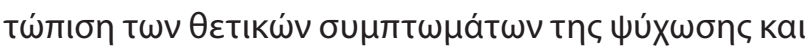

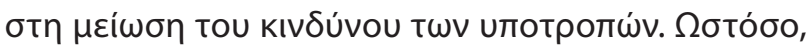

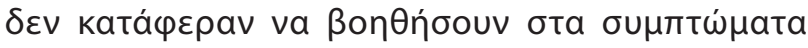

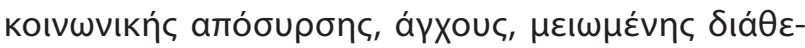

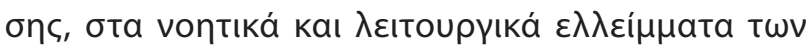

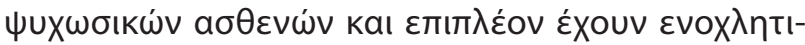

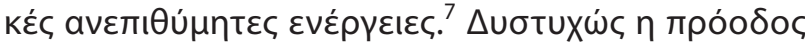

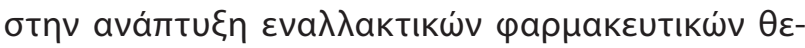

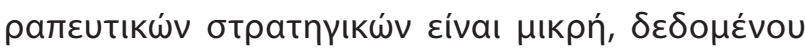

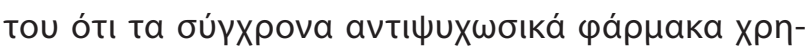

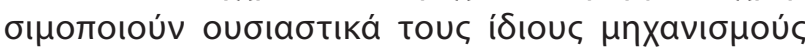

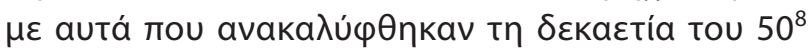

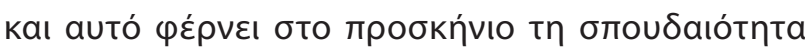

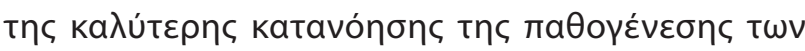

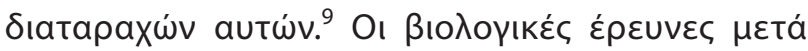

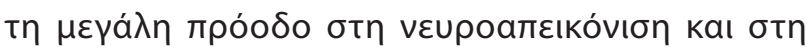

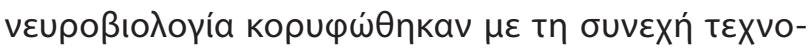

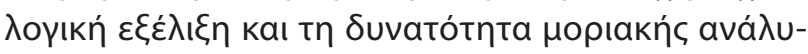

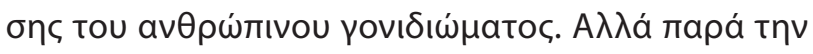

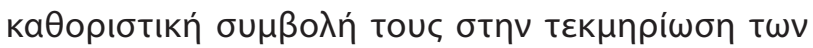

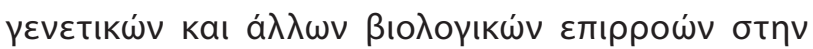

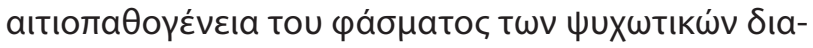

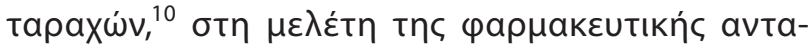

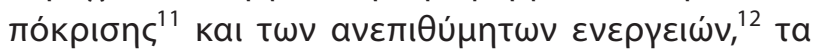

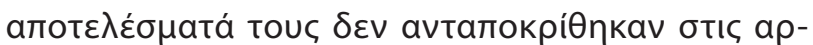

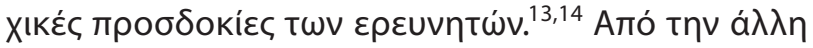

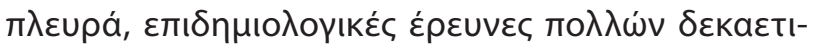

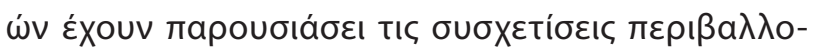

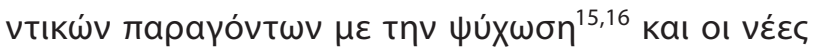

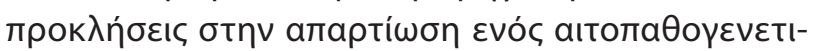

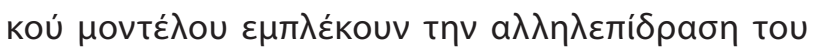

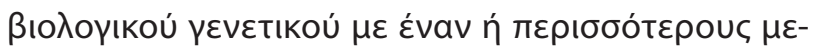

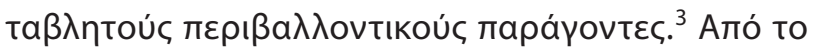

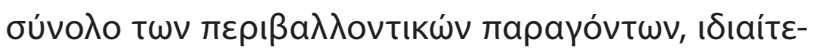

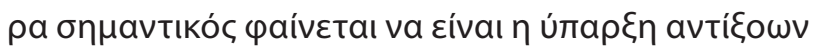

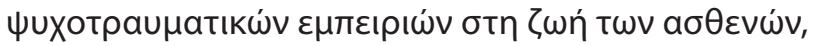

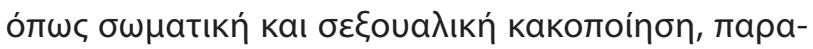

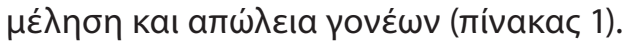

\section{Opı́roí}

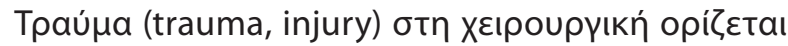

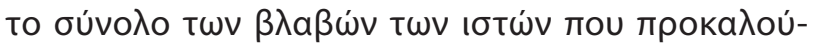

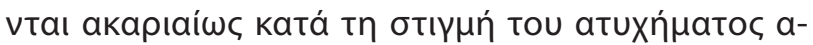

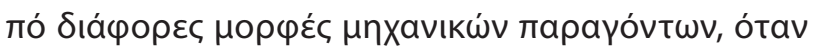

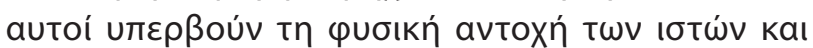

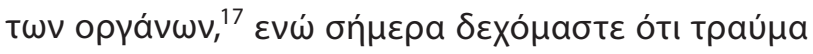

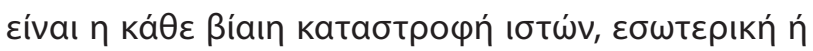

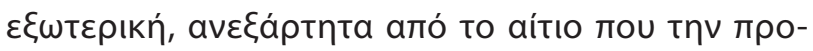

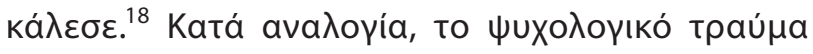

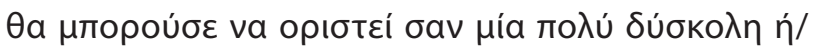

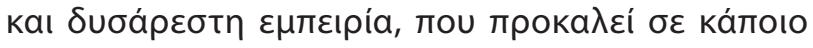

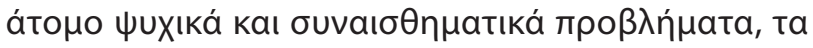




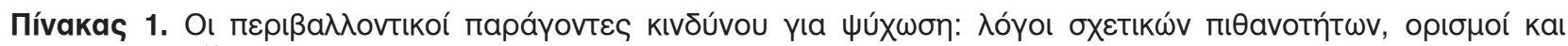
$\beta ı \beta$ ıоррачі́a. ${ }^{16}$

\begin{tabular}{|c|c|c|c|c|}
\hline 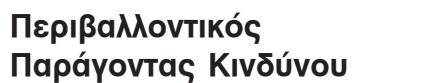 & $\begin{array}{l}\text { Odds } \\
\text { Ratio }\end{array}$ & $\begin{array}{l}\text { Log odd } \\
\text { ratio }\end{array}$ & Avaфоре́ৎ & Opıбноí \\
\hline 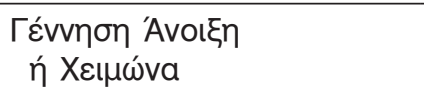 & 1,07 & 0,068 & Davis et al 2003 & 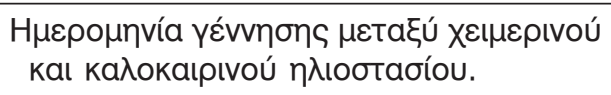 \\
\hline 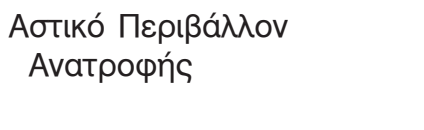 & 1,72 & 0,54 & $\begin{array}{l}\text { Krabbendam \& } \\
\text { van Os } 2005\end{array}$ & 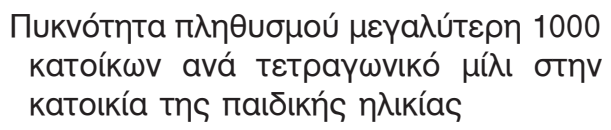 \\
\hline 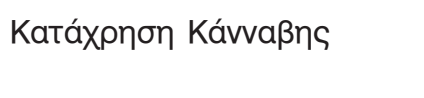 & 1,75 & 0,56 & Kraan et al 2016 & 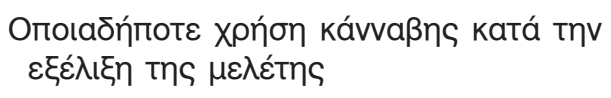 \\
\hline 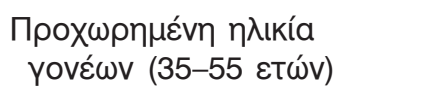 & 1,28 & 0,25 & Torrey et al 2009 & 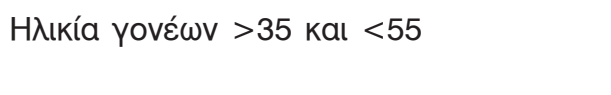 \\
\hline 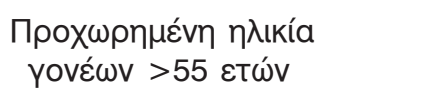 & 2,22 & 0,80 & Torrey et al 2009 & 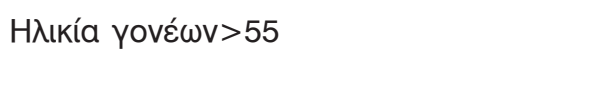 \\
\hline 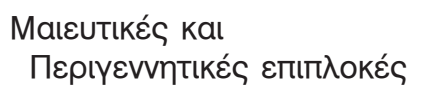 & 2,00 & 0,69 & $\begin{array}{l}\text { Ceddes \& } \\
\text { Lawrie } 1995\end{array}$ & 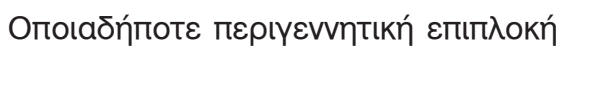 \\
\hline 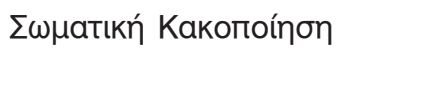 & 2,95 & 1,08 & Varese et al 2012 & 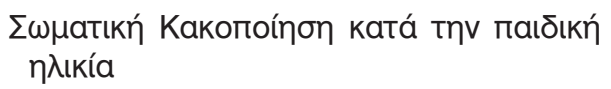 \\
\hline 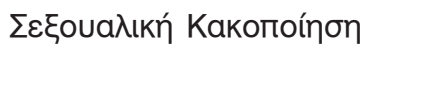 & 2,38 & 0,87 & Varese et al 2012 & 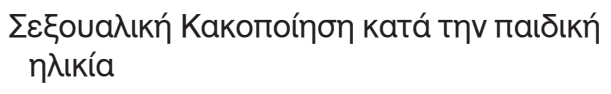 \\
\hline 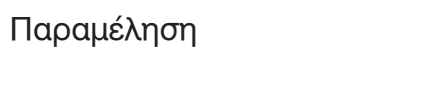 & 2,90 & 1,06 & Varese et al 2012 & 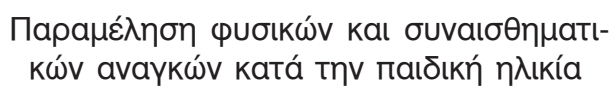 \\
\hline Đávatos yovéa & 1,70 & 0,53 & Varese et al 2012 & 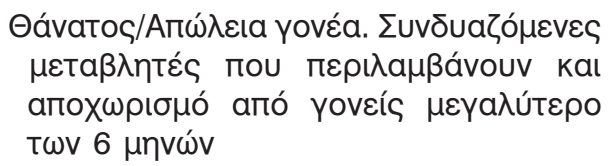 \\
\hline
\end{tabular}

отоі́a кратоúv бuvท́㇒ $\theta \omega \varsigma$ по

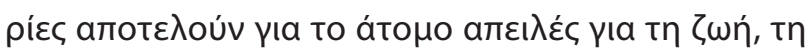

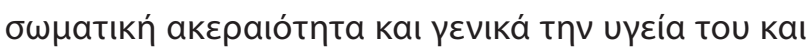

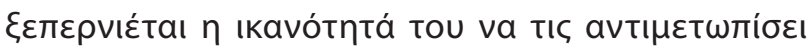

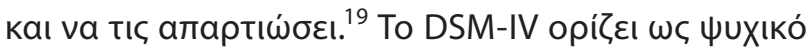

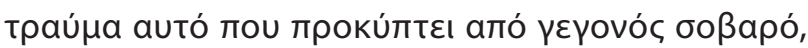

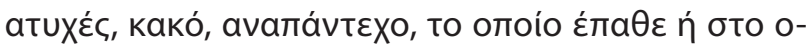

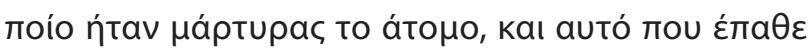

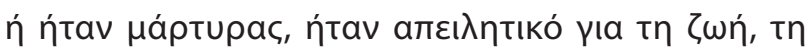

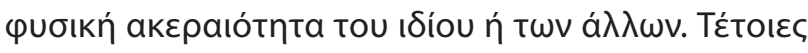

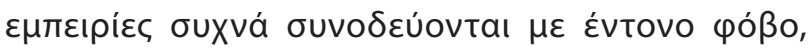

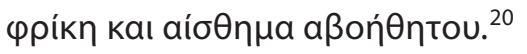

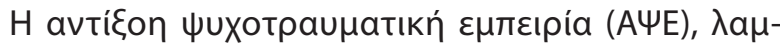

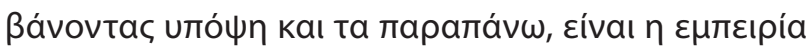

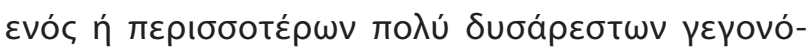

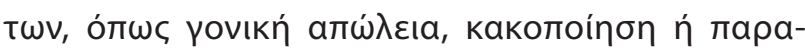

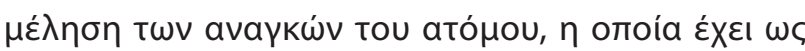

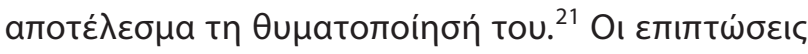

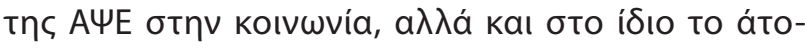

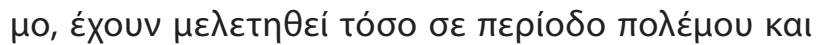

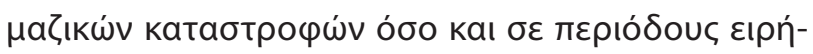

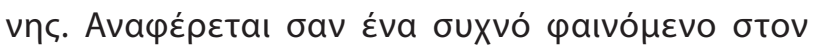

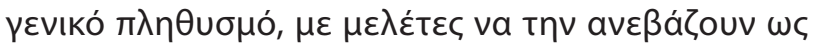

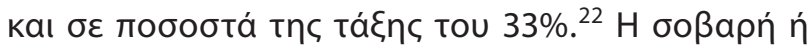

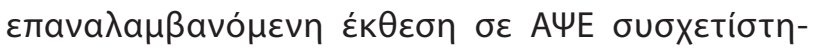

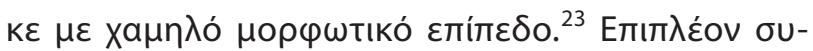

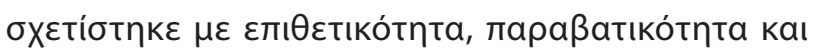

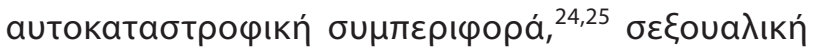

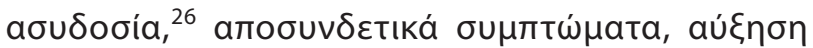

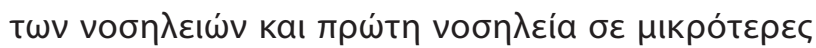

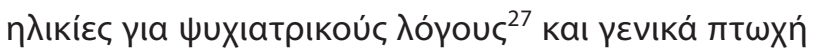

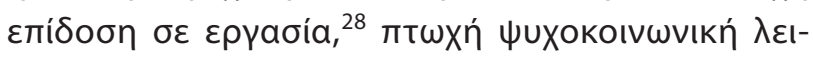

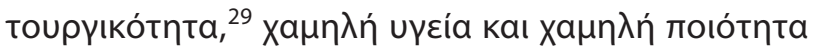

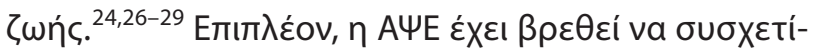

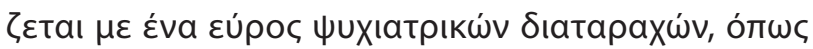

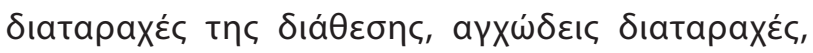

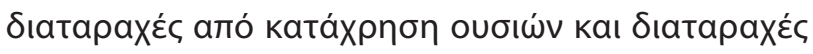

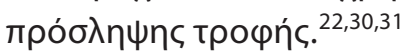




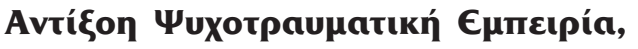

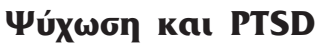

H kaӨı́์

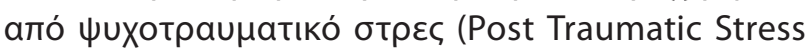

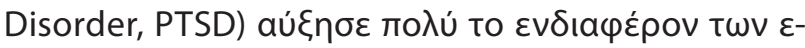

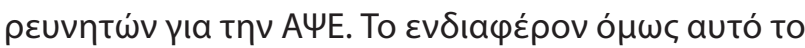

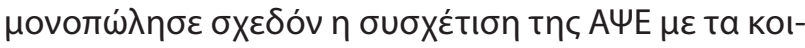

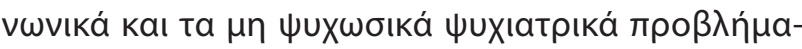

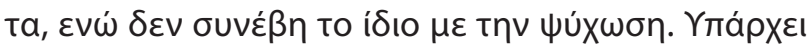

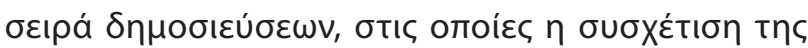

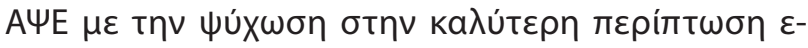

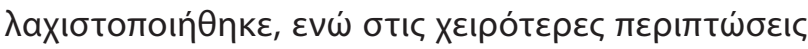

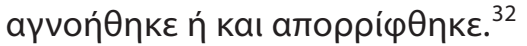

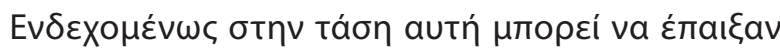

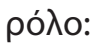

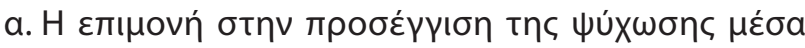

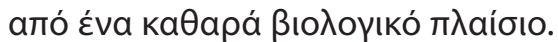

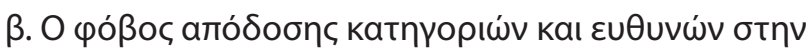

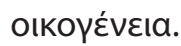

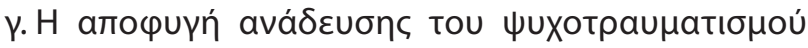

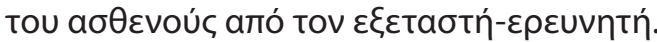

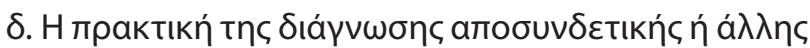

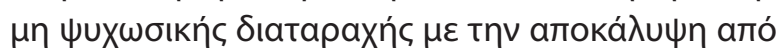

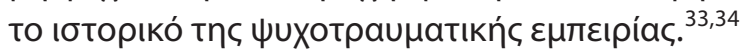

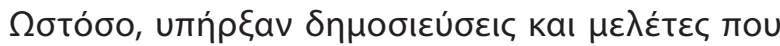

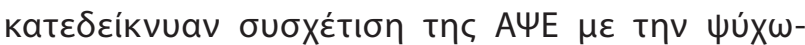

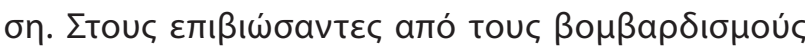

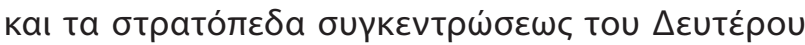

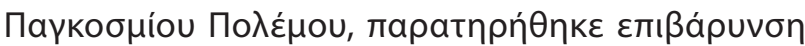

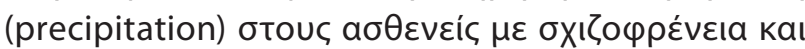

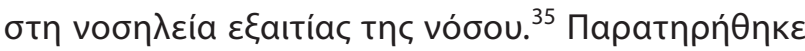

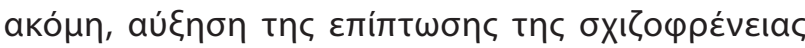
ó

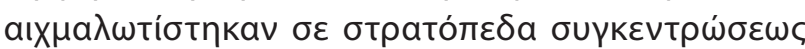

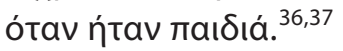

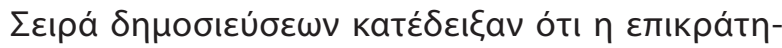

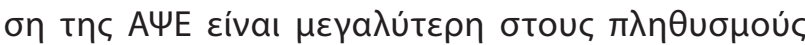

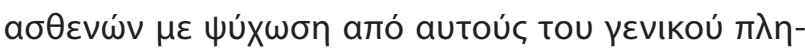

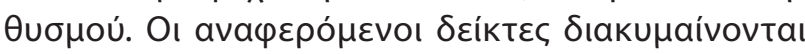

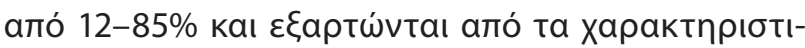

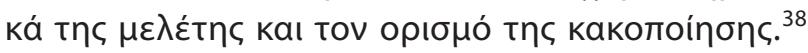

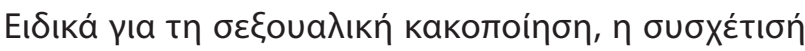

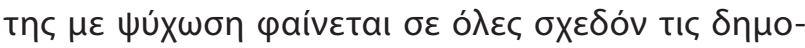

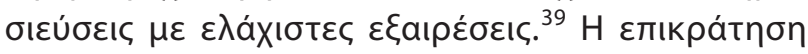

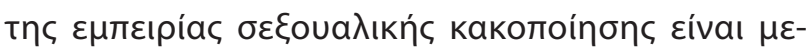

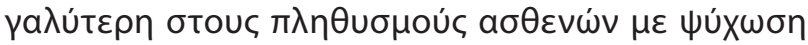

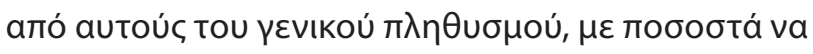

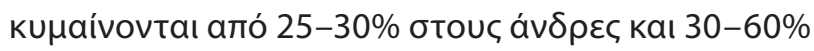
бтıৎ үuvaíkعৎ. ${ }^{40}$

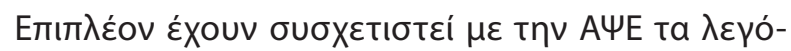

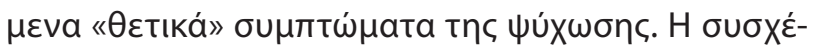

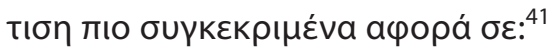

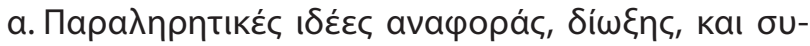

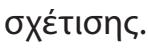

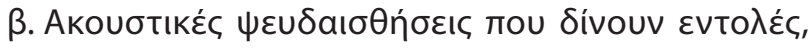

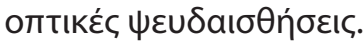

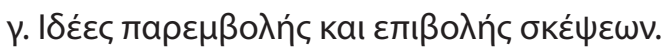

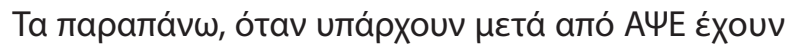

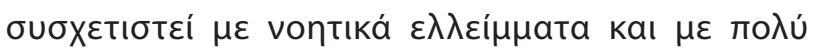

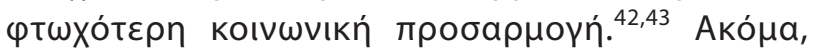

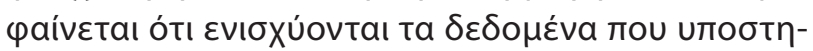

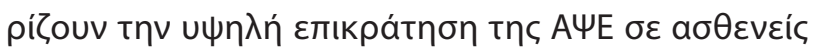

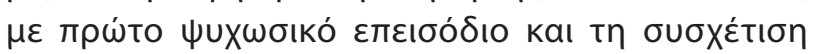

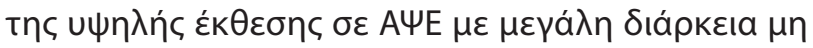

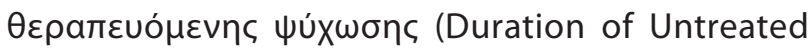
Psychosis, DUP). ${ }^{44}$

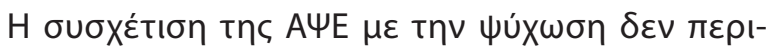

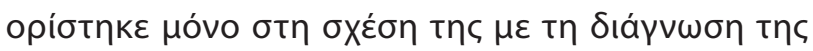

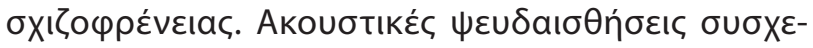

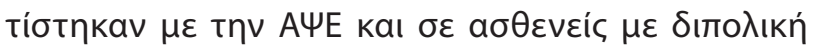

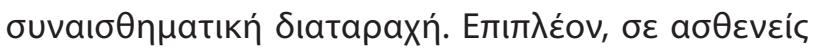

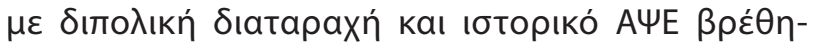

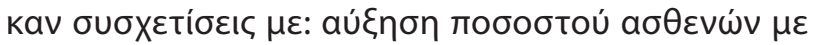

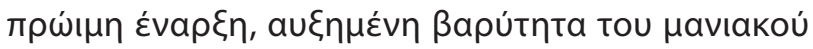

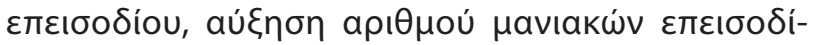

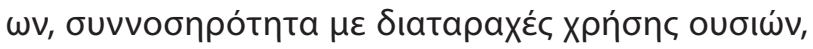

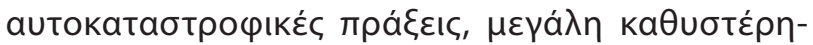

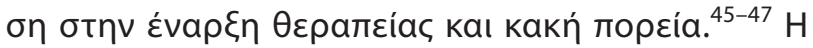

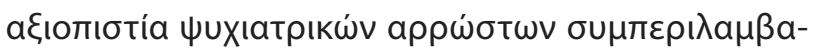

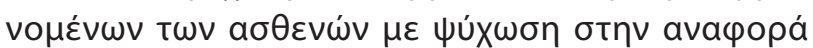

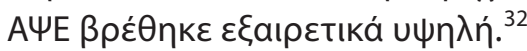

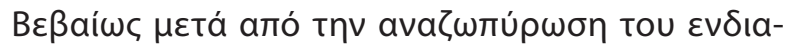

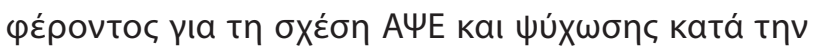

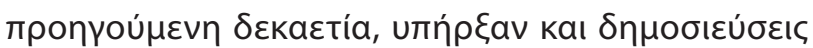

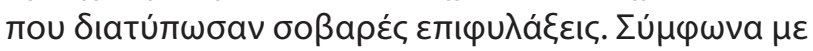

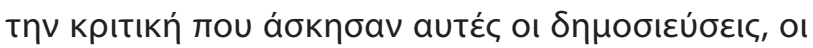

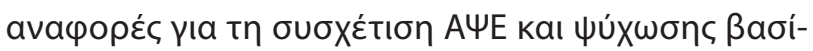

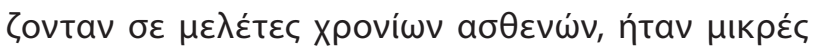

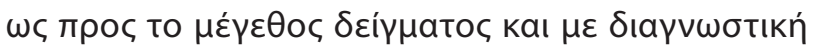

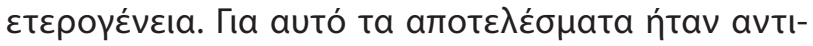

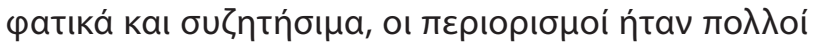

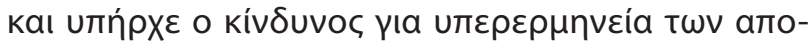




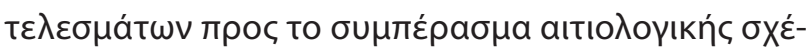

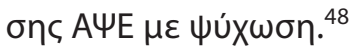

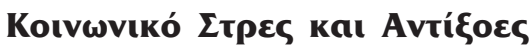

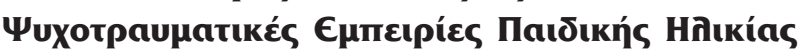

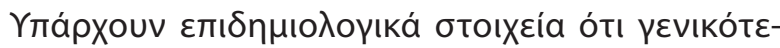

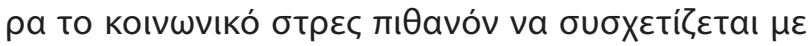

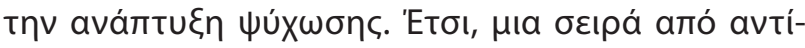

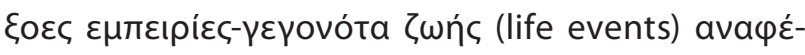

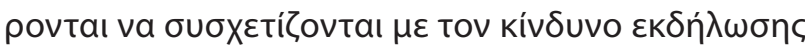

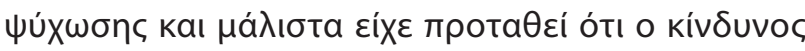

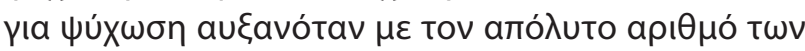

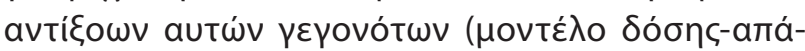
vtnonc, dose-response model). ${ }^{49}$ Ta avtíłoa autá үદүovóta apopoúv:

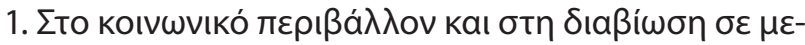

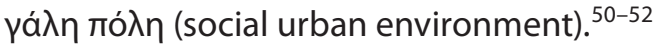

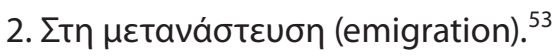

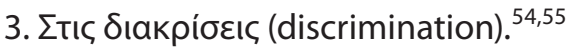

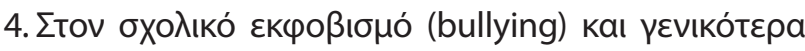

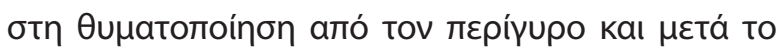

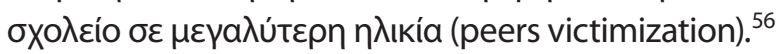

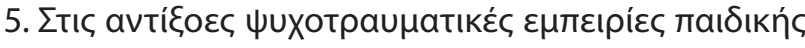

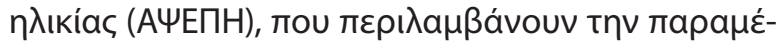

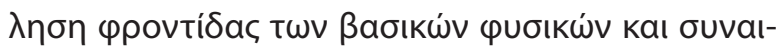

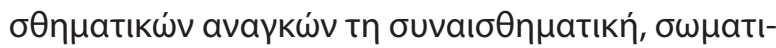

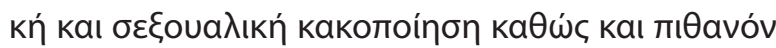

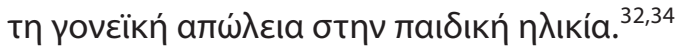

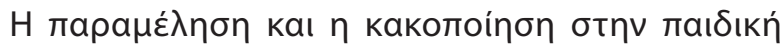

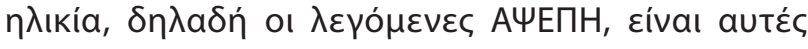

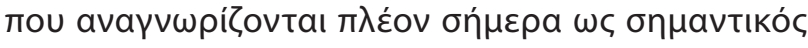

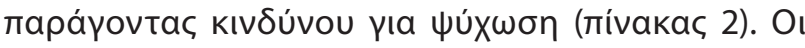

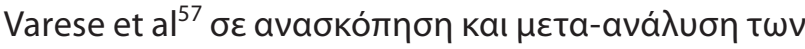

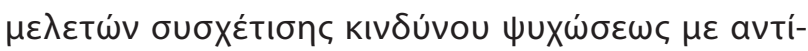

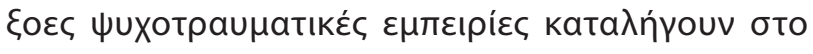

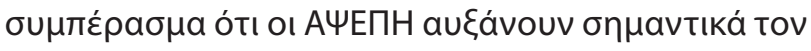

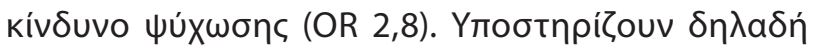

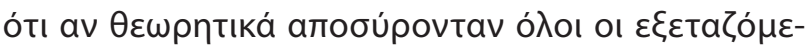

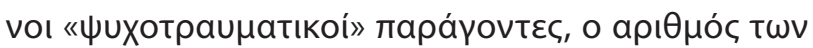

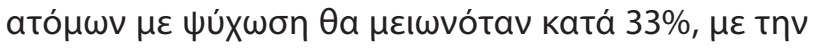

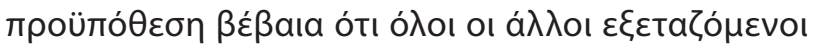

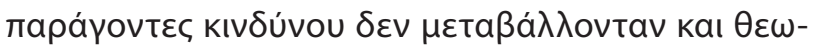

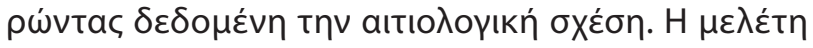

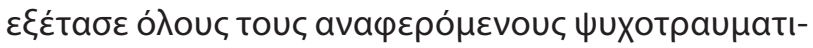

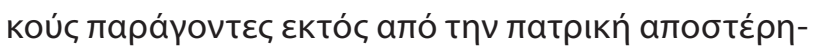

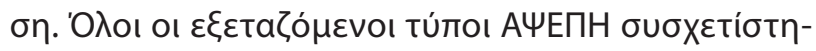

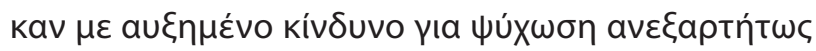

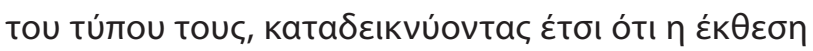

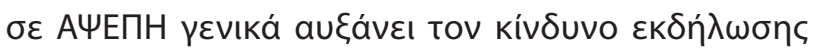

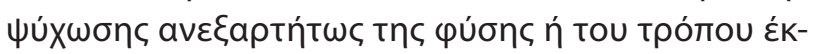

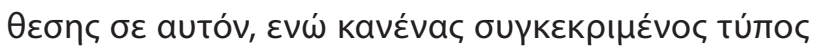

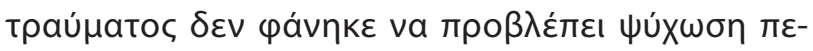

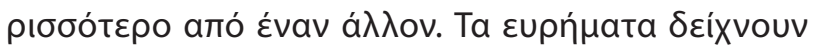

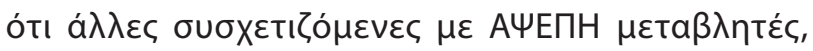

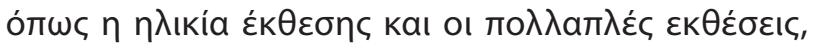

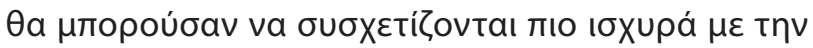

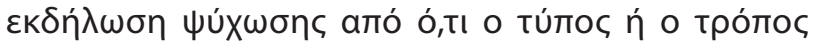

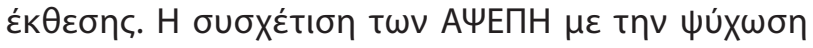

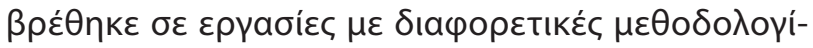

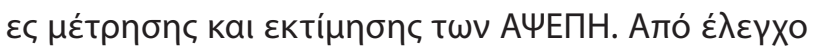

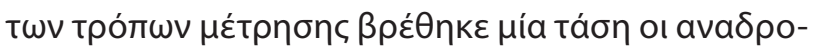

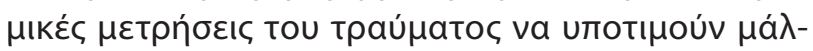

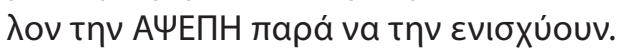

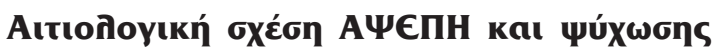

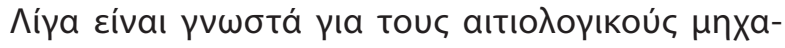

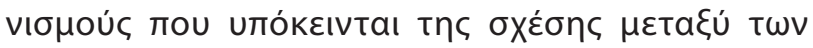

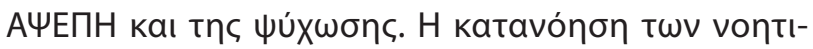

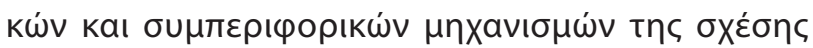

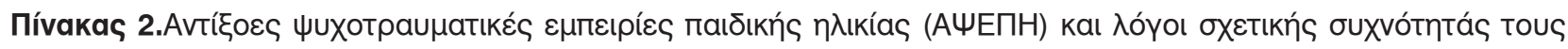

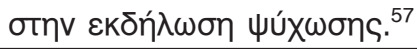

\begin{tabular}{|c|c|c|}
\hline АЧЕПН & Odds Ratio $(95 \% \mathrm{Cl})$ & p \\
\hline 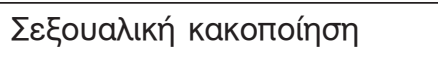 & $2,38(1,98-2,87)$ & $p<0,001$ \\
\hline 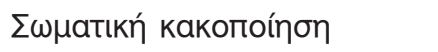 & $2,95(2,25-3,88)$ & $p<0,001$ \\
\hline 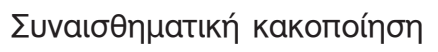 & $3,40(2,06-5,62)$ & $p<0,001$ \\
\hline Екфоßıбно́ & $2,39(1,83-3,11)$ & $p<0,001$ \\
\hline Đávatos yovéa & $1,70(0,82-3,53)$ & $p=0,154$ \\
\hline 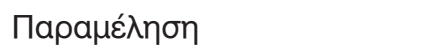 & $2,90(1,71-4,92)$ & $p<0,001$ \\
\hline
\end{tabular}




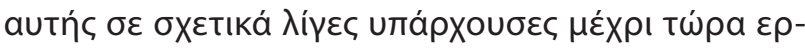

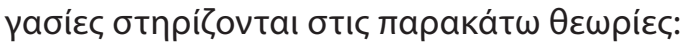

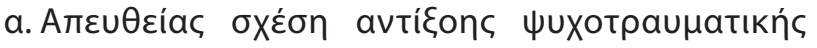

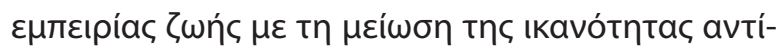

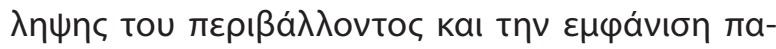

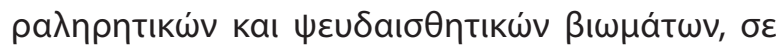

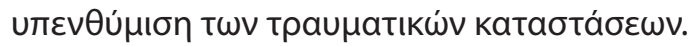

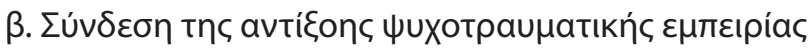

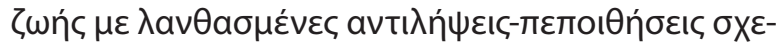

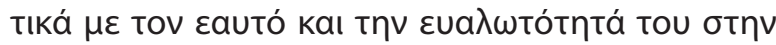

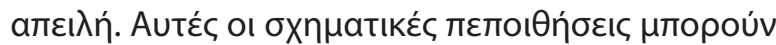

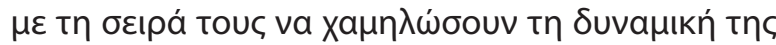

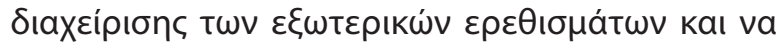

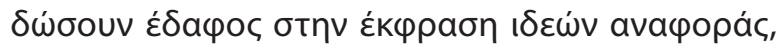

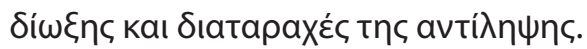

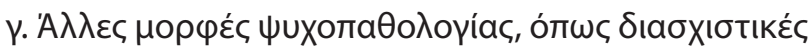

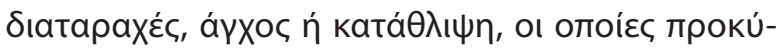

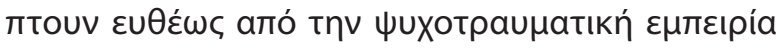

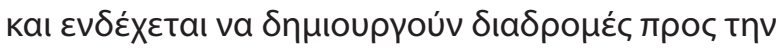
$\psi u ́ x \omega \sigma \eta{ }^{32,58}$

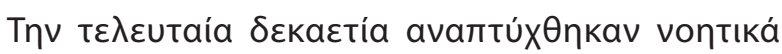

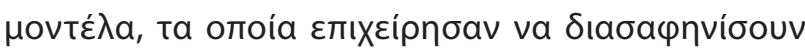

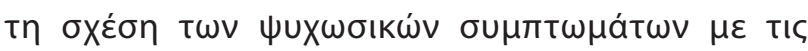

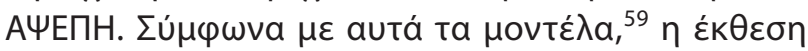

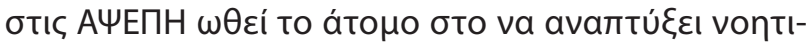

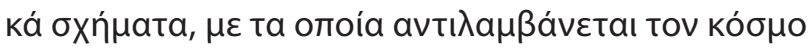

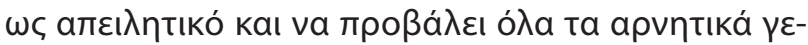

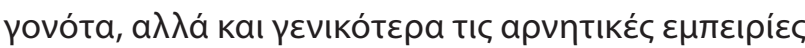

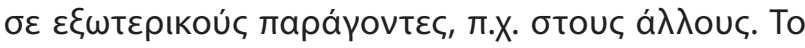

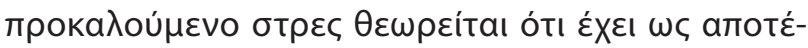

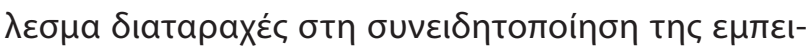

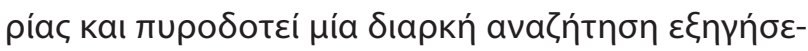

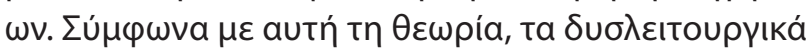

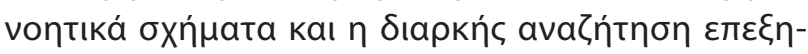

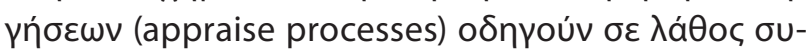

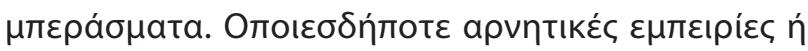

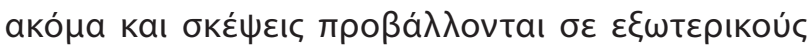

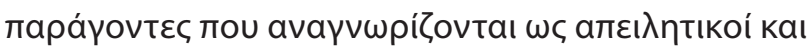

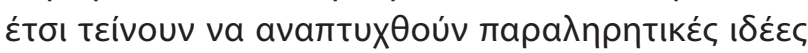

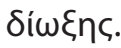

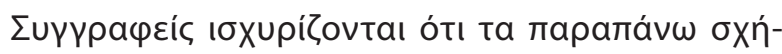

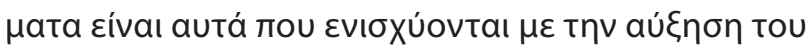

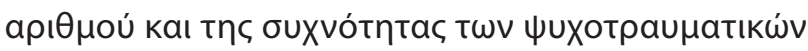

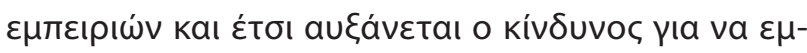

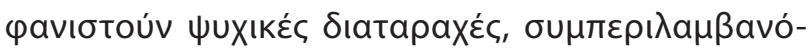

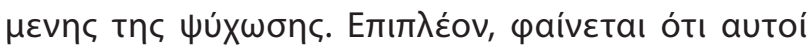

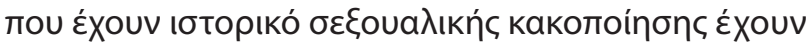

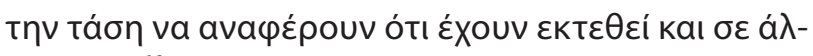

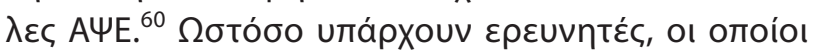

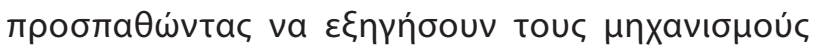

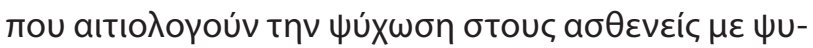

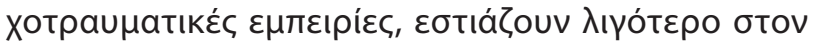

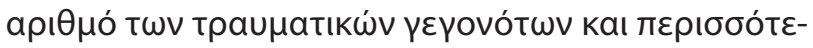

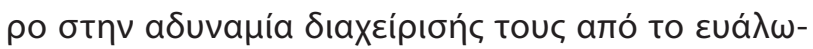

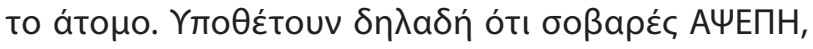

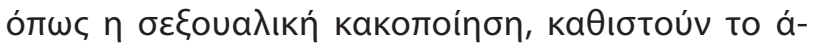

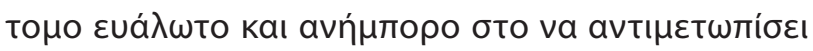

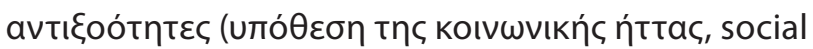

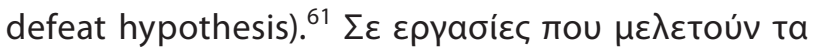

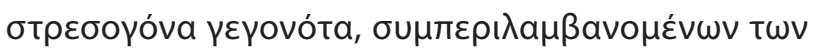

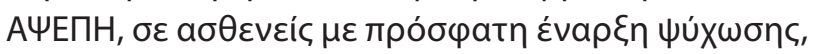

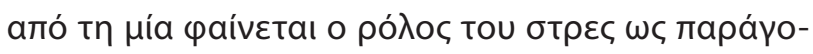

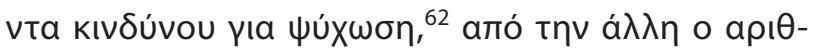

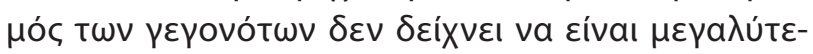

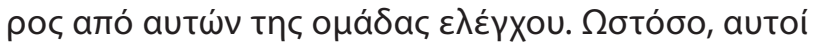

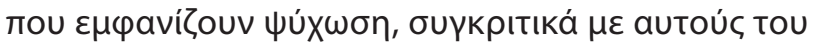

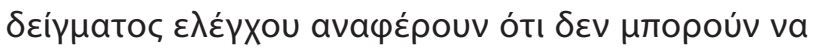

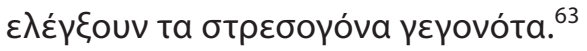

$\sum u ́ \mu \varphi \omega v a \mu \varepsilon$ touç Germeys \& van Os (2007) uтáp-

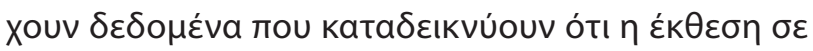

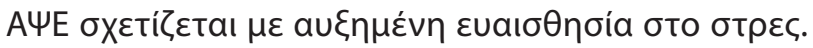

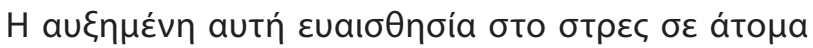

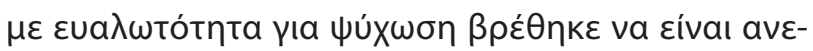

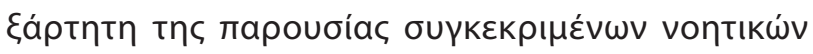

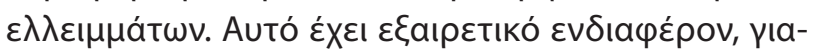

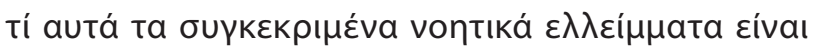

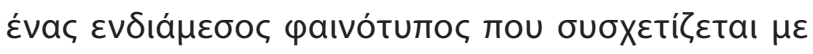

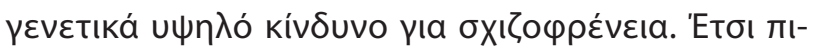

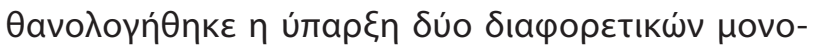

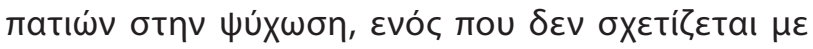

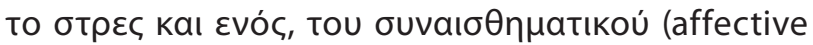

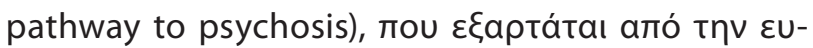

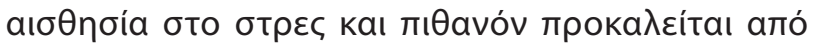
АЧЕПH. ${ }^{64-66}$

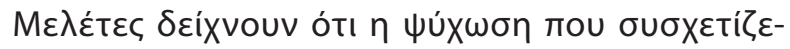

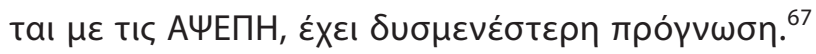

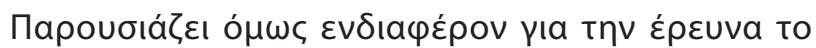

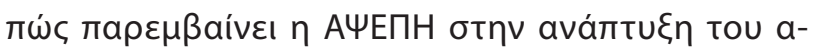

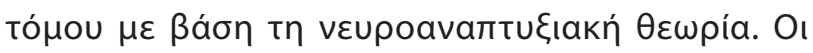

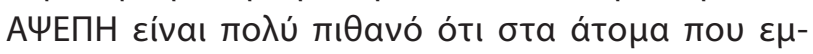

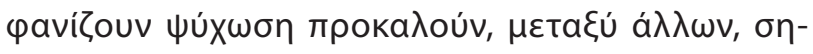

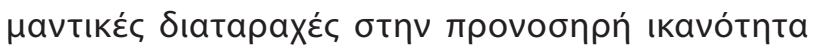

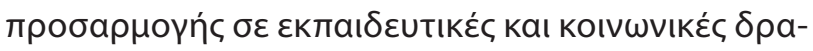

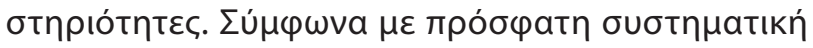




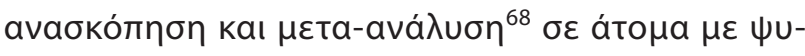

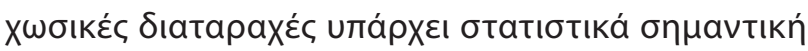
a

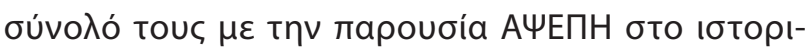

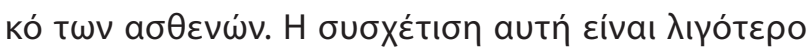

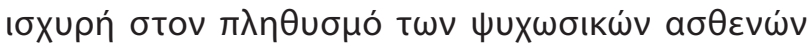

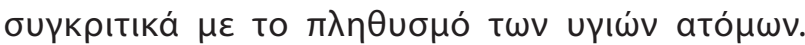

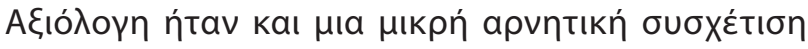

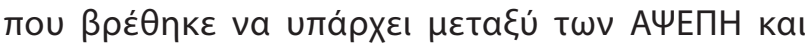

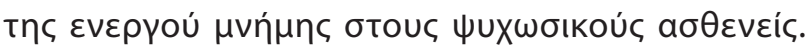

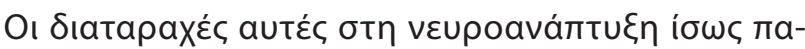

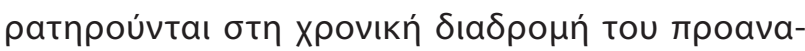

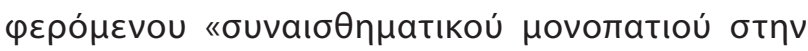

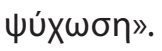

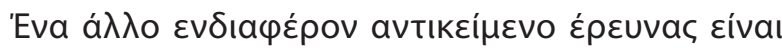

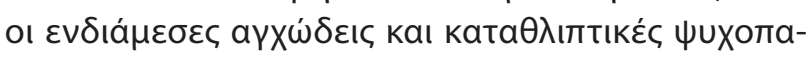

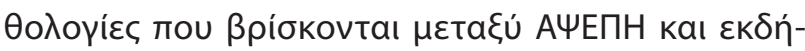

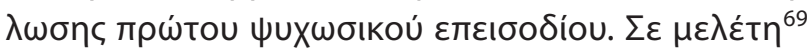

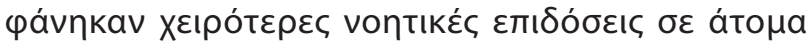

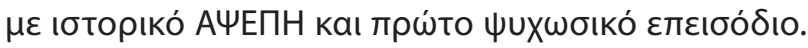

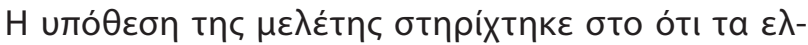

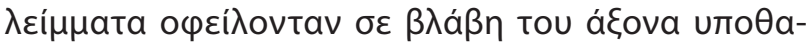

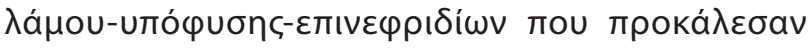

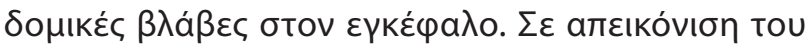

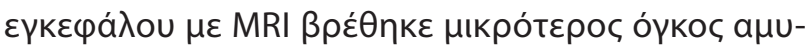

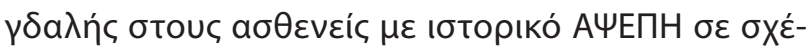

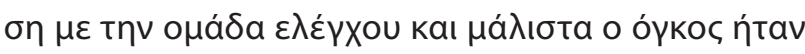

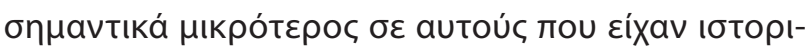

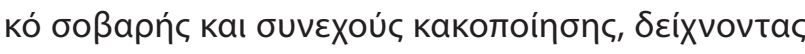

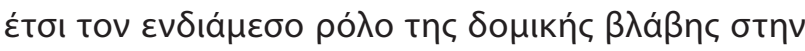

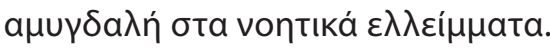

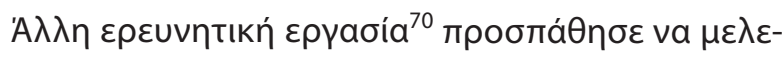

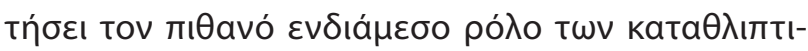

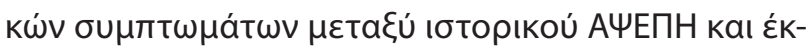

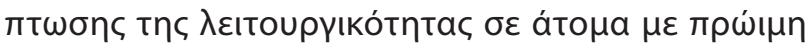

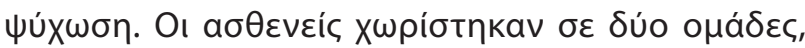

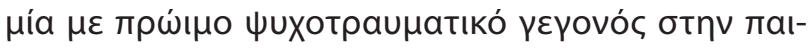

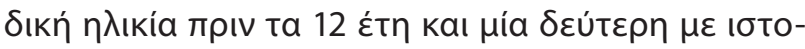

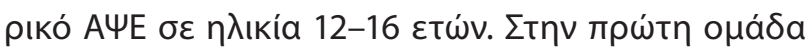

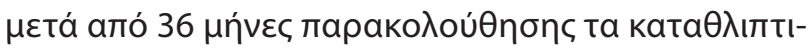

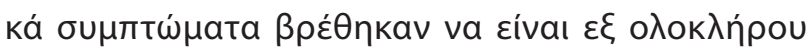

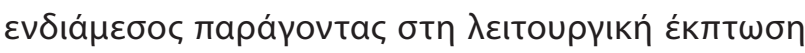
$\tau \omega v \psi \psi u x \omega \sigma ı \kappa \omega ́ v ~ a \sigma \theta \varepsilon v \omega \dot{v}$. Oı Chatziioannidis et $\mathrm{al}^{71}$

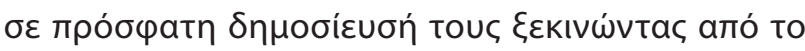

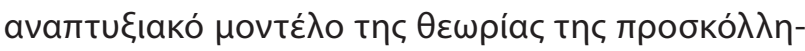

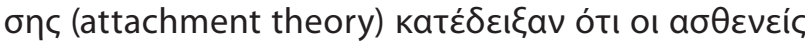

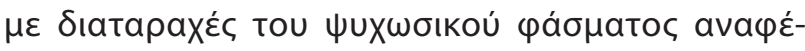

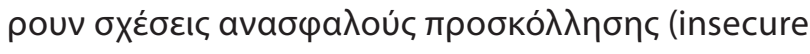

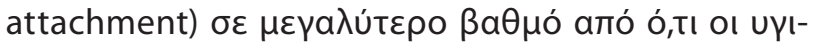

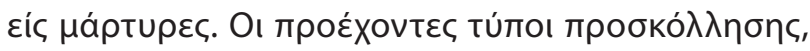

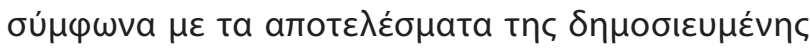

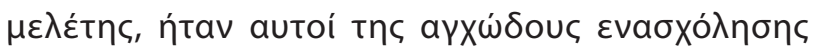

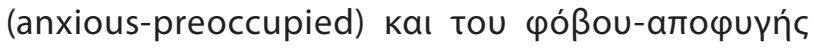

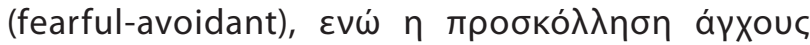

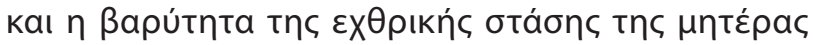

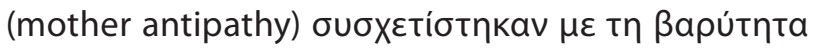

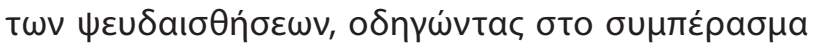

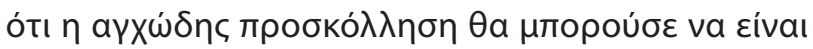

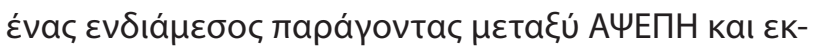

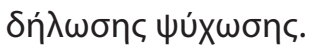

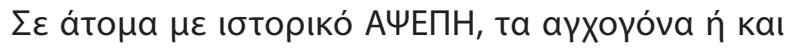

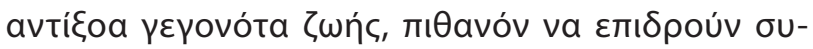

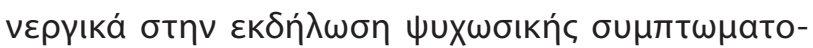

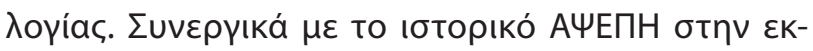

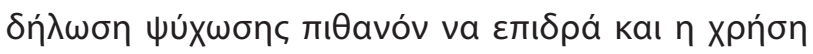

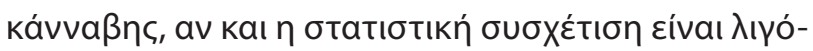

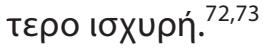

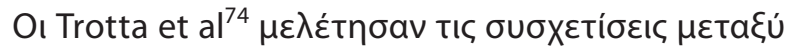

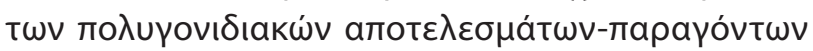
KIVठúvou yıa $\psi u ́ x \omega \sigma \eta$ (PRS, polygenic risk scores)

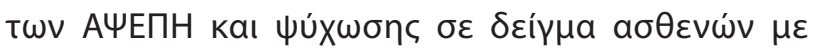

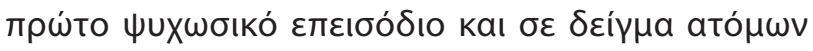

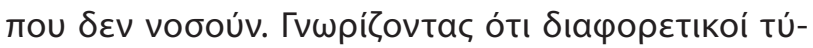

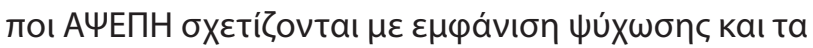

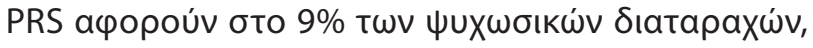

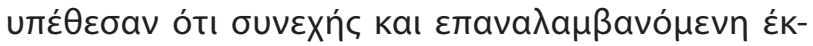

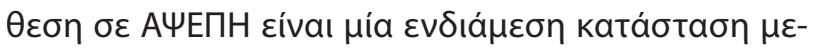

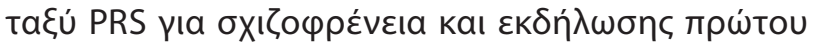

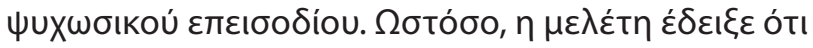

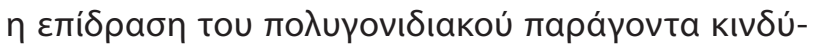

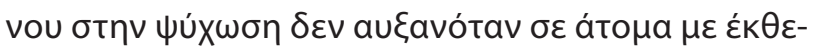

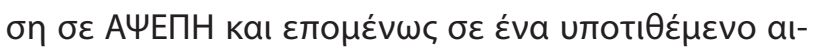

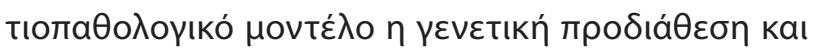

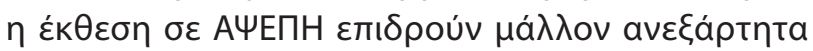
$\mu \varepsilon T a \varepsilon u ́$ Touc.

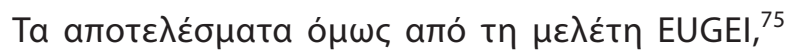

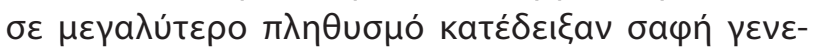

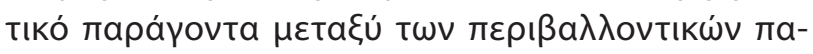

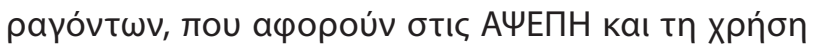

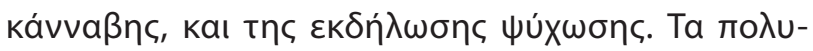

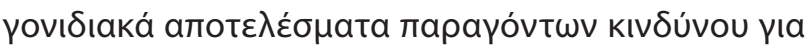

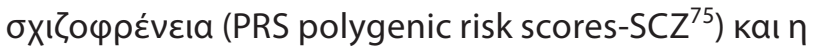

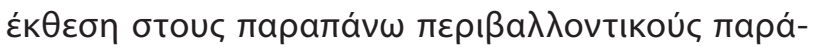

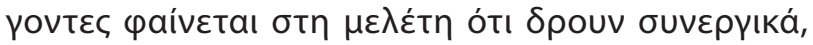




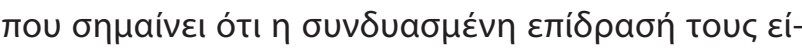

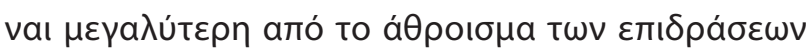

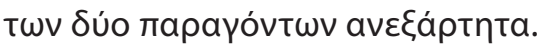

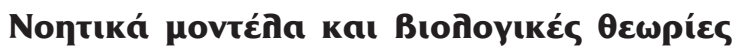

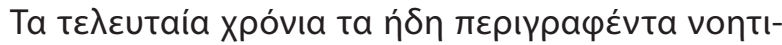

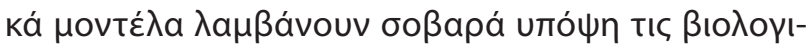

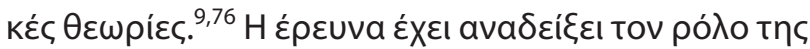

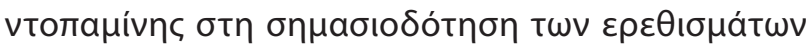

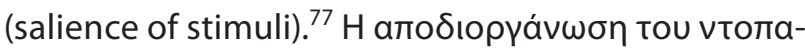

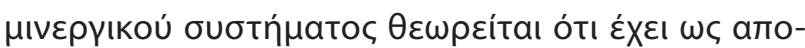

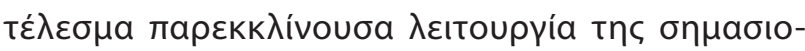

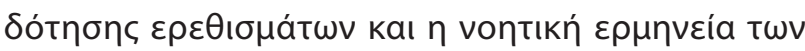

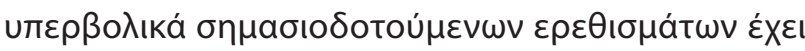

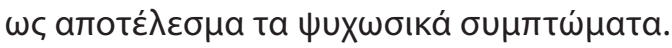

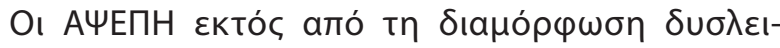

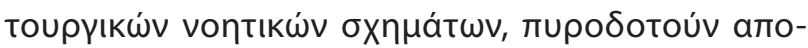

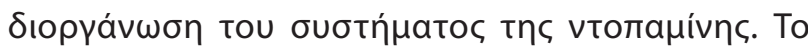

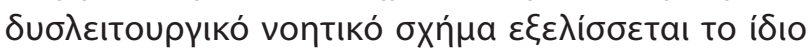

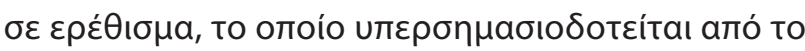

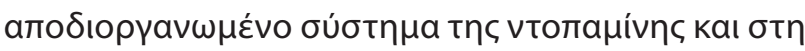

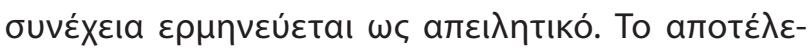

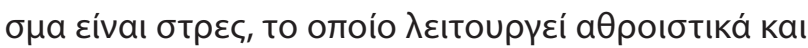

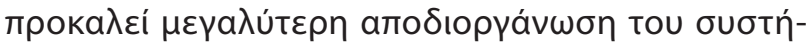

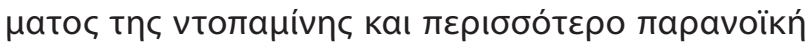

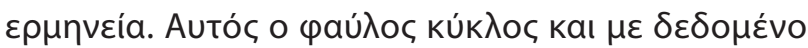

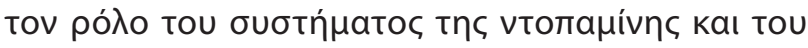

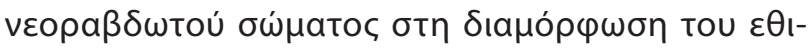

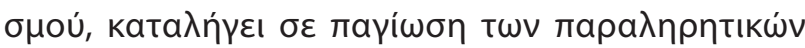

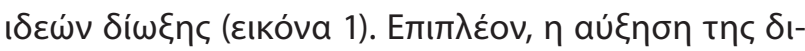

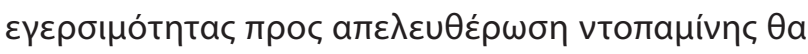

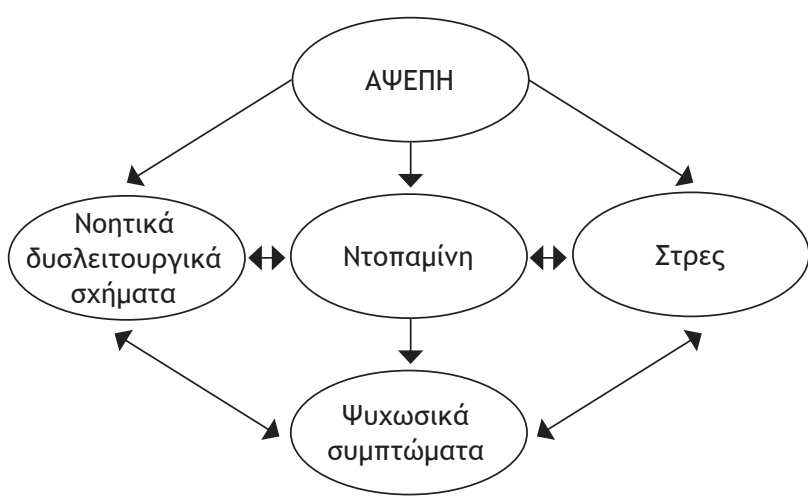

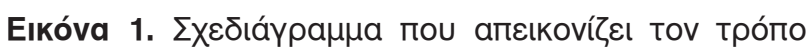

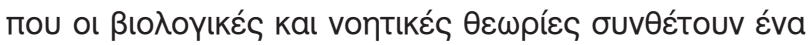

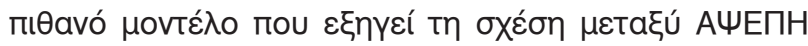
kaı 廿úxwons.

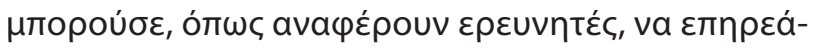

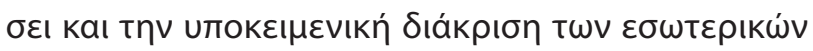

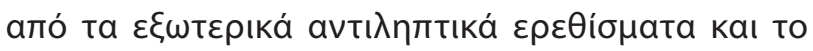

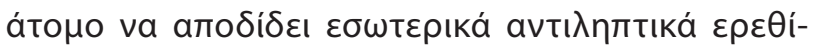

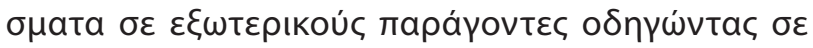

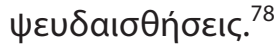

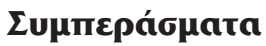

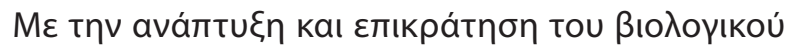

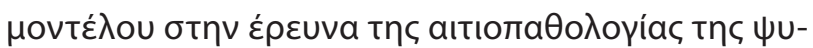

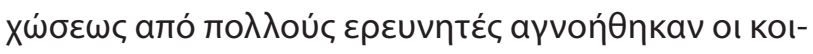

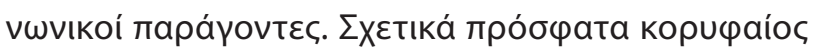

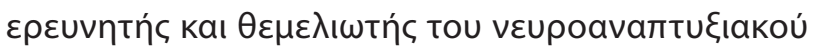

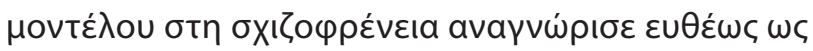

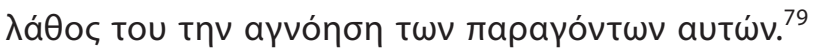

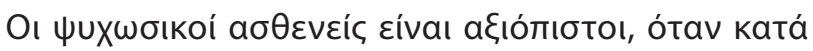

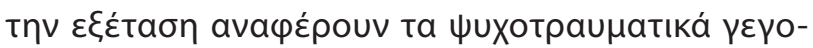

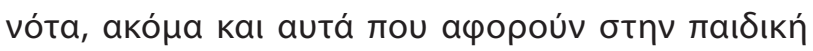

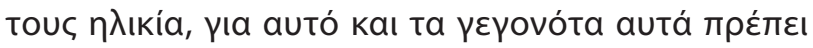

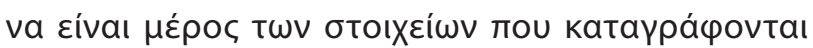

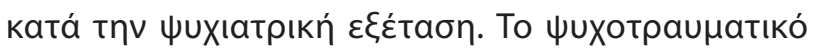

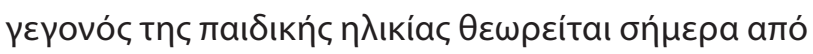

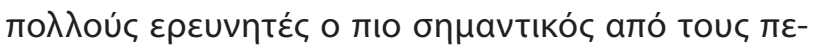

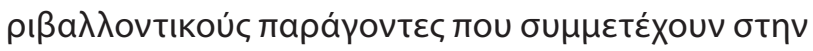

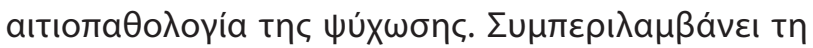

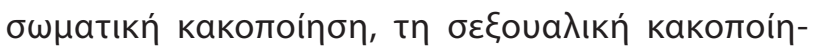

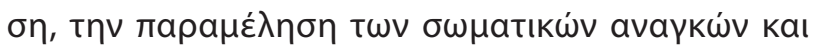

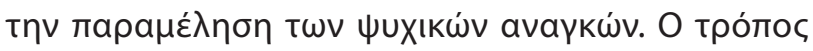

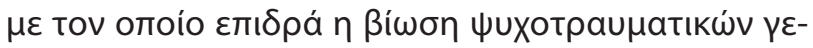

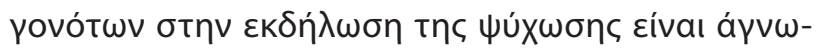

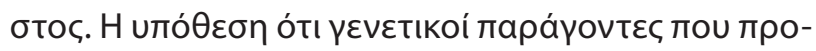

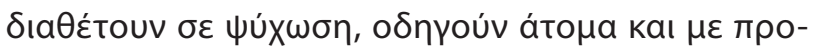

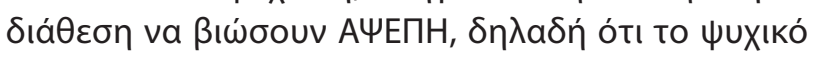

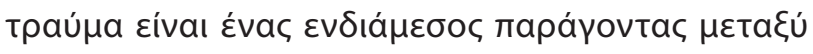

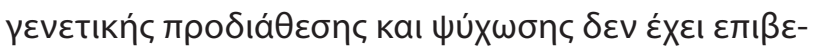

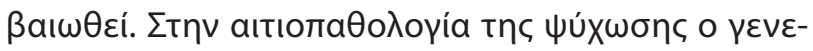

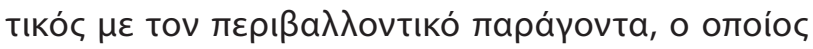

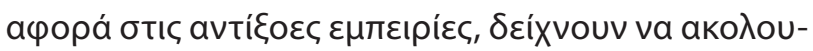

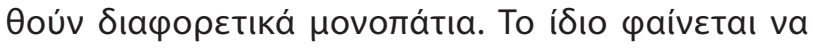

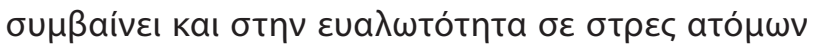

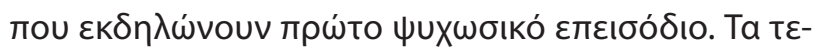

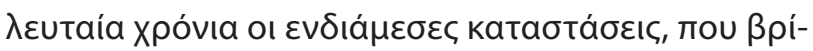

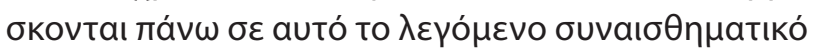

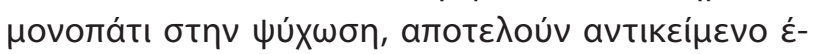

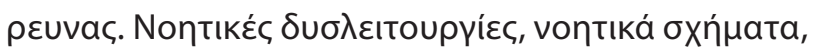

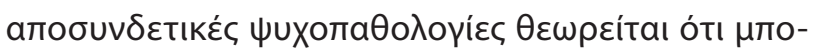

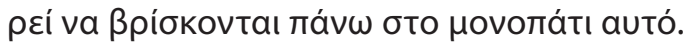




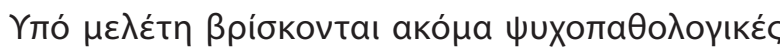

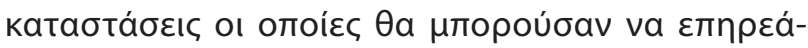

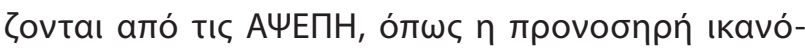

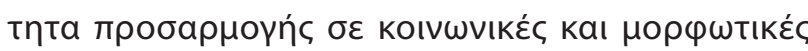

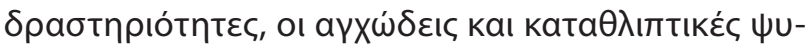

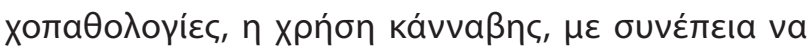

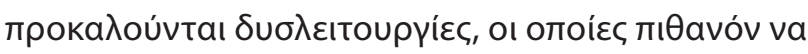

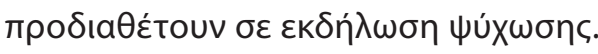

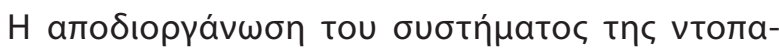

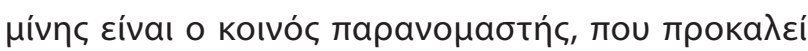

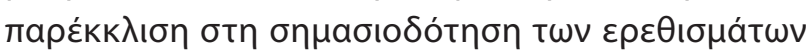

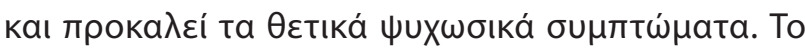

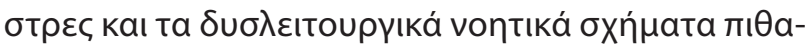

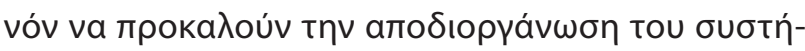

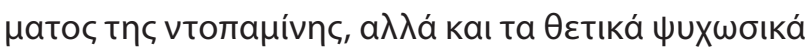

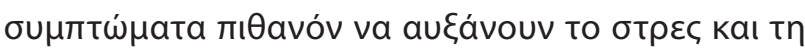

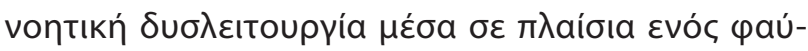

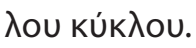

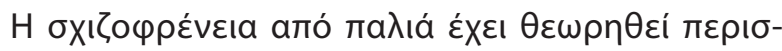

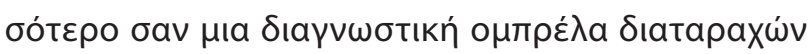

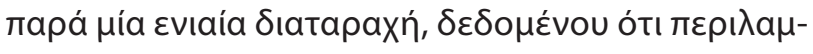

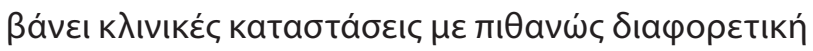

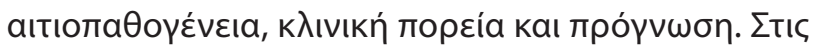

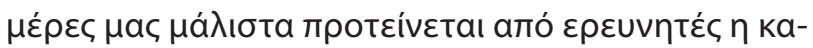

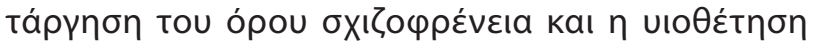

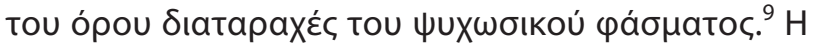

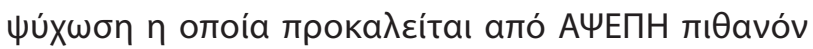

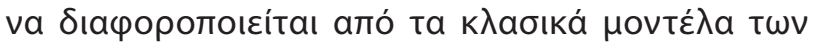

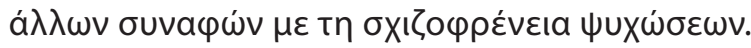

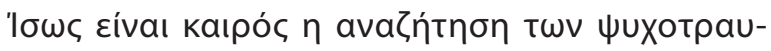

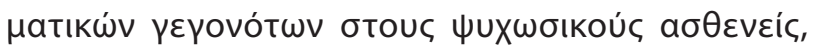

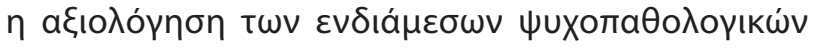

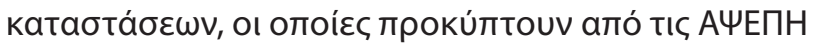

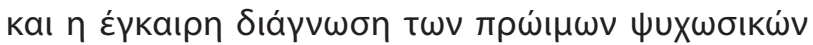

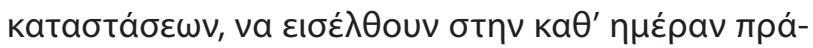

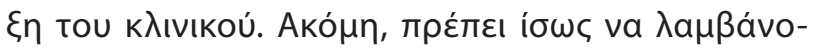

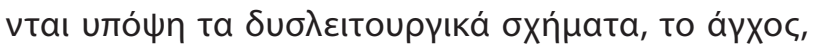

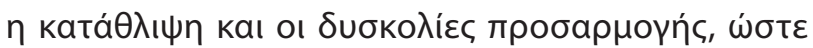

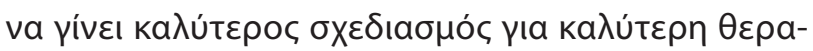

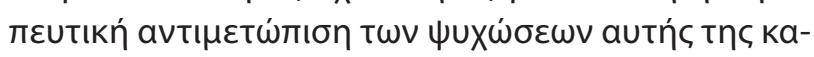
тпүopías.

\title{
Childhood adverse traumatic experiences and schizophrenia
}

\author{
I. Kosteletos, K. Kollias, N. Stefanis \\ 1st Psychiatric Department, University of Athens, Eginition Hospital, Athens, Greece
}

Psychiatriki 2020, 31:23-35

Epidemiological research during last decades has demonstrated the association of certain environmental variables with psychosis. In parallel with understanding environmental contributors to schizophrenia, identification of those at high risk of psychosis has become a priority. These risk factors include adverse traumatic experiences, such as discrimination, social environment adversities, bullying, emigration and childhood trauma. For many years the possibility of a relationship of adverse traumatic experiences with schizophrenia was minimized in favor of a biological paradigm. However, there is evidence supporting that childhood adversities is one of the most significant environmental risk factors for psychosis, if not the most significant. Studies have demonstrated the validity and the reliability of retrospective reports of adverse traumatic experiences in psychotic patients. The question on the causal relationship between adverse traumatic experiences and psychosis has been raised for many years, but it has not yet been answered. Researchers have focused on the study of dysfunctional cognitive schemes being formed due to childhood adversities and 
interpreting the world as unfriendly and threatening. According to social defeat hypothesis, the patients do not experience more stressful life events but they experience them as less controllable. Furthermore, researchers study the mediator psychopathology associated directly with adverse traumatic experiences, such as anxiety, mood, dissociative and adaptation disorders, which possibly predispose to psychosis. Cannabis use and childhood adversities may interact to increase the risk of psychotic symptoms in adolescence, according to research findings. Several publications have positively linked the number of traumatic adverse experiences with the severity of mental health and possibly with high risk of psychosis in a dose-response relationship model. Taking note that it is important to have a plausible hypothesis of the biological mechanisms involved with the relationship between adverse traumatic experiences and psychosis, the putative systems being explored are: (1) the hypothalamus-pituitary-adrenal (HPA) axis because it mediates the principal adaptive response to perceived psychological or physiological stress and (2) the dopamine system, which is considered to be important in the development of psychosis. The biological and environmental risk factors are both important in the etiology of psychosis but the effects of some forms of childhood adversity act largely independently of pre-existing genetic liability to increase the risk of psychosis. A supposed differentiation of psychosis associated with adverse traumatic experiences from the classic neurodegenerative model possibly should be considered seriously by the clinician in order to design the best therapeutic plan.

Key words: First episode psychosis, schizophrenia, trauma, adversities.

\section{BıßAtoypacpía}

1. Salomon JA, Vos T, Hogan DR et al. Common values in assessing health outcomes from disease and injury: disability weights measurement study for the Global Burden of Disease Study 2010. Lancet 2012, 380:2129-2243, doi: 10.1016/S01406736(12)61680-8

2. Perälä J, Suvisaari J, Saarni SI, Kuoppasalmi K, Isometsä E, Pirkola $S$ et al. Lifetime prevalence of psychotic and bipolar I disorders in a general population. Arch Gen Psychiatry 2007, 64:19-28, doi: 10.1017/S0033291712001626

3. Golokzuz S, van Os J. The slow death of the concept of schizophrenia and the painful birth of the psychosis spectrum. Psychol Medicine 2018, 48:229-244, doi: 10.1017/S0033291717001775

4. Vos T, Barber RM, Bell B, Bertozzi-Villa A, Biryukov S, Bolliger I et al. Global, regional, and national incidence, prevalence, and years lived with disability for 301 acute and chronic diseases and injuries in 188 countries, 1990-2013: A systematic analysis for the Global Burden of Disease Study 2013. Lancet 2015, 386:743-800, doi: 10.1016/S0140-6736(15)60692-4

5. Jongsma HE, Gayer-Anderson C, Lasalvia A, Quattrone D, Mulè A, Szöke A et al. European Network of National Schizophrenia Networks Studying Gene-Environment Interactions Work Package 2 (EU-GEI WP2) Group. Treated Incidence of Psychotic Disorders in the Multinational EU-GEI Study. JAMA Psychiatry 2018, 75:36-46, doi:10.1001/jamapsychiatry.2017.3554

6. Peritogiannis V, Mantas C, Tatsioni A, Mavreas V. Rates of first episode of psychosis in a defined catchment area in Greece. Clin Pract Epidemiol Ment Health 2013, 9:251-254, doi: $10.2174 / 1745017901309010251$

7. Leucht S, Tardy M, Komossa K, Heres S, Kissling W, Salanti G et al. Antipsychotic drugs versus placebo for relapse prevention in schizophrenia: a systematic review and meta-analysis. Lancet 2012, 379:2063-2071, doi: 10.1016/S0140-6736(12)60239-6

8. Howes OD, Egerton A, Allan V, McGuire P, Stokes P, Kapur S. Mechanisms underlying psychosis and antipsychotic treatment response in schizophrenia: insights from PET and SPECT imaging. Curr Pharm Des 2009, 15:2550-2559, doi: $10.2174 / 138161209788957528$

9. Howes OD, Murray RM. Schizophrenia: an integrated sociodevelopmental-cognitive model. Lancet 2014, 383:1677-1687, doi: 10.1016/S0140-6736(13)62036-X

10. Schizophrenia Working Group of the Psychiatric Genomics Consortium. Biological insights from 108 schizophrenia associated genetic loci. Nature 2014, 511:421-427, doi:10.1038/ nature 13595

11. Zhang JP, Lencz LF, Geisler S, DeRosse P, Bromet EJ, Malhotra AK. Genetic variation in BDNF is associated with antipsychotic treatment resistance in patients with schizophrenia. Schizophr Res 2013, 146:285-288, doi: 10.1016/j.schres.2013.01.020

12. Goldstein JI, Jarskog LF, Hilliard C, Alfirevic A, Duncan L, Fourches $D$ et al. Clozapine induced agranulocytosis is associated with rare HLA-DQB1 and HLB alleles. Nat Commun 2014, 5:4757, doi:10.1038/ncomms5757

13. O'Donovan MC, Craddock N, Owen MJ. Schizophrenia: complex genetics, not fairy tales. Psychol Med 2008, 38:1697-1699, doi: $10.1017 /$ S0033291708003802

14. Crow TJ. The emperors of the schizophrenia polygene have no clothes. Psychol Med 2008, 38:1681-1685, doi:10.1017/ S0033291708003395.

15. van Os J, Kenis G, Rutten BP. The environment and schizophrenia. Nature 2010, 468:203-212, doi: 10.1038/nature09563 
16. Padmanabhan JL, Shah JL, Neeraj T, Matcheri KS The "polyenviromic risk score": Aggregating environmental risk factors predicts conversion to psychosis in familial high-risk subjects. Schizophr Res 2017, 181:17-22, doi: 10.1016/j.schres. 2016.10.014

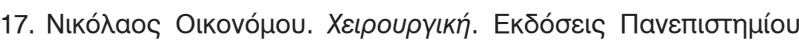
AӨnvẃv, AӨńva, 1978:144

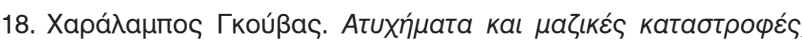

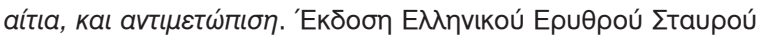

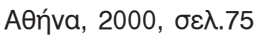

19. "Trauma Definition". Substance Abuse and Mental Health Services Administration. Archived from the original on August 5, 2014. http://www.samhsa.gov/traumajustice/traumadefinition/ definition.aspx

20. American Psychiatric Association. Diagnostic and statistical manual of mental disorders. 4th ed. Text revision. Washington, DC, 2000 , doi: 10.1176/appi.books.9780890423349

21. Nelson EC, Heath AC, Madden PAF, Cooper ML, Dinwiddie SH, Bucholz KK et al. Association between self-reported childhood sexual abuse and adverse psychosocial outcomes. results from a twin study. Arch Gen Psychiatry 2002, 59:139-145, doi:10.1001/archpsyc.59.2.139

22. Kessler RC, McLaughlin KA, Green JG, Gruber MJ, Sampson NA, Zaslavsky AM et al. Childhood adversities and adult psychopathology in the WHO World Mental Health Surveys. Br J Psychiatry 2010, 197:378-385, doi: 10.1192/bjp.bp.110.080499

23. Mullen P, Martin J, Anderson J, Romans S, Herbison G. Childhood sexual abuse and mental health in adult life. $\mathrm{Br} J$ Psychiatry 1993, 163:721-732, doi: 10.1192/bjp.163.6.721

24. Bushnell J, Wells J, Oakley-Browne M. Long-term effects of intrafamilial sexual abuse in childhood. Acta Psychiatr Scand 1992, 85:136-142, doi:10.1111/j.1600-0447.1992.tb01458.x

25. Christoffersen M, Poulsen H, Nielsen A. Attempted suicide among young people: risk factors in a prospective register based study of Danish people born in 1966. Acta Psychiatr Scand 2003, 108:350-358, PMID:14531755

26. Kim D, Kaspar V, Noh S, Nam JH. Sexual and physical abuse among Korean female inpatients with schizophrenia. J Trauma Stress 2006, 19:279-289, doi:10.1002/jts.20119

27. Schenkel LS, Spaulding WD, Dilillo D, Silverstein SM. Histories of childhood maltreatment in schizophrenia: relationships with premorbid functioning, symptomatology and cognitive deficits. Schizophr Res 2005, 76:273-286, doi: 10.1016/j. schres.2005.03.003

28. Lysaker PH, Meyer PS, Evans JD, Clements CA, Marks KA. Childhood sexual trauma and psychosocial functioning in adults with schizophrenia. Psychiatr Serv 2001, 52:1485-1488, doi: 10.1176/appi.ps.52.11.1485

29. Lysaker PH, Nees MA, Lancaster RS, Davis LW. Vocational function among persons with schizophrenia with and without history of childhood sexual trauma. J Trauma Stress 2004, 17:435-438, doi:10.1023/B:JOTS.0000048957.70768.b9

30. Kendler KS, Bulik CM, Silberg J, Hettema JM, Myers J, Prescott CA. Childhood sexual abuse and adult psychiatric and substance use disorders in women. An epidemiological and cotwin control analysis. Arch Gen Psychiatry 2000, 57:953-959, doi:10.1001/archpsyc.57.10.953

31. Grilo CM, Masheb RM. Childhood maltreatment and personality disorders in adult patients with binge eating disorder. Acta Psychiatr Scand 2002, 106:183-188, doi: 10.1034/j.1600-0447. 2002.02303.x

32. Read J, van Os J, Morrison AP, Ross CA. Childhood trauma, psychosis and schizophrenia: a literature review with theoretical and clinical implications. Acta Psychiatr Scand 2005, 112: 330-350, doi: 10.1111/j.1600-0447.2005.00634.x

33. Read J. Child Abuse and psychosis: a literature review and implications for professional practice. Prof Psychol Res $\operatorname{Pr} 1997$ 28:448-456, doi: 10.1037/0735-7028.28.5.448

34. Bendall S, Jackson HJ, Hulbert CA, McGorry PD. Childhood trauma and psychotic disorders: a systematic, critical review of the evidence. Schizophr Bull 2008, 34:568-579, doi: 10.1093/ schbul/sbm121

35. Steinberg HR, Durell J. A stressful social situation as a precipitant of schizophrenic symptoms: an epidemiological study. Br J Psychiatry 1968, 114:1097-1105, doi: 10.1192/bjp. 114.514.1097

36. Luchterhand E. Early and late effects of imprisonment in Nazi concentration camps: Conflicting interpretations in survivor research. Soc Psychiatry 1970, 5:102-110, doi: 10.1007/ BF00594722

37. Reulbach U, Bleich S, Biermann T, Pfahlberg A, Sperling W. Late-Onset Schizophrenia in Child Survivors of the Holocaust. J Nerv Ment Dis 2007, 195:315-319, doi: 10.1097/01.nmd. 0000261878.55882 .55

38. Ucok A, Bikmaz S. The effects of childhood trauma in patients with first episode schizophrenia. Acta Psychiatr Scand 2007, 116:371-377, doi:10.1111/j.1600-0447.2007.01079.x

39. Spataro J, Mullen PE, Burgess PM, Wells DL, Moss A. Impact of child sexual abuse on mental health: prospective study in males and females. Br J Psychiatry 2004, 184:416-421, PMID:15123505

40. Lysaker PH, Beattie BA, Strasburger MA, Davis LW. Reported history of child sexual abuse in schizophrenia. Association with heightened symptom levels and poorer participation over four months in vocational rehabilitation. J Nerv Ment Dis 2005, 193:790-795, PMID: 16319700

41. Ross C, Anderson G, Clark P. Childhood abuse and positive symptoms of schizophrenia. Hosp Community Psychiatry 1994, 164:831-834, PMID:8045548

42. Read J, Perry BD, Miskowitz A. The contribution of early traumatic events to schizophrenia in some patients: a traumatogenic neurodevelopmental model. Psychiatry 2001, 64:319-345, doi: 10.1521/psyc.64.4.319.18602

43. Lysaker PH, Meyer P, Evans JD, Marks KA. Neurocognitive and symptom correlates of self-reported childhood sexual abuse in schizophrenia spectrum disorders. Ann Clin Psychiatry 2001, 13:89-92, PMID:11534930

44. Conus P, Cotton S, Schimmelmann BG, McGorry PD, Lambert M. Pretreatment and Outcome Correlates of Sexual and Physical Trauma in an Epidemiological Cohort of First-Episode 
Psychosis Patients. Schizophr Bull 2010, 36:1105-1114, doi: 10.1093/schbul/sbp009

45. Leverich GS, McElroy SL, Suppes T, Keck PE Jr, Denicoff KD, Nolen WA et al. Early physical and sexual abuse associated with an adverse course of bipolar illness. Biol Psychiatry 2002, 51:288-297, doi: 10.1016/s0006-3223(01)01239-2

46. Garno JL, Goldberg JF, Ramirez PM, Ritzler BA. Impact of childhood abuse on the clinical course of bipolar disorder. $\mathrm{Br}$ J Psychiatry 2005, 186:121-125, doi:10.1192/bjp.186.2.121

47. Leverich GS, Post RM. Course of bipolar illness after history of childhood trauma. Lancet 2006, 367:1040-1042, doi:10.1016/ S0140-6736(06)68450-X

48. Morgan C, Fisher H. Environmental factors in schizophrenia: childhood trauma - a critical review. Schizophr Bull 2007, 33:310, doi: 10.1093/schbul/sbl053

49. Shevlin M, Houston JE, Dorahy MJ, Adamson G. Cumulative traumas and psychosis: An analysis of the national comorbidity survey and the British Psychiatric Morbidity Survey. Schizophr Bull 2008, 34:193-199, doi: 10.1093/schbul/sbm069

50. Van Os J, Hanssen M, Bak M, Bijl RV, Vollebergh W. Do urbanicity and familial liability coparticipate in causing psychosis? Am J Psychiatry 2003, 160:477-482, doi: 10.1176/appi.ajp. 160.3.477

51. Van Os J. Does the urban environment cause psychosis? $\mathrm{Br}$ J Psychiatry 2004, 184:287-288, PMID:15056569

52. Van Os J, Pedersen CB, Mortensen PB. Confirmation of synergy between urbanicity and familial liability in the causation of psychosis. Am J Psychiatry 2004, 161:2312-2314, doi: 10.1176/ appi.ajp.161.12.2312

53. Cantor-Graae E, Selten JP. Schizophrenia and migration: a meta-analysis and review. Am J Psychiatry 2005, 162:12-24, doi:10.1176/appi.ajp.162.1.12

54. Hoek HW. Discrimination and the incidence of psychotic disorders among ethnic minorities in The Netherlands. Int $J$ Epidemiol 2007, 36:761-768, doi: 10.1093/ije/dym085

55. Janssen I, Hanssen M, Bak M et al. Discrimination and delusional ideation. Br J Psychiatry 2003, 182:71-76, PMID: 12509322

56. Trotta A, Di Forti M, Mondelli V, Dazzan P, Pariante C, David A et al. Prevalence of bullying victimisation amongst first-episode psychosis patients and unaffected controls. Schizophr Res 2013, 150:169-175, doi: 10.1016/j.schres.2013.07.001

57. Varese $F$, Smeets $F$, Drukker $M$, Lieverse $R$, Lataster $T$, Viechtbauer $W$ et al. Childhood Adversities Increase the Risk of Psychosis: A Meta-analysis of Patient-Control, Prospective and Cross-sectional Cohort Studies. Schizophr Bull 2012, 38:661-671, doi: 10.1093/schbul/sbs050

58. Allen JG, Coyne L, Console DA. Dissociative detachment relates to psychotic symptoms and personality decompensation. Compr Psychiatry 1997, 38:327-334, doi: 10.1016/S0010 440X(97)90928-7

59. Bentall RP, Rowse G, Shryane N, Kinderman P, Howard R, Blackwood $\mathrm{N}$ et al. The cognitive and affective structure of paranoid delusions: a transdiagnostic investigation of patients with schizophrenia spectrum disorders and depression. Arch
Gen Psychiatry 2009, 66:236-247, doi: 10.1001/archgenpsychiatry.2009.1

60. Dong M, Anda R, Dube SR, Giles WH, Felitti VJ. The relationship of exposure to childhood sexual abuse to other forms of abuse, neglect and household dysfunction during childhood. Child Abuse Negl 2003. 27:625-639, PMID:12818611

61. Selten JP, Cantor-Graae E. Hypothesis: social defeat is a risk factor for schizophrenia? Br J Psychiatry 2007, 51:s9-s12, doi:10.1192/bjp.191.51.s9

62. Ventura J, Nuechterlein KH, Subotnik KL, Hardesty JP, Mintz $\mathrm{J}$. Life events can trigger depressive exacerbation in the early course of schizophrenia. J Abnorm Psychol 2000, 109:139-144, PMID: 1074094

63. Horan WP, Ventura J, Nuechterlein KH, Subotnik KL, Hwang SS, Mintz J. Stressful life events in recent-onsetschizophrenia: reduced frequencies and altered subjective appraisals. Schizophr Res 2005, 75:363-374, doi:10.1016/j.schres.2004.07.019

64. Myin-Germeys I, van Os J. Stress-reactivity in psychosis: evidence for an affective pathway to psychosis. Clin Psychol Rev 2007, 27:409-424, doi:10.1016/j.cpr.2006.09.005

65. Myin-Germeys I, van Os J, Schwartz JE, Stone AA, Delespaul PA. Emotional reactivity to daily life stress in psychosis. Arch Gen Psychiatry 2001, 58:1137-1144, PMID:11735842

66. Myin-Germeys I, Krabbendam L, Jolles J, Delespaul PA, van Os J. Are cognitive impairments associated with sensitivity to stress in schizophrenia? An experience sampling study. Am J Psychiatry 2002, 159:443-449, doi:10.1176/appi.ajp.159.3.443

67. Larkin W, Read J. Childhood trauma and psychosis: evidence, pathways, and implications. J Postgrad Med 2008, 54:287-293, PMID: 18953148

68. Vargas T, Lam PH, Azis M, Osborne KJ, Lieberman A, Mittal VA. Childhood Trauma and Neurocognition in Adults With Psychotic Disorders: A Systematic Review and Meta-analysis. Schizophr Bull 2018, Epub ahead of print, doi: 10.1093/schbul/ sby 150

69. Aas M, Navari S, Gibbs A, Mondelli V, Fisher HL, Morgan $C$ et al. Is there a link between childhood trauma, cognition, and amygdala and hippocampus volume in first-episode psychosis? Schizophr Res 2012, 137:73-79, doi: 10.1016/j. schres.2012.01.035

70. Alameda L, Golay P, Baumann PS, Progin P, Mebdouhi N, Elowe $\mathrm{J}$ et al. Mild Depressive Symptoms Mediate the Impact of Childhood Trauma on LongTerm Functional Outcome in Early Psychosis Patients. Schizophr Bull 2017, 43:1027-1035, doi: 10.1093/schbul/sbw163

71. Chatziioannidis S, Andreou Ch, Agorastos A, Kaprinis S, Malliaris Y, Garyfallos G, Bozikas VP. The role of attachment anxiety in the relationship between childhood trauma and schizophreniaspectrum psychosis Psychiatry Res 2019, 276:223-231, doi: 10.1016/j.psychres.2019.05.021.

72. Konings M, Stefanis NC, Kuepper R, de Graaf R, ten Have $\mathrm{M}$, van Os $\mathrm{J}$ et al. Replication in two independent populationbased samples that childhood maltreatment and cannabis use synergistically impact on psychosis risk. Phychol Med 2012, 42:149-159, doi: 10.1017/S0033291711000973 
73. Morgan C, Reininghaus U, Reichenberg A, Frissa S, SELCoH study team, Hotopf $M$ et al. Adversity, cannabis use and psychotic experiences: evidence of cumulative and synergistic effects. Br J Psychiatry 2014, 204:346-353, doi: 10.1192/bjp. bp. 113.134452

74. Trotta A, lyegbe C, DiForti M, Sham PC, Campbell DD, Cherny SS et al. Interplay between Schizophrenia Polygenic Risk Score and Childhood Adversity in First Presentation Psychotic Disorder: A Pilot Study. PLOS ONE 2016, 11:e0163319, doi:10.1371/journal.pone.0163319

75. Guloksuz S, Pries LK, Delespaul P, Kenis G, Luykx JJ, Lin BD et al Examining the independent and joint effects of molecular genetic liability and environmental exposures in schizophrenia: results from the EUGEI study. World Psychiatry 2019, 18:173182, doi: 10.1002/wps.20629

76. Van Winkel R, Stefanis NC, Myin-Germeys I. Psychosocial Stress and Psychosis. A Review of Neurobiological Mechanisms and the Evidence of Gene-Stress Interaction. Schizophr Bull 2008 34:1095-1105, doi: 10.1093/schbul/sbn101
77. Heinz A, Schlagenhauf F. Dopaminergic dysfunction in schizophrenia: salience attribution revisited. Schizophr Bull 2010, 36:472-485, doi: 10.1093/schbul/sbq031

78. Murray GK. The emerging biology of delusions. Psychol Med 2011, 41:7-13, doi: 10.1017/S0033291710000413

79. Murray RM. Mistakes I Have Made in My Research Career. Schizophr Bull 2017, 43:253-256, doi: 10.1093/schbul/sbw165

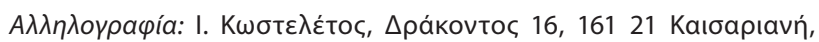

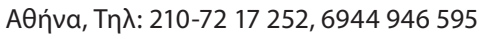

e-mail: jiankost@gmail.com 


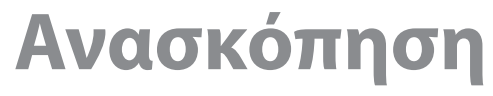 Review
}

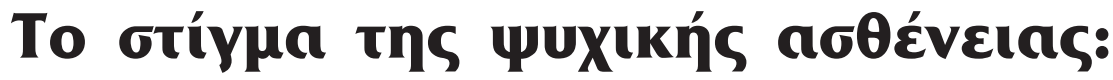

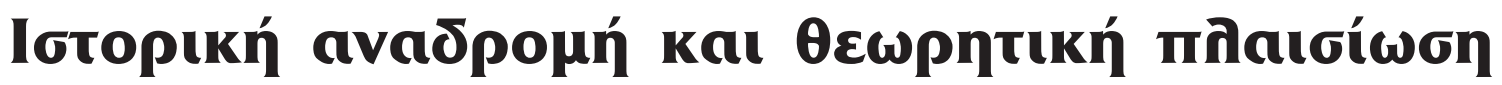

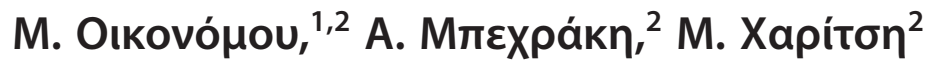

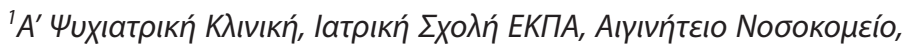

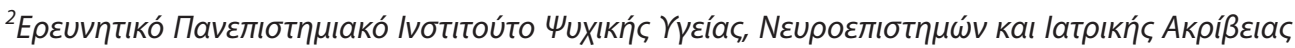

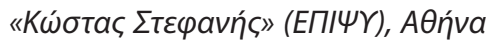

Uuxıатрıкń 2020, 31:36-46

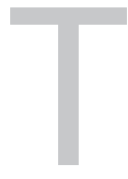

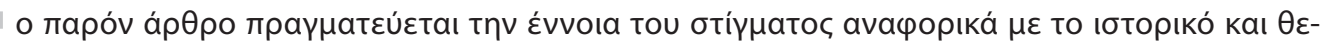

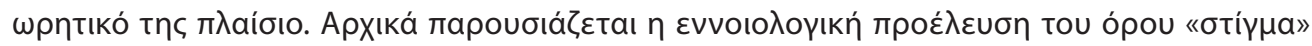

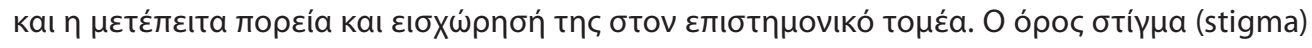

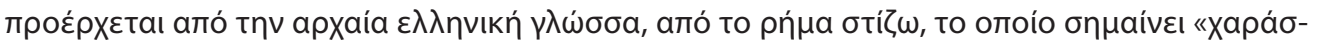

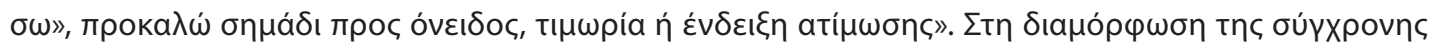

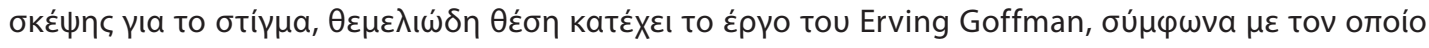

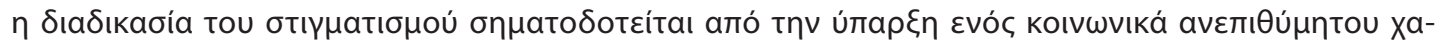

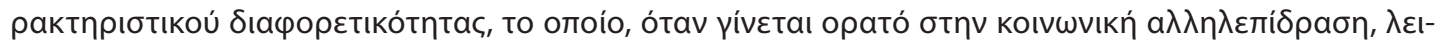

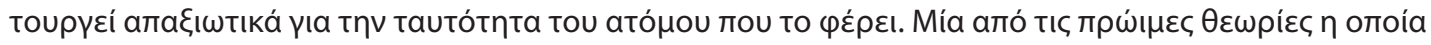

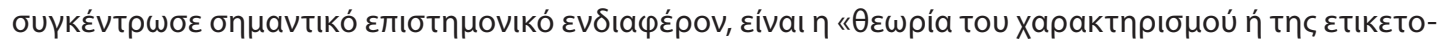

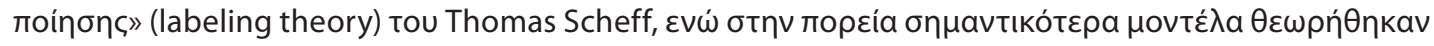

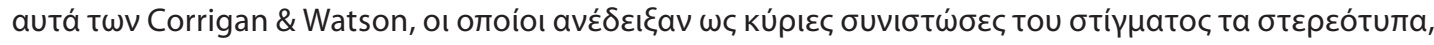

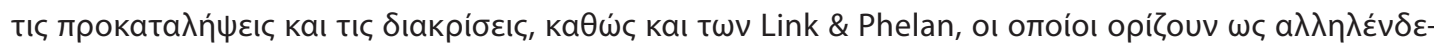

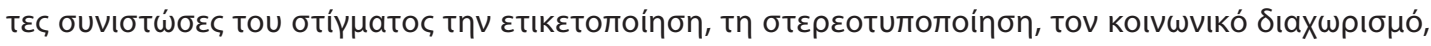

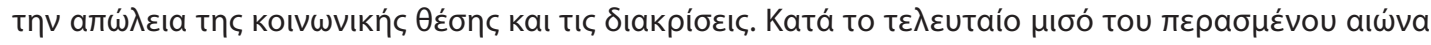

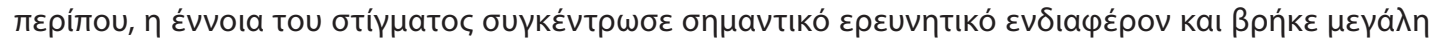

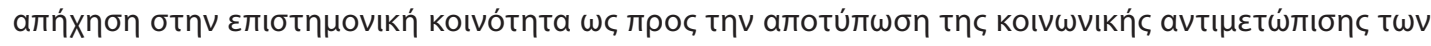

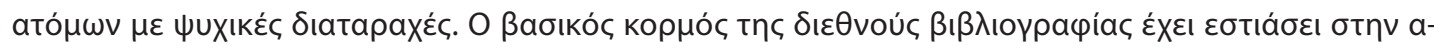

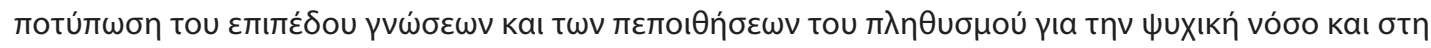

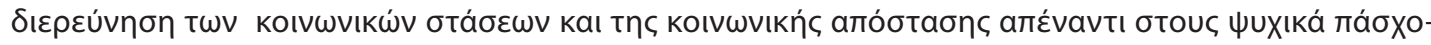

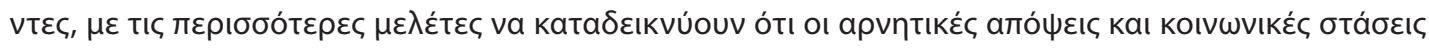




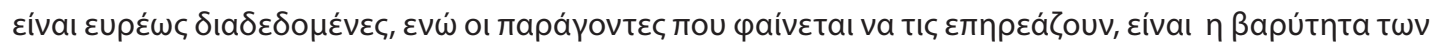

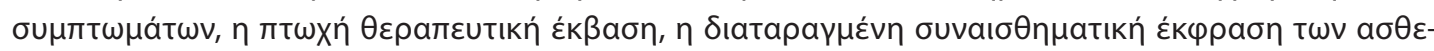

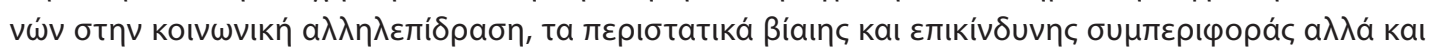

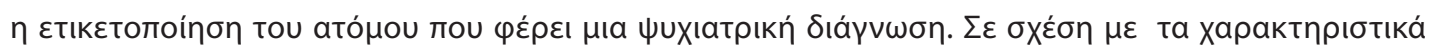

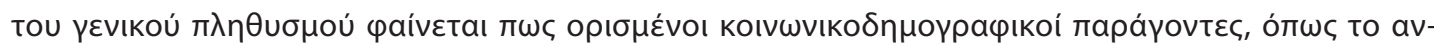

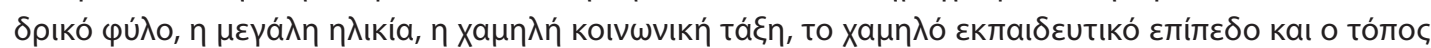

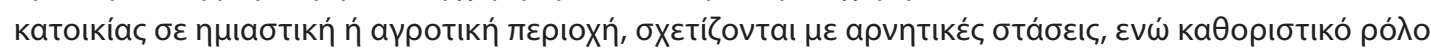

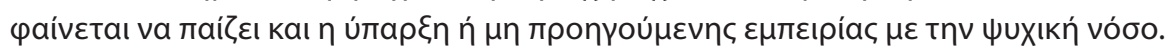

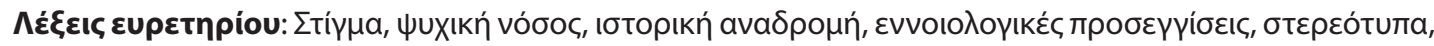

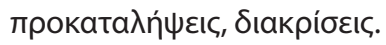

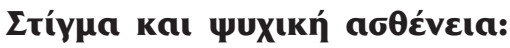

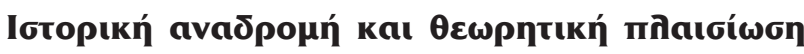

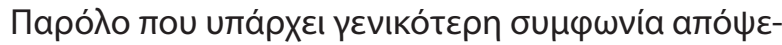

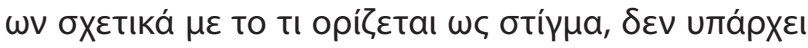

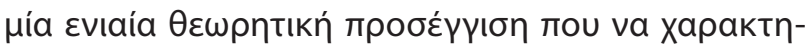

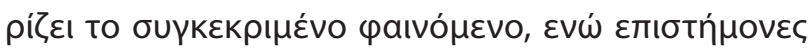

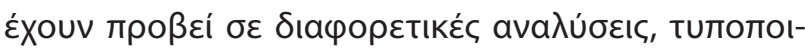

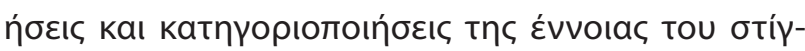

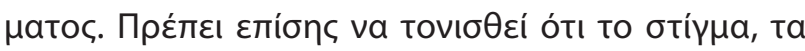

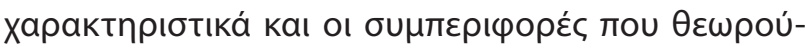

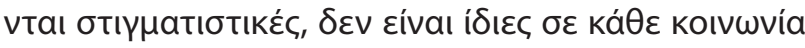

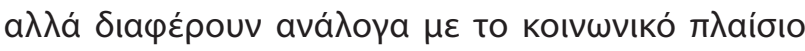

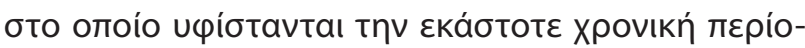

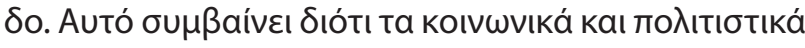

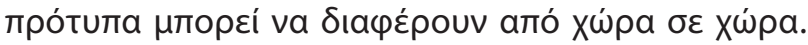

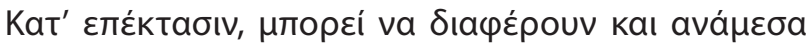

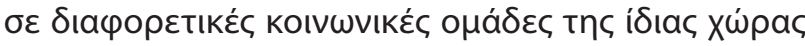

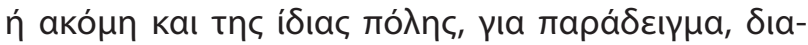

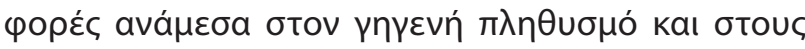

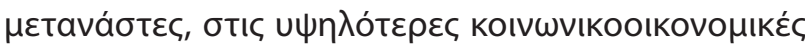

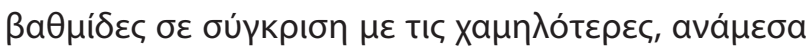

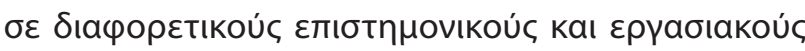

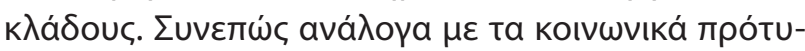

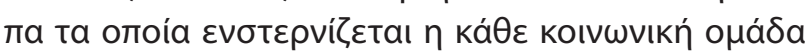

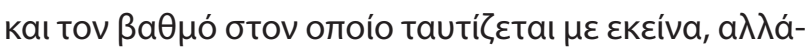

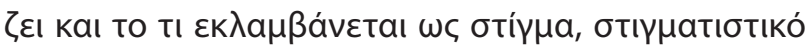

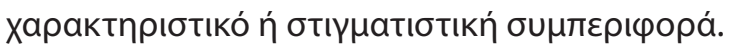

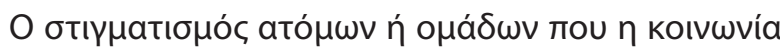

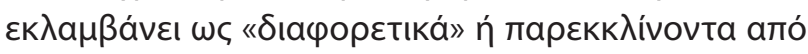

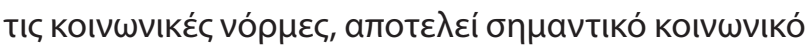

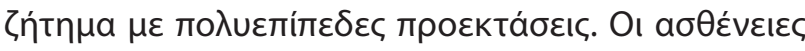

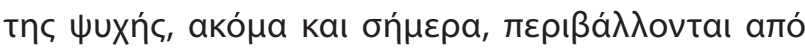

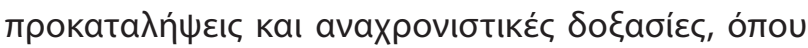

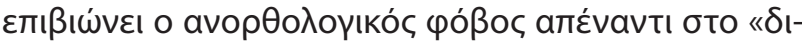

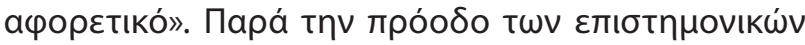

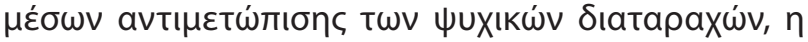

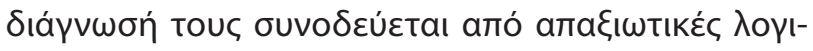

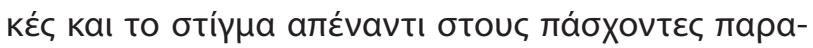

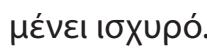

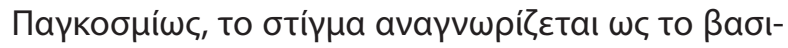

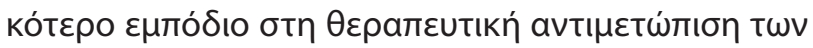

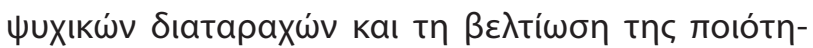

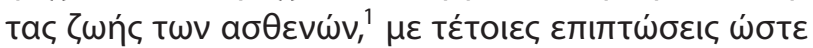

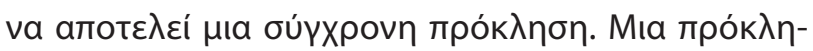

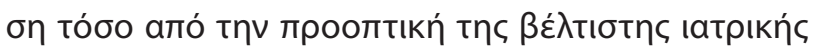

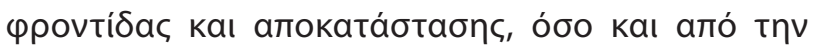

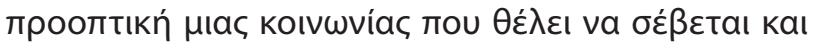

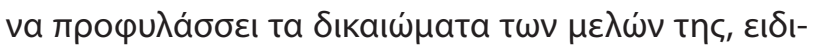

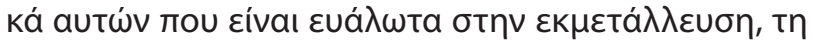

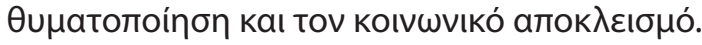

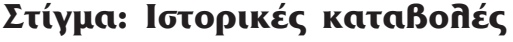

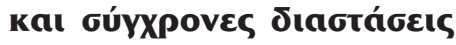

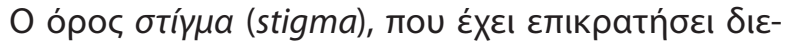

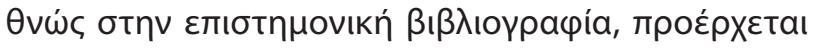

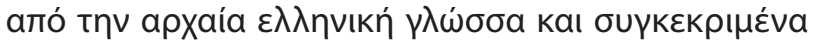

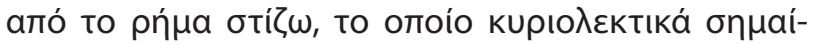

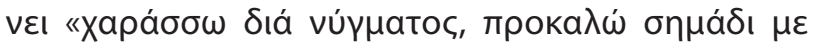

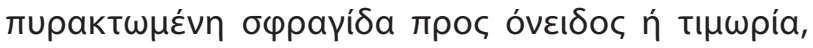

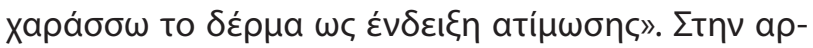

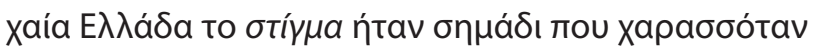

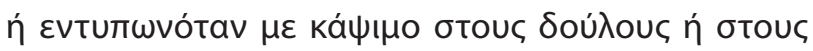

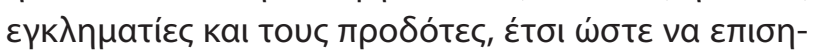

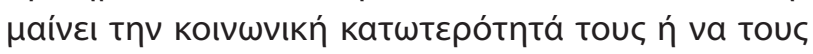

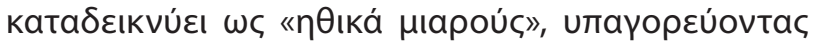

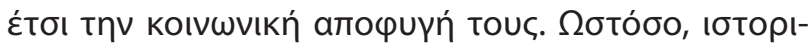

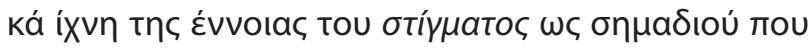

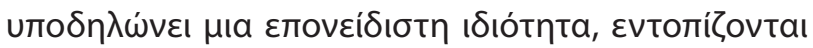

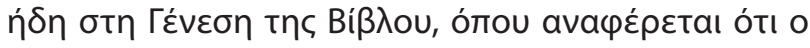

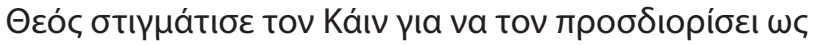




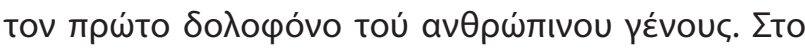

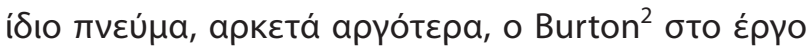

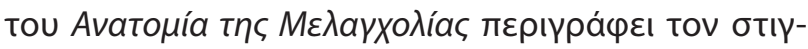

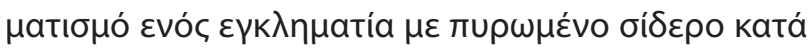

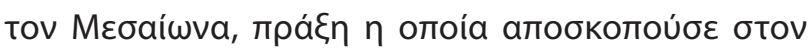

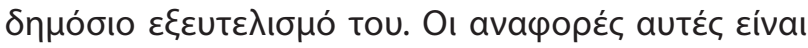

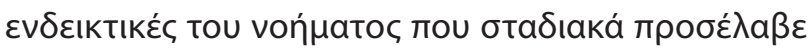

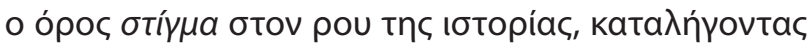

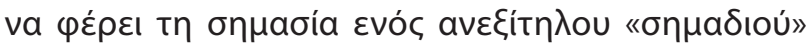

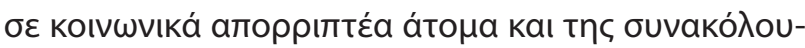

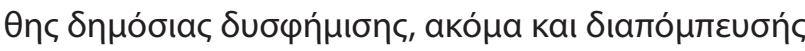
Tous.

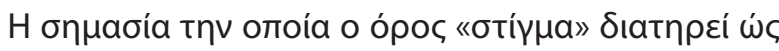

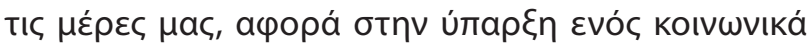

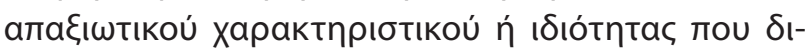

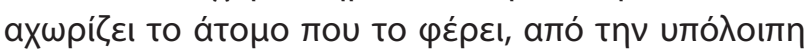

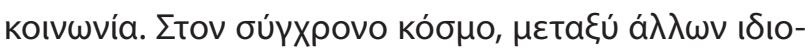

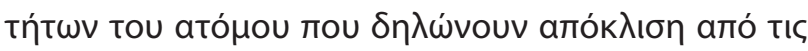

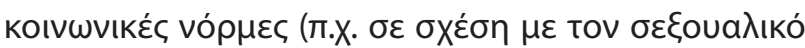

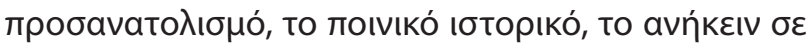

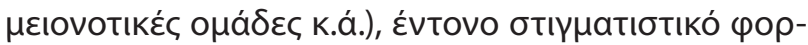

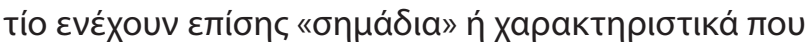

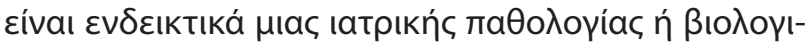

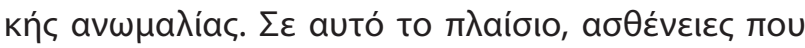

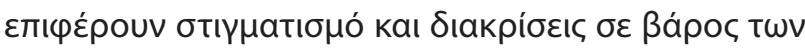

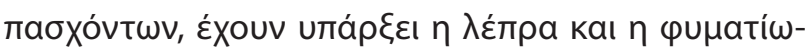

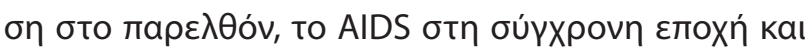

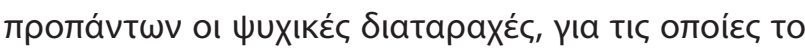

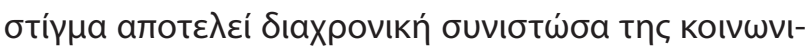

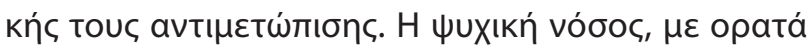

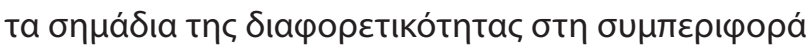

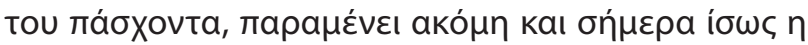

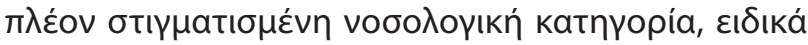

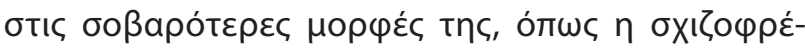
vela. ${ }^{3,4}$

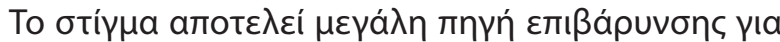

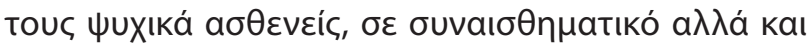

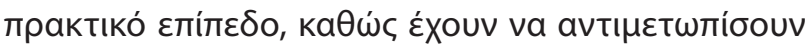

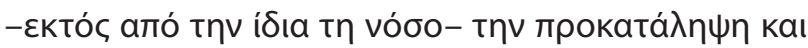

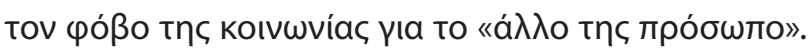

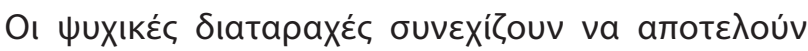

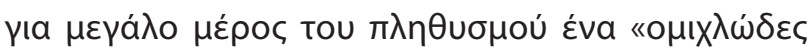

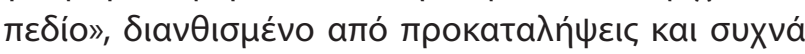

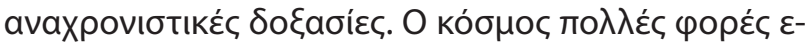

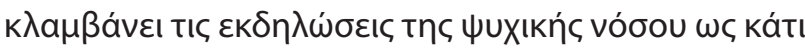

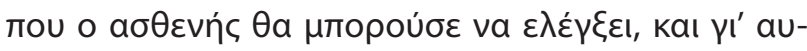

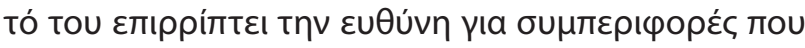

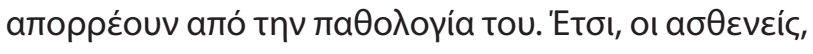

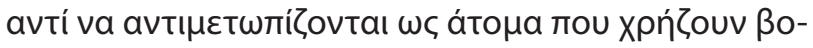

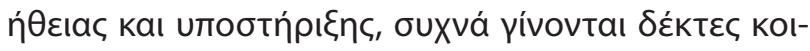

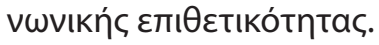

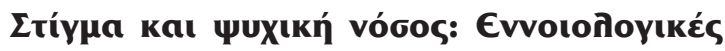

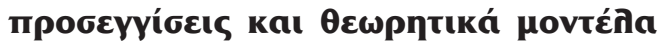

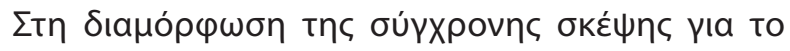

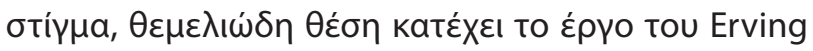

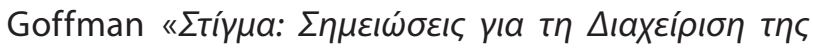

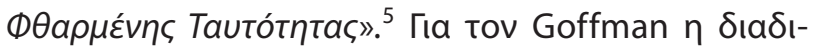

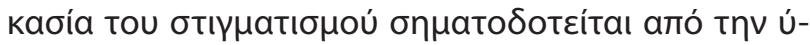

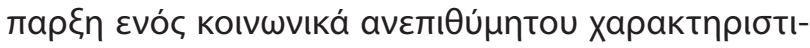

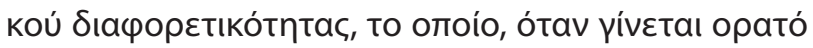

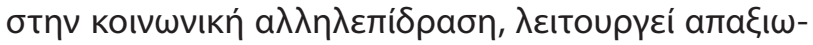

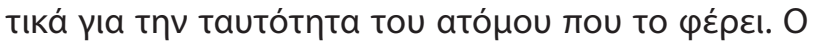

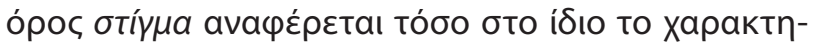

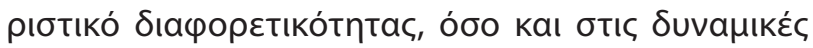

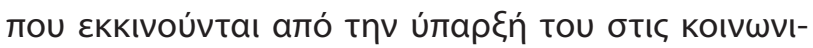

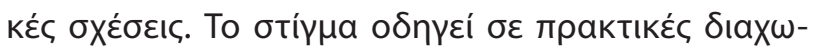

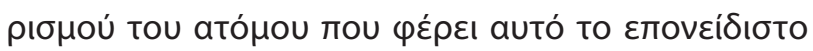

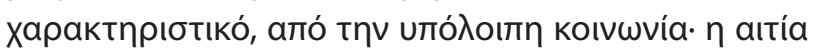

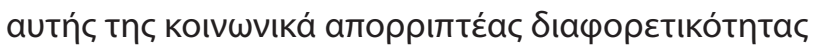

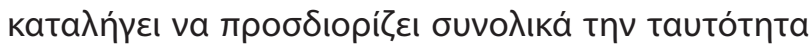

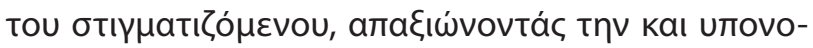

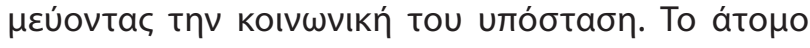

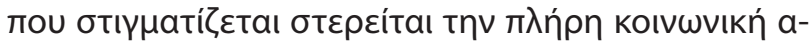

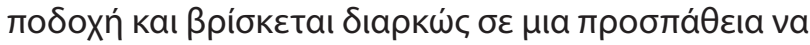

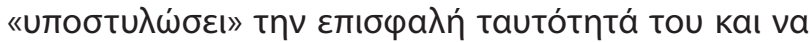

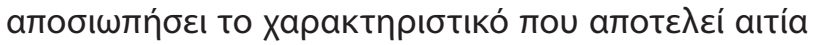

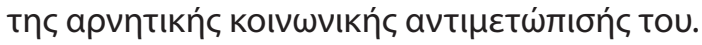

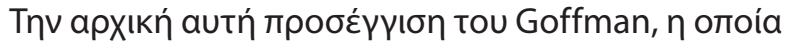

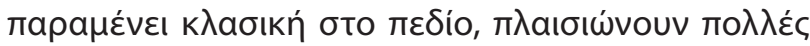

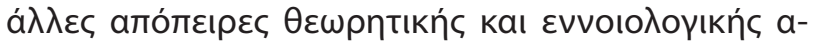

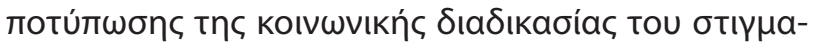

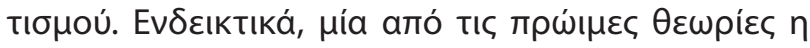

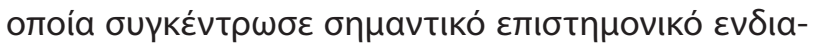

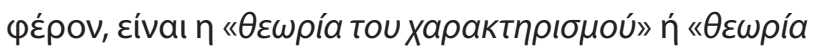

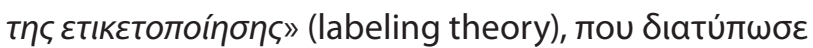
o Thomas Scheff. ${ }^{6} \Sigma u ́ \mu \varphi \omega v a \mu \varepsilon$ tn $\theta \varepsilon \omega \rho i ́ a$ autń, $\eta$

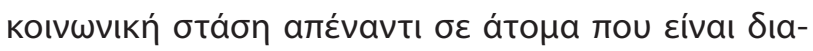

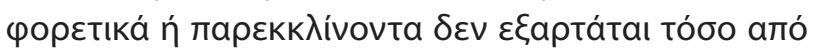

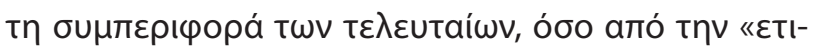

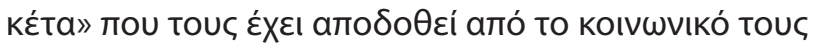

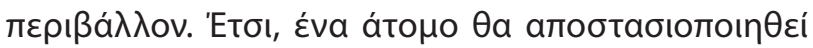

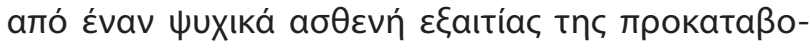

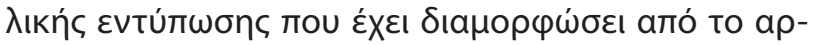




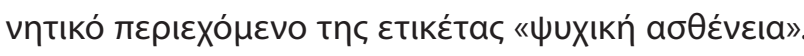

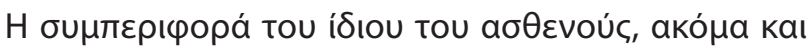

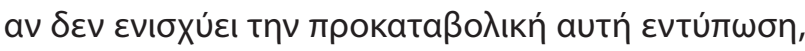

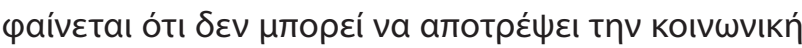

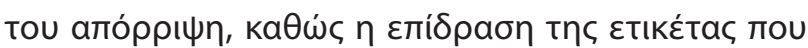

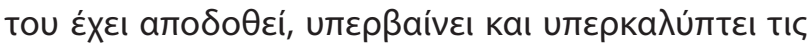

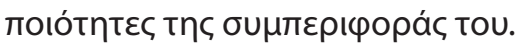

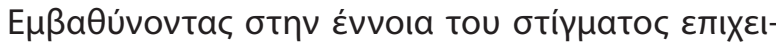

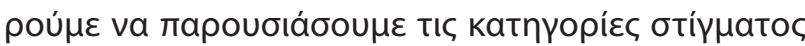

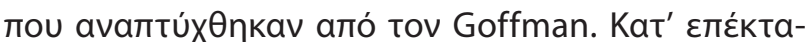

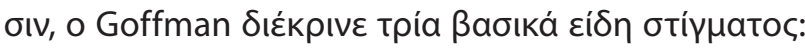

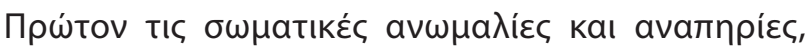

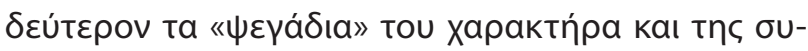

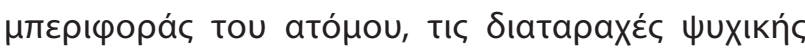

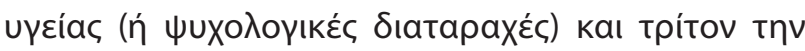

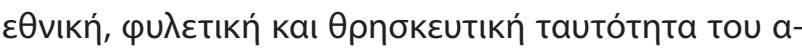

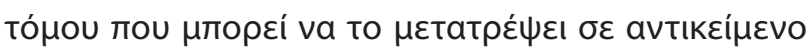

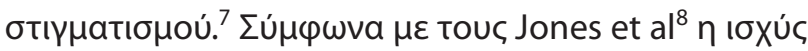

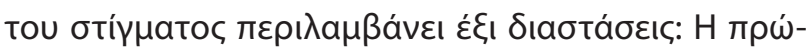

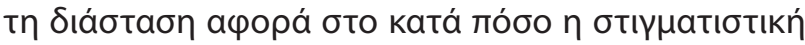

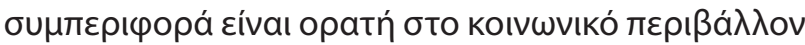

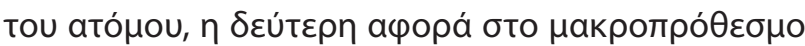

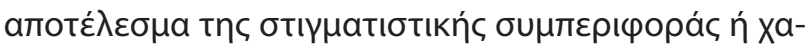

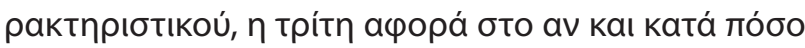

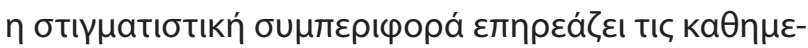

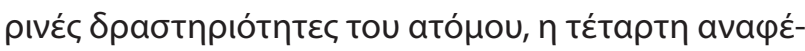

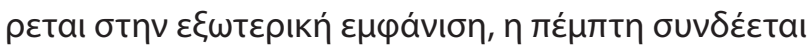

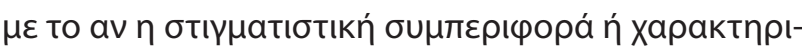

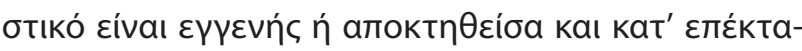

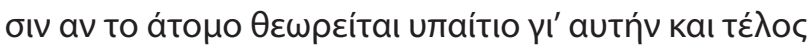

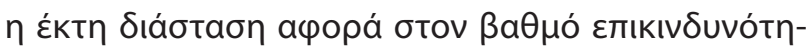

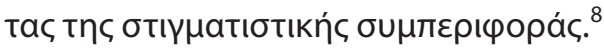

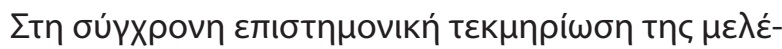

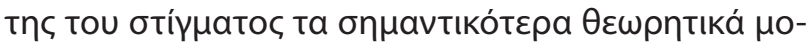

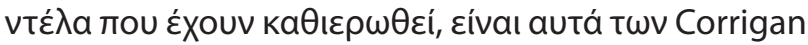
\& Watson ${ }^{9}$ kal $T \omega v$ Link \& Phelan. ${ }^{10}$ Oı Corrigan et

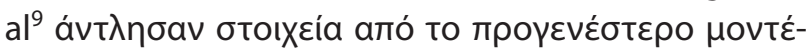

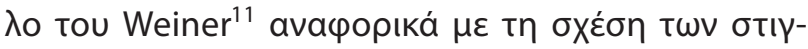

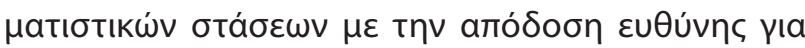

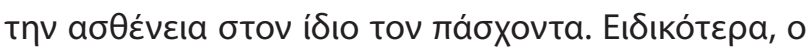

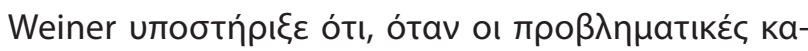

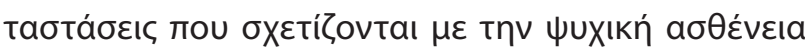

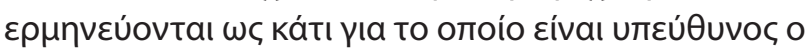

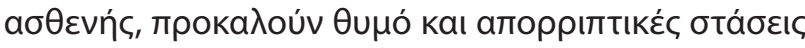

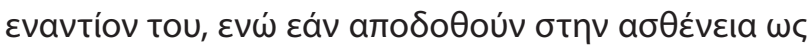

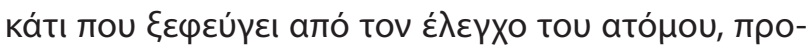

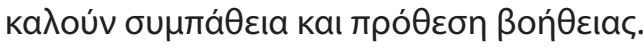

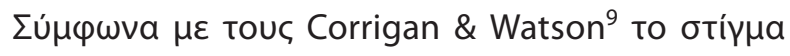

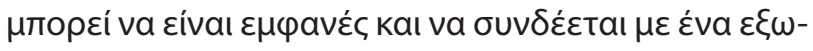

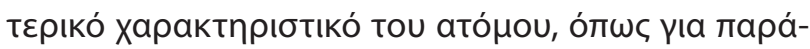

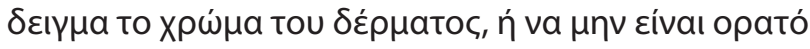

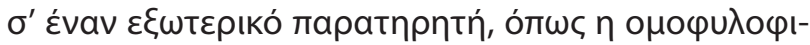

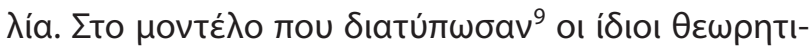

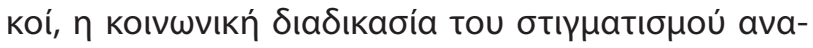

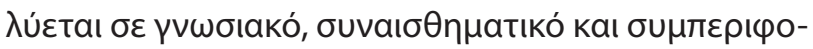

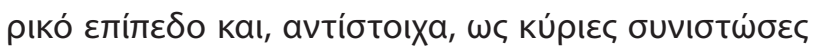

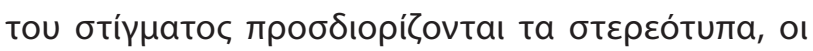

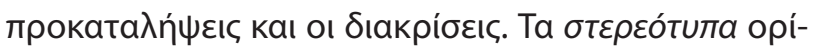

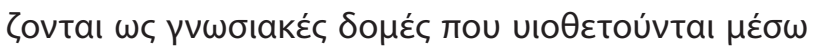

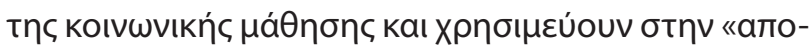

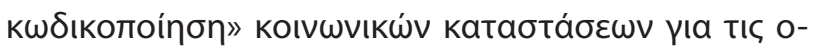

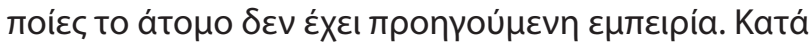

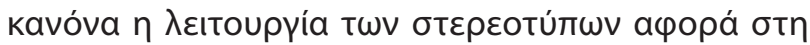

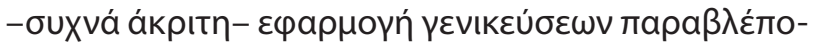

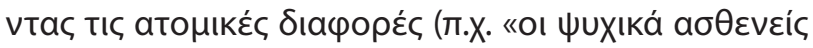

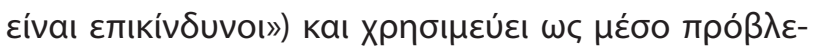

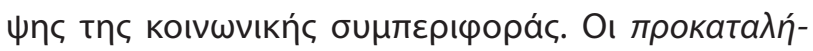

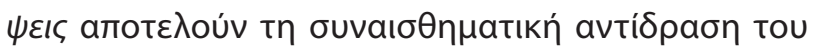

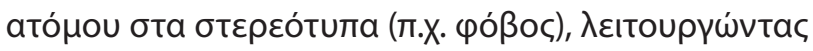

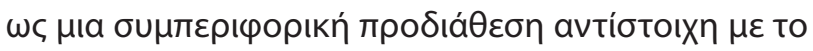

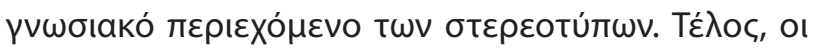

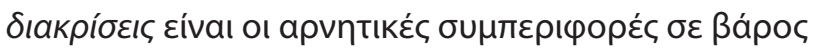

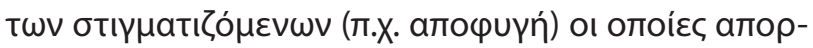

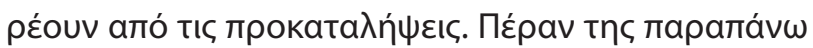

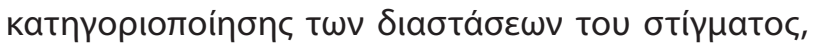

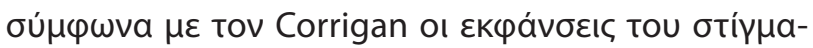

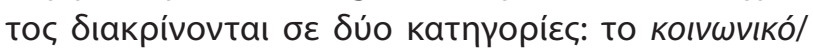

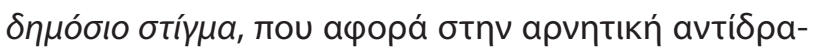

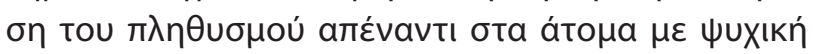

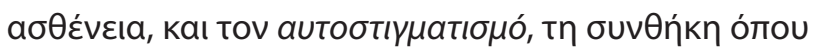

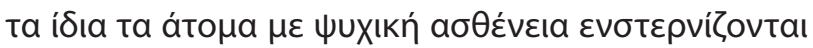

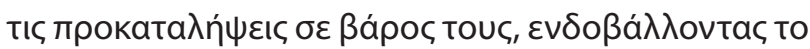

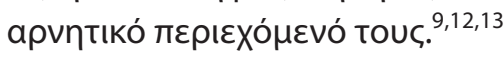

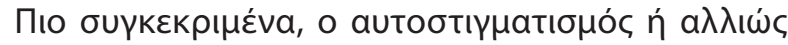

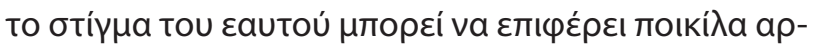

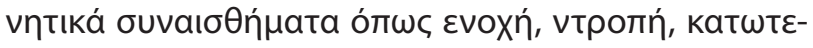

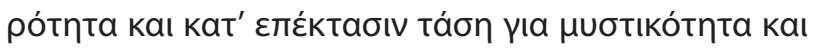

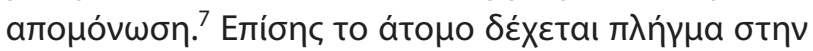

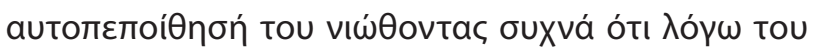

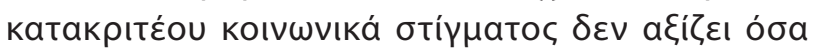

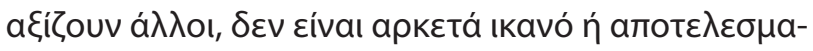

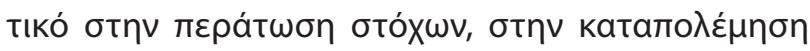

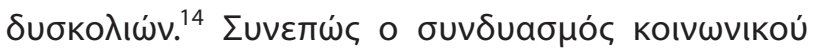

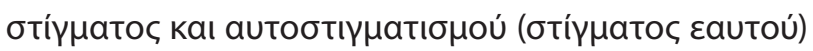




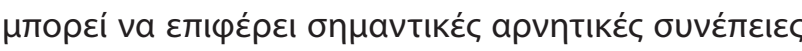

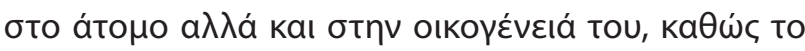

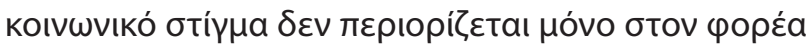

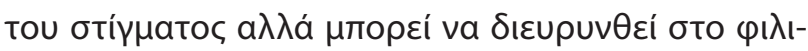

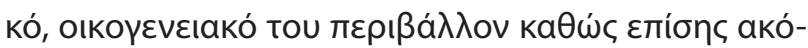

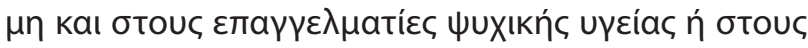

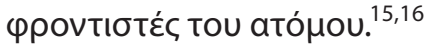

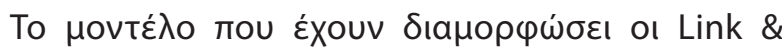

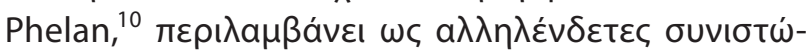

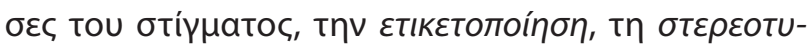

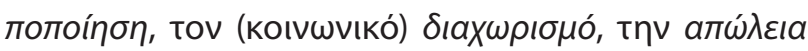

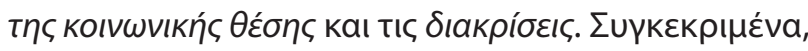

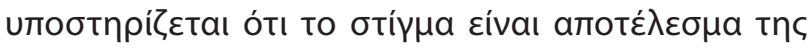

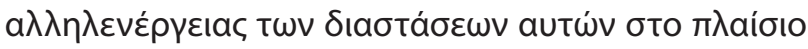

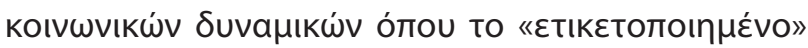

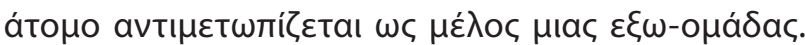

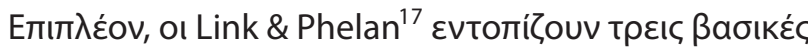

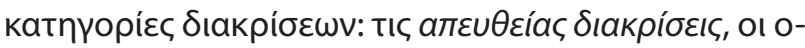

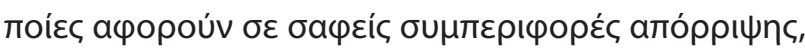

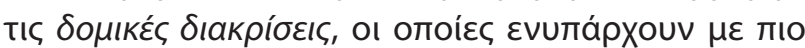

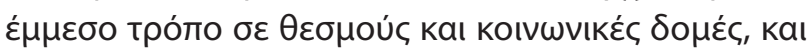

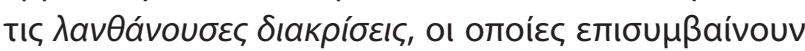

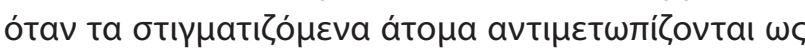

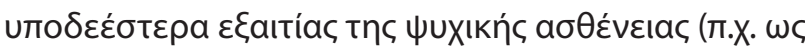

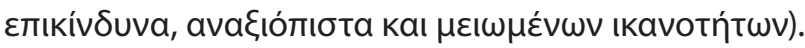

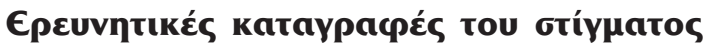

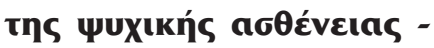

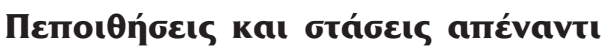

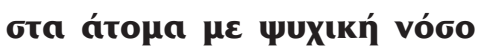

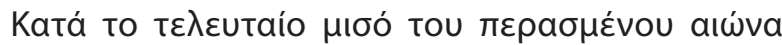

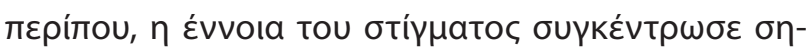

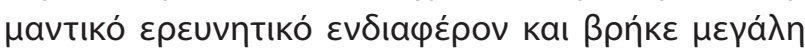
a

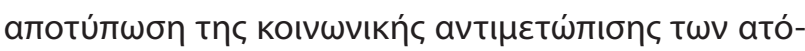

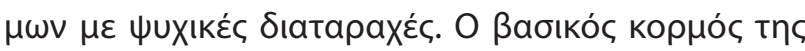

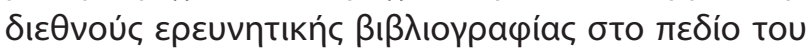

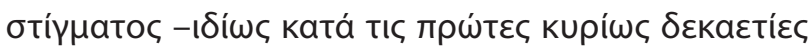

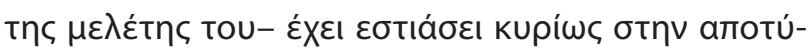

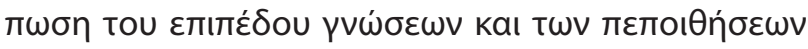

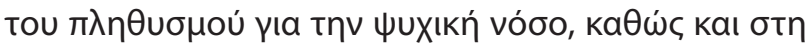

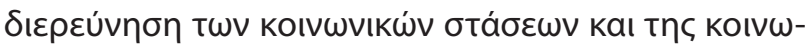

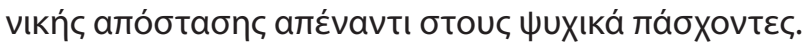

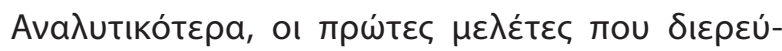

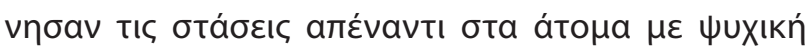

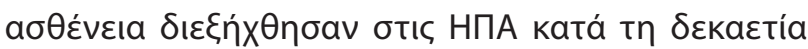

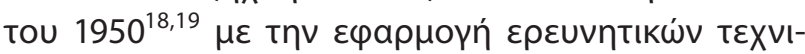

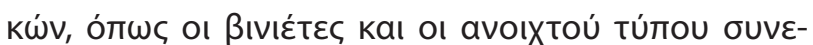

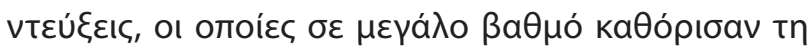

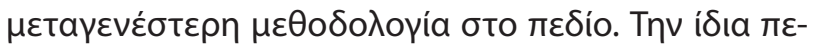

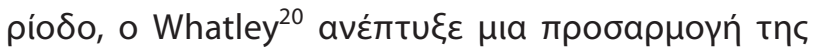

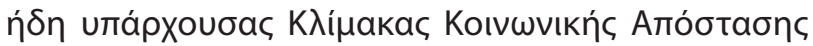

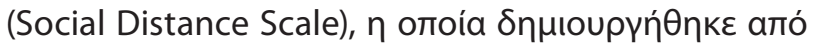

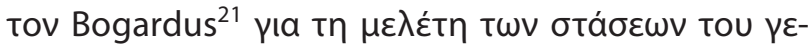

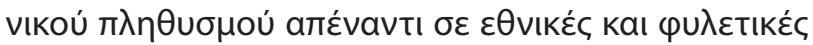

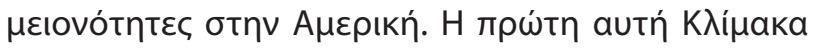

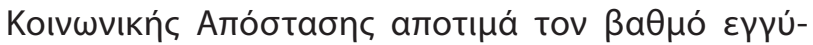

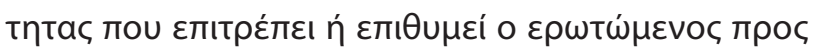

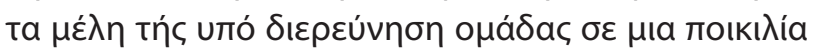

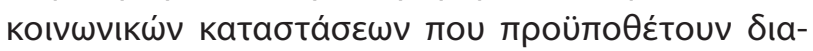

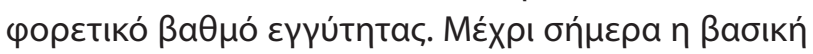

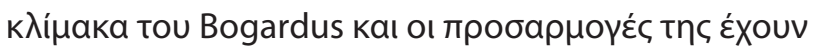

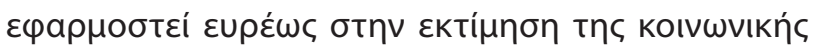

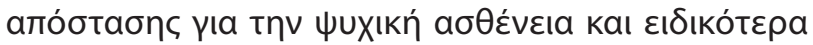

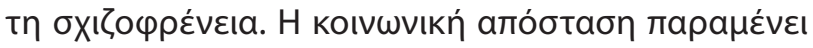

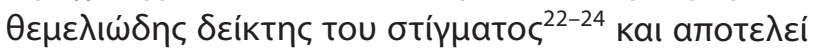

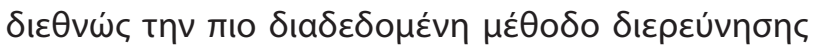

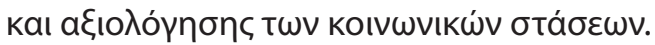

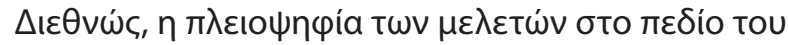

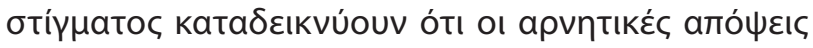

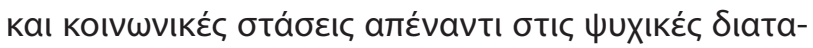

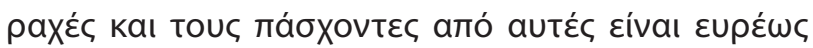

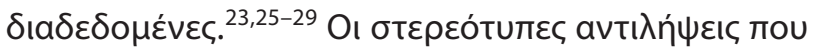

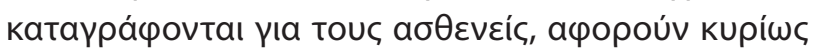

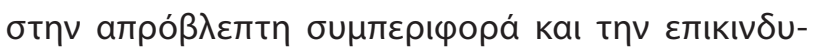

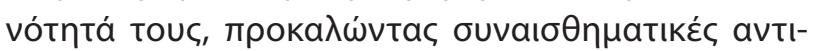

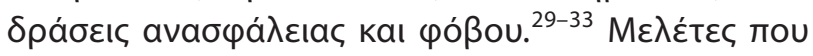

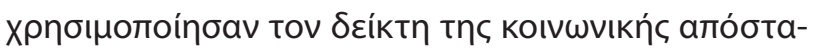

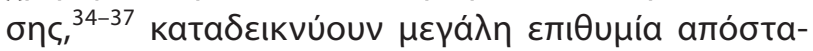

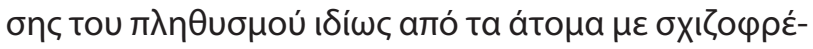

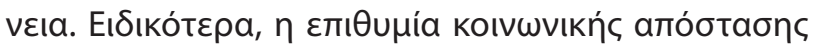

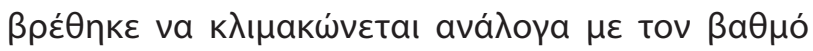

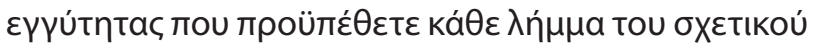

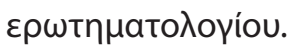

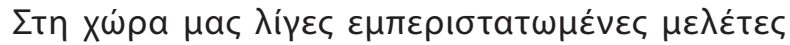

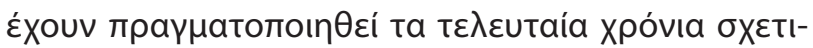

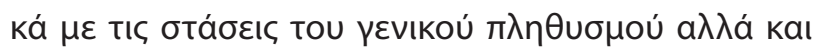

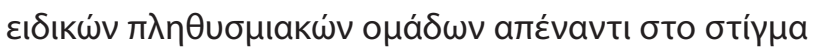

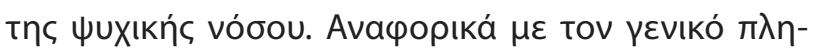

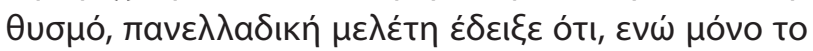

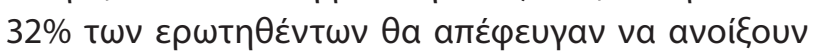

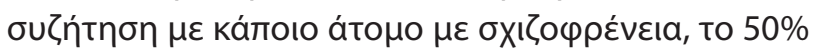

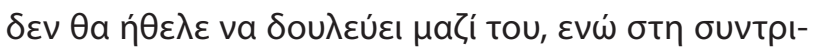

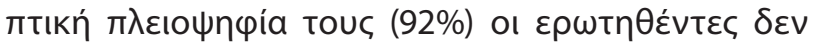




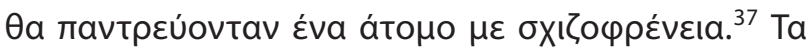

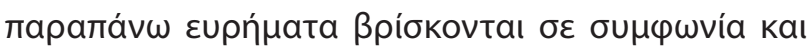

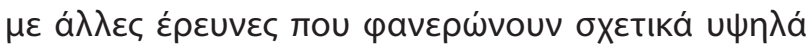

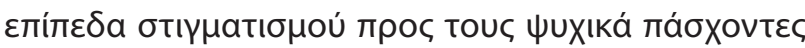

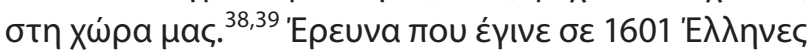

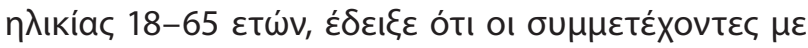

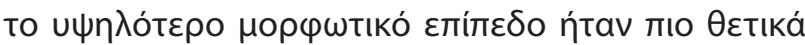

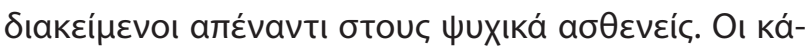

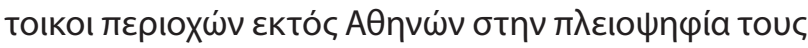

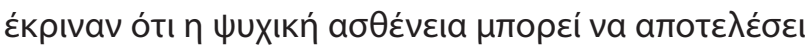

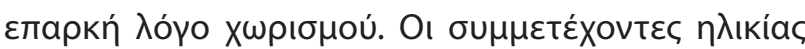

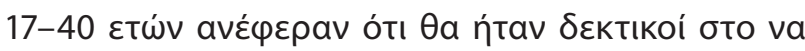

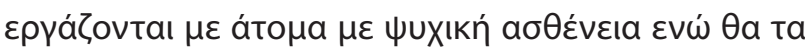

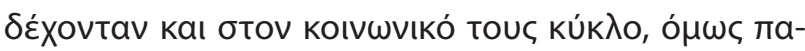

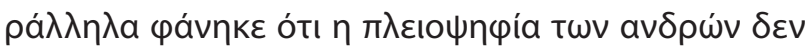

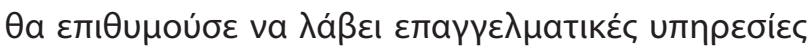

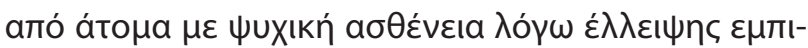

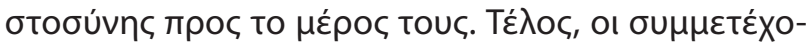

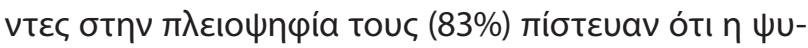

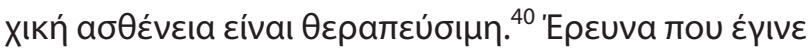

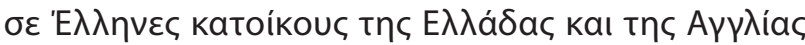

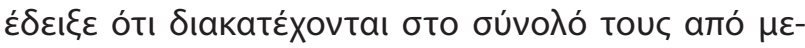

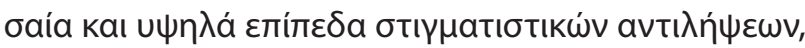

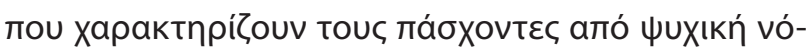

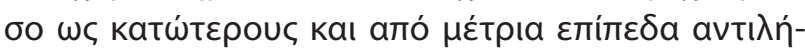

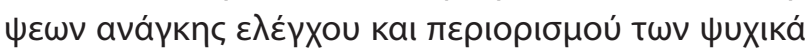

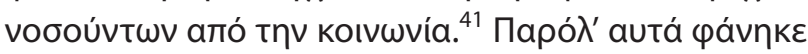

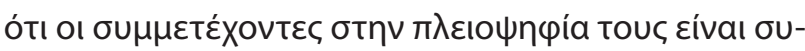

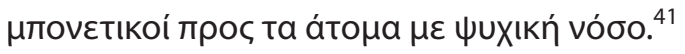

'Oбov a

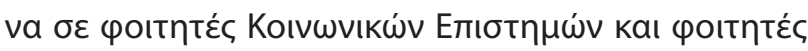

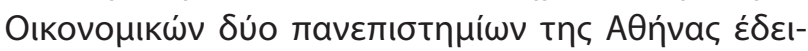

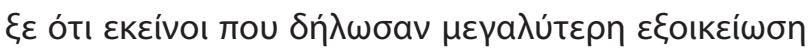

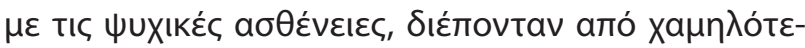

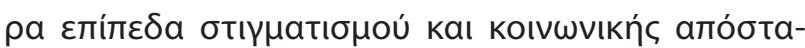

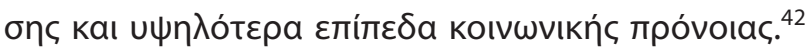

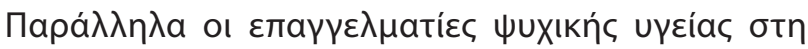

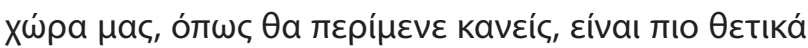

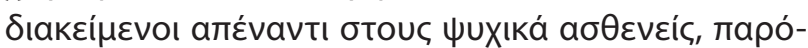

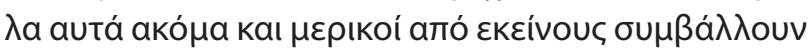

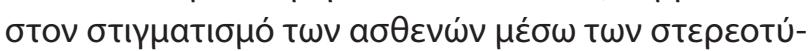

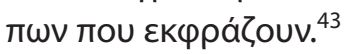

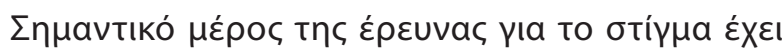

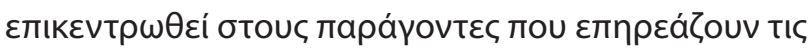

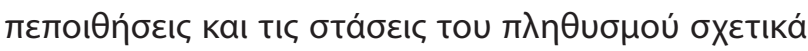

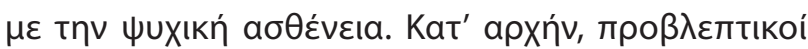

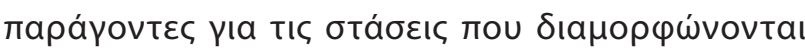

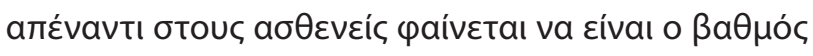

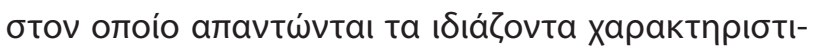

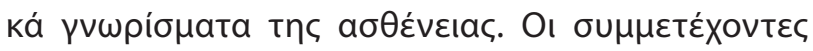

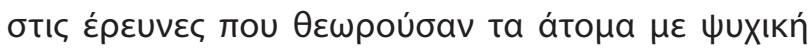

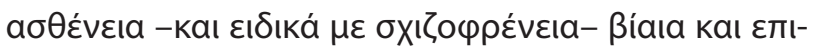

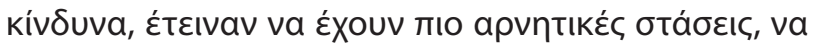

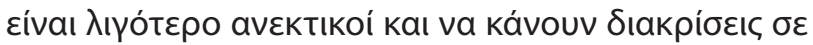

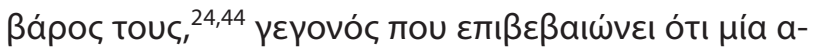

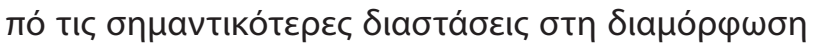

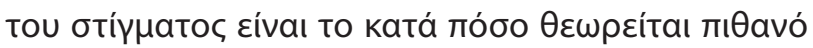

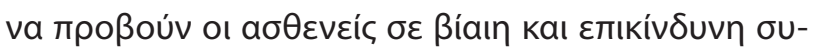
нтвріфора́.

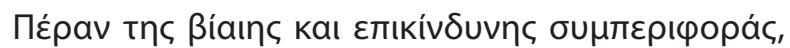

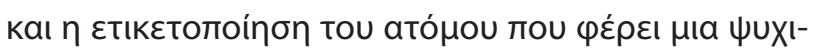

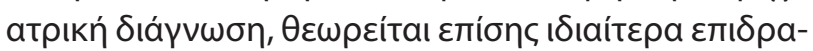

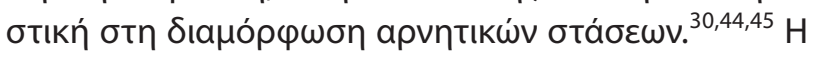

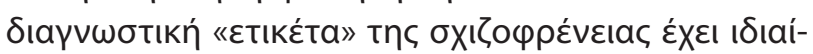

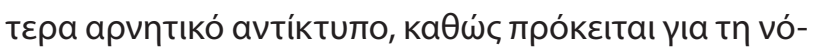

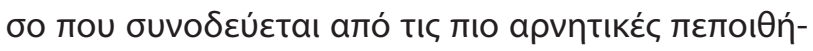

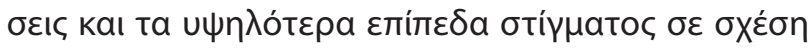

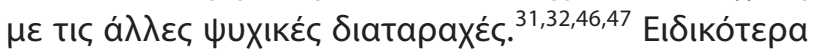

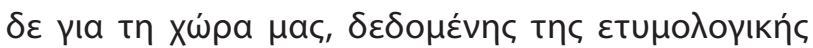

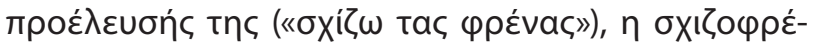

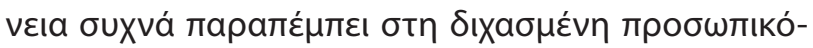

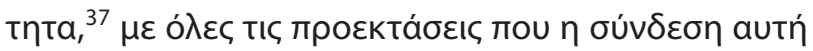

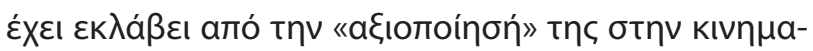

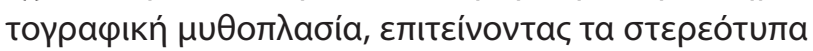

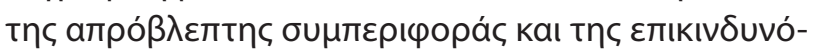

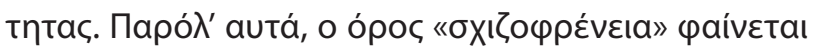

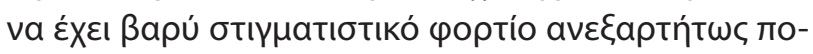

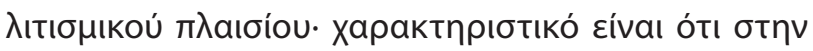

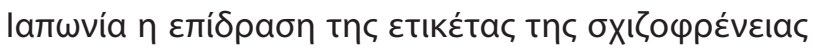

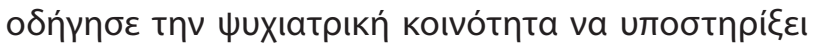

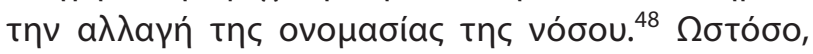

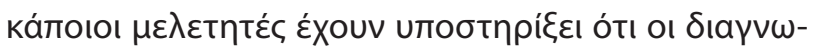

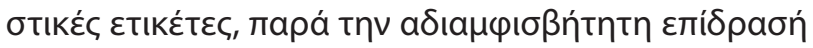

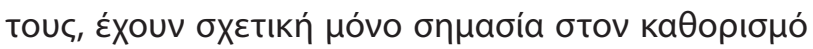

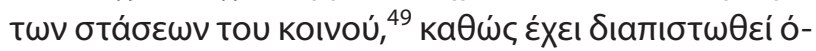

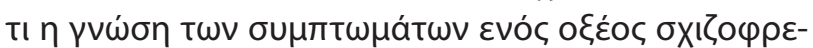

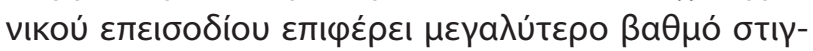

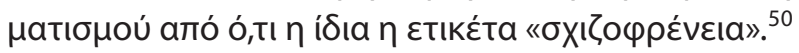

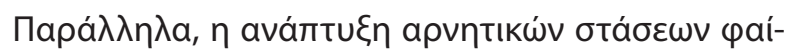

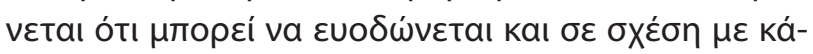

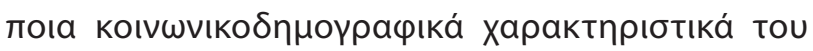

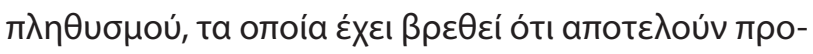

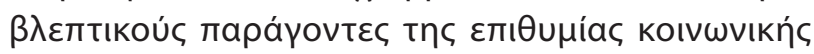

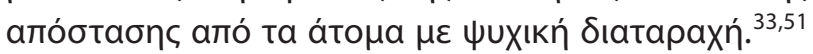




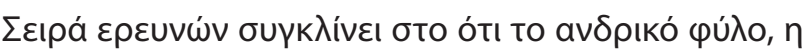

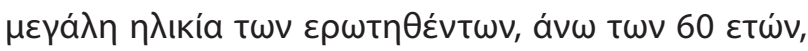

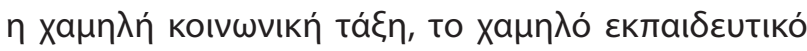

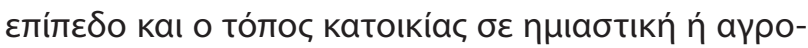

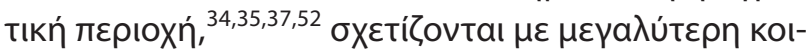

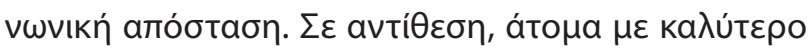

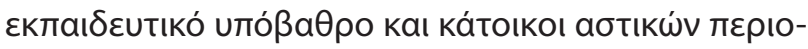

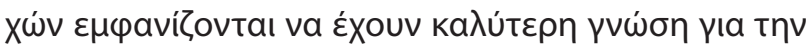

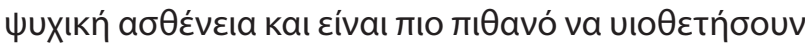

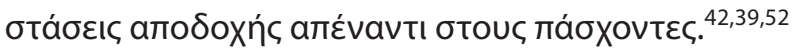

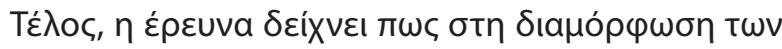

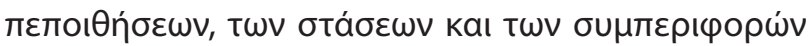

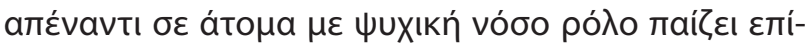

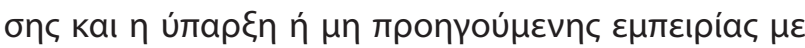

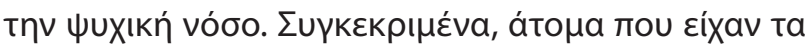

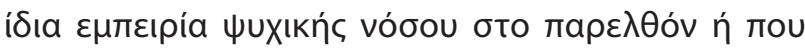

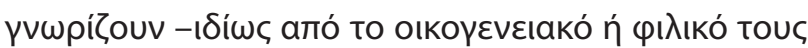

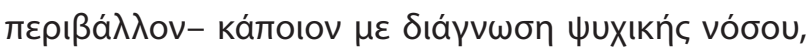

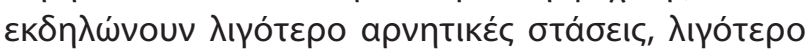

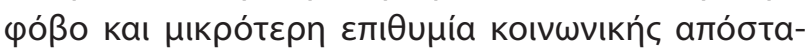

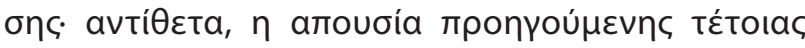

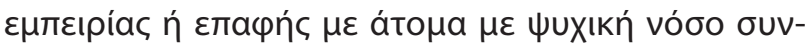

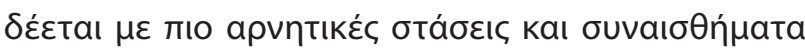

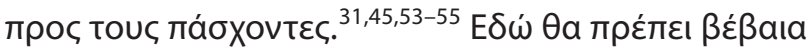

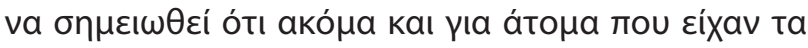

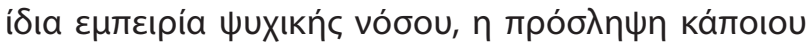

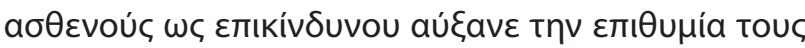

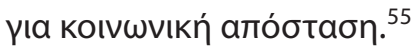

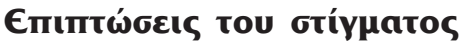

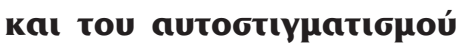

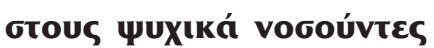

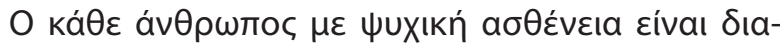

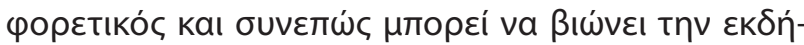

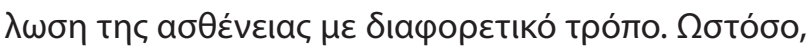

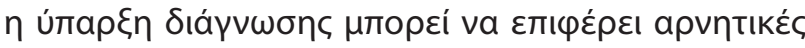

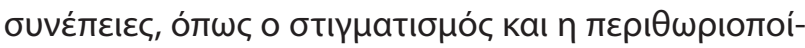

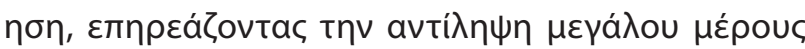

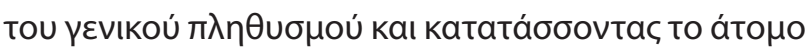

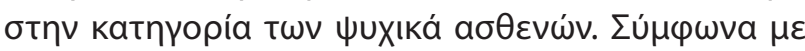

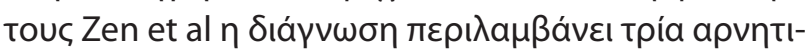

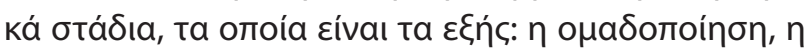

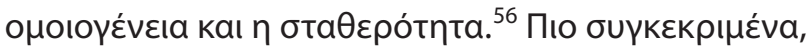

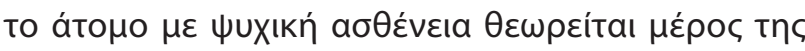

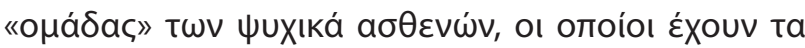

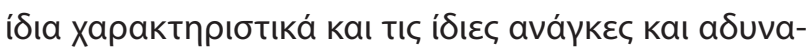

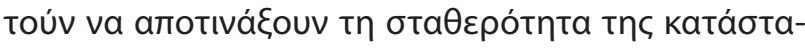

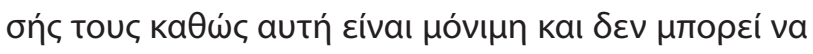

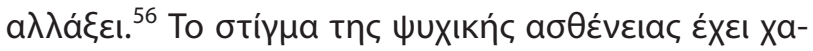

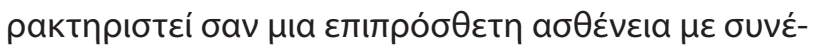

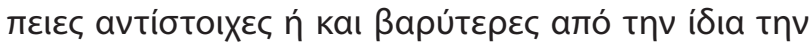

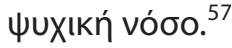

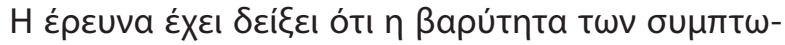

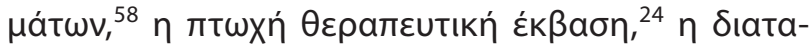

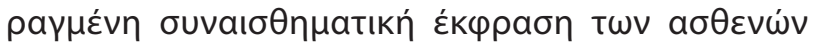

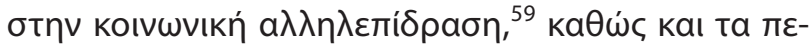

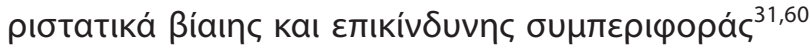

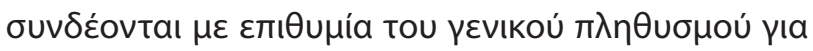

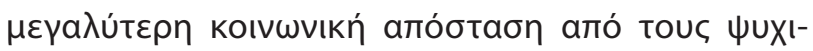

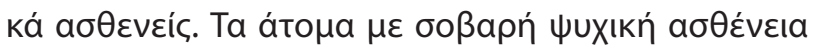

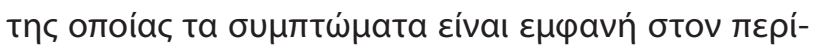

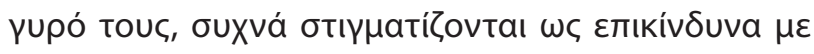

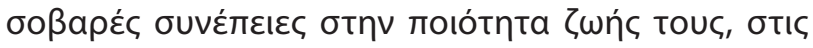

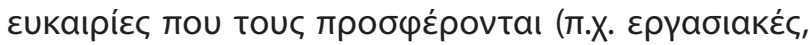

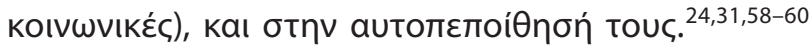

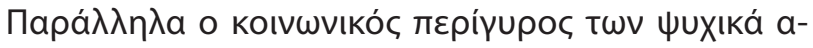

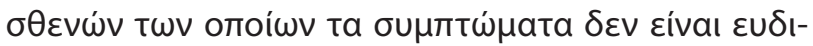

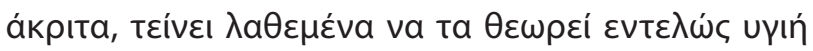

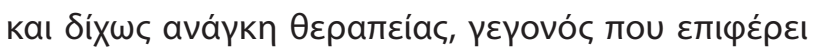

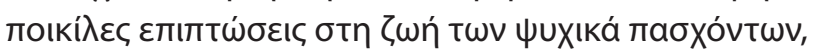

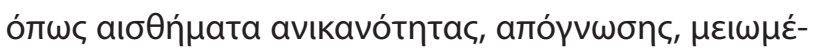

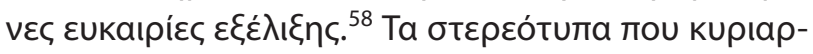

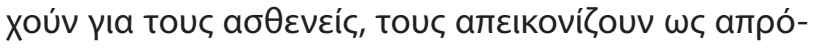

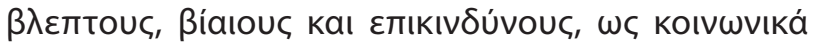

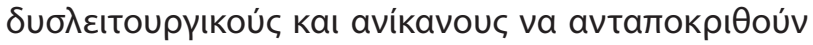

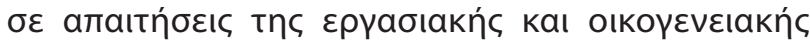

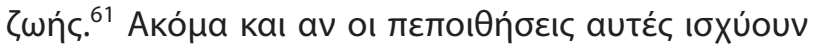

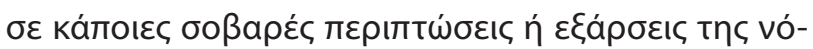

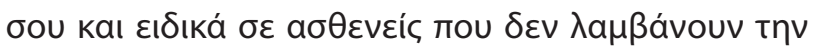

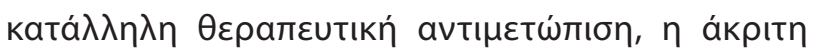

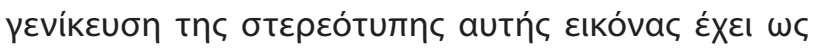

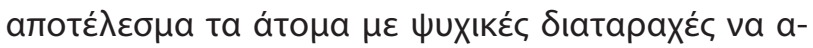

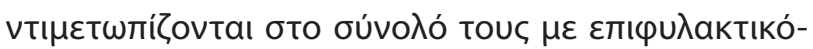

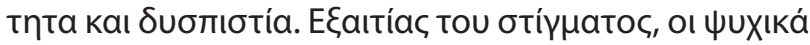

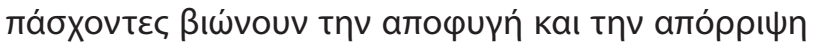

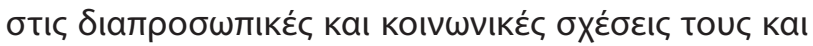

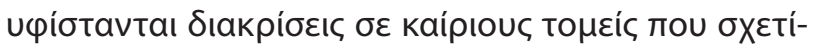

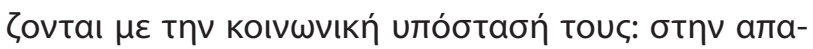

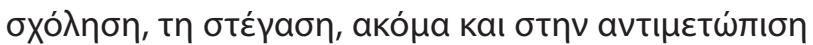

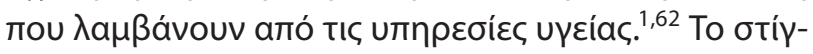

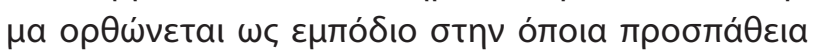

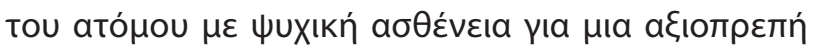

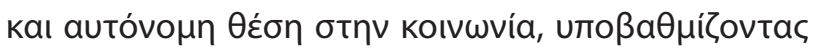

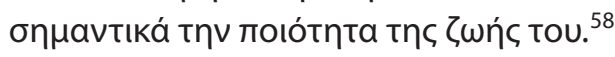




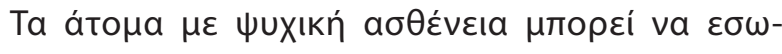

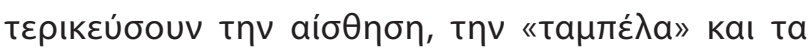

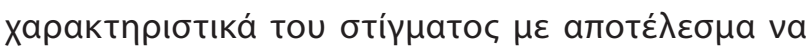

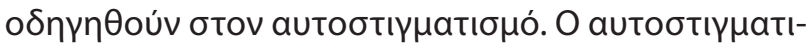

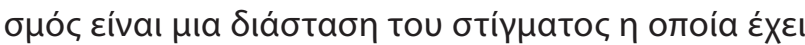

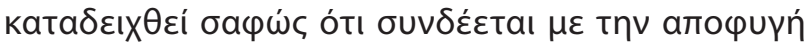

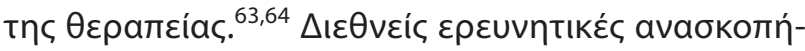

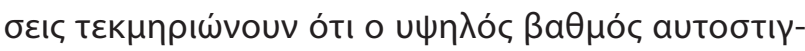

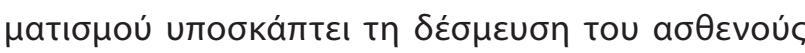

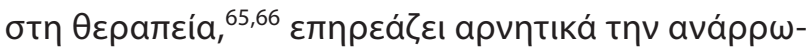

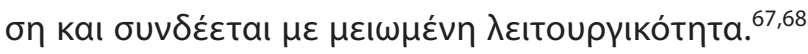

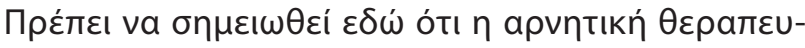

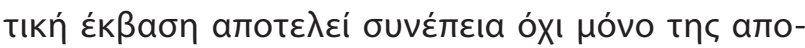

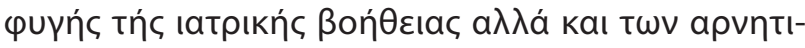

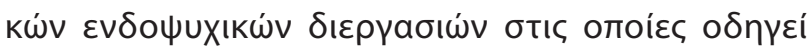

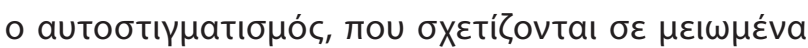

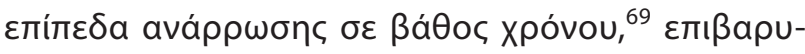

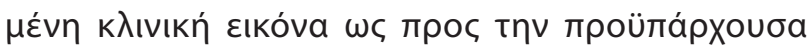

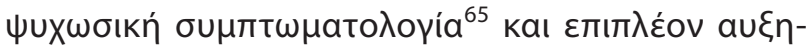

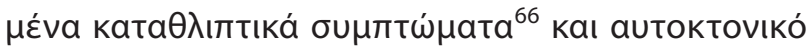

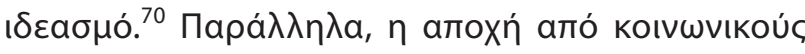

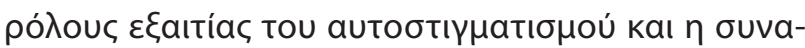

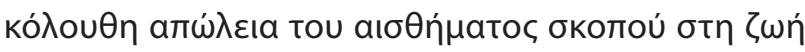

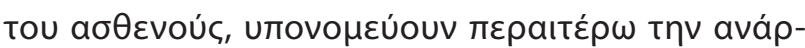
$\rho \omega \sigma n ́$ tou. ${ }^{71,72}$

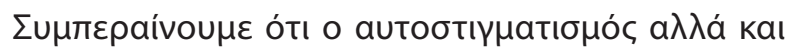

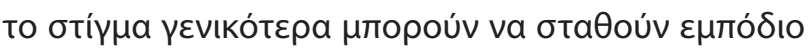

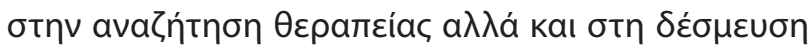

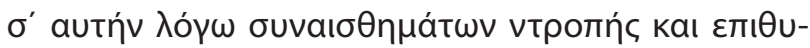

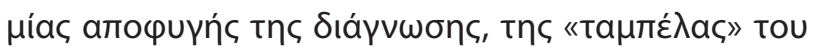

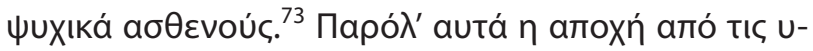

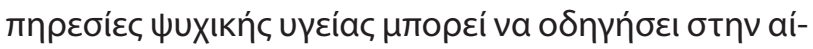

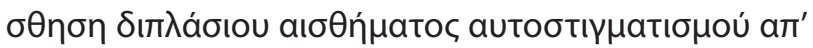

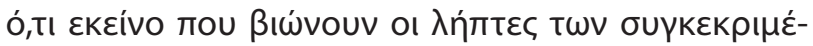

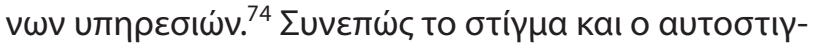

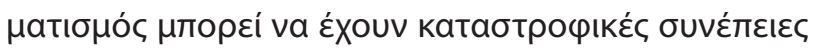

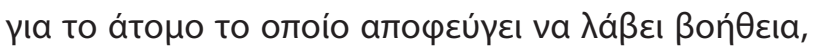

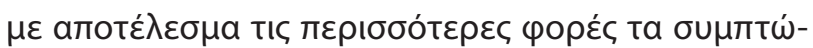

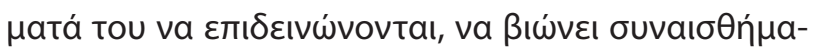

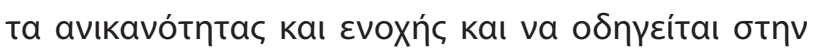

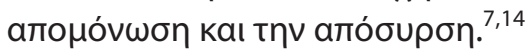

\section{Avtí ยmเกóyou}

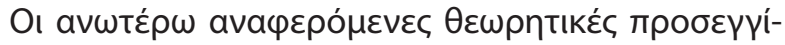

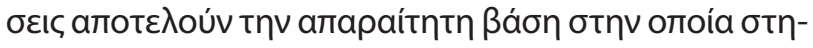

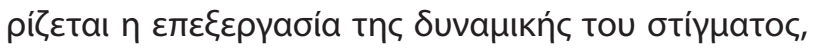

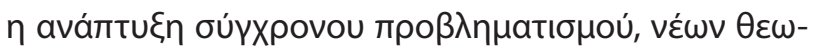

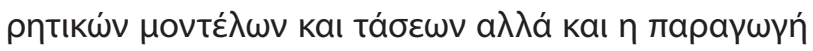

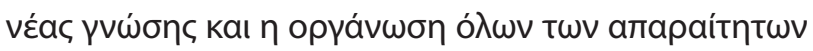

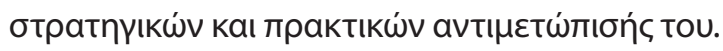

\title{
The stigma of mental illness: \\ A historical overview and conceptual approaches
}

\author{
M. Economou, ${ }^{1,2}$ A. Bechraki, ${ }^{2}$ M. Charitsi ${ }^{2}$ \\ ${ }^{1}$ First Department of Psychiatry, National and Kapodistrian University of Athens, Eginition Hospital, Athens, \\ 2University Mental Health, Neuroscience and Precision Medicine Research Institute "Costas Stefanis" (UMHRI), Athens, Greece
}

Psychiatriki 2020, 31:36-46

The present article explores the concept of stigma from a historical and theoretical perspective. At first, the conceptual origin of the term "stigma" is presented as well as its subsequent course and incorporation in the scientific field. The term stigma originates from ancient Greek language and in

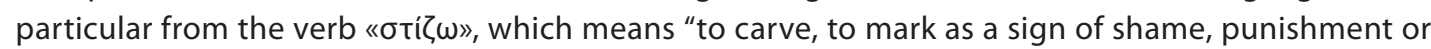
disgrace". In contemporary thinking about stigma, the work of Erving Goffman is seminal. According to him, stigmatization is elicited by the presence of a socially undesirable characteristic, which signals otherness. When this characteristic becomes conspicuous during a social interaction, it may act in a disqualifying manner for the identity of the person who bears it. One of the first theories on social stigma which attracted increased scientific attention is labeling theory by Thomas Scheff. Later 
on, the conceptual model of Corrigan and Watson underscored the main constituents of stigma, namely stereotypes, prejudices and discrimination; whereas the theoretical framework of Link and Phelan stressed labeling, stereotyping, separation, status loss and discrimination as interconnected components in a power situation. During the last half of the previous century, the concept of stigma came to the fore and gained growing research attention, especially due to shedding light on the ways whereby people with mental disorders were treated socially. Most of the literature has focused on recording the general population's level of knowledge and lay beliefs about mental illness as well as on exploring social attitudes and desired social distance from people with mental disorders. Converging evidence indicates that stereotypical beliefs and discriminatory attitudes against people with mental illness prevail worldwide; while illness severity, poor therapeutic outcome, disturbances in patients' emotional expression during a social interaction, incidents of violent or dangerous behaviours and labeling have all been shown to influence public stigma. Regarding lay respondents' correlates of public stigma; male gender, older age, lower socio-economic status, lower educational attainment and residence in semi-urban or rural areas have been linked to unfavourable attitudes towards people with mental disorders; while of outmost importance is personal experience/ familiarity with mental illness.

Key words: Stigma, mental illness, historical overview, conceptual approaches, stereotypes, prejudices, discrimination.

\section{BıßAtoypacpía}

1. Sartorius $\mathrm{N}$. One of the last obstacles to better mental health care: the stigma of mental illness. In: Guimón J, Fischer W, Sartorius N (eds) The image of madness. Karger, Basel, 1999

2. Burton R. The anatomy of melancholy. Faulkner TC, Kiessling NK, Blair RL (eds) Clarendon Press, Oxford, 1989

3. Lopez-lbor JJ. The WPA and the fight against stigma because of mental diseases. World Psychiatry 2002, 1:16-20, PMID: 16946818

4. Sartorius N, Schulze H. Reducing the stigma of mental illness: A report from a global programme of the World Psychiatric Association. Cambridge University Press, New York, 2005

5. Goffman E. Stigma: Notes on the Management of Spoiled Identity. Penguin, Harmondsworth, 1970

6. Scheff TJ. Being mentally ill: A sociological theory. Aldine, Chicago, 1966

7. Goffman E. Stigma. Penguin, London, 1963

8. Jones EE, Farina A, Hastorf AH, Markus H, Miller DT, Scott RA. Social Stigma: The Psychology of Marked Relationships. Freeman, New York, 1984

9. Corrigan PW, Watson AC. Understanding the impact of stigma on people with mental illness. World Psychiatry 2002, 1:16-20, PMID: 16946807

10. Link BG, Phelan JC. Conceptualizing stigma. Annu Rev Sociol 2001, 27:363-385, doi: 10.1146/annurev.soc.27.1.363

11. Weiner B. Judgments of responsibility: A foundation for a theory of social conduct. Guilford Press, New York, 1995

12. Corrigan PW. Mental health stigma as social attribution: Implications for research methods and attitude change. Clin Psychol Sci Pract 2000, 7:48-67, doi:10.1093/clipsy.7.1.48

13. Corrigan PW, River LP, Lundin RK, Uphoff Wasowski K, Campion $\mathrm{J}$, Mathisen $\mathrm{H}$ et al. Stigmatizing attributions about mental ill- ness. J Community Psychol 2000, 28:91-102, doi: 10.1002/ (SICI)1520-6629(200001)28:1<91::AID-JCOP9>3.0.CO;2-M

14. Corrigan PW, Watson AC, Heyrman M, Warpinski A, Gracia G, Slopen $\mathrm{N}$ et al. Structural stigma in state legislation. Psychiatr Serv 2005, 56:557-563, doi:10.1176/appi.ps.56.5.557

15. Phelan JC, Bromet EJ, Link BG. Psychiatric illness and family stigma. Schizophr Bull 1998, 24:115-126, doi: 10.1093/oxfordjournals.schbul.a033304

16. Persaud R. Psychiatrists suffer from stigma too. B J Psych Bull 2000, 24:284-285, doi: 10.1192/pb.24.8.284

17. Link BG, Phelan JC. Stigma and its public health implications. Lancet 2006, 367:528-529, doi: 10.1016/S0140-6736 (06)68184-1

18. Star S. The publics' ideas about mental illness. Paper presented at the Annual Meeting of the National Association for Mental Health, 5 November 1955. Indianapolis

19. Cumming E, Cumming G. Closed ranks: an experiment in mental health education. Harvard University Press, Cambridge, 1957

20. Whatley C. Social attitudes towards discharged patients. Soc Problems 1959, 6:313-320, doi: 10.2307/799364

21. Bogardus EM. Measuring social distance. J Appl Sociol 1925, 9: 299-308

22. Owen CA, Eisner HC, McFaul TR. A half century of social distance research: National replication of the Bogardus' studies. Sociol Soc Res 1981, 66:80-98

23. Angermeyer MC, Matschinger $\mathrm{H}$. Social distance towards the mentally ill: results of representative survey in the Federal republic of Germany. Psychol Med 1997, 27:131-141, doi: 10.1017/s0033291796004205

24. Angermeyer MC, Beck M, Matschinger H. Determinants of the public's preference for social distance from people with schizo- 
phrenia. Can J Psychiatry 2003, 48: 663-668, doi: 10.1177/ 070674370304801004

25. Crocetti G, Spiro JR, Siassi I. Are the ranks closed? Attitudinal social distance and mental illness. Am J Psychiatry 1971, 127: 1121-1127

26. Eker D. Attitudes toward mental illness: recognition, desired social distance, expected burden and negative influence on mental health among Turkish freshmen. Soc Psychiatry Psychiatr Epidemiol 1989, 24:146-150, doi: 10.1007/bf01788024

27. Brockington $\mathrm{I}$, Hall $\mathrm{P}$, Levings $\mathrm{J}$ et al. The community's tolerance of the mentally ill. Br J Psychiatry 1993, 162:93-99, doi: 10.1192/bjp.162.1.93

28. Madianos M, Economou M, Hatjiandreou $M$ et al. Changes in public attitudes towards mental illness in the Athens area (1979/1980-1994). Acta Psychiatr Scand 1999, 99:73-78, doi: 10.1111/j.1600-0447.1999.tb05387.x

29. Angermeyer MC, Dietrich S. Public beliefs about and attitudes towards people with mental illness: a review of population studies. Acta Psychiatr Scand 2006, 113:163-179, doi: 10.1111/j.1600-0447.2005.00699.x

30. Martin JK, Pescosolido BA, Tuch SA. Of fear and loathing: The role of "disturbing behavior", labels and causal attributions in shaping public attitudes toward people with mental illness. $J$ Health Soc Behav 2000, 41: 208-223, doi: 10.2307/2676306

31. Angermeyer MC, Matschinger H, Corrigan P. Familiarity with mental illness and social distance from people with schizophrenia and major depression: Testing a model using data from a representative population survey. Schizophr Res 2004, 69: 175-182, doi: 10.1016/s0920-9964(03)00186-5

32. Magliano L, Fiorillo A, de Rosa C et al. Beliefs about schizophrenia in Italy: a comparative nationwide survey of the general public, mental health professionals, and patients' relatives. Can J Psychiatry 2004, 49:322-330, doi: 10.1177/070674370404900508

33. Lauber $C$, Nordt $C$, Falcato $L$ et al. Factors influencing social distance toward people with mental illness. Commun Ment Health J 2004, 40:265-274, doi: 10.1023/B:COMH.00000269 99.87728.2d

34. Stuart H, Arboleda-Florez J. Community attitudes towards people with schizophrenia. Can J Psychiatry 2001, 46:245-252, doi: 10.1177/070674370104600304

35. Gaebel W, Baumann A, Witte AM et al. Public attitudes towards people with mental illness in two German cities. Results of a public survey under special considerations of schizophrenia. Eur Arch Psychiatry Clin Neurosci 2002, 252:278-287, doi: 10.1007/s00406-002-0393-2

36. Thompson A, Stuart H, Bland RC et al. Attitudes about schizophrenia from the pilot site of the WPA worldwide campaign against the stigma of schizophrenia. Soc Psychiatry Psychiatr Epidemiol 2002, 37:475-482, doi: 10.1007/s00127-002-0583-2

37. Economou M, Richardson C, Gramandani C, Stalikas A, Stefanis C. Knowledge about schizophrenia and attitudes towards people with schizophrenia in Greece. Int J Soc Psychiatry 2009, 55:361-371, doi: 10.1177/0020764008093957

38. Mouzas OD, Angelopoulos NV, Liakos A. Public opinions about mental illness in a Greek area: The influence of sociodemographic factors. Psychiatriki 2008, 19:337-349, PMID: 22218082
39. Arvanity A. Public attitudes towards mentally ill. Democritus University of Thrace 2008 (Cited 23 November 2019). Available from http://thesis.ekt.gr/thesisBook Reader/id/21014\#page/1/ mode/2up

40. Melissa C, Mavraki C, Gourni M, Tsalkanis A, Pilatis N, Argyriou $G$ et al. People's attitudes towards patients with mental illness in Greece. ICUs Nurs Web J 2006, 27:1-12

41. Tzouvara V, Papadopoulos C. Public stigma towards mental illness in the Greek culture. J Psychiatr Ment Health Nurs 2014, 21:931-938, doi: 10.1111/jpm.12146

42. Anagnostopoulos F, Hantzi A. Familiarity with and social distance from people with mental illness: Testing the mediating effects of prejudiced attitudes. J Community Appl Soc Psychol 2011, 21:451-460, doi: 10.1002/casp.1082

43. Saridi M, Kordosi A, Toska A, Peppou LE, Economou M, Souliotis K. Attitudes of health professionals towards the stigma surrounding depression in times of economic crisis. Int J Soc Psychiatry 2017, 63:115-125, doi: 10.1177/0020764016685348

44. Link BG, Cullen FT, Frank J, Wozniak JF. The social rejection of former mental patients: Understanding why labels matter. Am J Soc 1987, 92:1461-1500, doi: 10.1086/228672

45. Read J, Law A. The relationship of causal beliefs and contact with users of mental health services to attitudes to the 'mentally ill'. Int J Soc Psychiatry 1999, 45:216-229, doi: 10.1177/002076409904500309

46. Sartorius N, Schulze H. Reducing the stigma of mental illness: A report from a global programme of the World Psychiatric Association. Cambridge University Press, New York, 2005

47. Crisp AH, Gelder M, Rix S, Meltzer HI, Rowlands OJ. Stigmatization of people with mental illnesses. Br J Psychiatry 2000, 177:4-7, doi: 10.1192/bjp.177.1.4

48. Sugiura T, Sakamoto S, Tanaka E et al. Labeling effect of Seishin-bunretsu-byou, the Japanese translation for schizophrenia: an argument for relabeling. Int J Soc Psychiatry 2001, 47:43-51, doi: 10.1177/002076400104700204

49. Link BG, Phelan JC, Bresnahan M, Stueve A, Pescosolido BA. Public conceptions of mental illness: Labels causes, dangerousness and social distance. Am J Publ Health 1999, 89:13281333, doi: 10.2105/ajph.89.9.1328

50. Penn D, Guynan K, Daily T, Spaulding WD, Garbin CP, Sullivan $M$. Dispelling the stigma of schizophrenia: what sort of information is best? Schizophr Bull 1994, 20:567-578, doi: 10.1093/ schbul/20.3.567

51. Corrigan PW, Watson, AC. The stigma of psychiatric disorders and the gender, ethnicity, and education of the perceiver. Community Ment Health J 2007, 43:439-458, doi: 10.1007/ s10597-007-9084-9

52. Townley G, Brusilovskiy E, Salzer M. Urban and non-urban differences in community living and participation among individuals with serious mental illnesses. Soc Sci Med 2017, 177: 223-230, doi: 10.1016/j.socscimed.2017.01.058

53. Buizza C, Ghilardi A, Ferrari C. Beliefs and prejudices versus knowledge and awareness: how to cope with stigma against mental illness. A college staff e-survey. Community Ment Health J 2017, 53: 589-597, doi: 10.1007/s10597-017-0116-9 
54. Link BG, Cullen FT. Contact with the mentally ill and perceptions of how dangerous they are. J Health Soc Behav 1986, 27:289-302, PMID: 3559124

55. Markovitz FA, Engelman DJ. The "Own" and the "Wise": Does stigma status buffer or exacerbate social rejection of college students with a mental illness? Deviant Behav 2017, 38:744-755, doi: 10.1080/01639625.2016.1197673

56. Zen G, Lepri B, Ricci E, Lanz O. Space speaks: towards socially and personality aware visual surveillance. Paper presented at 1st ACM International Workshop on Multimodal Pervasive Video Analysis (MPVA 2010), 29 October 2010. Firenze, MPVA'10 Proceedings of the 2010 ACM Workshop on Multimodal Pervasive Video Analysis, pp. 37-42, doi: 10.1145/1878039.1878048

57. Schulze B, Angermeyer MC. Subjective experiences of stigma. A focus group study of schizophrenic patients, their relatives and mental health professionals. Soc Sci Med 2003, 56:299312, doi: 10.1016/s0277-9536(02)00028-x

58. Gaebel W, Zäske H, Bauman AE. The relationship between mental illness severity and stigma. Acta Psychiatr Scand Suppl 2006, 113:41-45, doi: 10.1111/j.1600-0447.2005.00716.x

59. Bauman AE, Craige E, Zäske $H$ et al. Interpersonal factors contributing to the desire for social distance. Paper presented at the XIIIth World Congress of Psychiatry, 10-15 September 2005. Cairo

60. Grausgruber A, Meise U, Katsching H, Schöny W, Fleischhacker WW. Patterns of social distance towards people suffering from schizophrenia in Austria: a comparison between the general public, relatives and mental health staff. Acta Psychiatr Scan 2007, 115:310-319, doi: 10.1111/j.1600-0447.2006.00882.x

61. Crisp AH, Gelder M, Rix S, Meltzer HI, Rowlands OJ. Stigmatization of people with mental illnesses. Br J Psychiatry 2000, 177:4-7, doi: 10.1192/bjp.177.1.4

62. Marwaha S, Johnson S. Schizophrenia and employment: A review. Soc Psychiatry Psychiatr Epidemiol 2004, 39:337-349, doi: 10.1007/s00127-004-0762-4

63. Barney LJ, Griffiths KM, Jorm A, Christensen H. Stigma about depression and its impact on help-seeking intentions. Aust N Z J Psychiatry 2006, 40: 51-54, doi: 10.1080/j.1440-1614. 2006.01741.x

64. Clement S, Schauman O, Graham T, Maggioni F, Evans-Lacko $\mathrm{S}$, Bezborodovs $\mathrm{N}$ et al. What is the impact of mental health stigma on help-seeking? A systematic review of quantitative and qualitative studies. Psychol Med 2015, 45:11-27, doi: 10.1017/S0033291714000129

65. Livingston JD, Boyd JE. Correlates and consequences of internalized stigma for people living with mental illness: a systematic review and meta-analysis. Soc Sci Med 2010, 71: 2150-2161, doi: 10.1016/j.socscimed.2010.09.030

66. Boyd JE, Adler EP, Otiligam PG, Peters T. Internalized stigma of mental illness (ISMI) Scale: a multinational review. Compr Psychiatry 2014, 55: 221-231, doi: 10.1016/j.comppsych. 2013.06.005

67. Yanos PT, Lysaker PH, Roe D. Internalized stigma as a barrier to improvement in vocational functioning among people with schizophrenia-spectrum disorders. Psychiatry Res 2010 (a), 178: 211-213, doi: 10.1016/j.psychres.2010.01.003

68. Yanos PT, Lysaker PH, Roe D. The impact of illness identity on recovery from severe mental illness. Am J Psychiatr Rehabil 2010(b), 13:73-93, doi: 10.1080/15487761003756860

69. Oexle N, Muller M, Kawohl W, Xu Z, Viering S, Wyss C et al. Self-stigma as a barrier to recovery: a longitudinal study. Eur Arch Psychiatry Clin Neurosci 2018, 268:209-212, doi: 10.1007/ s00406-017-0773-2

70. Oexle N, Rusch N, Viering S, Wysss C, Seifritz E. Self-stigma and suicidality: a longitudinal study. Eur Arch Psychiatry Clin Neurosci 2017, 267: 359-361, doi: 10.1007/s00406-016-0698-1

71. Yanos PT, Roe D, Markus K, Lysaker PH. Pathways between internalized stigma and outcomes related to recovery in schizophrenia spectrum disorders. Psychiatr Serv 2008, 59:1437-1442, doi: 10.1176/appi.ps.59.12.1437

72. Munoz M, Sanz M, Perez-Santos E, Quiroga MA. Proposal of a socio-cognitive-behavioral structural equation model of internalized stigma in people with severe and persistent mental illness. Psychiatr Res 2011, 186: 402-408, doi: 10.1016/j.psychres. 2010.06.019

73. Corrigan PW. How clinical diagnosis might exacerbate the stigma of mental illness. Soc Work 2007, 52:31-39, doi: 10.1093/ $\mathrm{sw} / 52.1 .31$

74. Zartaloudi A, Madianos M. Stigma related to help-seeking from a mental health professional. Health Sci J 2010, 4:77-83

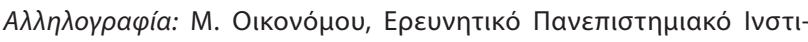

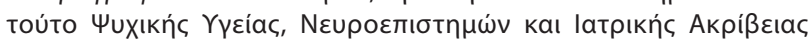

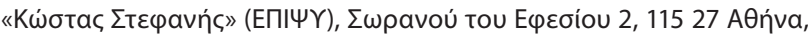
$\operatorname{T\eta } \lambda:$ 210-61 70 071-2, 6170 804-5, Fax: 210-65 64021

e-mail: antistigma@epipsi.eu, marinaeconomou1@gmail.com 


\title{
Avaбкómnon Review
}

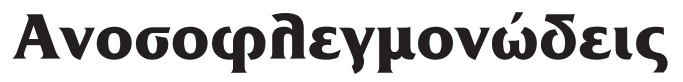

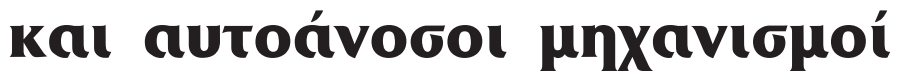

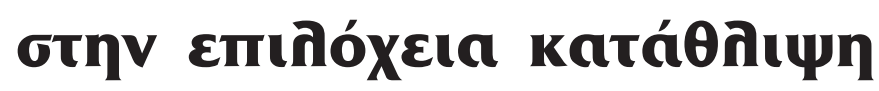

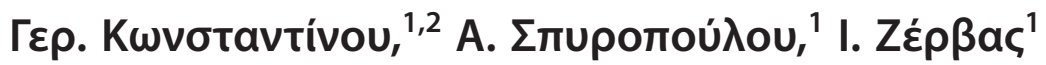

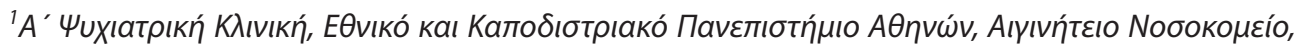

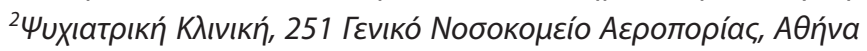

Uuxıатрıкń 2020, 31:47-56

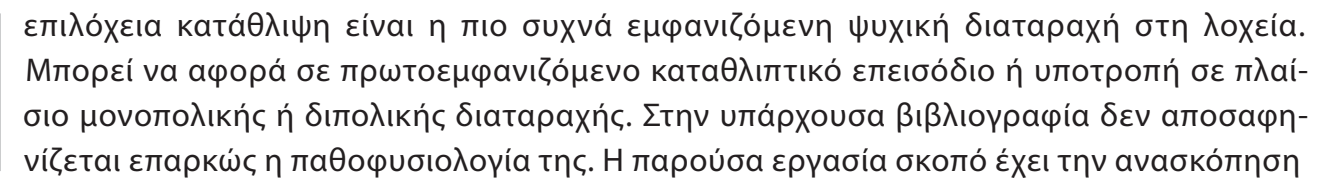

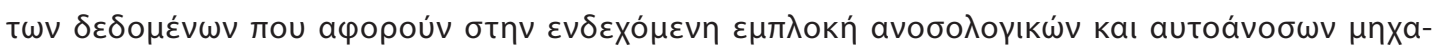

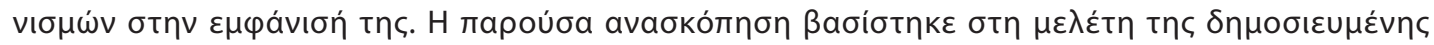

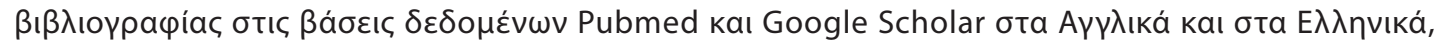

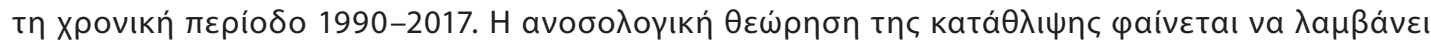

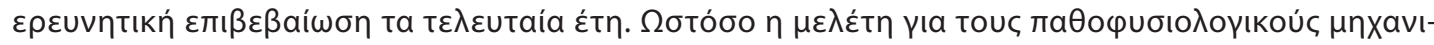

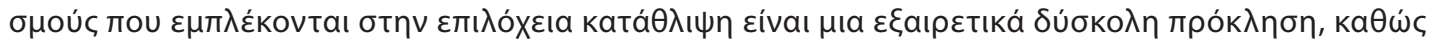

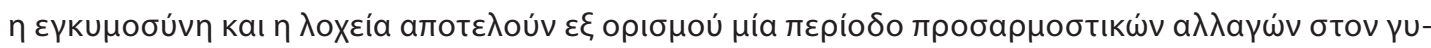

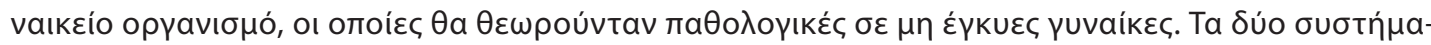

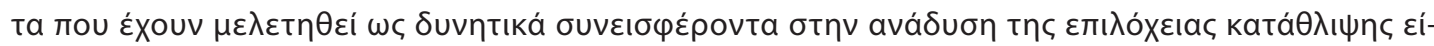

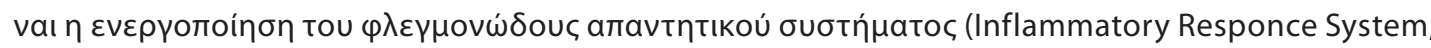

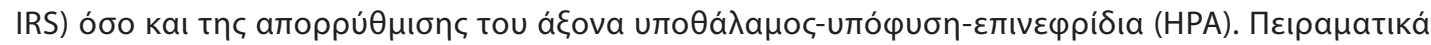

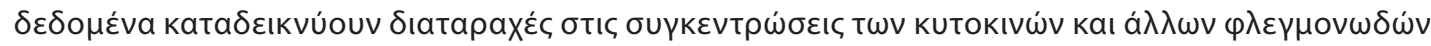

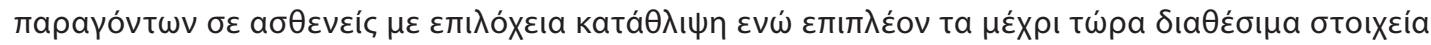

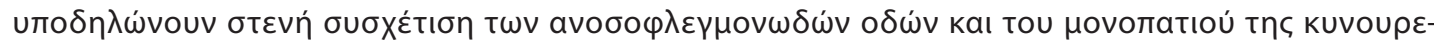

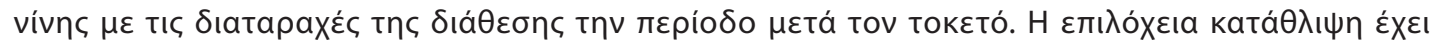

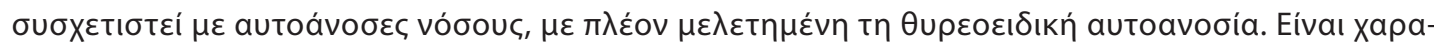

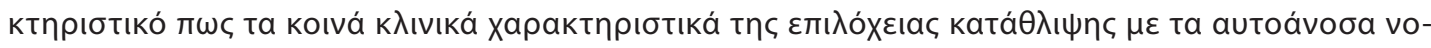




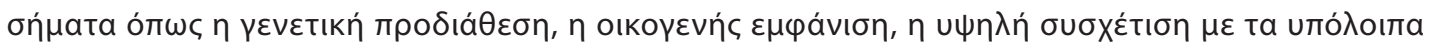

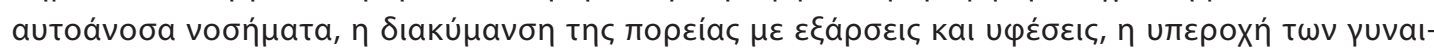

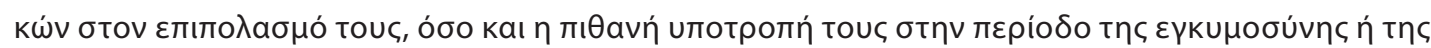

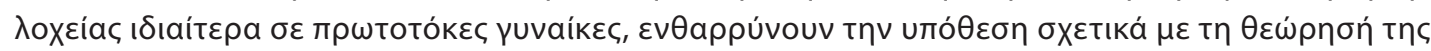

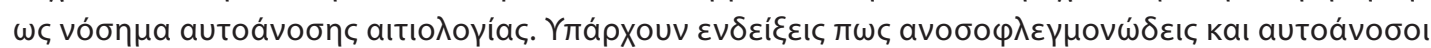

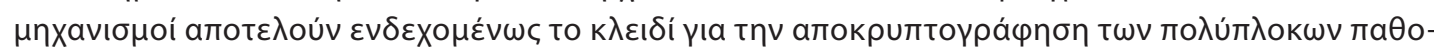

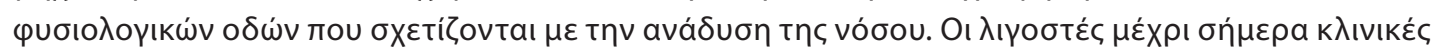

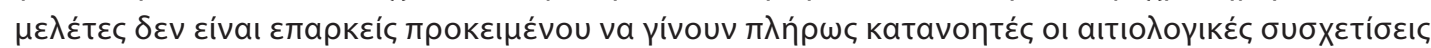

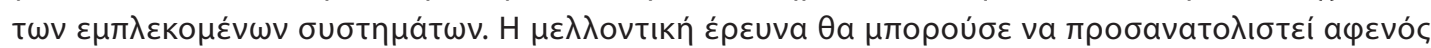

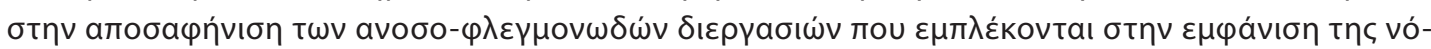

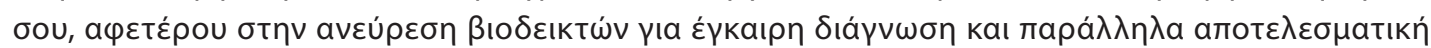

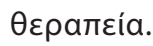

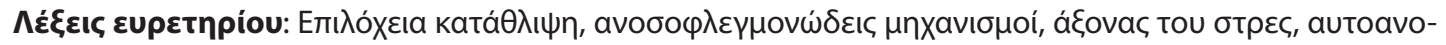

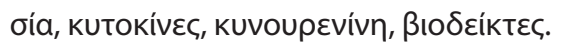

\section{Eıбaywyń}

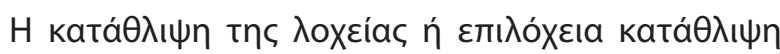

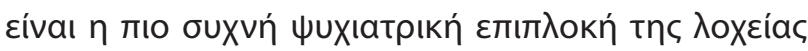

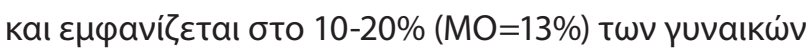

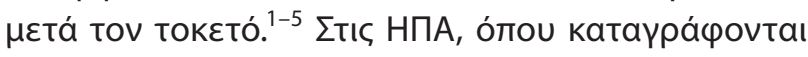

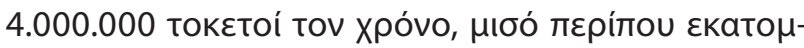

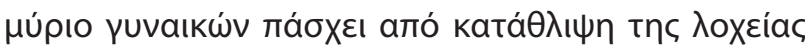

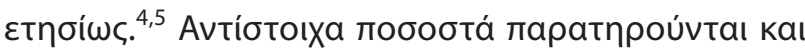

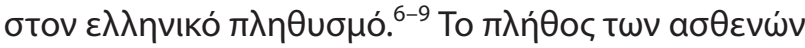

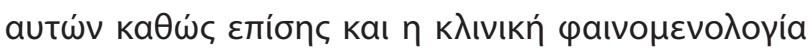

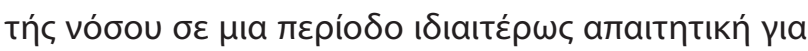

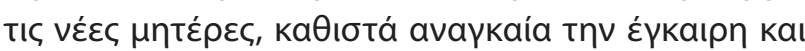

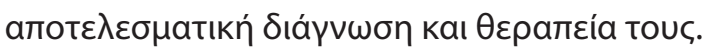

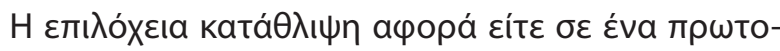

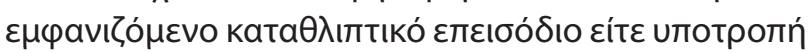

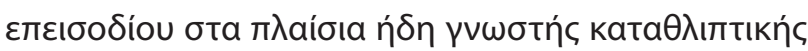

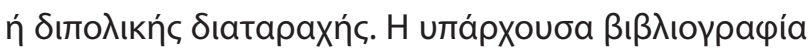

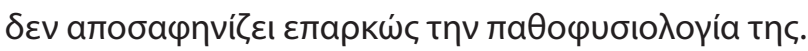

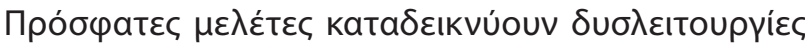

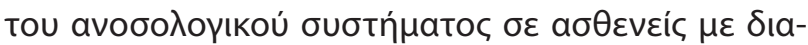

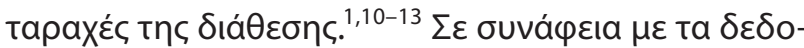

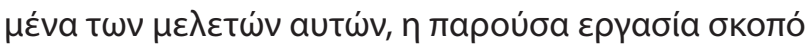

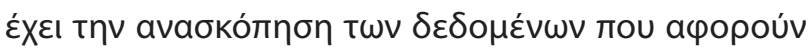

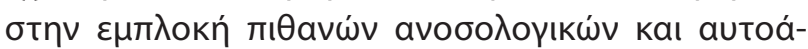

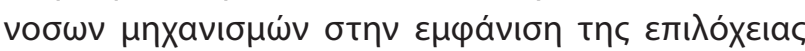

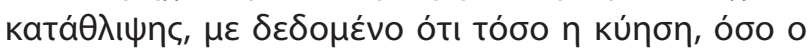

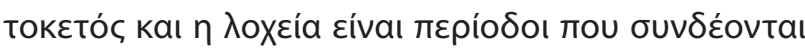

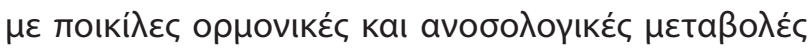

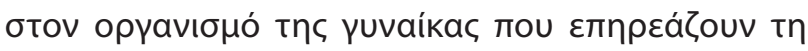

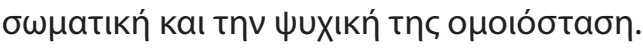

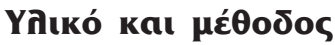

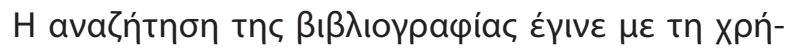

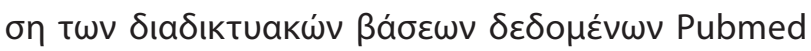

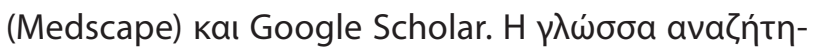

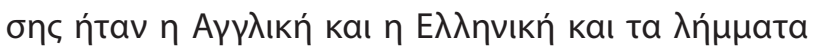

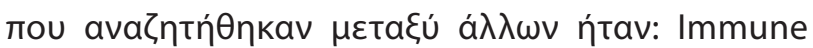
And Postpartum Depression, Autoimmunity And Depression, Psychoneuroimmunology And Postpartum, Immune And Pregnancy, HPA Axis And Depression, HPA axis And Postpartum. H avaל̧́ñnon

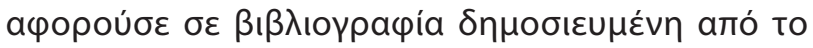

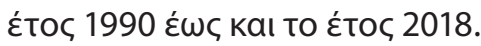

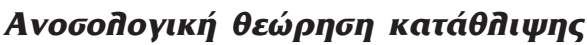

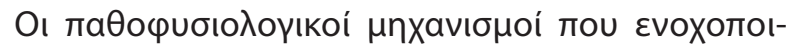

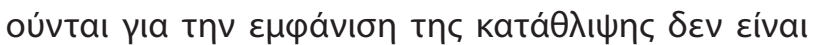

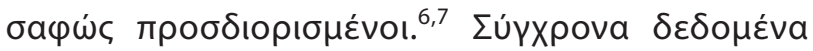

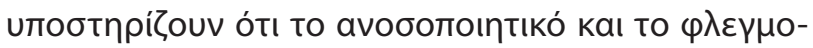

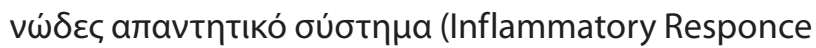

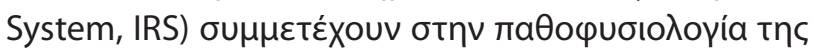

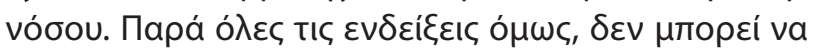

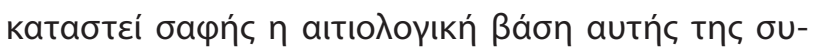

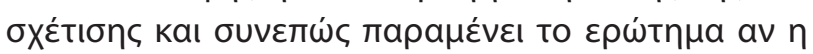

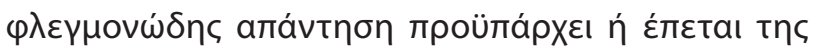
Sıatapaxńc.

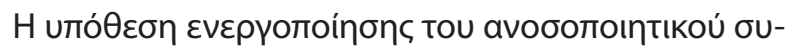

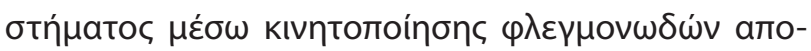

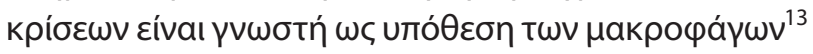

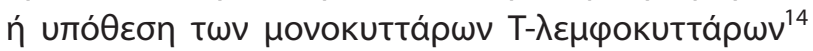

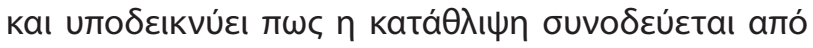




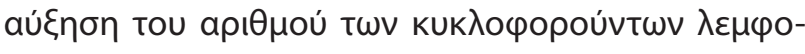

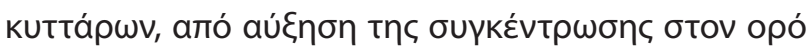

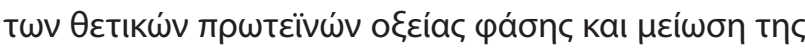

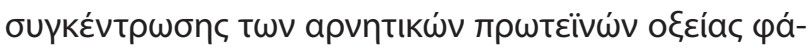

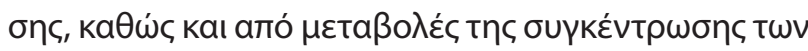

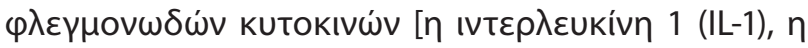

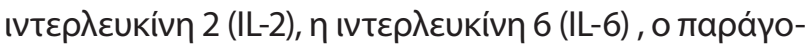

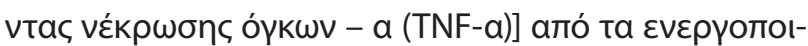

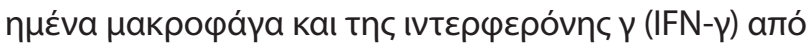

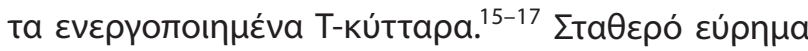

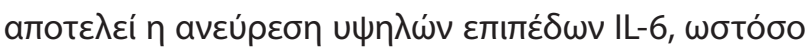

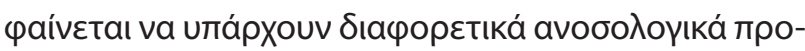

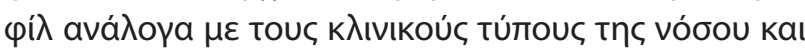

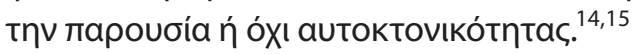

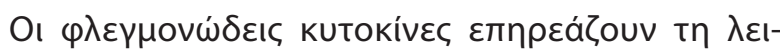

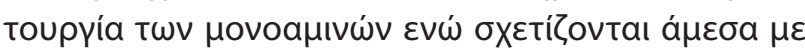

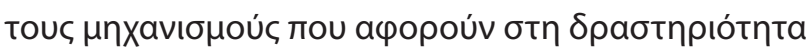

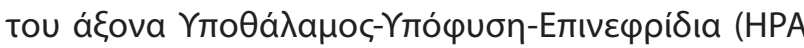

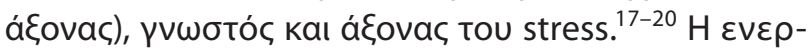

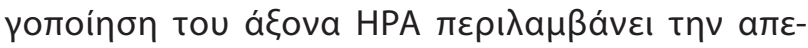

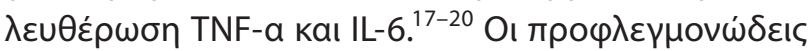

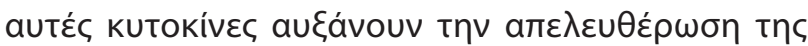

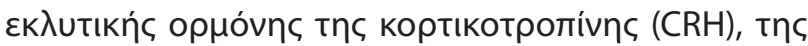
$\varphi \lambda$ oı

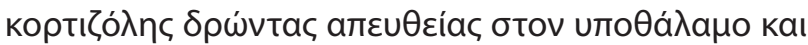

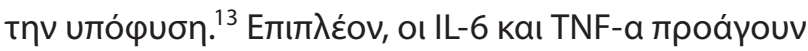

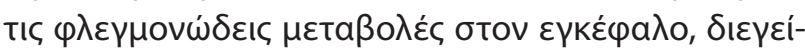

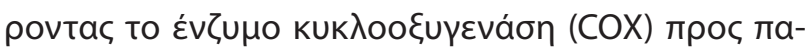

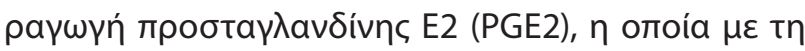

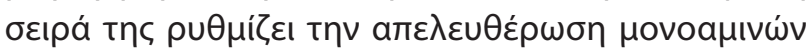

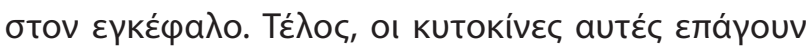

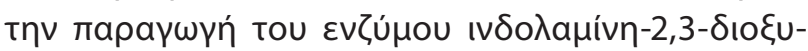

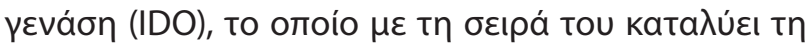

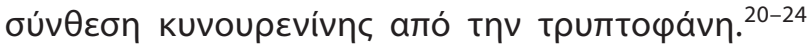

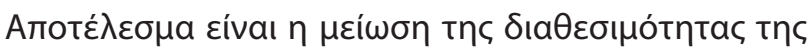

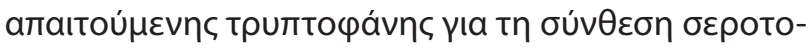

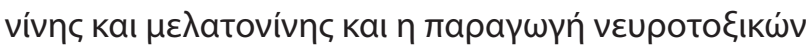

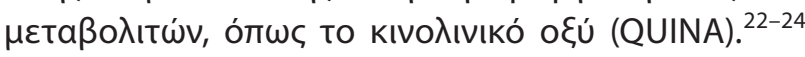

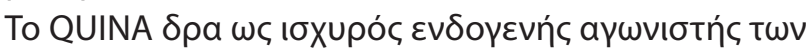

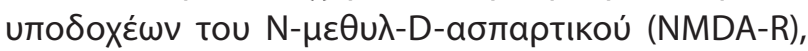

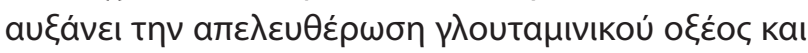

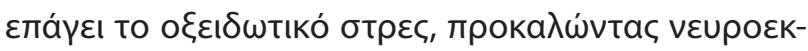

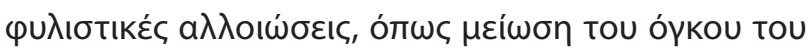
เттока́нтои. ${ }^{14}$

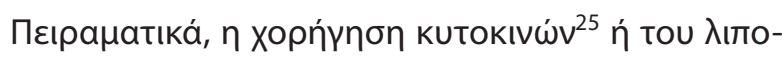

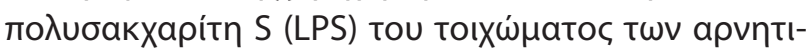

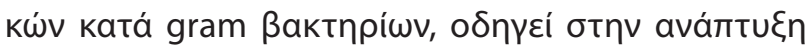

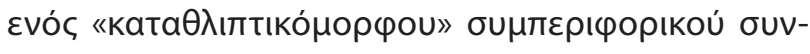

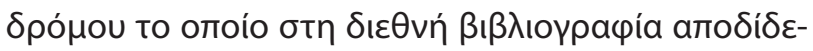

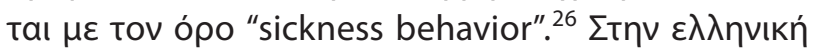

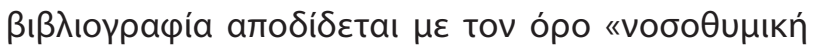

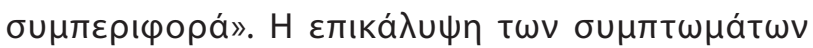

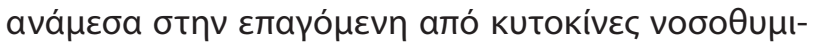

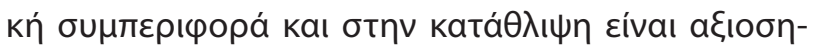

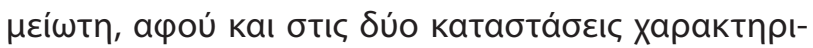

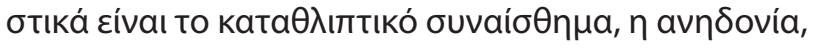

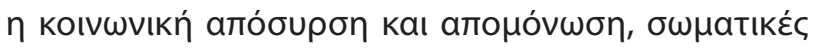

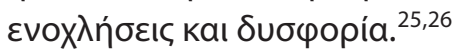

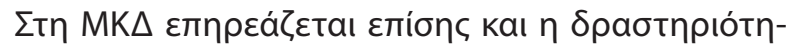

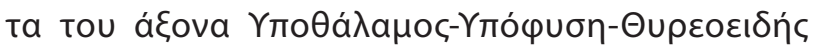

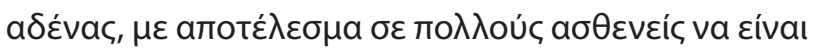

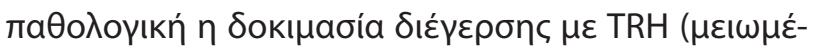

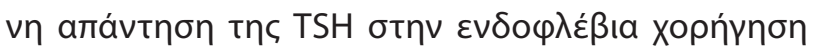

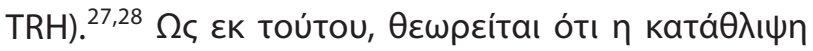

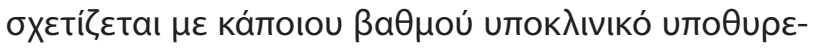
овıઠı

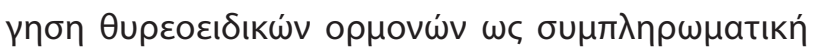

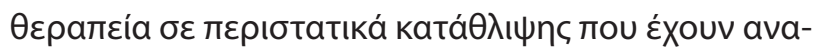

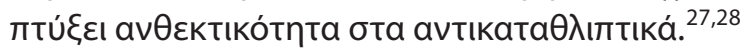

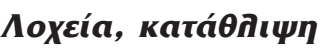

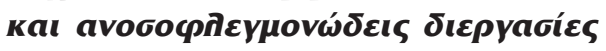

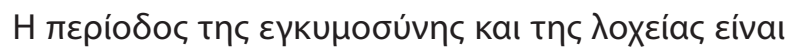

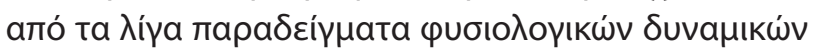

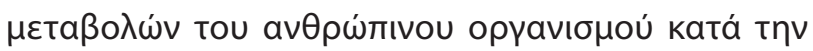

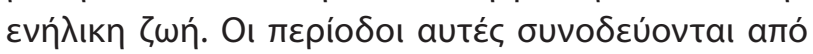

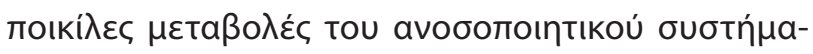

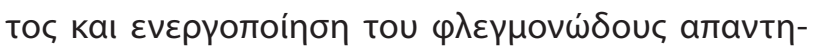

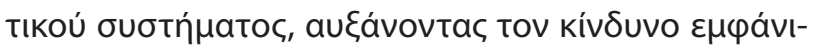

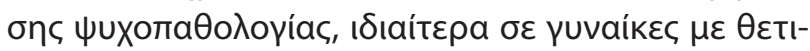

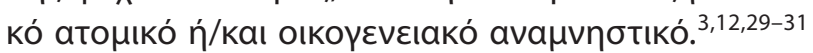

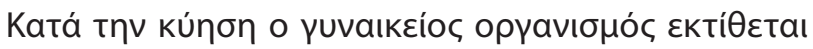

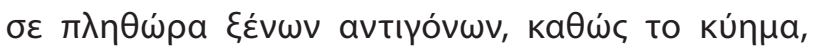

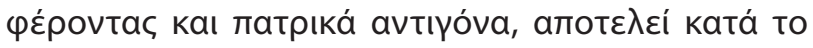

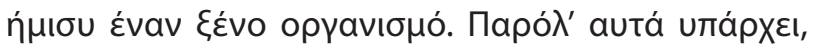

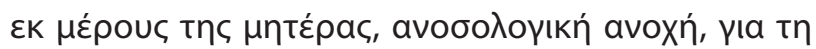

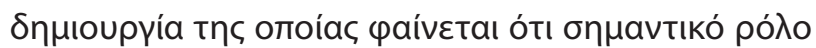

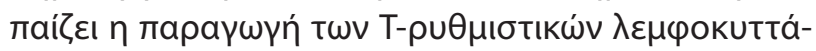

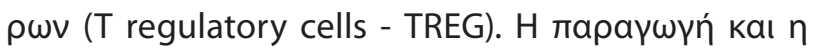

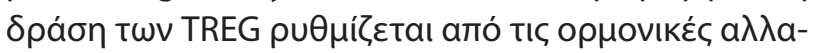

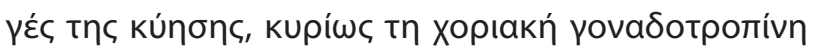

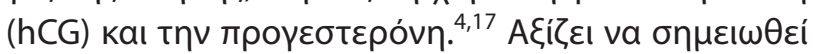

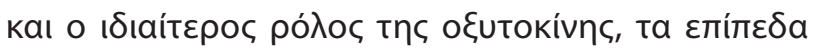

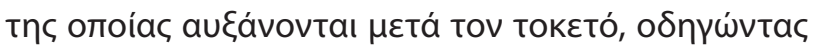

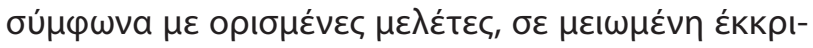




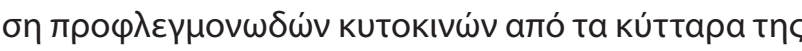

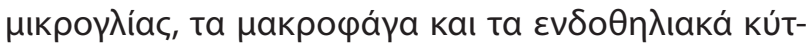
Tapa. $^{32,33}$

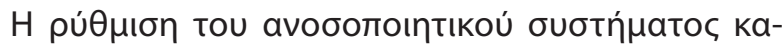

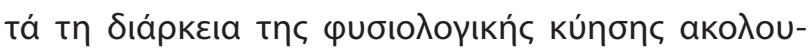

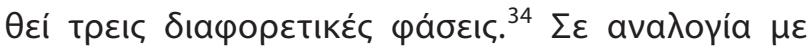

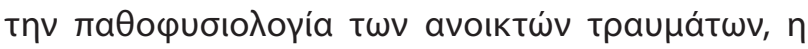

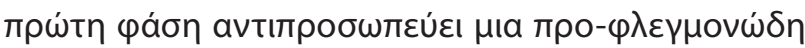

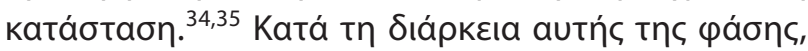

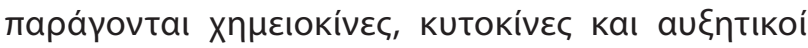

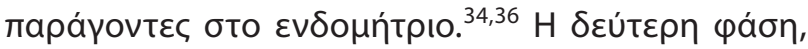

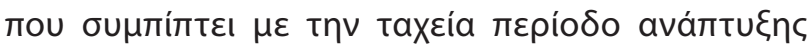

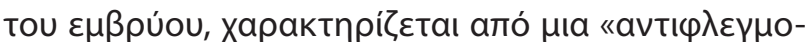

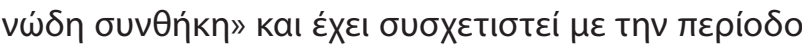

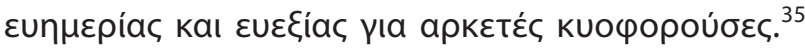

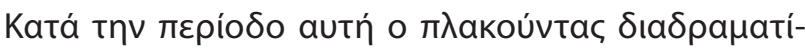

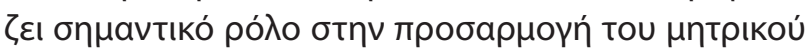

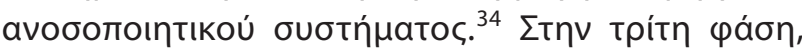

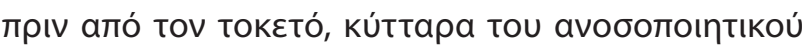

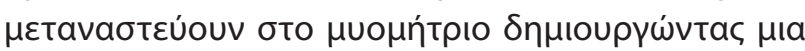

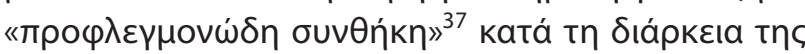

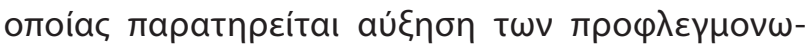

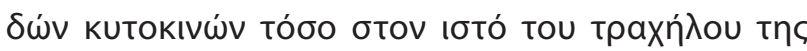

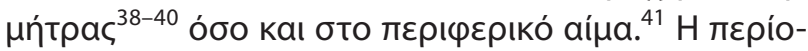

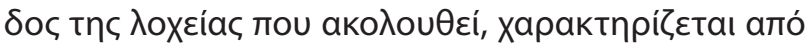

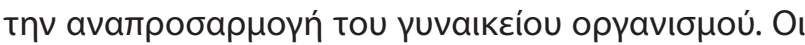

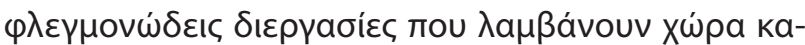

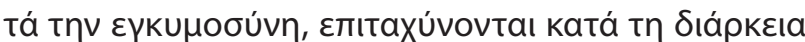

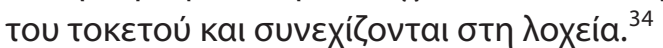

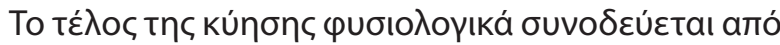

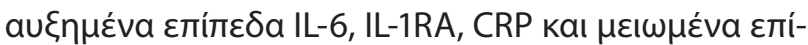

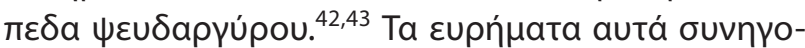

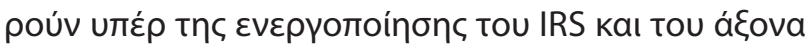

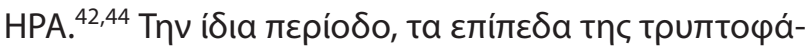

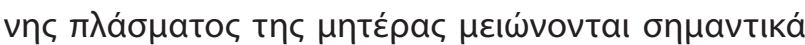

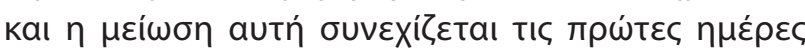

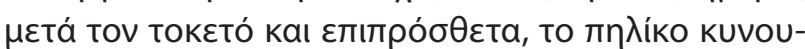

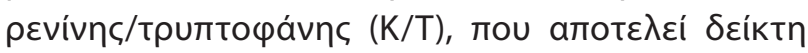

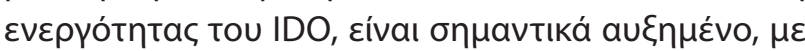

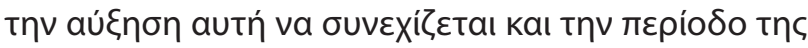
$\lambda_{\text {oxéá. }}{ }^{22}$

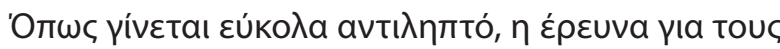

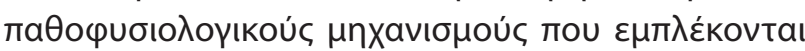

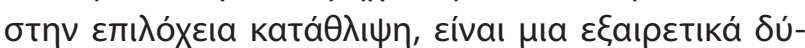

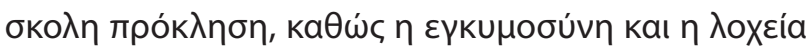

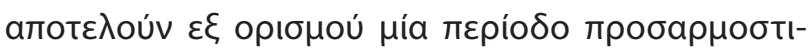

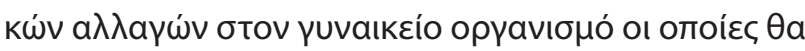

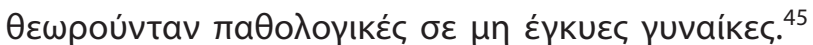

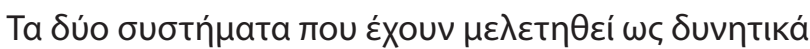

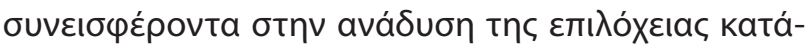

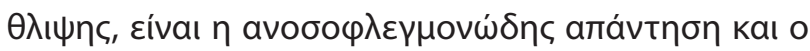

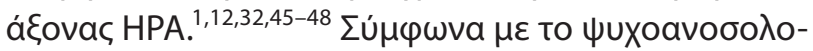

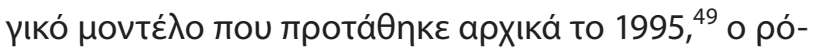

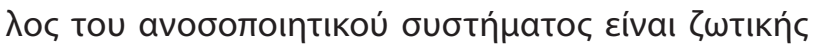

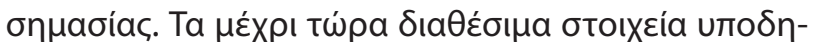

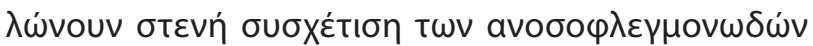

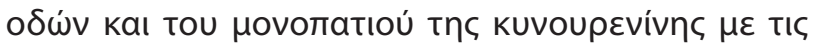

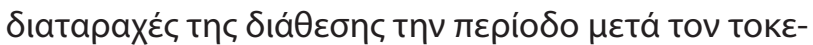

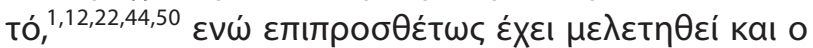

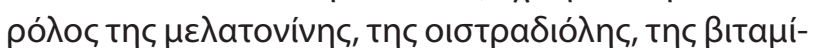

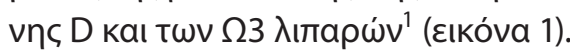

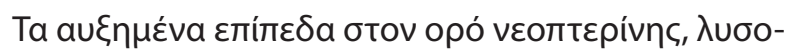

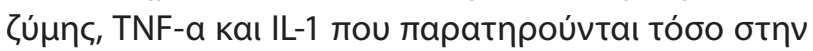

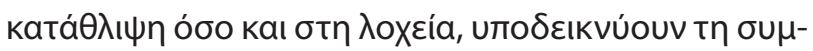

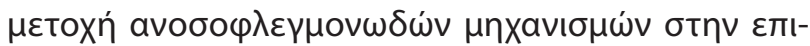

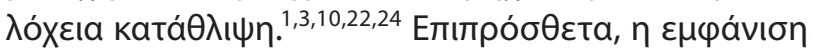

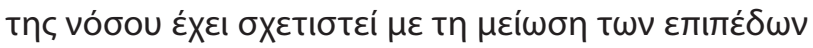

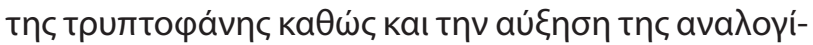

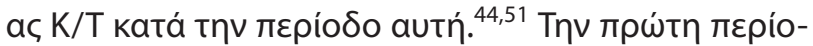

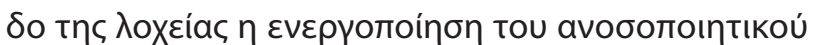

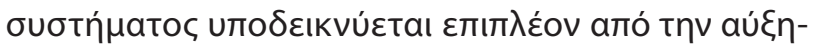

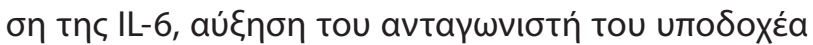

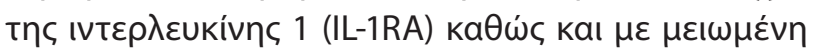

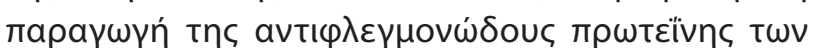

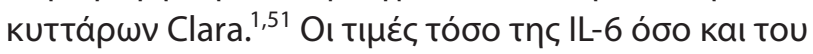

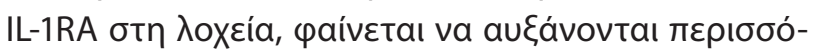

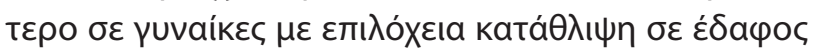

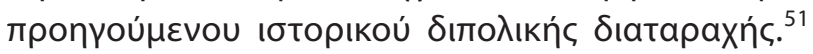

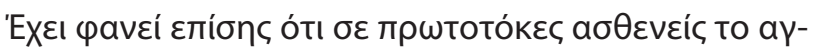

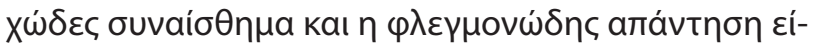

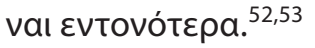

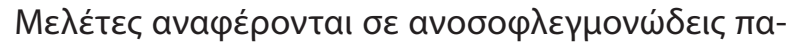

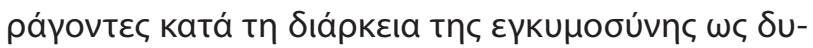

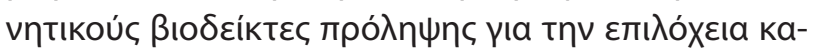

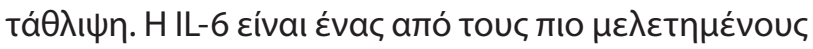

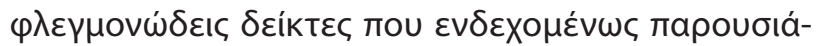

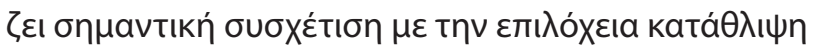

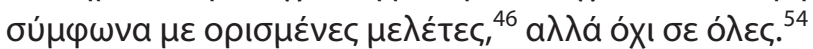

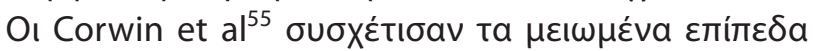

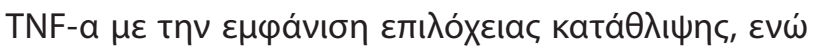

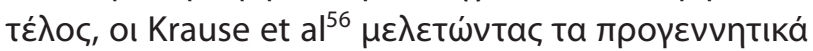

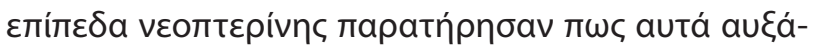

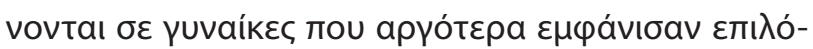
хعıа катá $\theta \lambda ı \psi \eta$. 


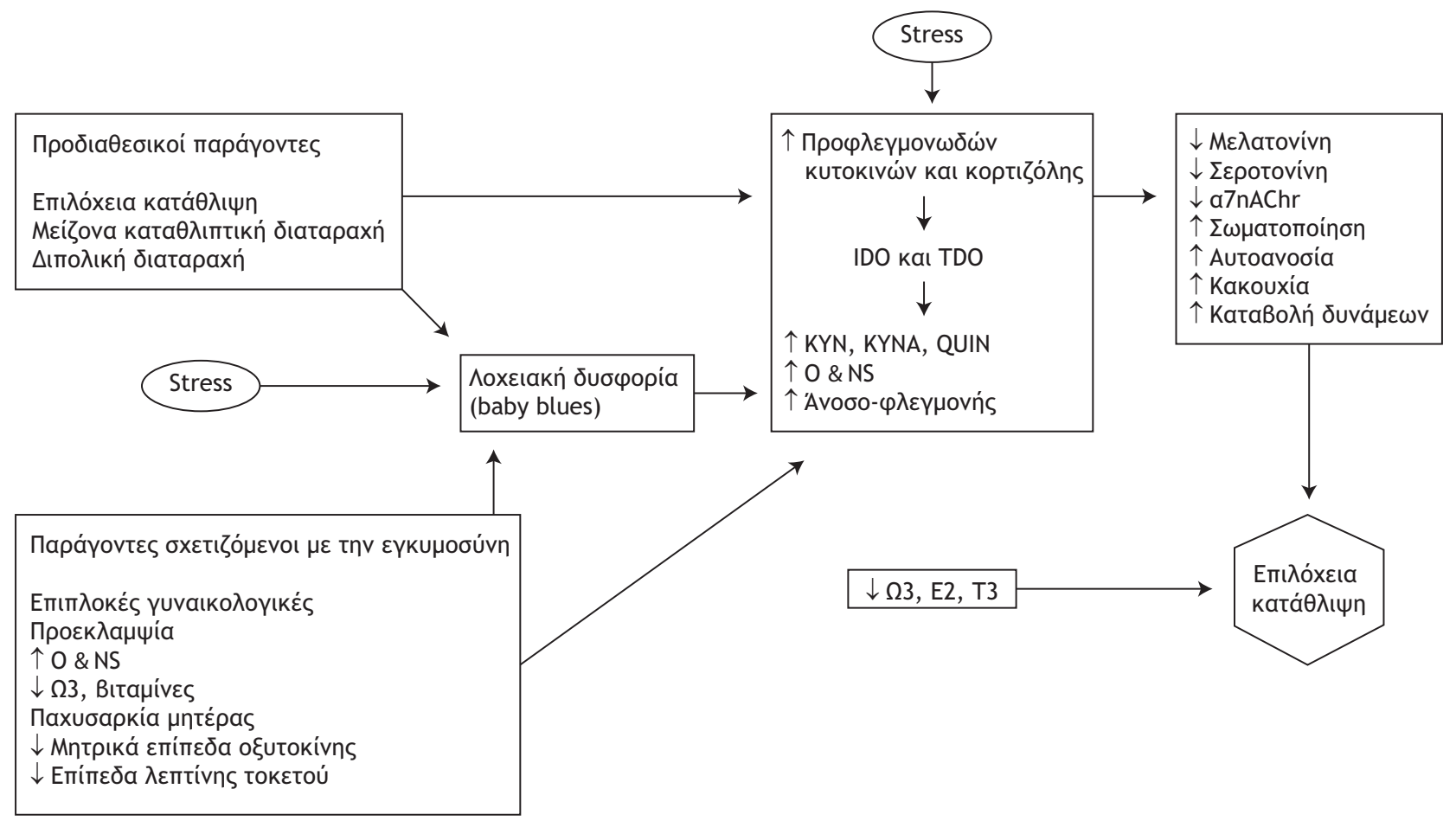

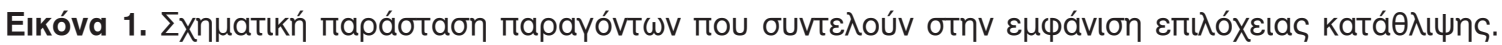

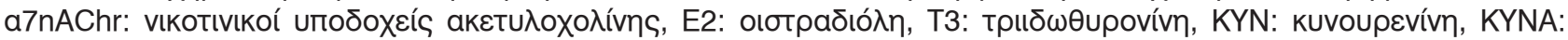

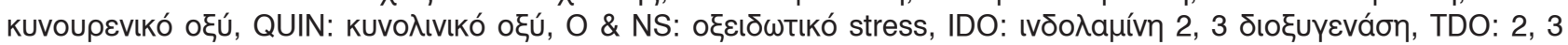

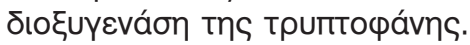

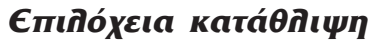

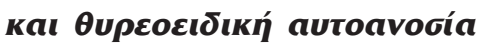

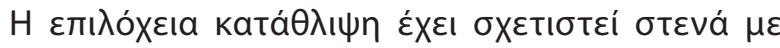

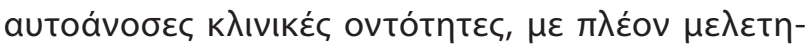

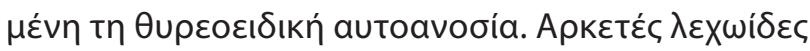

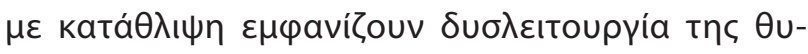

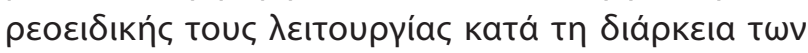

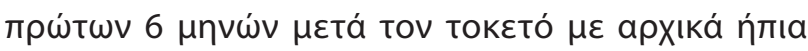

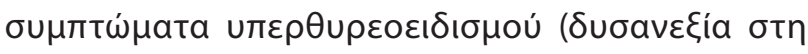

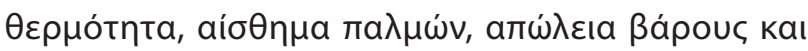

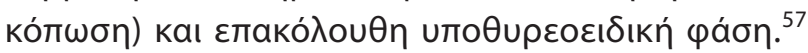

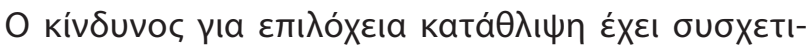

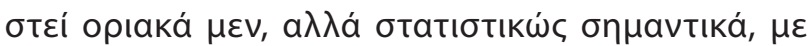

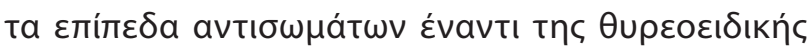

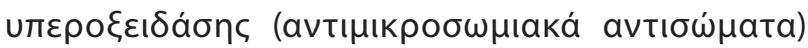

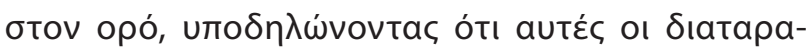

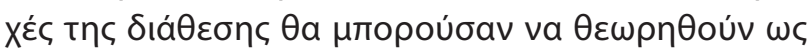

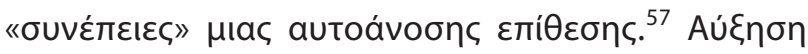

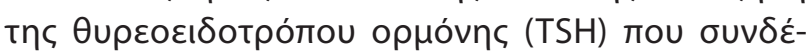

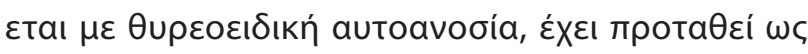

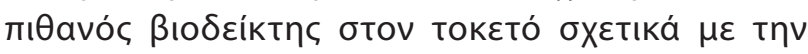

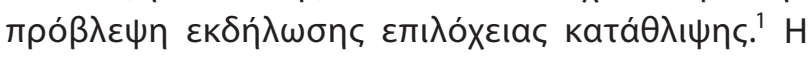

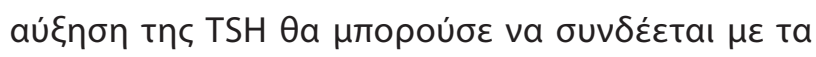

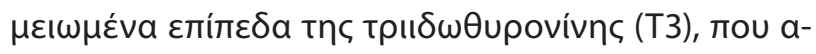

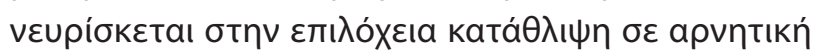

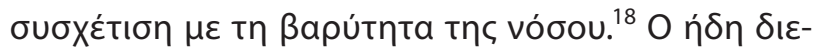

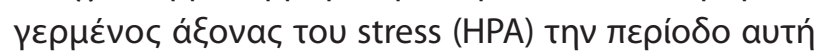

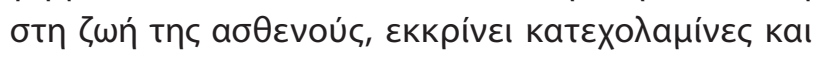

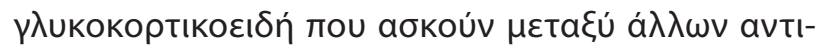

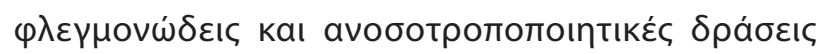

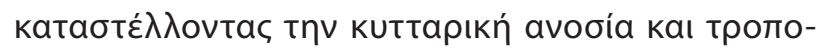

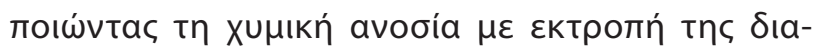

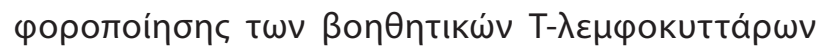

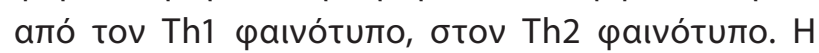

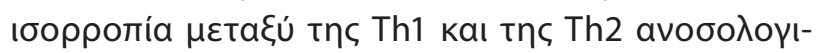

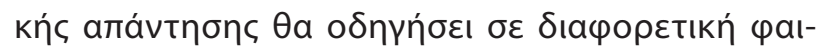

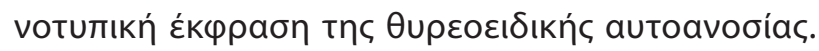

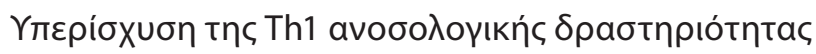

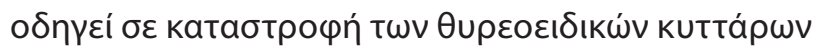

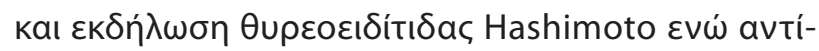

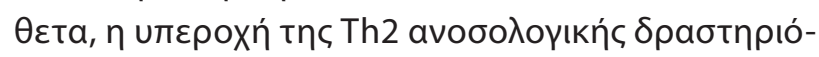

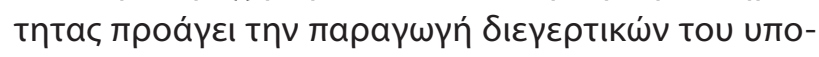

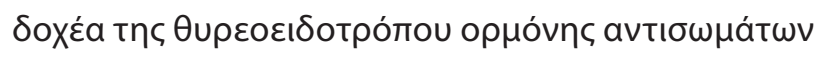

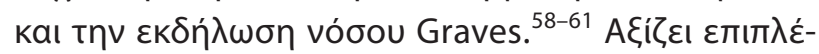




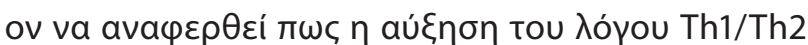

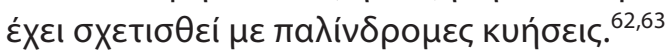

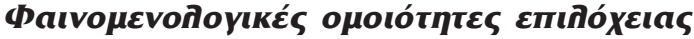

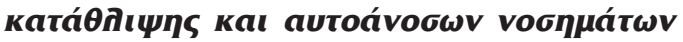

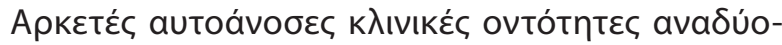

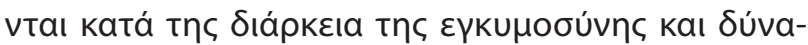

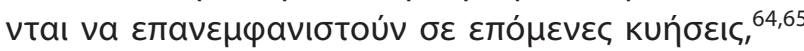

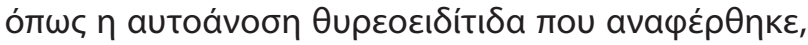

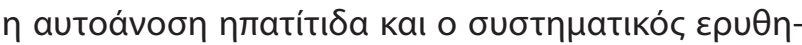

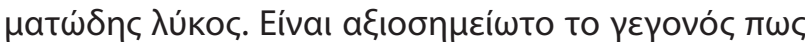

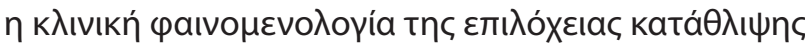

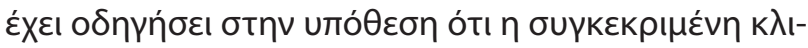

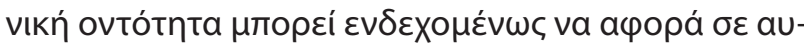

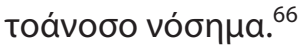

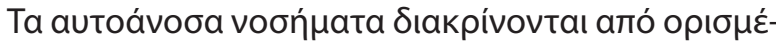

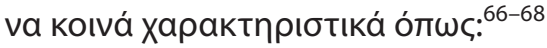

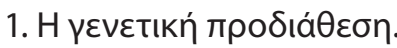

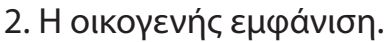

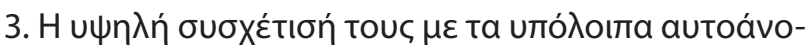
бa voońmata.

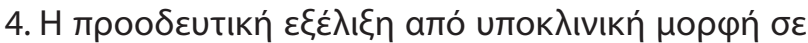

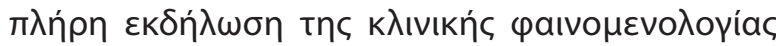
Touc.

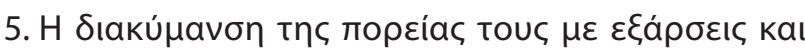

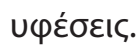

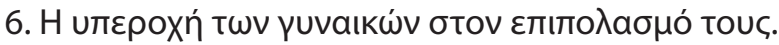

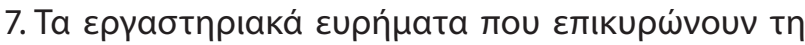

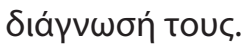

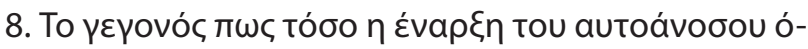

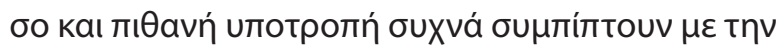

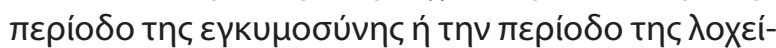

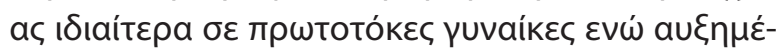

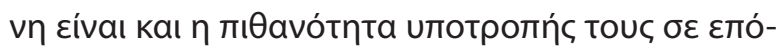

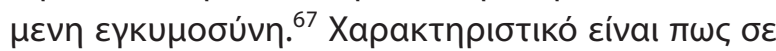

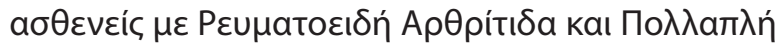
$\Sigma \kappa \lambda$ ń

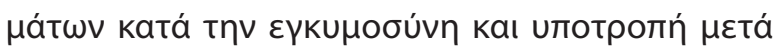
Tov TокहTó. ${ }^{69,70}$

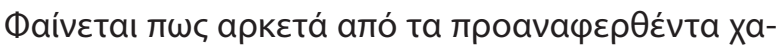

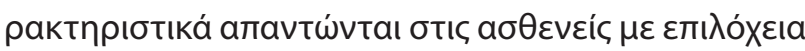

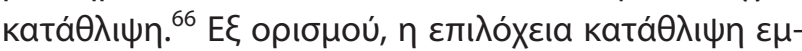

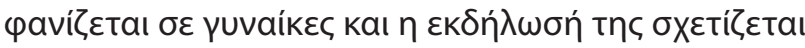

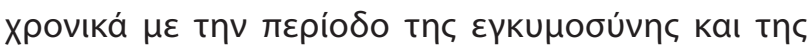

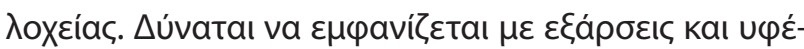

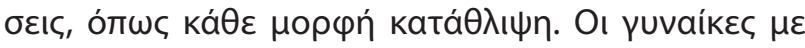

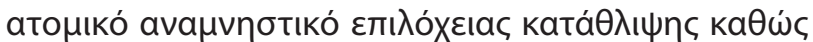

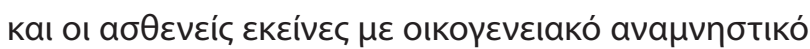

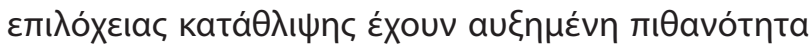

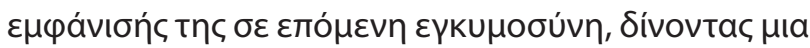

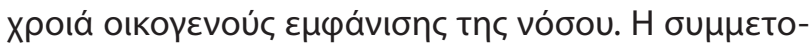

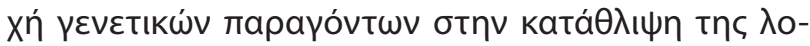

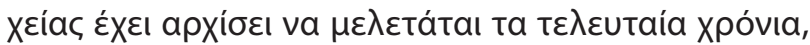

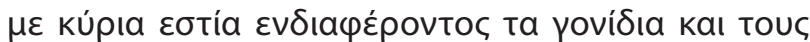

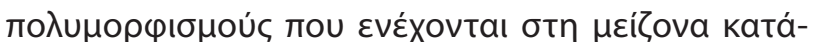

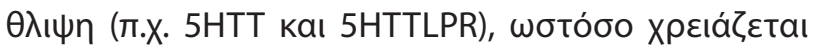

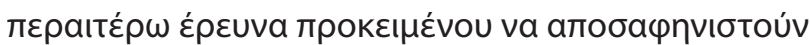

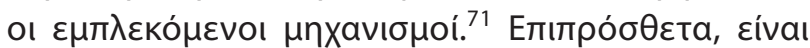

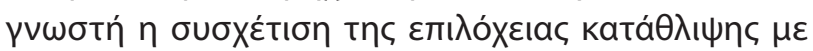

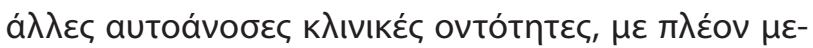

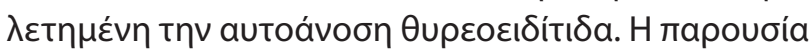

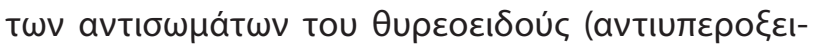

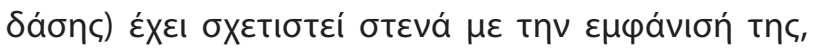

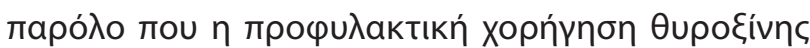

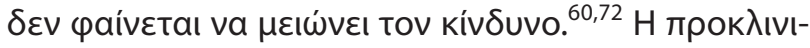

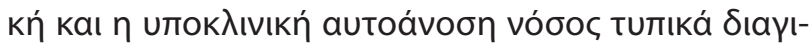

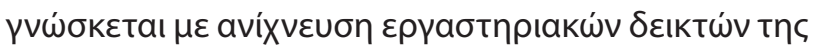

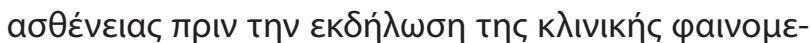

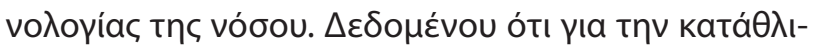

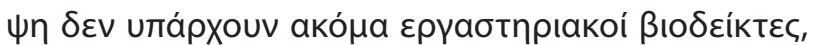

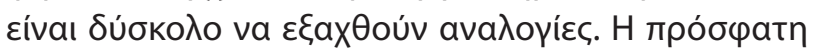

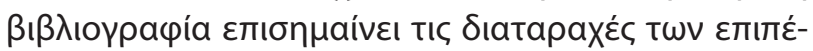

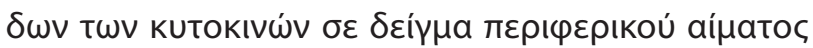

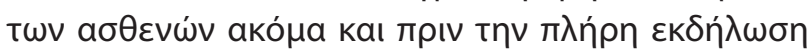

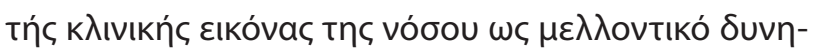

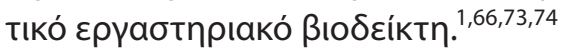

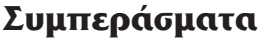

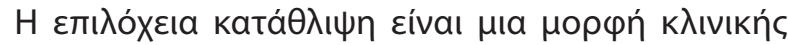

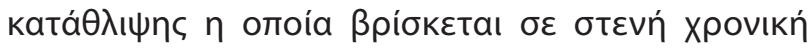

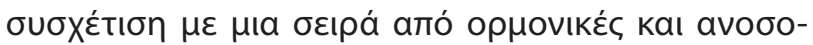

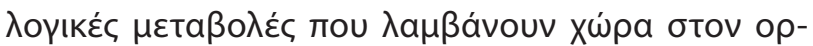

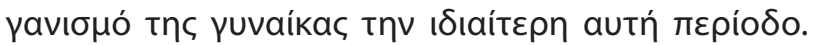

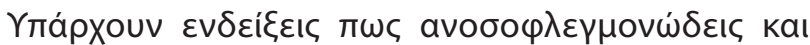

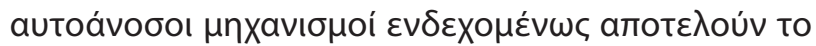

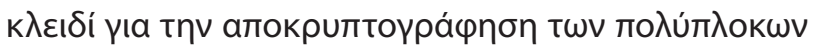

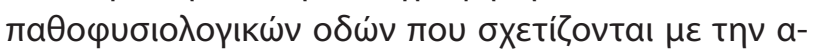

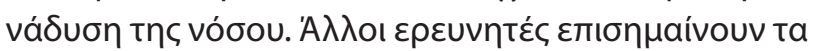

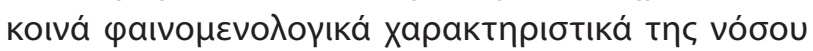

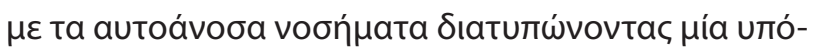

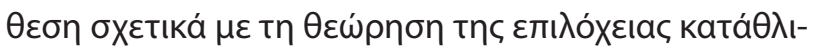

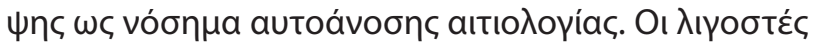

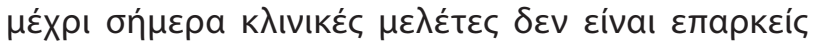




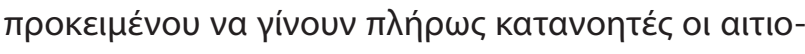
$\lambda$

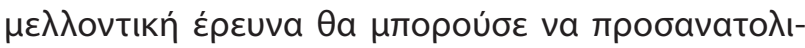

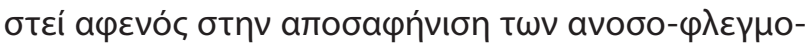

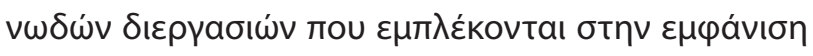

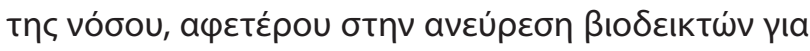

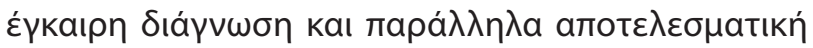

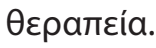

\title{
Immune-inflammatory and autoimmune mechanisms in postpartum depression
}

\author{
Ger. Konstantinou, ${ }^{1,2}$ A. Spyropoulou, ${ }^{1}$ I. Zervas ${ }^{1}$ \\ ${ }^{1} 1$ st Department of Psychiatry, Medical School, National and Kapodistrian University of Athens, Eginition Hospital, Athens, \\ ${ }^{2}$ Department of Psychiatry, 251 Hellenic Airforce V. A. General Hospital, Athens, Greece
}

Psychiatriki 2020, 31:47-56

Postpartum depression is a debilitating mental disorder with a high prevalence, usually related with a past psychiatric history of major depressive disorder, postpartum depression, bipolar disorder, premenstrual syndrome (PMS), and perinatal depressive symptoms during gestation. However, the existing literature does not sufficiently elucidate the pathophysiology of this clinical entity which appears in such a crucial period of woman's life. This review aims to search the available data regarding the involvement of immunological and autoimmune mechanisms in its onset. A literature review was conducted using web-based search engines provided by PubMed (for Medline database) and Google Scholar. Manuscripts in English and Greek language were included for the period 19902017. Nowadays, a large body of evidence indicates that depressive disorders are accompanied by activated neuro-immune, neuro-oxidative and neuro-nitrosative stress (IO\&NS) pathways. However clinical research regarding the biological mechanisms associated with PPD is a tough challenge as pregnancy and puerperium are periods of adaptive changes in pregnant women by definition. Two of the systems that have been studied as potentially contributing to the onset of PPD are: the activation of the Inflammatory Response System (IRS) and the deregulation of the Hypothalamic-PituitaryAdrenal axis (HPA). Controversial data indicate dysregulation of cytokines and other inflammatory agents in patients with PPD, as well as, a close correlation of immune-inflammatory mechanisms and kynurenine pathway. PPD has been closely associated with autoimmune diseases. It is notable that this entity shares many common traits with autoimmune diseases such as the genetic susceptibility, family history, the high correlation with other autoimmune diseases, clinical exacerbations and remissions, women's superiority in prevalence, and the possible re-occurrence during a future pregnancy. These facts suggest that the typical postpartum flare pattern, and other clinical characteristics, point towards an autoimmune etiology for PPD. There are indications that immune-inflammatory and autoimmune mechanisms may be the key to deciphering the complex pathophysiological pathways associated with the onset of PPD. Clinical studies have been insufficient to make clear the causative correlations of the underlying mechanisms involved. Future research could focus on the immune-inflammatory processes associated with the onset of the disease, as well as on potential biomarkers for an early diagnosis and an effective treatment of PPD.

Key words: Postpartum depression, immuno-inflammatory, stress, autoimmunity, cytokines, kynurenine, biomarkers. 


\section{Bıßהtoypacpía}

1. Maes M, Anderson G. Postpartum depression: psychoneuroimmunological underpinnings and treatment. Neuropsychiatr Dis Treatment 2013, 9:277-287, doi: 10.2147/ndt.s25320

2. Lancaster CA, Gold KJ, Flynn HA, Yoo H, Marcus SM, Davis MM. Risk factors for depressive symptoms during pregnancy: a systematic review. Am J Obstet Gynecol 2010, 202:5-14, doi: 10.1016/j.ajog.2009.09.007

3. Brummelte S, Galea LA. Depression during pregnancy and postpartum: contribution of stress and ovarian hormones. Prog Neuropsychopharmacol Biol Psychiatry 2010, 34:766-776, doi: 10.1016/j.pnpbp.2009.09.006

4. Halbreich U. Postpartum disorders: multiple interacting underlying mechanisms and risk factors. J Affect Disord 2005, 88:1-7, doi: 10.1016/j.jad.2005.05.002

5. O'Hara MW, Swain AM. Rates and risk of postpartum depression: a meta-analysis. Int Rev Psychiatry 1996, 8:37-54, doi: 10.3109/09540269609037816

6. Leonardou AA, Zervas YM, Papageorgiou CC, Marks MN, Tsartsara EC et al. Validation of the Edinburgh Postnatal Depression Scale and prevalence of postnatal depression at two months postpartum in a sample of Greek mothers. J Reprod Inf Psychol 2009, 27:28-39, doi: 10.1080/02646830802004909

7. Gonidakis F, Rabavilas AD, Varsou E, Kreatsas G, Christodoulou GN. A 6-month study of postpartum depression and related factors in Athens Greece. Compr Psychiatry 2008, 49:275-282, doi: 10.1016/j.comppsych.2007.05.018

8. Vivilaki VG, Dafermos V, Gevorgian L, Dimopoulou A, Patelarou E, Bick D et al. Validation of the Greek Maternal Adjustment and Maternal Attitudes Scale for Assessing Early Postpartum Adjustment. Women \& Health 2012, 52:369-390, doi: $10.1080 / 03630242.2012 .674089$

9. Koutra K, Vassilaki M, Georgiou V, Koutis A, Bitsios P, Kogevinas $M$ et al. Pregnancy, perinatal and postpartum complications as determinants of postpartum depression: the Rhea motherchild cohort in Crete, Greece. Epidemiol Psychiatr Sci 2018, 27):244-255, doi: 10.1017/S2045796016001062

10. Skalkidou A, Sylvén SM, Papadopoulos FC, Olovsson M, Larsson A, Sundstrom-Poromaa I. Risk of postpartum depression in association with serum leptin and interleukin-6 levels at delivery: A nested case - control study within the UPPSAT cohort. Psychoneuroendocrinology 2009, 34:1329-1337, doi: 10.1016/j.psyneuen.2009.04.003

11. Skrundz M, Bolten M, Nast I, Hellhammer DH, Meinlschmidt G. Plasma oyxtocin concentration during pregnancy is associated with development of postpartum depression. Neuropsychopharmacology 2011, 36:1886-1893, doi: 10.1038/ npp.2011.74

12. Groer MW, Morgan K. Immune, health and endocrine characteristics of depressed postpartum mothers. Psychoneuroendocrinology 2007, 32:133-139, doi: 10.1016/j.psyneuen.2006. 11.007

13. Meyer SE, Chrousos GP, Gold PW. Major depression and the stress system: a life span perspective. Dev Psychopathol 2001 13:565-580, doi: 10.1017/s095457940100308x
14. Miller AH, Maletic V, Raison CL. Inflammation and its discontents: the role of cytokines in the pathophysiology of major depression. Biol Psychiatry 2009, 65:732-741, doi: 10.1016/j. biopsych.2008.11.029

15. Dowlati Y, Herrmann N, Swardfager W, Liu H, Sham L, Reim EK et al. A meta-analysis of cytokines in major depression. Biol Psychiatry 2010, 67:446-457, doi: 10.1016/j.biopsych.2009. 09.033

16. Charmandari E, Tsigos C, Chrousos G. Endocrinology of the stress response. Annu Rev Physiol 2005, 67:259-284, doi: 10.1146/annurev.physiol.67.040403.120816

17. Elenkov IJ, Chrousos GP. Stress hormones, proinflammatory and anti-inflammatory cytokines, and autoimmunity. Ann NY Acad Sci 2002, 966:290, doi: 10.1111/j.1749-6632.2002. tb04229.x

18. Maes M, Ringel K, Kubera M, Berk M, Rybakowski J. Increased autoimmune activity against 5-HT: a key component of depression that is associated with inflammation and activation of cellmediated immunity, and with severity and staging of depression. J Affect Disord 2012, 136:386-392, doi: 10.1016/j.jad.2011. 11.016

19. Anisman H, Merali Z. Cytokines, stress and depressive illness: Brain-immune interactions. Ann Med 2003, 35:2, doi: 10.1080/ 07853890310004075

20. Chrousos GP, Gold PW. The concepts of stress and stress system disorders. JAMA 1992, 267:1244, doi: 10.1001/jama.1992. 03480090092034

21. Anisman H, Merali Z, Hayley S. Neurotransmitter, peptide and cytokine processes in relation to depressive disorder: Comorbidity between depression and neurodegenerative disorders. Progr Neurobiol 2008, 85:1-74, doi: 10.1016/j.pneurobio.2008.01.004

22. Maes M, Verkerk R, Bonaccorso S, Ombelet W, Bosmans E, Scharpé $S$. Depressive and anxiety symptoms in the early puerperium are related to increased degradation of tryptophan into kynurenine, a phenomenon which is related to immune activation. Life Sci 2002, 71:1837-1848, doi: 10.1016/s0024-3205 (02) 01853-2

23. Laugeray A, Launay JM, Callebert J, Surget A, Belzun C, Barone PR. Evidence for a key role of the peripheral kynurenine pathway in the modulation of anxiety- and depression-like behaviours in mice: focus on individual differences. Pharmacol Biochem Behav 2011, 98:161-168, doi: 10.1016/j.pbb.2010.12.008

24. Maes M, Rief W. Diagnostic classifications in depression and somatization should include biomarkers, such as disorders in the tryptophan catabolite (TRYCAT) pathway. Psychiatr Res 2012, 196:243-249, doi: 10.1016/j.psychres.2011.09.029

25. Pollak Y, Yirmiya R. Cytokine-induced changes in mood and behaviour: implications for 'depression due to a general medical condition', immunotherapy and antidepressive treatment. Int J Neuropsychopharmacol 2002, 5:389-399, doi: 10.1017/ s1461145702003152

26. Hines DJ, Choi HB, Hines RM, Phillips AG, MacVicar BA. Prevention of LPS-induced microglia activation, cytokine production and sickness behavior withTLR4 receptor interfering 
peptides. PLoS One 2013, 8:e60388, doi:10.1371/journal.pone. 0060388

27. Musselman DL, Nemeroff CB. Depression and endocrine disorders: focus on the thyroid and adrenal system. $B r J$ Psychiatry 1996, 30(Suppl):123-128, doi: 10.1192/s0007125000298504

28. Helmreich DL, Parfitt DB, Lu XY, Akil H, Watson SJ. Relation between the hypothalamic-pituitary-thyroid (HPT) axis and the hypothalamic-pituitary-adrenal (HPA) axis during repeated stress. Neuroendocrinology 2005, 81:183-92, doi: 10.1159/000087001

29. Robertson Blackmore E, Moynihan J, Rubinow D, Pressman E, Gilchrist M, O'Connor T. Psychiatric symptoms and proinflammatory cytokines in pregnancy. Psychosomat Med 2011, 73:656-663, doi: 10.1097/psy.0b013e31822fc277

30. Boufidou F, Lambrinoudaki I, Argeitis J, Zervas IM, Pliatsika P, Leonardou AA et al. CSF and plasma cytokines at delivery and postpartum mood disturbances. J Affect Disord 2009, 115:287-292, doi: 10.1016/j.jad.2008.07.008

31. Kim P, Strathearn L, Swain JE. The maternal brain and its plasticity in humans. Horm Behav 2016, 77:113-123, doi: 10.1016/j. yhbeh.2015.08.001

32. Yuan L, Liu S, Bai X, Gao Y, Liu G, Wang X et al. Oxytocin inhibits lipopolysaccharide-induced inflammation in microglial cells and attenuates microglial activation in lipopolysaccharidetreated mice. J Neuroinflammation 2016, 13:77, doi: 10.1186/ s12974-016-0541-7

33. Akman T, Akman L, Erbas O, Terek MC, Taskiran D, Ozsaran A. The preventive effect of oxytocin to Cisplatin-induced neurotoxicity: an experimental rat model. Biomed Res Int 2015, 2015:167235, doi: 10.1155/2015/167235

34. Bränn E, Papadopoulos F, Fransson E, White R, Edvinsson E, Hellgren $C$ et al. Inflammatory markers in late pregnancy in association with postpartum depression - A nested casecontrol study. Psychoneuroendocrinology 2017, 79:146-159, doi: 10.1016/j.psyneuen.2017.02.029

35. Mor G, Cardenas I, Abrahams V, Guller S. Inflammation and pregnancy: the role of the immune system at the implantation site. Ann N Y Acad Sci 2011, 1221:80-87, doi: 10.1111/j.17496632.2010.05938.x

36. Hannan NJ, Evans J, Salamonsen LA. Alternate roles for immune regulators: establishing endometrial receptivity for implantation. Expert Rev Clin Immunol 2011, 7:789-802, doi: 10.1586/eci. 11.65

37. Brewster JA, Orsi NM, Gopichandran N, McShane P, Ekbote UV, Walker JJ. Gestational effects on host inflammatory response in normal and pre-eclamptic pregnancies. Eur J Obstet Gynecol Reprod Biol 2008, 140:21-26, doi: 10.1016/j.ejogrb.2007.12.020

38. Dubicke A, Fransson E, Centini G, Andersson E, Byström B, Malmström A, et al. Pro-inflammatory and anti-inflammatory cytokines in human preterm and term cervical ripening. $J$ Reprod Immunol 2010, 84:176-185, doi: 10.1016/j.jri.2009.12.004

39. Malmström E, Sennström M, Holmberg A, Frielingsdorf $H$, Eklund $E$, Malmström $L$ et al. The importance of fibroblasts in remodelling of the human uterine cervix during pregnancy and parturition. Mol Hum Reprod 2007, 13:333-341, doi: 10.1093/ molehr/gal117

40. Sennström MB, Ekman G, Westergren-Thorsson G, Malmström A, Byström B, Endrésen $U$ et al. Human cervical ripening, an inflammatory process mediated by cytokines. Mol Hum Reprod 2000, 6:375-381, doi: 10.1093/molehr/6.4.375

41. Fransson E, Dubicke A, Byström B, Ekman-Ordeberg G, Hjelmstedt $A$, Lekander $M$. Negative emotions and cytokines in maternal and cord serum at preterm birth. Am J Reprod Immunol 2011, 67:506-514, doi: 10.1111/j.1600-0897.2011.01081.x

42. Roomruangwong C, Anderson G, Berk M, Stoyanov D, Carvalho AF, Maes M. A neuro-immune, neuro-oxidative and neuro-nitrosative model of prenatal and postpartum depression. Prog Neuropsychopharmacol Biol Psychiatry 2018, 81:262-274, doi: 10.1016/j.pnpbp.2017.09.015

43. Roomruangwong C, Kanchanatawan B, Sirivichayakul S, Mahieu B, Nowak G, Maes M. Lower serum zinc and higher CRP strongly predict prenatal depression and physiosomatic symptoms, which all together predict postnatal depressive symptoms. Mol Neurobiol 2016, 54:1500-1512, doi: 10.1007/ s12035-016-9741-5

44. Maes M, Ombelet W, Verkerk R, Bosmans E, Scharpé S. Effects of pregnancy and delivery on the availability of plasma tryptophan to the brain: relationships to delivery-induced immune activation and early post-partum anxiety and depression. Psychol Med 2001, 31:847-858, doi: 10.1017/s0033291701004007

45. Skalkidou A, Hellgren C, Comasco E, Sylven S, Sundstrom Poromaa I. Biological aspects of postpartum depression. Womens Health (Lond) 2012, 8:659-672, doi: 10.2217/whe.12.55

46. Osborne LM, Monk, C. Perinatal depression-the fourth inflammatory morbidity of pregnancy? Theory and literature review. Psychoneuroendocrinology 2013, 38:1929-1952, doi: 10.1016/j. psyneuen.2013.03.019

47. Corwin EJ, Pager K. The psychoneuroimmunology of postpartum depression. J Womens Health 2008, 17:1529-1534, doi: 10.1089/jwh.2007.0725

48. Okun ML, Luther J, Prather AA, Perel JM, Wisniewski S, Wisner $\mathrm{K}$. Changes in sleep quality but not hormones predict time to postpartum depression recurrence. J Affect Disord 2011, 130:378-384, doi: 10.1016/j.jad.2010.07.015

49. Chrousos GP. The hypothalamic-pituitary-adrenal axis and immune-mediated inflammation. N Engl J Med 1995, 332:1351, doi: 10.1056/nejm199505183322008

50. Yim IS, Glynn LM, Dunkel-Schetter C, Hobel CJ, Chicz-DeMet A, Sandman CA. Risk of postpartum depressive symptoms with elevated corticotropin-releasing hormone in human pregnancy. Arch Gen Psychiatry 2009, 66:162-169, doi: 10.1001/ archgenpsychiatry.2008.533

51. Raison CL, Capuron L, Miller AH. Cytokines sing the blues: inflammation and the pathogenesis of depression. Trends Immunol 2006, 27:24-31, doi: 10.1016/j.it.2005.11.006

52. Magiakou MA, Mastorakos G, Rabin D, Dubbert B, Gold PW, Chrousos GP. Hypothalamic corticotropin-releasing hormone suppression during the postpartum period: Implications for the increase in psychiatric manifestations at this time. $J$ Clin Endocrinol Metab 1996, 81:1912, doi: 10.1210/jcem.81.5. 8626857

53. Nielsen FD, Videbech P, Hedegaard M, Dalby SJ, Secher NJ. Postpartum depression: Identification of women at risk. $\mathrm{Br} \mathrm{J}$ Obstetr Gynaecol 2000, 107:1210-1217, doi: 10.1111/j.1471-0528. 2000.tb11609.x 
54. Skalkidou A, Sylven SM, Papadopoulos FC, Olovsson M, Larsson A, Sundstrom-Poromaa I. Risk of postpartum depression in association with serum leptin and interleukin-6 levels at delivery: a nested case-control study within the UPPSAT cohort. Psychoneuroendocrinology 2009, 34:1329-1337, doi: 10.1016/j. psyneuen.2009.04.003

55. Corwin EJ, Pajer K, Paul S, Lowe N, Weber M, McCarthy DO. Bidirectional psychoneuroimmune interactions in the early postpartum period influence risk of postpartum depression. Brain Behav Immun 2015, 49:86-93, doi: 10.1016/j.bbi.2015.04.012

56. Krause D, Jobst A, Kirchberg F, Kieper S, Härtl K, Kästner R et al. Prenatal immunologic predictors of postpartum depressive symptoms: a prospective study for potential diagnostic markers. Eur Arch Psychiatry Clin Neurosci 2014, 264:615-624, doi: 10.1007/s00406-014-0494-8

57. Le Donnel M, Mento C, Settineri S, Antonelli A, Benvenga S. Postpartum mood disorders and thyroid autoimmunity. Front Endocrinol 2017, 8:91, doi: 10.3389/fendo.2017.00091

58. Bloch M, Daly RC, Rubinow DR. Endocrine factors in the etiology of postpartum depression. Compr Psychiatry 2003, 44:234-246, doi: 10.1016/s0010-440x(03)00034-8

59. Carter CS, Altemus M, Chrousos GP. Neuroendocrine and emotional changes in the post-partum period. Prog Brain Res 2001, 133:241-249, doi: 10.1016/s0079-6123(01)33018-2

60. Davies TF. The thyroid immunology of the postpartum period. Thyroid 1999, 9:675-84, doi: 10.1089/thy.1999.9.675

61. Lucas A, Pizzaro E, Granada ML, Salinas I, Sanmarti A. Postpartum thyroid dysfunction and postpartum depression: are they linked disorders? Clin Endocrinol (Oxf) 2001, 55:809-814, doi: 10.1046/j.1365-2265.2001.01421.x

62. Raghupathy R, Makhseed M, Azizieh F, Omu A, Gupta M, Farhat R. Cytokine production by maternal lymphocytes during normal human pregnancy and in unexplained recurrent spontaneous abortion. Hum Reprod 2000, 15:713-718, doi: 10.1093/hum$\mathrm{rep} / 15.3 .713$

63. Marzi M, Vigano A, Trabattoni D, Villa ML, Salvaggio A, Clerici $\mathrm{E}$ et al. Characterization of type 1 and type 2 cytokine production profile in physiologic and pathologic human pregnancy. Clin Exp Immunol 1996, 106:127-133, doi: 10.1046/j.13652249.1996.d01-809.x

64. Sliwa K, Fett J, Elkayam U. Peripartum cardiomyopathy. Lancet 2006, 368:687-693, doi: 10.1016/s0140-6736(06)69253-2

65. Schramm C, Herkel J, Beuers U, Kanzler S, Galle PR, Lohse AW. Pregnancy in autoimmune hepatitis: outcome and risk factors. Am J Gastroenterol 2006, 101:556-560, doi: 10.1111/j.1572-0241. 2006.00479.x
66. Gleicher N. Postpartum depression, an autoimmune disease? Autoimmun Rev 2007, 6:572-576, doi: 10.1016/j.autrev.2007. 04.002

67. Davidson A, Diamond B. Autoimmune diseases. N Engl J Med 2001, 345:340-350, doi: 10.1056/nejm200108023450506

68. Gleicher N, Barad DH. Gender as risk factor for autoimmune diseases. J Autoimmunity 2007, 28:1-6, doi: 10.1016/j. jaut.2006.12.004

69. Hazes JM, Coulie PG, Geenen V, Vermeire S, Carbonnel F, Louis $E$ et al. Rheumatoid arthritis and pregnancy: evolution of disease activity and pathophysiological considerations for drug use. Rheumatology (Oxford) 2011, 50:1955-1968, doi: 10.1093/ rheumatology/ker302

70. Voskuhl R, Momtazee C. Pregnancy: Effect on Multiple Sclerosis, Treatment Considerations, and Breastfeeding. Neurotherapeutics 2017, 14:974-984, doi: 10.1007/s13311-017-0562-7

71. Couto TC, Brancaglion MY, Alvim-Soares A, Moreira L, Garcia FD, Nicolato $R$ et al. Postpartum depression: A systematic review of the genetics involved. World J Psychiatry 2015, 5:103111, doi: 10.5498/wjp.v5.i1.103

72. Sylvin SM, Elenis E, Michelakos T. Thyroid function tests at delivery and risk for postpartum depressive symptoms. Psychoneuroendocrinology 2013, 38:1007-1013, doi: 10.1016/j. psyneuen.2012.10.004

73. Salel E, El-Bahei W, El-Hadidy M, Zayed A. Predictors of postpartum depression in a sample of Egyptian women. Neuropsychiatric Dis Treat 2013, 9:15-24, doi: 10.2147/NDT.S37156

74. Serati M, Redaelli M, Buoli M, Altamura AC. Perinatal Major Depression Biomarkers: A systematic review. J Affect Disord 2016, 193:391-404, doi: 10.1016/j.jad.2016.01.027

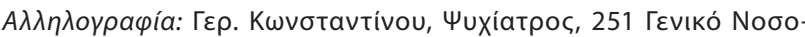

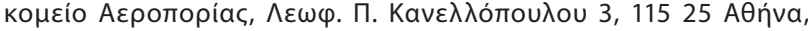
$\operatorname{T\eta \lambda :} 6983519$ 996, e-mail: gerkonst@gmail.com 


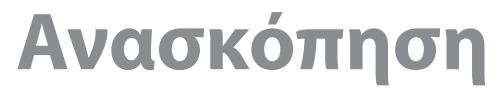 Review
}

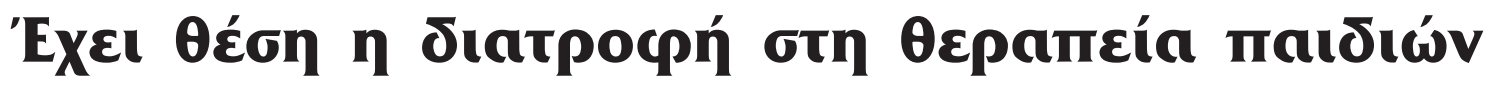

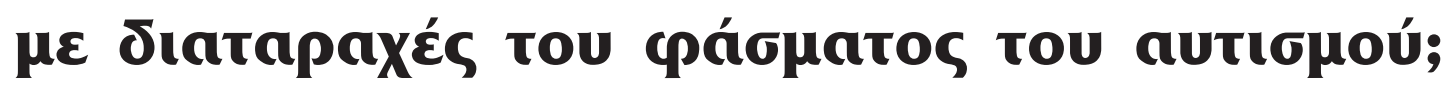

\author{
M. Гஸ́үou, ${ }^{1}$ Г. Koגıó $\varsigma^{2}$

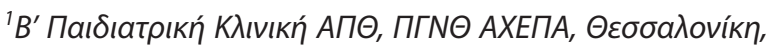

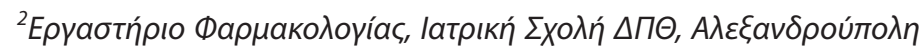 \\ Uuxıатрıкń 2020, 31:57-69
}

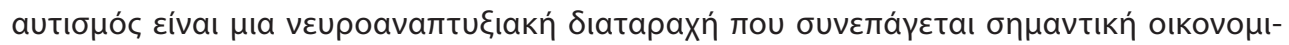

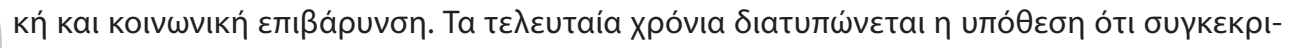

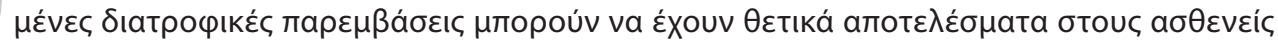

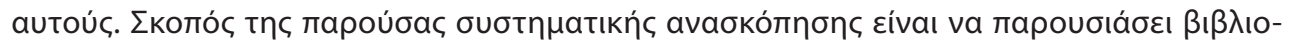

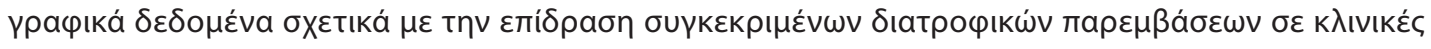

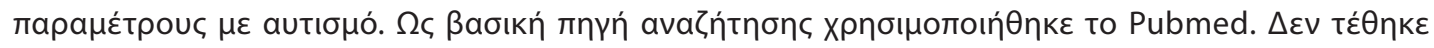

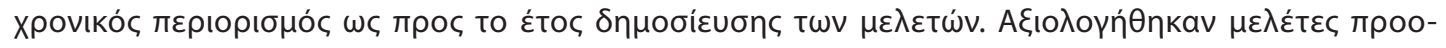

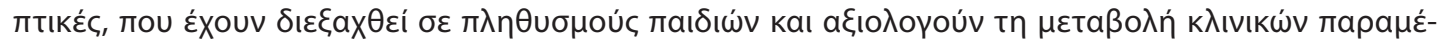

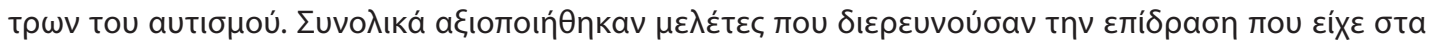

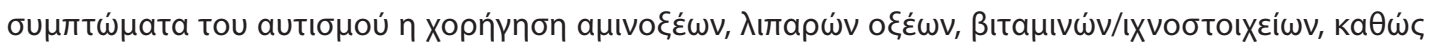

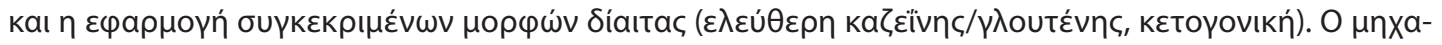

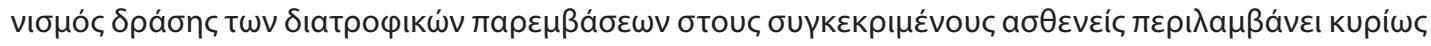

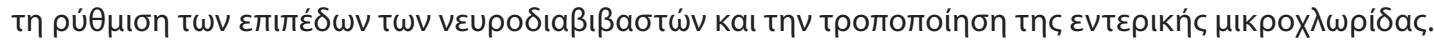

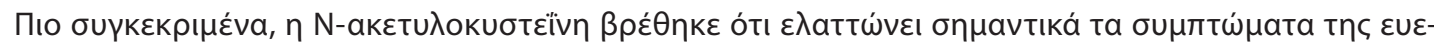

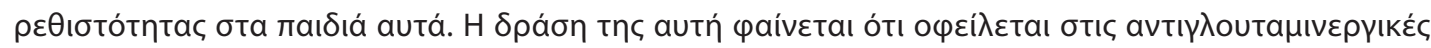

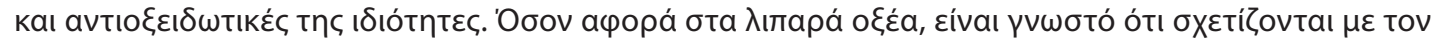

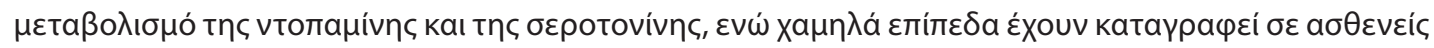

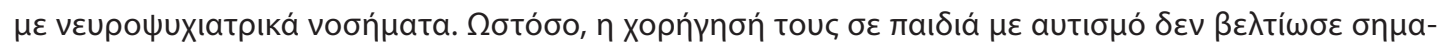

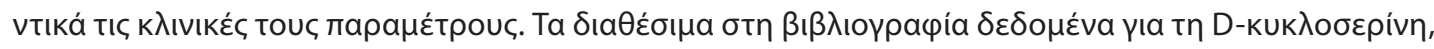

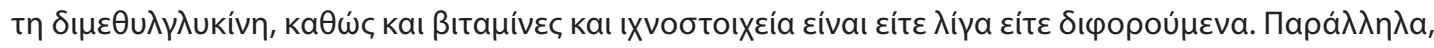

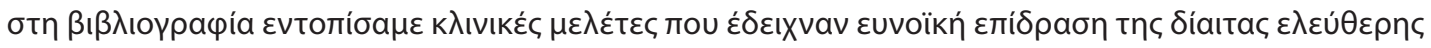

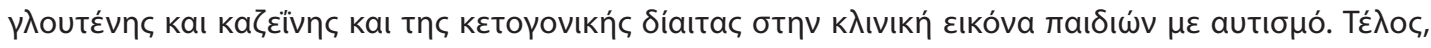




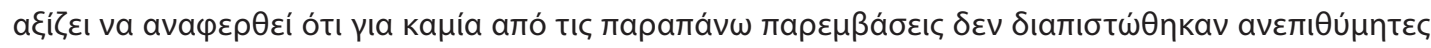

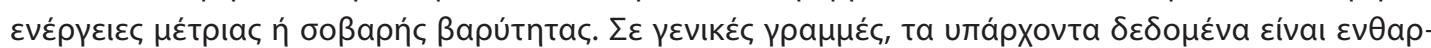

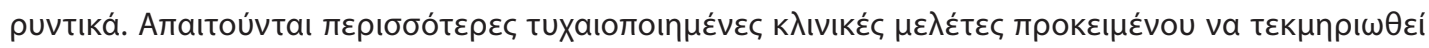

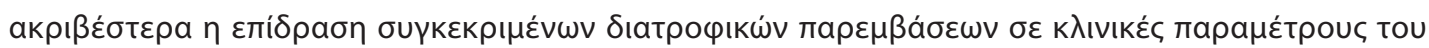
autiouoú.

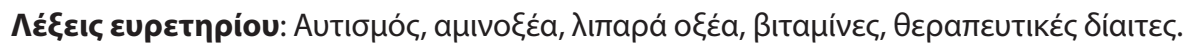

\section{Eıбaywyń}

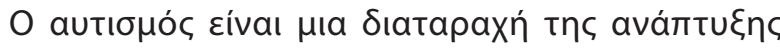

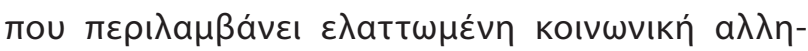

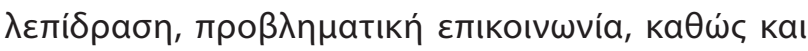

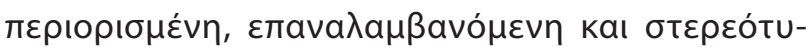

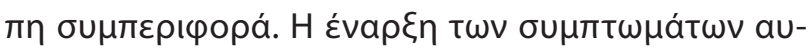

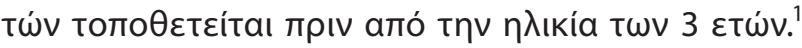

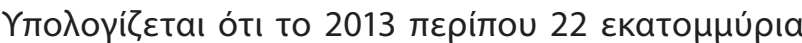

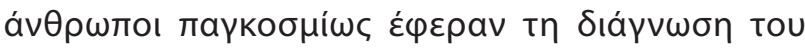

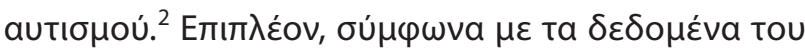

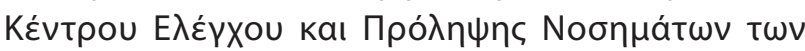

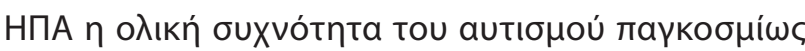

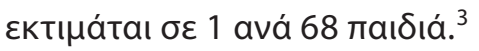

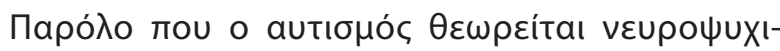

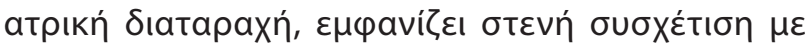

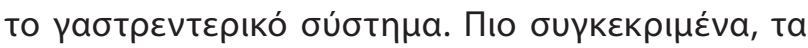

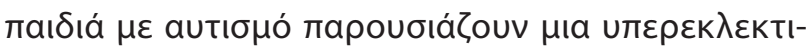

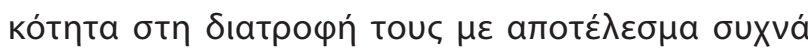

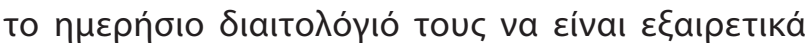

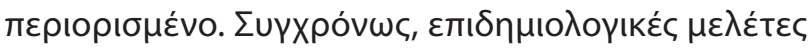

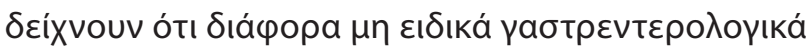

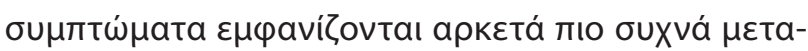

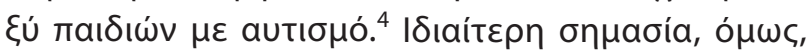

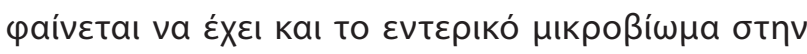

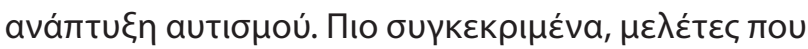

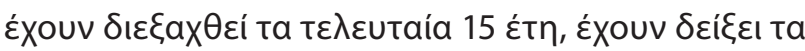

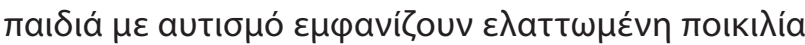

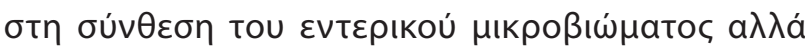

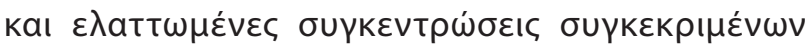

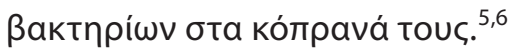

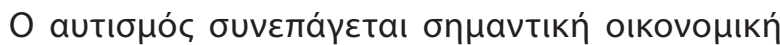

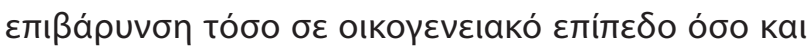

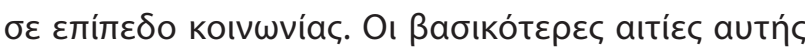

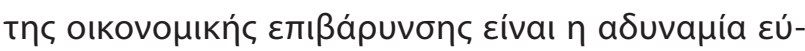

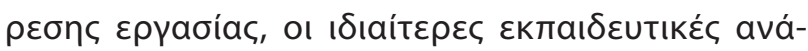

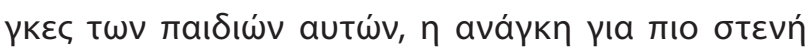

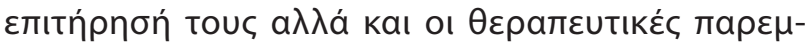

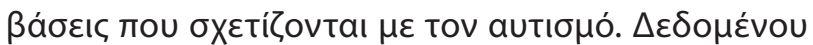

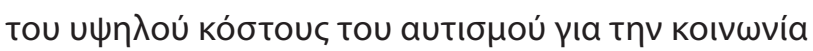

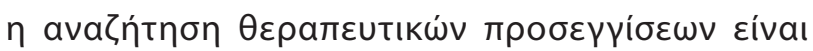

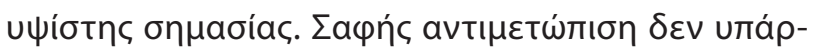

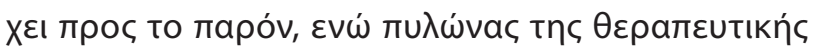

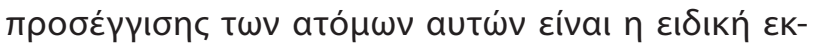

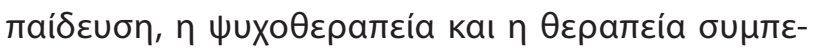

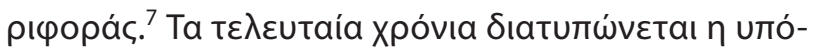

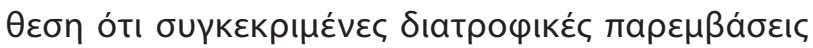

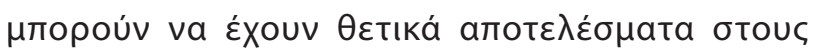

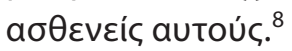

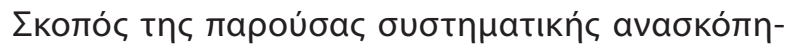

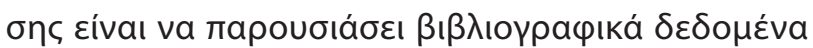

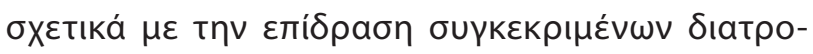

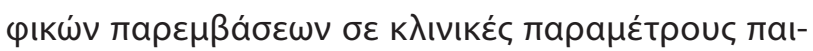

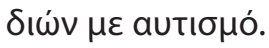

\section{MéӨodos}

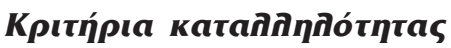

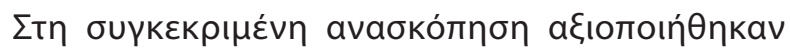

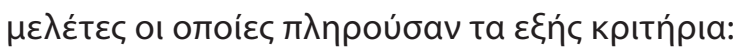

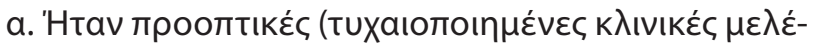

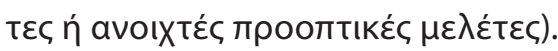

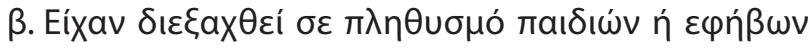

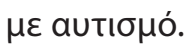

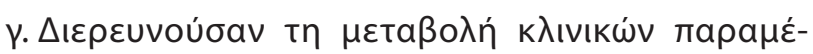

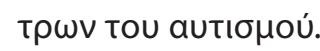

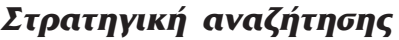

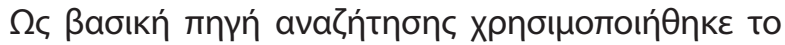

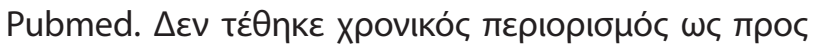

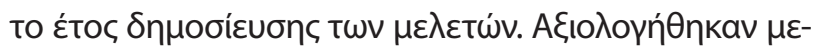

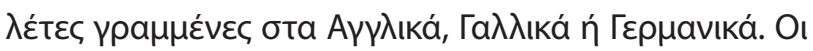

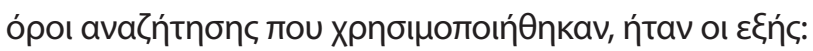
"autism", "vitamins", "minerals", "aminoacids", "diet", "carnitine", "probiotics", "prebiotics", "fattyacids", "nutritional interventions", "dietary interventions", "diets". 
А

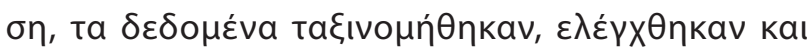

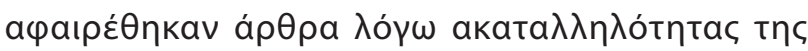

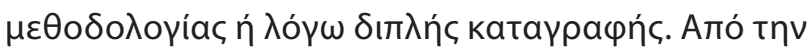

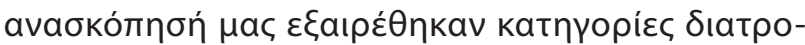

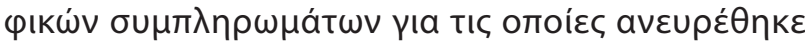

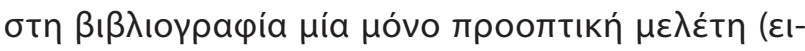
kóva 1).

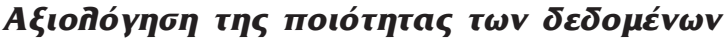

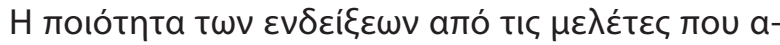

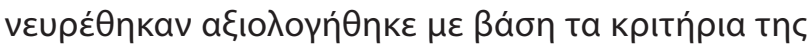

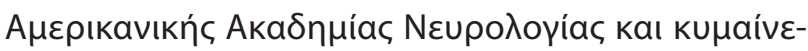

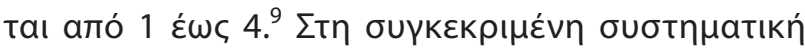

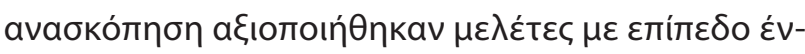

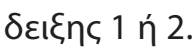

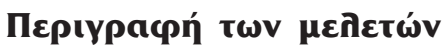

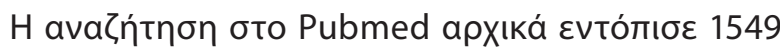

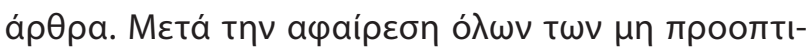

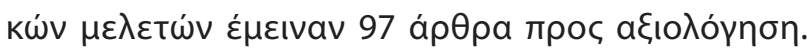

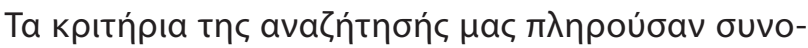

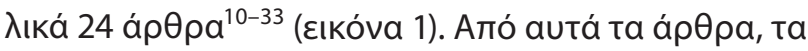

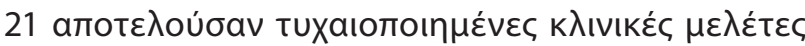

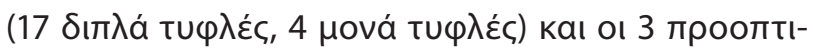

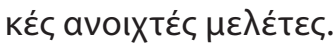

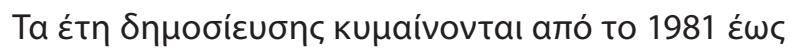

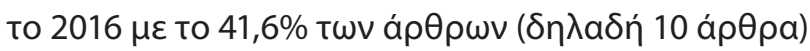

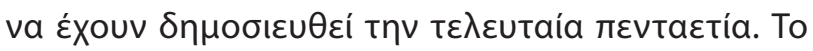

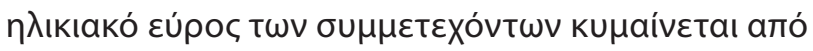

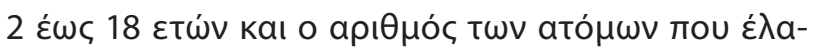

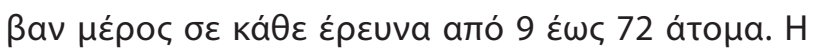

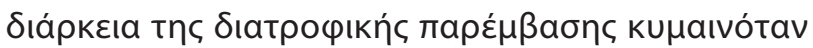

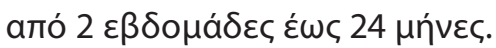

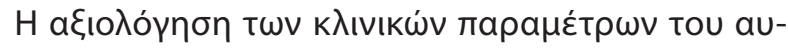

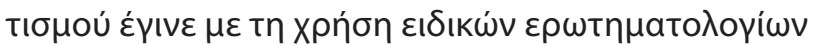

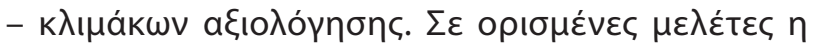

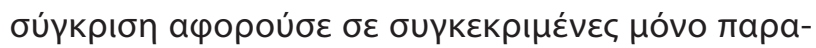

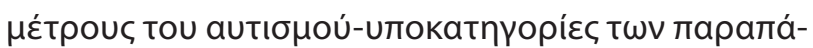

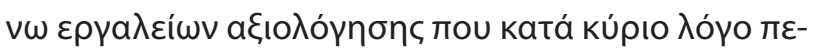

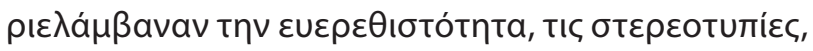

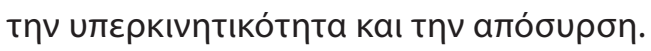

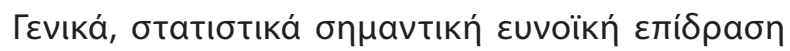

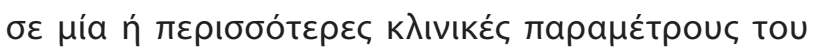

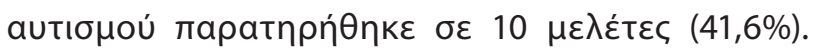

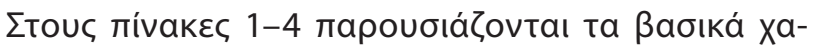

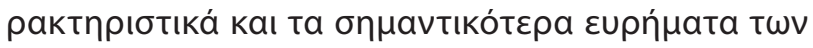

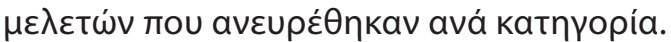

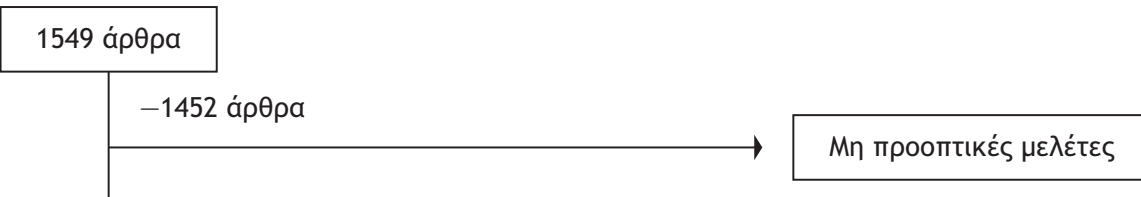

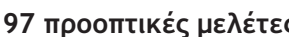

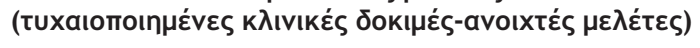

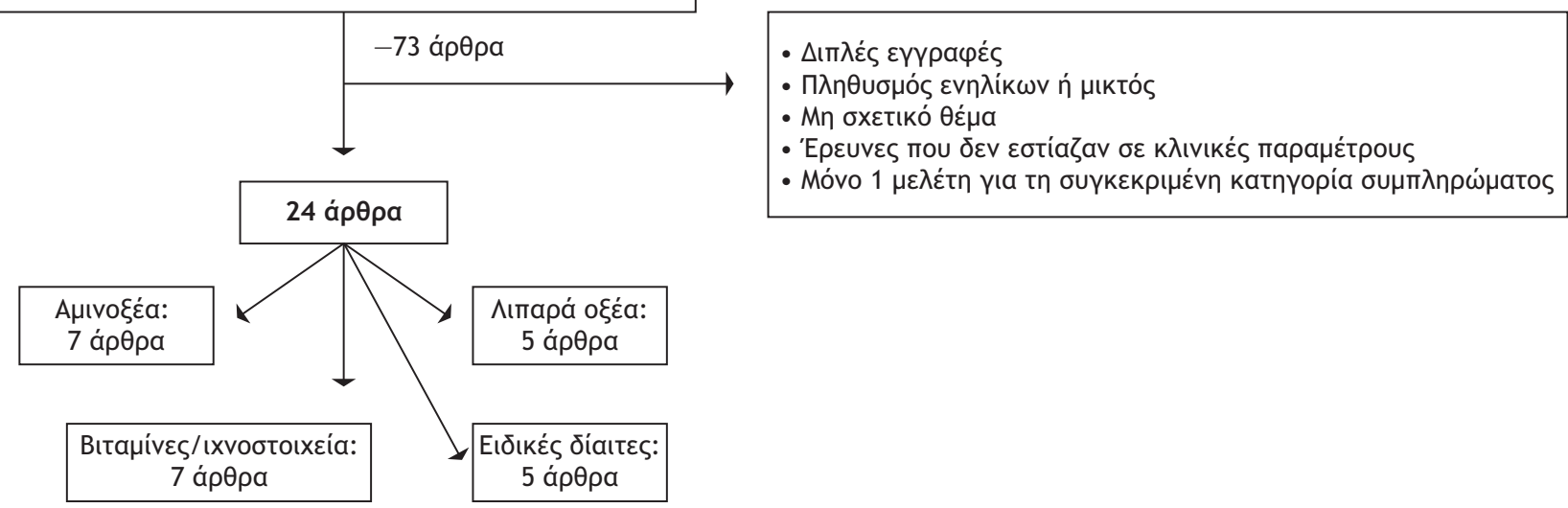

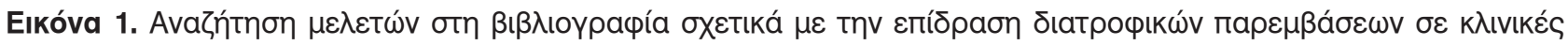

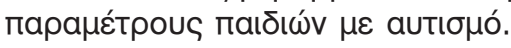




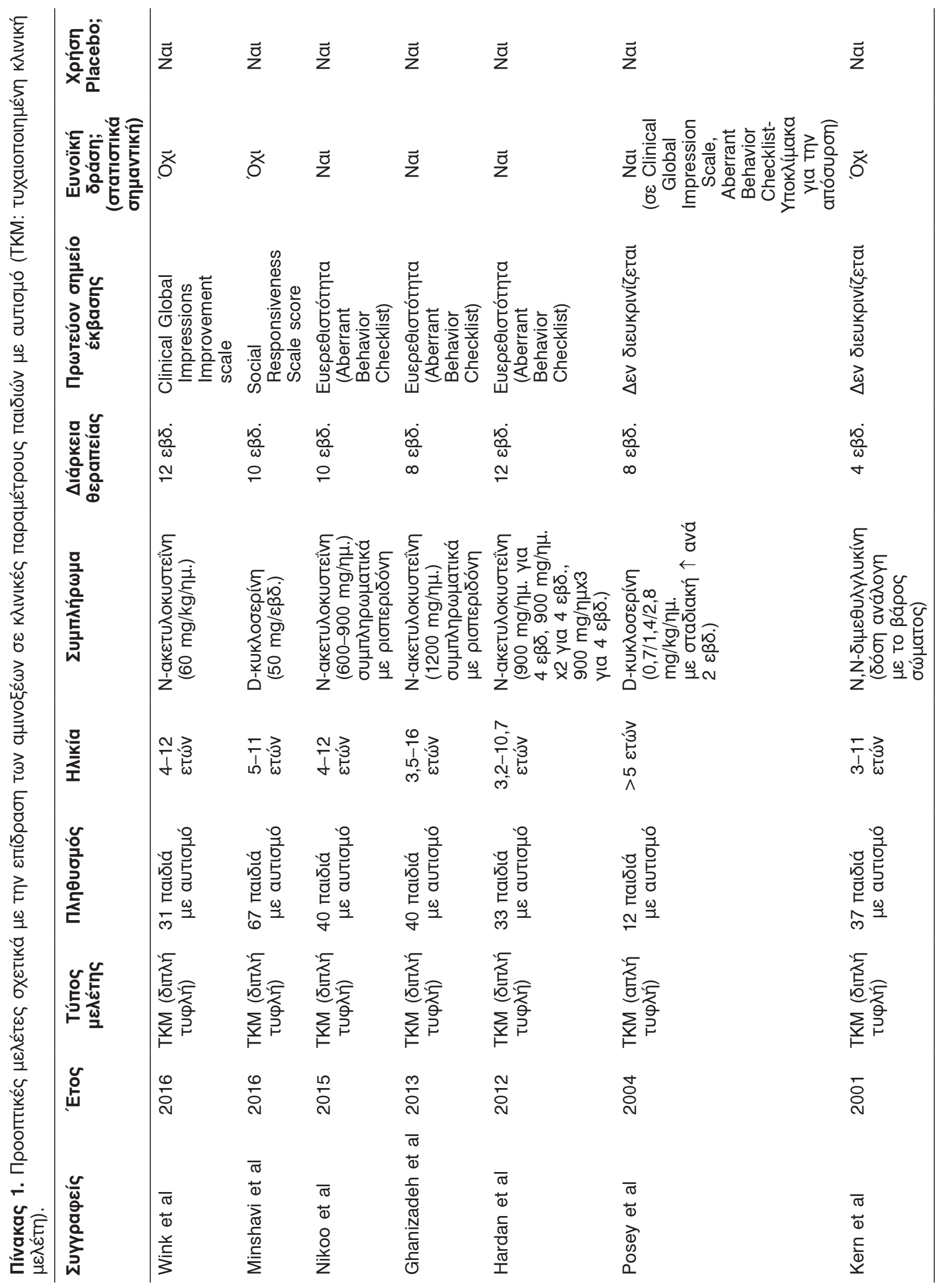




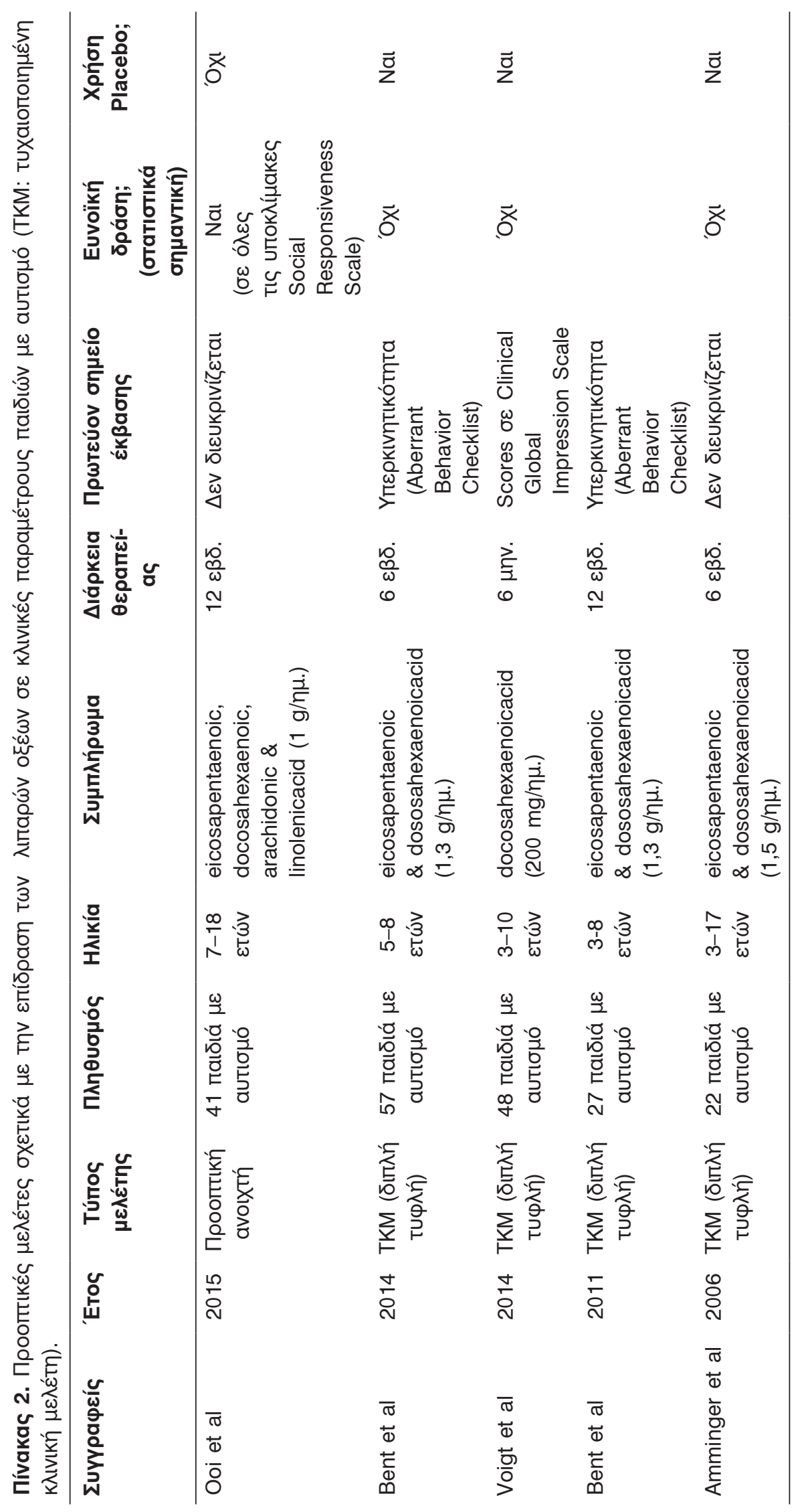









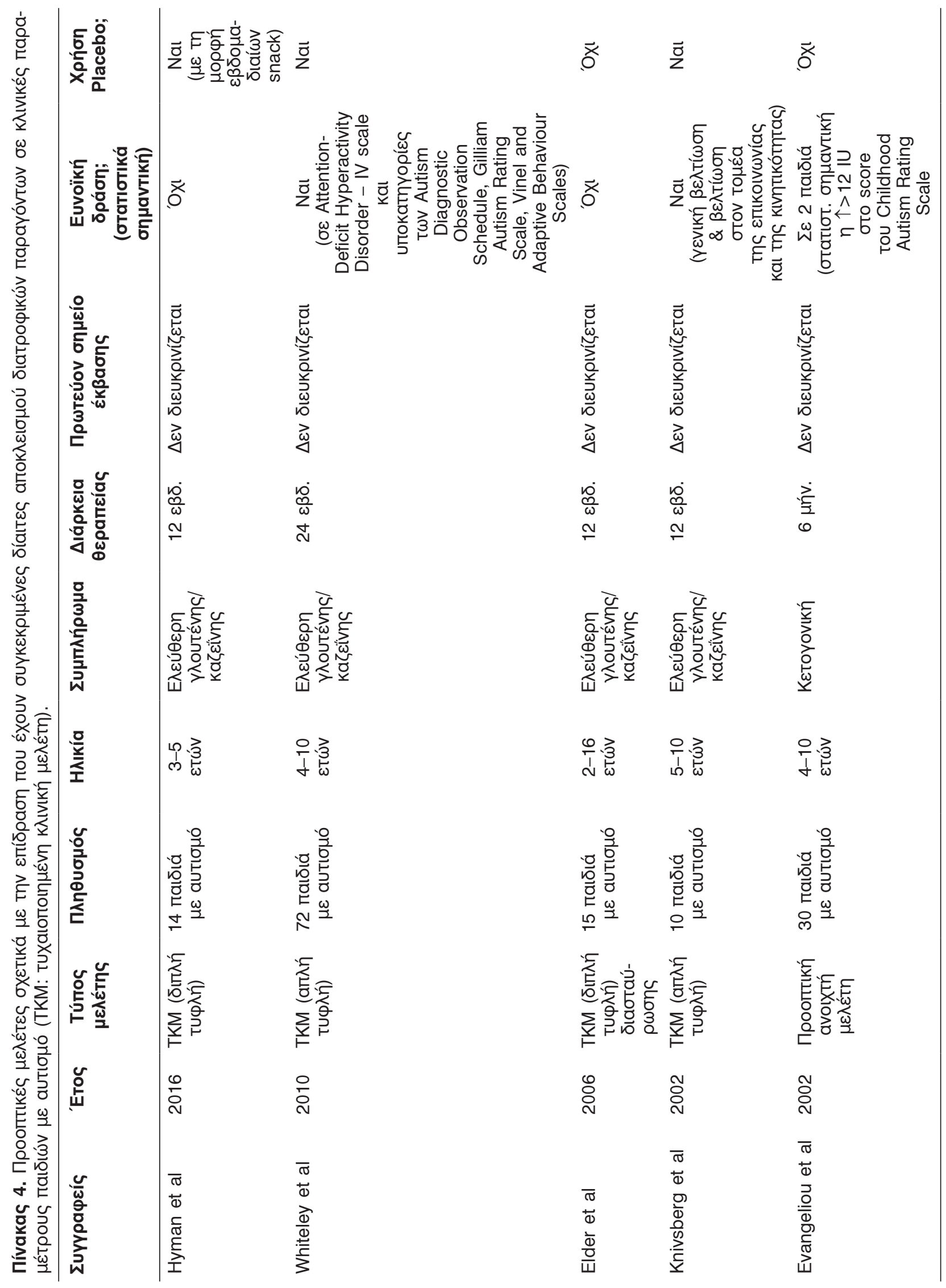




\section{Aptvoß́́a}

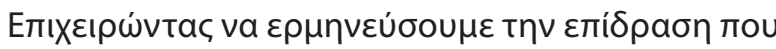

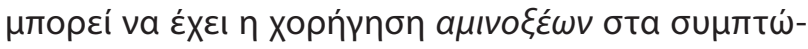

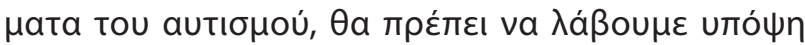

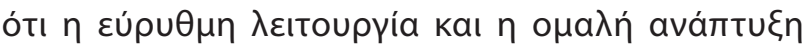

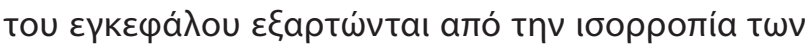

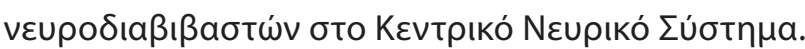

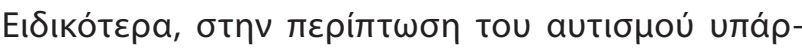

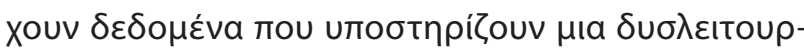

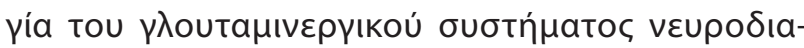

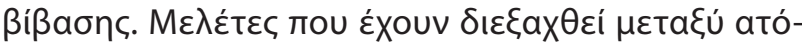

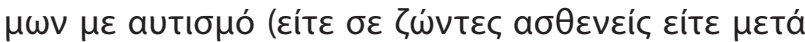

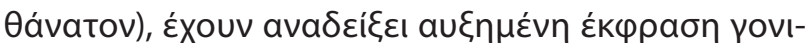

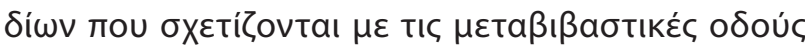

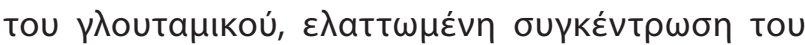

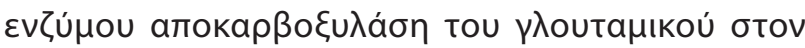

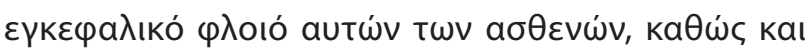

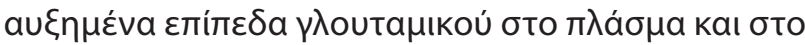

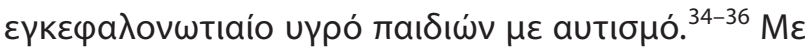

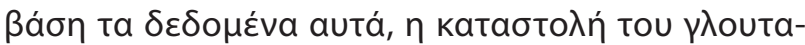

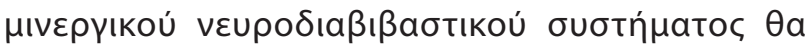

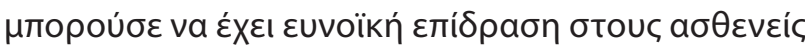

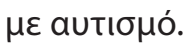

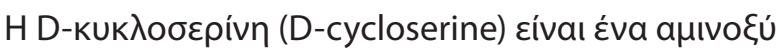

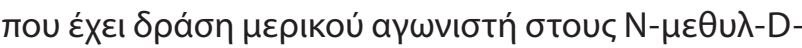

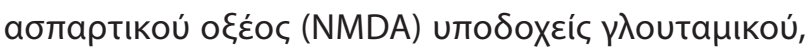

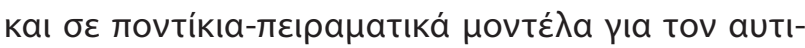

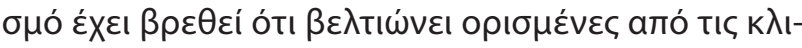

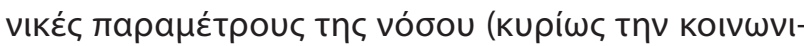

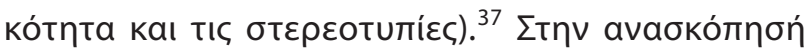

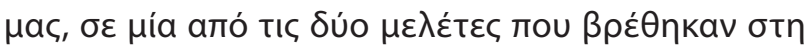

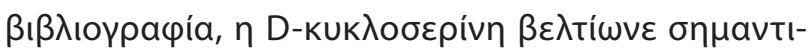

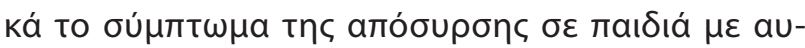
тıбнó.

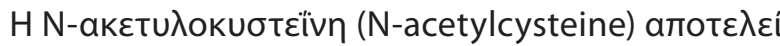

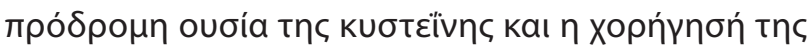

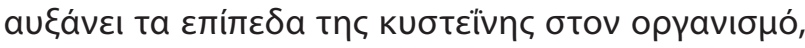

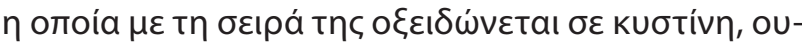

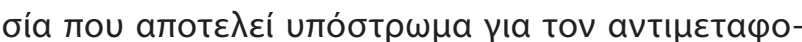

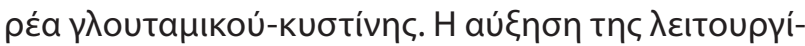

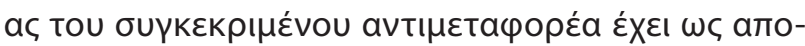

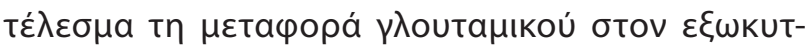

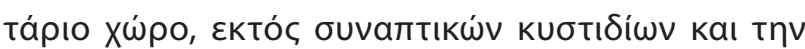

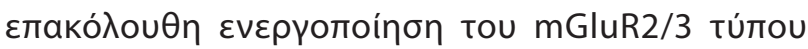

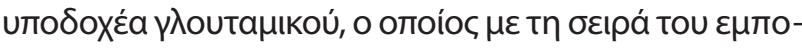

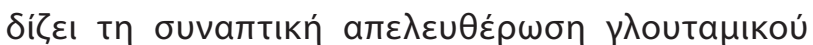

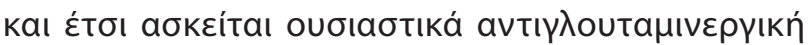

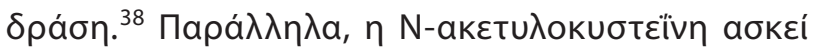

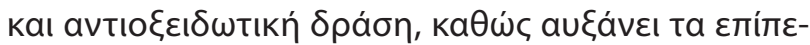

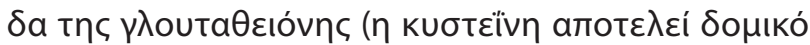

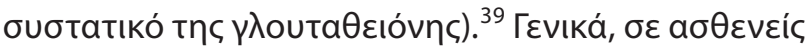

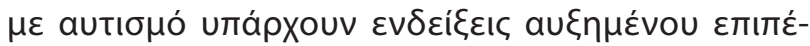

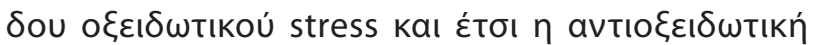

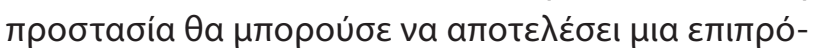

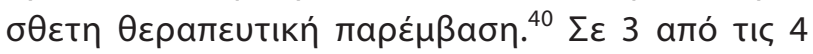

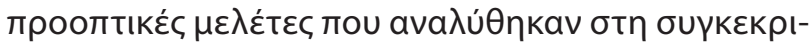

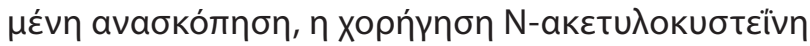

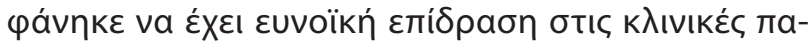

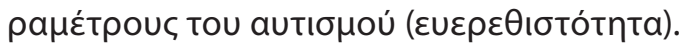

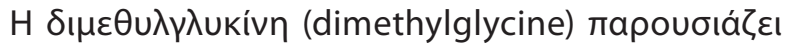

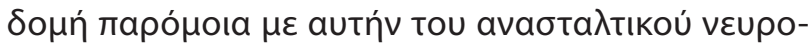

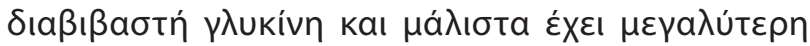

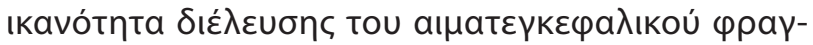

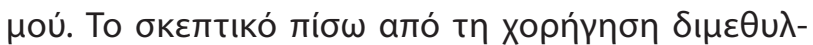

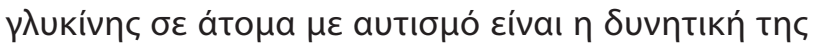

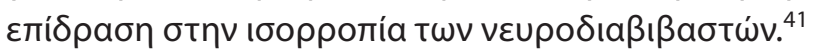

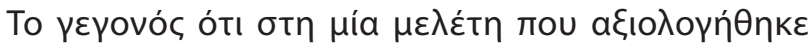

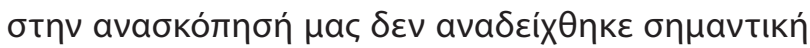

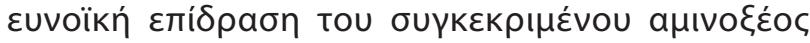

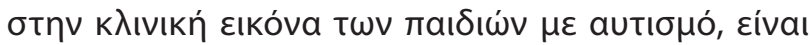

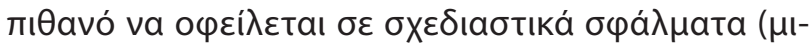

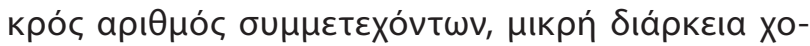

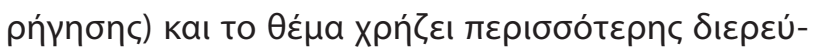

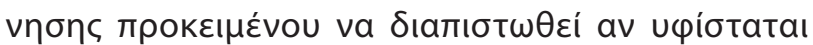

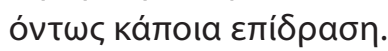

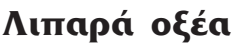

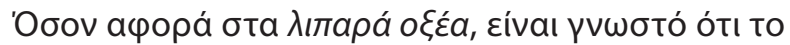

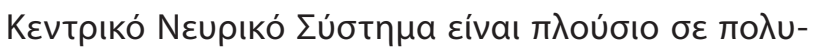

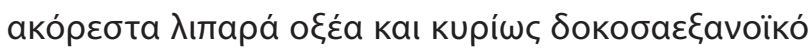

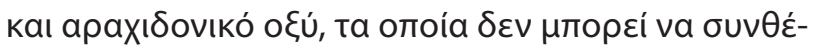

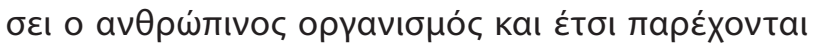

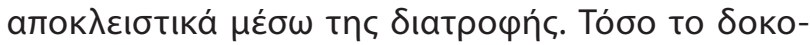

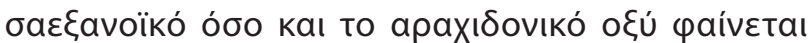

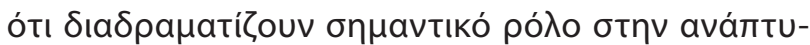

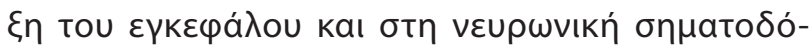

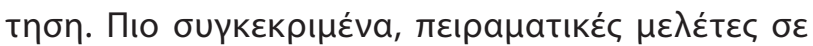

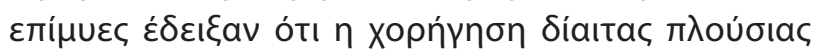

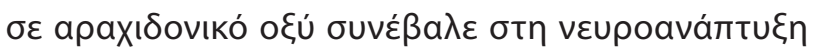

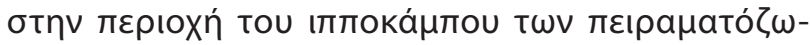

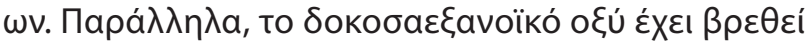




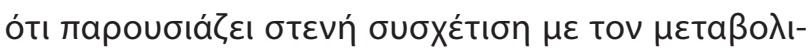

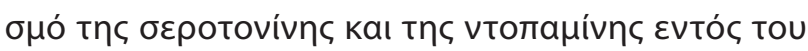

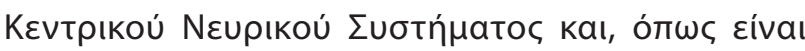

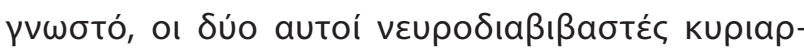

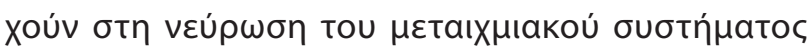

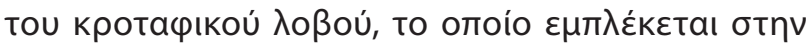

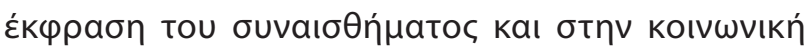
$a \lambda \lambda \eta \lambda \varepsilon \pi i \delta$ paon. ${ }^{42-44}$

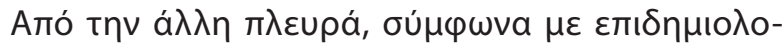

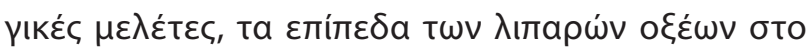

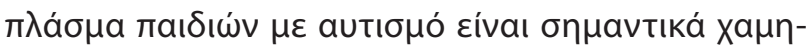

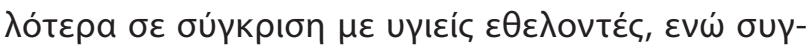

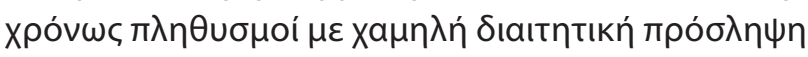

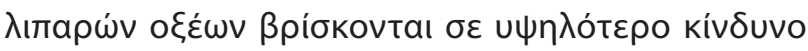

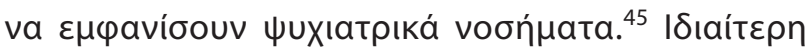

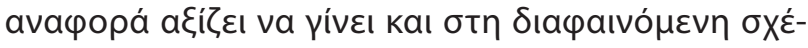

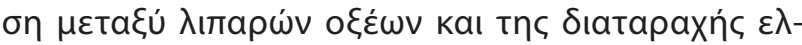

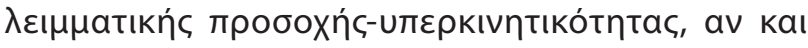

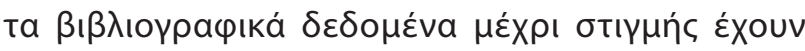

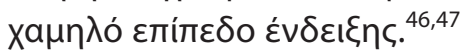

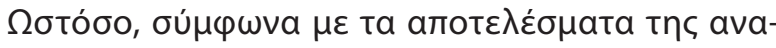

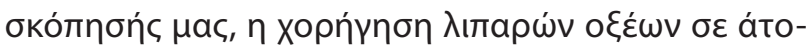

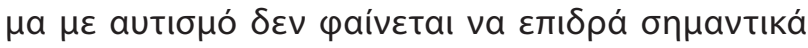

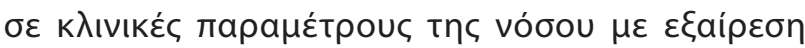

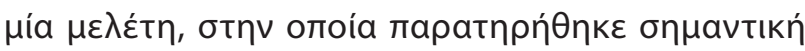

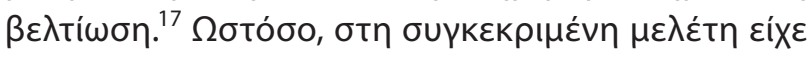

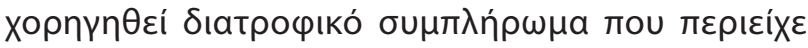

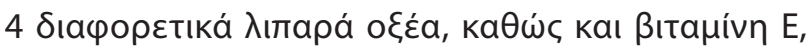

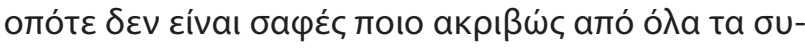

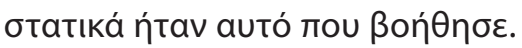

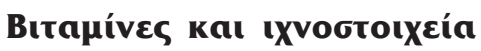

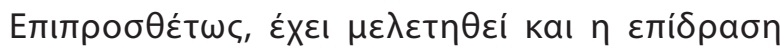

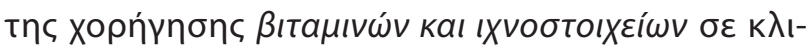

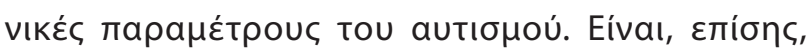

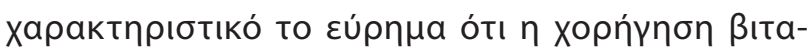

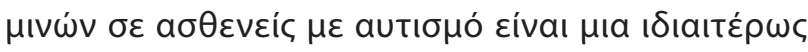

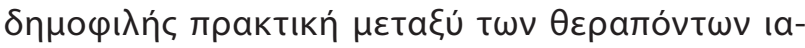

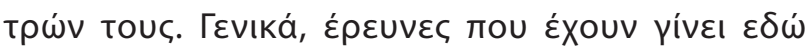

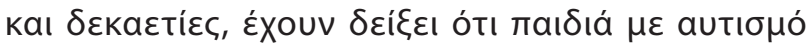

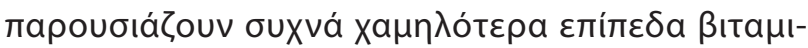

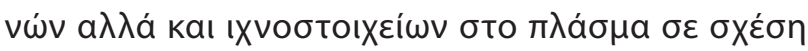

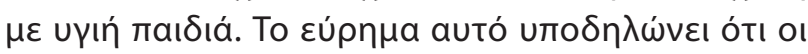

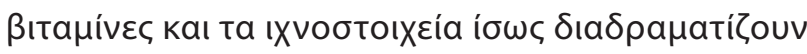

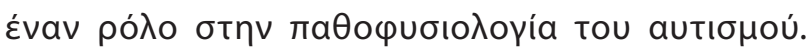

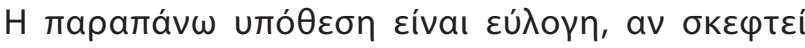

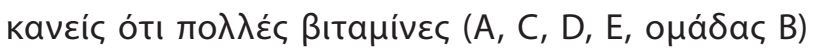
kaı ıxvootoıx

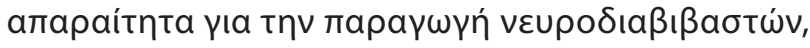

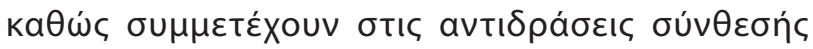

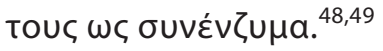

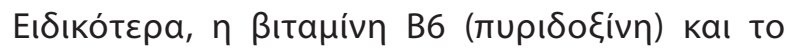

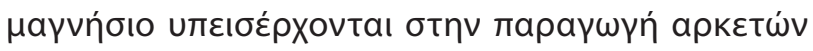

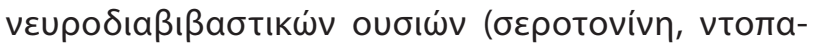

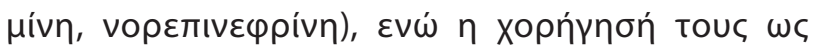

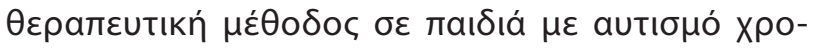

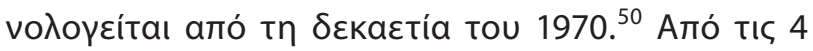

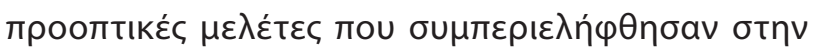

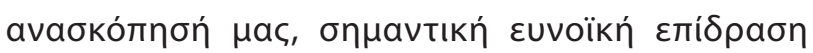

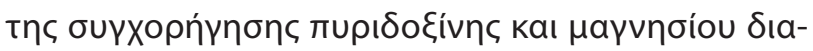

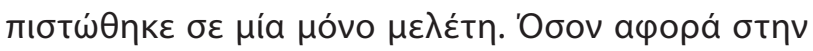

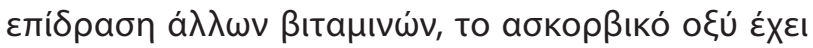

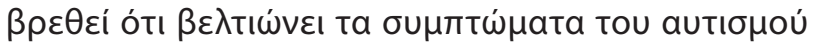

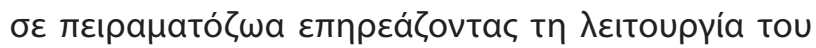

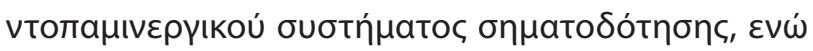

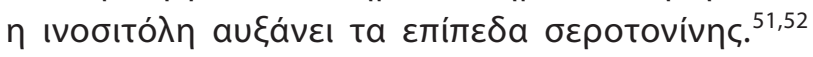

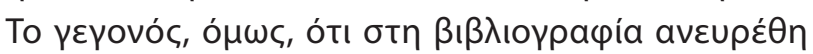

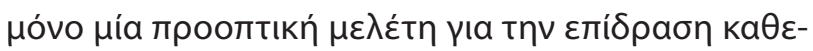

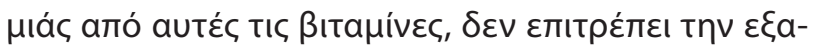

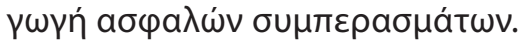

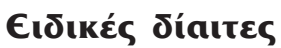

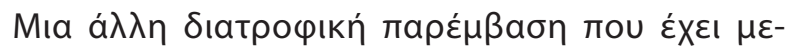

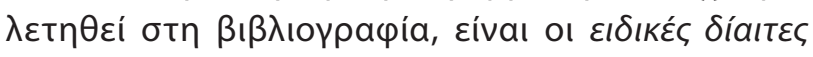

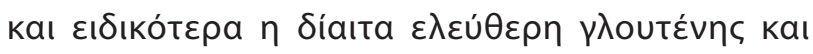

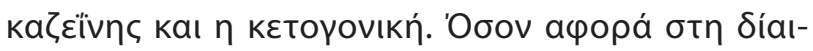

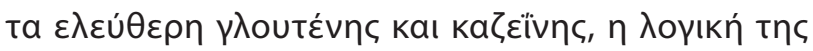

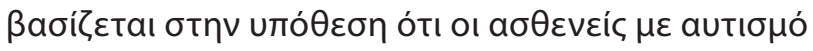

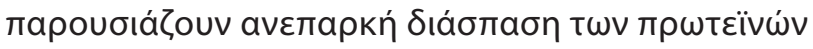

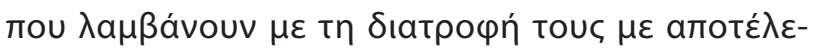

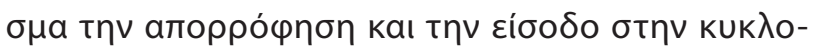

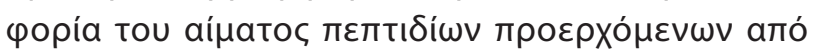

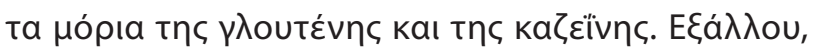

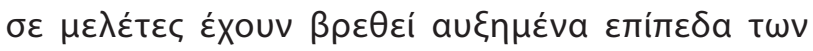

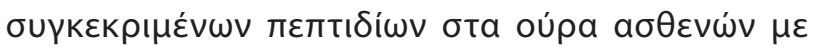

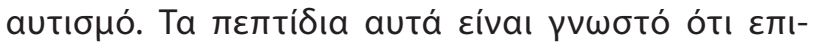

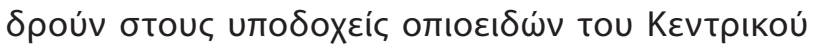

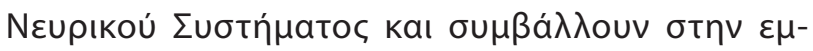

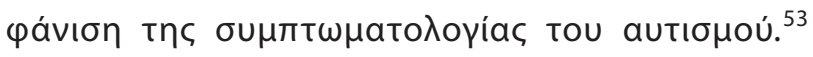

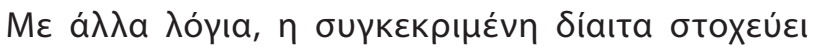

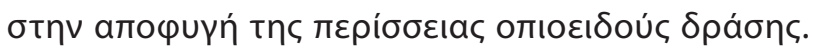

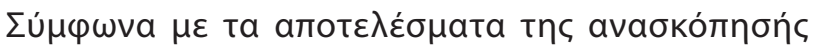




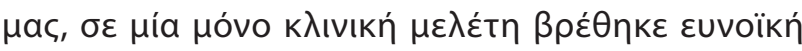

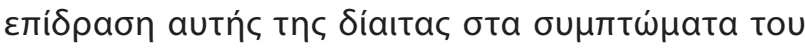

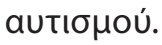

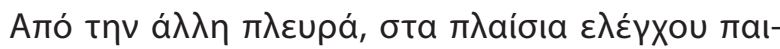

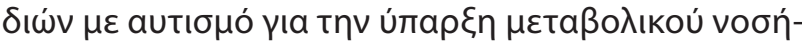

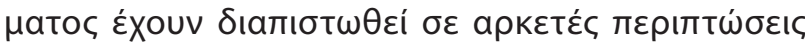

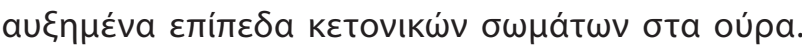

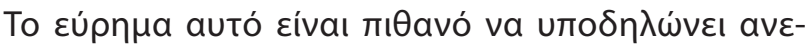

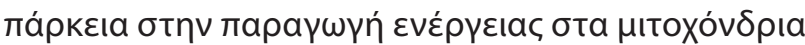

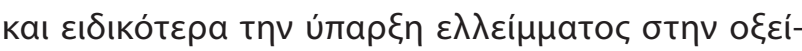

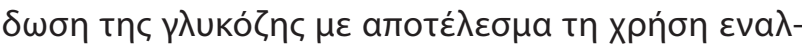

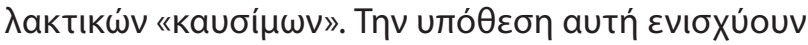

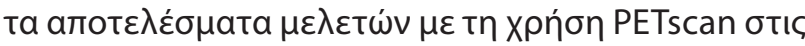

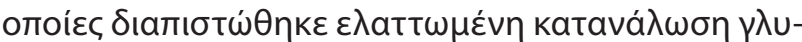

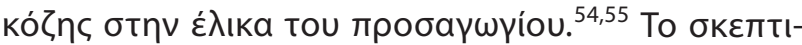

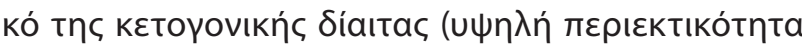

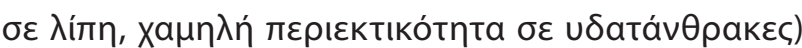

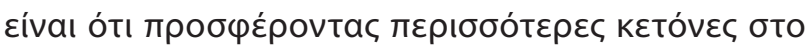

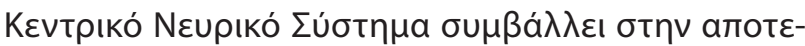

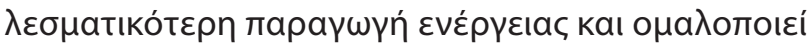

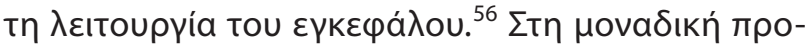

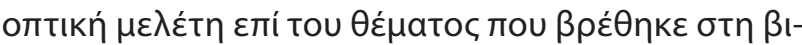

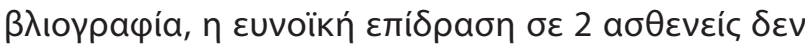

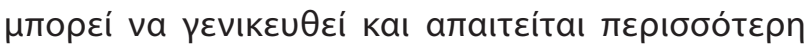

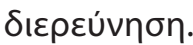

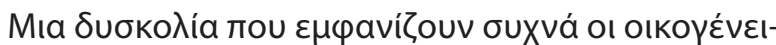

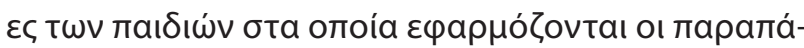

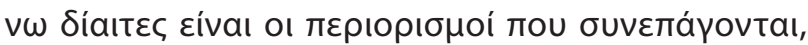

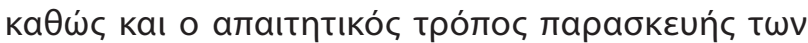

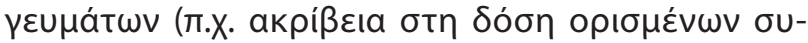

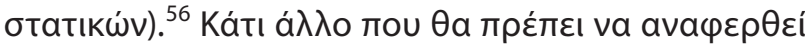

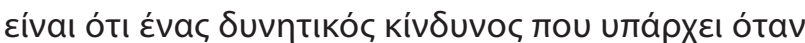

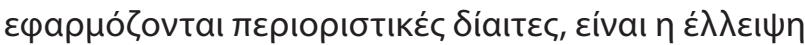

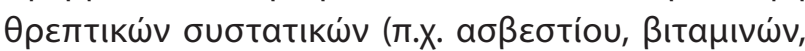

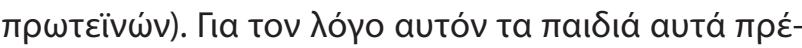

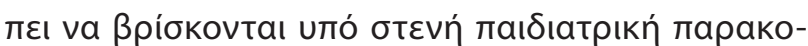

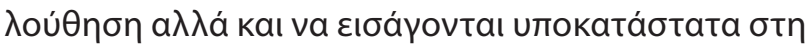

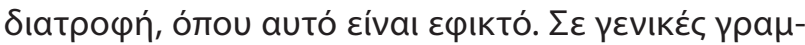

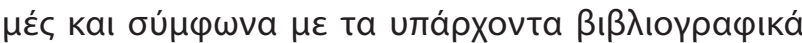

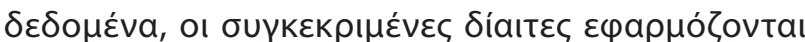

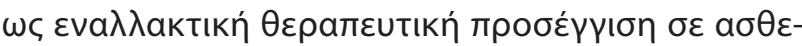

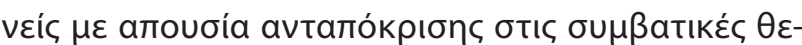

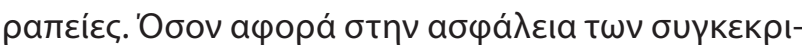

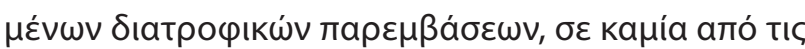

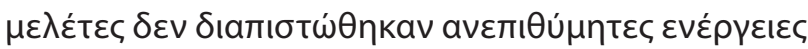

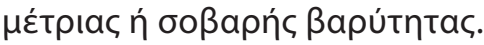

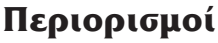

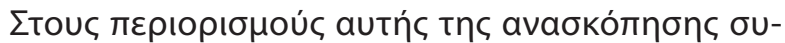

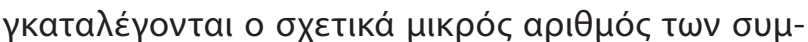

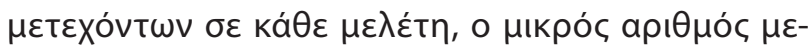

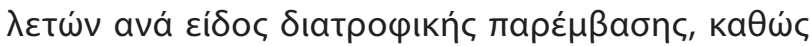

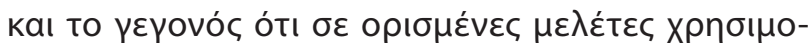

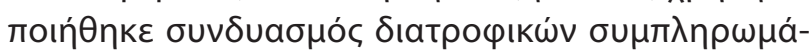

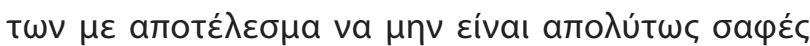

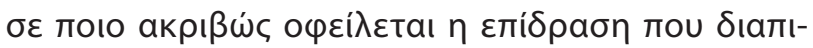

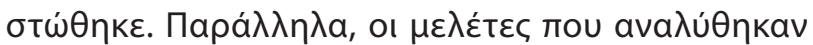

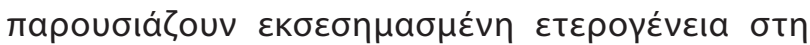

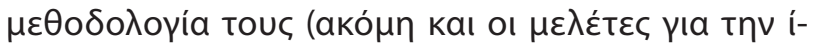

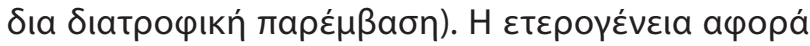

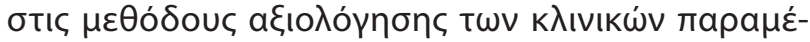

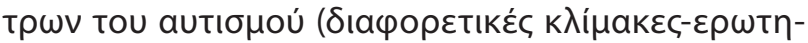

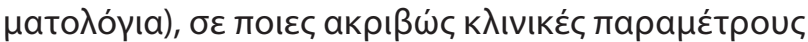

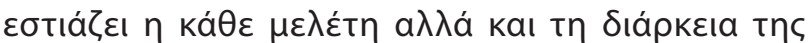

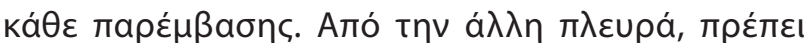

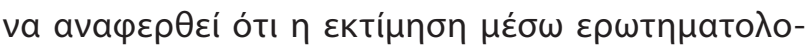

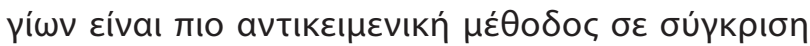

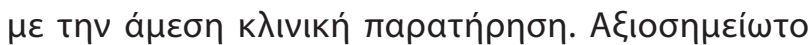

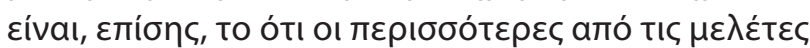

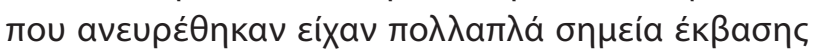

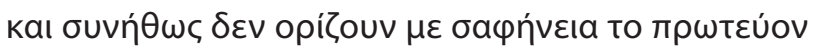

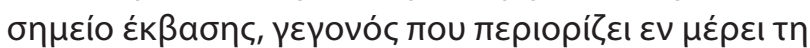

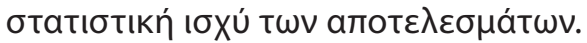

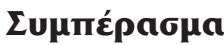

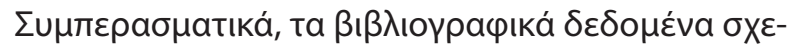

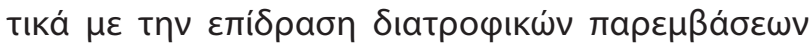

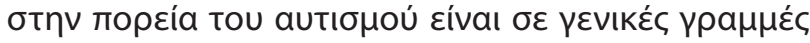

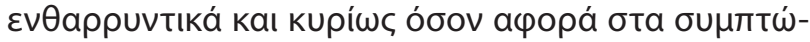

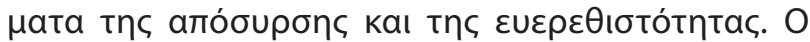

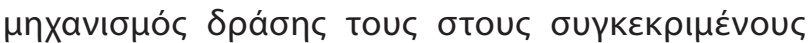

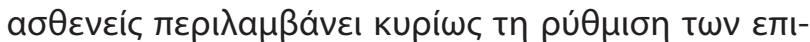

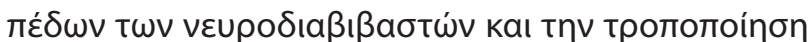

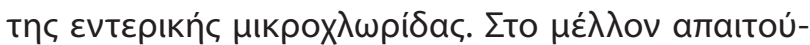

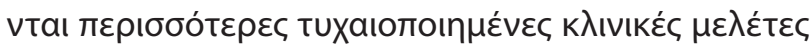

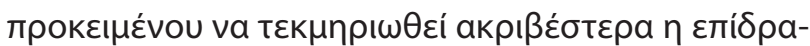

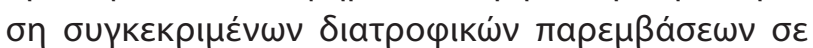

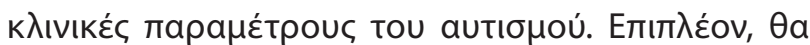

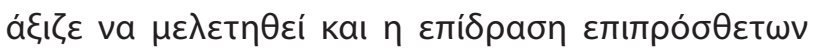

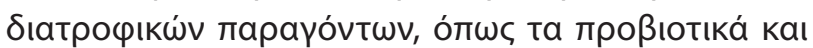

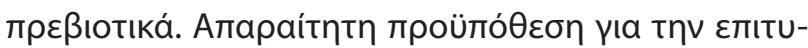

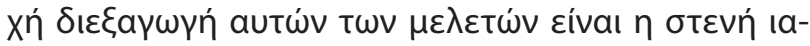

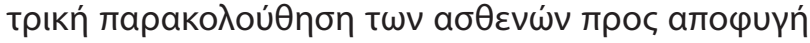

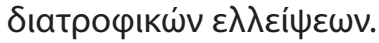




\title{
Is there place for nutrition in the treatment of children with autism spectrum disorder?
}

\author{
M. Gogou, ${ }^{1}$ G. Kolios ${ }^{2}$ \\ '2nd Department of Pediatrics, University General Hospital AHEPA, Thessaloniki, \\ ${ }^{2}$ Laboratory of Pharmacology, Medical School of Democritus University of Thrace, Alexandroupolis, Greece
}

Psychiatriki 2020, 31:57-69

\begin{abstract}
Autism is a neurodevelopmental disorder associated with significant social and financial burden. In recent years there has been an increasing interest in the use of dietary interventions as a complementary therapeutic option for these patients. The aim of this systematic review is to provide literature data about the effect of specific dietary interventions on clinical aspects of children with autism. For this reason, a literature search was conducted using Pubmed as the medical database source. No year-of-publication restriction was placed. Prospective studies conducted in pediatric populations and evaluating changes in clinical aspects of autism were considered. Types of dietary interventions evaluated in these studies included amino acids, fatty acids, vitamins/minerals, as well as specific diets (free of gluten/casein, ketogenic). The underlying mechanism of action of nutritional interventions in this pediatric population mainly includes regulation of neurotransmitters levels, as well as modification of gut microbiota. More specifically, $\mathrm{N}$-acetylcysteine was shown to exert a beneficial effect on symptoms of irritability. This beneficial effect could be attributed to its antiglutamergic and antioxidative properties. With regards to fatty acids, it is known that they are involved in dopamine and serotonin metabolism, while low values of fatty acids have been reported in serum of patients with various neuropsychiatric disorders. However, their administration in children with autism did not make any difference in terms of clinical aspects of the disease. On the other hand, available literature data about effect of $D$-cycloserine, dimethylglycine and vitamins/minerals was either few or controversial. In parallel, we were able to identify in literature clinical studies showing a beneficial effect of gluten/casein-free and ketogenic diet on clinical phenotype of autism. Finally, it should be highlighted that no moderate or serious adverse events were reported in any of the above nutritional interventions. In general, current literature data is encouraging. Nevertheless, more randomized clinical trials are needed to more clearly confirm the effect of specific dietary interventions on clinical aspects of autism.
\end{abstract}

Key words: Autism, amino acids, fatty acids, vitamins, therapeutic diets.

\section{Bıßntoypacpía}

1. Myers SM, Johnson CP; American Academy of Pediatrics Council on Children With Disabilities. Management of children with autism spectrum disorders. Pediatrics 2007, 120:11621182, doi: 10.1542/peds.2007-2362

2. Global Burden of Disease Study 2013 Collaborators. Global, regional, and national incidence, prevalence, and years lived with disability for 301 acute and chronic diseases and injuries in 188 countries, 1990-2013: a systematic analysis for the Global Burden of Disease Study 2013. Lancet 2015, 386:743-800, doi: $10.1016 /$ S0140-6736(15)60692-4
3. Center for Disease Control and Protection. Data \& Statistics on Autism Spectrum Disorder. Available from http://www.cdc.gov/ ncbddd/autism/index.html

4. Lightdale JR. The Gut Speaks: Reframing the Role of Pediatric Gastroenterologists Caring for Children With Autism and Gastrointestinal Symptoms. J Pediatr Gastroenterol Nutr 2016,63:313-314, doi: 10.1097/MPG.0000000000001319

5. Finegold SM, Molitoris D, Song Y, Liu C, Vaisanen ML, Bolte $E$ et al. Gastrointestinal microflora studies in late-onset autism. Clin Infect Dis 2002, 35:6-16, doi: 10.1086/341914 
6. Adams JB, Johansen LJ, Powell LD, Quig D, Rubin RA. Gastrointestinal flora and gastrointestinal status in children with autism--comparisons to typical children and correlation with autism severity. BMC Gastroenterol 2011, 11:22, doi: 10.1186/1471-230X-11-22

7. Francis K. Autism interventions: a critical update. Dev Med Child Neurol 2005, 47:493-499, doi: 10.1017/s0012162205000952

8. Grossi E, Melli S, Dunca D, Terruzzi V. Unexpected improvement in core autism spectrum disorder symptoms after longterm treatment with probiotics. SAGE Open Med Case Rep 2016, 4:2050313X16666231, doi: 10.1177/2050313X16666231

9. Neurology. The official journal of the American Academy of Neurology. Available from http://www.neurology.org/site/misc/ NeurologyFiller.pdf

10. Wink LK, Adams R, Wang Z, Klaunig JE, Plawecki MH, Posey DJ et al. A randomized placebo-controlled pilot study of $\mathrm{N}$-acetylcysteine in youth with autism spectrum disorder. $\mathrm{Mol}$ Autism 2016, 7:26, doi: 10.1186/s13229-016-0088-6

11. Minshawi NF, Wink LK, Shaffer R, Plawecki MH, Posey DJ, Liu H et al. A randomized, placebo-controlled trial of $\mathrm{D}$-cycloserine for the enhancement of social skills training in autism spectrum disorders. Mol Autism 2016, 7:2, doi: 10.1186/s13229-015-0062-8

12. Nikoo M, Radnia H, Farokhnia M, Mohammadi MR, Akhondzadeh S.N-acetylcysteine as an Adjunctive Therapy to Risperidone for Treatment of Irritability in Autism: A Randomized, DoubleBlind, Placebo-Controlled Clinical Trial of Efficacy and Safety. Clin Neuropharmacol 2015, 38:11-17, doi: 10.1097/ WNF.0000000000000063

13. Ghanizadeh A, Moghimi-Sarani E. A randomized double blind placebo controlled clinical trial of $\mathrm{N}$-acetylcysteine added to risperidone for treating autistic disorders. BMC Psychiatry 2013, 13:196, doi: 10.1186/1471-244X-13-196

14. Hardan AY, Fung LK, Libove RA, Obukhanych TV, Nair S, Herzenberg LA et al. A Randomized Controlled Pilot Trial of Oral N-acetylcysteine in Children with Autism. Biol Psychiatry 2012, 71:956-961, doi: 10.1016/j.biopsych.2012.01.014

15. Posey DJ, Kem DL, Swiezy NB, Sweeten TL, Wiegand RE, McDougle CJ. A Pilot Study of D-Cycloserine in Subjects With Autistic Disorder. Am J Psychiatry 2004, 161:2115-2117, doi: 10.1176/appi.ajp.161.11.2115

16. Kern JK, Miller VS, Cauller PL, Kendall PR, Mehta PJ, Dodd M Effectiveness of N,N-Dimethylglycine in Autism and Pervasive Developmental Disorder. J Child Neurol 2001, 16:169-173, doi: 10.1177/088307380101600303

17. Ooi YP, Weng SJ, Jang LY, Low L, Seah J, Teo S et al. Omega-3 fatty acids in the management of autism spectrum disorders: findings from an open-label pilot study in Singapore. Eur J Clin Nutr 2015, 69:969-971, doi: 10.1038/ejcn.2015.28

18. Bent S, Hendren RL, Zandi T, Law K, Choi JE, Widjaja F et al. Internet-Based, Randomized Controlled Trial of Omega-3 Fatty Acids for Hyperactivity in Autism. J Am Acad Child Adolesc Psychiatry 2014, 53:658-666, doi: 10.1016/j.jaac.2014.01.018

19. Voigt RG, Mellon MW, Katusic SK, Weaver AL, Matern D, Mellon $B$ et al. Dietary Docosahexaenoic Acid Supplementation in Children With Autism. J Pediatr Gastroenterol Nutr 2014, 58:715722, doi: 10.1097/MPG.0000000000000260
20. Bent S, Bertoglio K, Ashwood P, Bostrom A, Hendren RL. A Pilot Randomized Controlled Trial of Omega-3 Fatty Acids for Autism Spectrum Disorder. J Autism Dev Disord 2011, 41:545-554, doi: 10.1007/s10803-010-1078-8

21. Amminger GP, Berger GE, Schäfer MR, Klier C, Friedrich MH, Feucht M. Omega-3 Fatty Acids Supplementation in Children with Autism: A Double-blind Randomized, Placebo-controlled Pilot Study. Biol Psychiatry 2007, 61:551-553, doi: 10.1016/j. biopsych.2006.05.007

22. Bertoglio K, Jill James S, Deprey L, Brule N, Hendren RL. Pilot study of the effect of methyl B12 treatment on behavioral and biomarker measures in children with autism. $J$ Altern Complement Med 2010, 16:555-560, doi: 10.1089/acm.2009. 0177

23. Findling RL, Maxwell K, Scotese-Wojtila L, Huang J, Yamashita T, Wiznitzer M. High-Dose Pyridoxine and Magnesium Administration in Children with Autistic Disorder: An Absence of Salutary Effects in a Double-Blind, PlaceboControlled Study. J Autism Dev Disord 1997, 27:467-478, doi: 10.1023/a:1025861522935

24. Levine J, Aviram A, Holan A, Ring A, Barak Y, Belmaker RH. Inositol treatment of autism. J Neural Transm (Vienna) 1997, 104:307-310, doi: 10.1007/s007020050200

25. Tolbert L, Haigler T, Waits MM, Dennis T. Brief Report: Lack of Response in an Autistic Population to a Low Dose Clinical Trial of Pyridoxine Plus Magnesium. J Autism Dev Disord 1993, 23:193-199, doi: 10.1007/bf01066428

26. Dolske MC, Spollen J, McKay S, Lancashire E, Tolbert L. A preliminary trial of ascorbic acid as supplemental therapy for autism. Prog Neuropsychopharmacol Biol Psychiatry 1993, 17:765-774, doi: 10.1016/0278-5846(93)90058-z

27. Martineau J, Barthelemy C, Garreau B, Lelord G. Vitamin B6, magnesium, and combined B6-Mg: therapeutic effects in childhood autism. Biol Psychiatry 1985, 20:467-478, doi: 10.1016/0006-3223(85)90019-8

28. Lelord G, Muh JP, Barthelemy C, Martineau J, Garreau B, Callaway E. Effects of Pyridoxine and Magnesium on AutisticSymptoms--Initial Observations. J Autism Dev Disord 1981, 11:219-230, doi: 10.1007/bf01531686

29. Hyman SL, Stewart PA, Foley J, Cain U, Peck R, Morris DD et al. The Gluten-Free/Casein-Free Diet: A Double-Blind Challenge Trial in Children with Autism. J Autism Dev Disord 2016, 46:205220, doi: 10.1007/s10803-015-2564-9

30. Whiteley P, Haracopos D, Knivsberg AM, Reichelt KL, Parlar S, Jacobsen $\mathrm{J}$ et al. The ScanBritrandomised, controlled, singleblind study of a gluten- and casein-free dietary intervention for children with autism spectrum disorders. Nutr Neurosci 2010, 13:87-100, doi: 10.1179/147683010X12611460763922

31. Elder JH, Shankar M, Shuster J, Theriaque D, Burns S, Sherrill L. The gluten-free, casein-free diet in autism: results of a preliminary double blind clinical trial. J Autism Dev Disord 2006, 36:413-420, doi: 10.1007/s10803-006-0079-0

32. Evangeliou A, Vlachonikolis I, Mihailidou H, Spilioti M, Skarpalezou A, Makaronas $\mathrm{N}$ et al. Application of a ketogenic diet in children with autistic behavior: pilot study. J Child Neurol 2003, 18:113-118, doi: 10.1177/08830738030180020501 
33. Knivsberg AM, Reichelt KL, Høien T, Nødland M. A Randomised, controlled study of dietary intervention in autistic syndromes. Nutr Neurosci 2002, 5:251-261, doi: 10.1080/10284150290028945

34. Purcell A, Jeon O, Zimmerman A, Blue ME, Pevsner J. Postmortem brain abnormalities of the glutamate neurotransmitter system in autism. Neurology 2001, 57:1618-1628, doi: 10.1212/wnl.57.9.1618

35. Fatemi SH, Halt AR, Stary JM, Kanodia R, Schulz SC, Realmuto GR. Glutamic acid decarboxylase 65 and $67 \mathrm{kDa}$ proteins are reduced in autistic parietal and cerebellar cortices. Biol Psychiatry 2012, 52:805-810, doi: 10.1016/s00063223(02)01430-0

36. Shinohe A, Hashimoto K, Nakamura K, Tsujii M, Iwata Y, Tsuchiya $\mathrm{KJ}$ et al. Increased serum levels of glutamate in adult patients with autism. Prog Neuropsychopharmacol Biol Psychiatry 2006, 30:1472-1477, doi: 10.1016/j.pnpbp.2006.06.013

37. Modi ME, Young LJ. D-cycloserine facilitates socially reinforced learning in an animal model relevant to autism spectrum disorders. Biol Psychiatry 2011, 70:298-304, doi: 10.1016/j. biopsych.2011.01.026

38. Baker DA, Xi ZX, Shen H, Swanson CJ, Kalivas PW. The origin and neuronal function of in vivo nonsynaptic glutamate. $J$ Neurosci 2002, 22:9134-9141, PMID: 12388621

39. Ghanizadeh A, Akhondzadeh S, Hormozi M, Makarem A, Abotorabi-Zarchi M, Firoozabadi A. Glutathione-related factors and oxidative stress in autism, a review. Curr Med Chem 2012, 19:4000-4005, doi: 10.2174/092986712802002572

40. Damodaran LPM, Arumugam G. Urinary oxidative stress markers in children with autism. Redox Rep 2011, 16:216-222, doi: 10.1179/1351000211Y.0000000012

41. Livine S, Myhre G, Smith G, Bums J. Effect of nutritional supplement containing $\mathrm{N}, \mathrm{N}$-dimethylglycine (DMG) on the racing standard bred. Equine Pract 1982, 4:16-18

42. Wainwright PE. Dietary essential fatty acids and brain function: a developmental perspective on mechanisms. Proc Nutr Soc 2002, 61:61-69, doi:10.1079/pns2001130

43. Bazan NG. Lipid signalling in neural plasticity, brain repair, and neuroprotection. Mol Neurobiol 2005, 32:89-103, doi: 10.1385/ $\mathrm{MN}: 32: 1: 089$

44. Hibbeln JR, Umhau JC, Linnoila M, George DT, Ragan PW, Shoaf SE et al. A replication study of violent and nonviolent subjects: cerebrospinal fluid metabolites of serotonin and dopamine are predicted by plasma essential fatty acids. Biol Psychiatry 1998, 44:243-249, doi: 10.1016/s0006-3223(98)00143-7

45. Bell JG, MacKinlay EE, Dick JR, MacDonald DJ, Boyle RM, Glen AC. Essential fatty acids and phospholipase A2 in autistic spectrum disorders. Prostaglandins Leukot Essent Fatty Acids 2004, 71:201-204, doi: 10.1016/j.plefa.2004.03.008

46. Noaghiul S, Hibbeln JR. Cross-national comparisons of seafood consumption and rates of bipolar disorders. Am J Psychiatry 2003, 160:2222-2227, doi: 10.1176/appi.ajp.160.12.2222

47. Raz R, Gabis L. Essential fatty acids and attention-deficit-hyperactivity disorder: a systematic review. Dev Med Child Neurol 2009, 51:580-592, doi: 10.1111/j.1469-8749.2009.03351.x

48. Adams JB, Audhya T, Vogelaar E. Nutritional abnormalities in autism and the effect of nutritional supplementation. Paper pre- sented at Conference of the National Autism Society of America, Pittsburgh, 16-20 July, 2003

49. Golnik AE, Ireland M. Complementary alternative medicine for children with autism: a physician survey. J Autism Dev Disord 2009, 39:996-1005, doi: 10.1007/s10803-009-0714-7

50. Coleman M, Steinberg G, Tippett J, Bhagavan HN, Coursin $\mathrm{DB}$, Gross $\mathrm{M}$ et al. A preliminary study of the effect of pyridoxine administration in a subgroup of hyperkinetic children: a double-blind crossover comparison with methylphenidate. Biol Psychiatry 1979, 14:741-751, PMID: 497303

51. Tolbert LC, Thomas TN, Middaugh LD, Zemp JW. Ascorbate blocks amphetamine-induced turning behavior in rats with unilateral nigro-striatal lesions. Brain Res Bull 1979, 4:43-48, doi: 10.1016/0361-9230(79)90056-x

52. Rahman S, Neuman RS. Myo-inositol reduces serotonin (5-HT2) receptor induced homologous and heterologous desensitization. Brain Res 1993, 631:349-351, doi: 10.1016/0006-8993 (93) $91557-9$

53. Whiteley P, Rodgers J, Savery D, Shattock P. A Gluten-Free Diet as an Intervention for Autism and Associated Spectrum Disorders: Preliminary Findings. Autism 1999, 3:45-65, doi: $10.1177 / 1362361399003001005$

54. Poggi-Travert $F$, Martin D, Billette de Villemeur T, Bonnefont JP, Vassault A, Rabier D et al. Metabolic intermediates in lactic acidosis: compounds, samples and interpretation. $J$ Inherit Metab Dis 1996, 19:478-488, doi: 10.1007/bf01799109

55. Haznedar MM, Buchsbaum MS, Wei TC, Hof PR, Cartwright C, Bienstock CA et al. Limbic circuitry in patients with autism spectrum disorders studied with positron emission tomography and magnetic resonance imaging. Am J Psychiatry 2000, 157:1994-2001, doi: 10.1176/appi.ajp.157.12.1994

56. Posey DJ, McDougle CJ. Pharmacotherapeutic management of autism. Expert Opin Pharmacother 2001, 2:587-600, doi: 10.1517/14656566.2.4.587

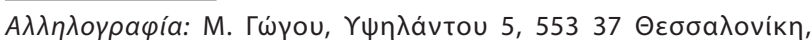

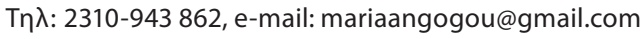




\title{
Eıठı́ó á $\operatorname{\theta \rho o}$ Special article
}

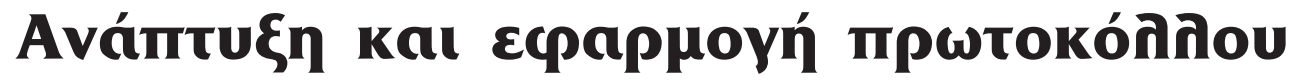

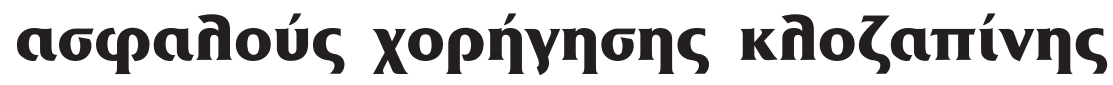

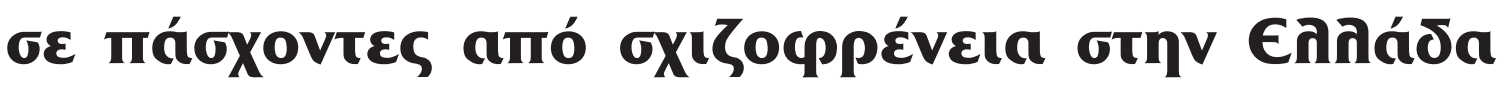

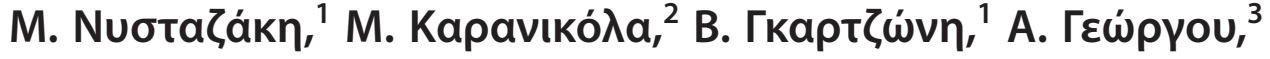

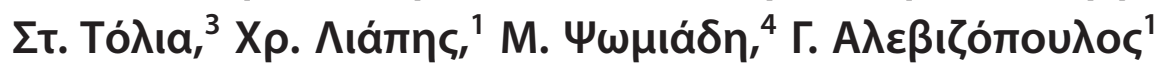

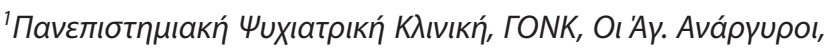

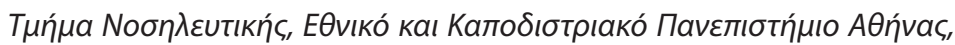

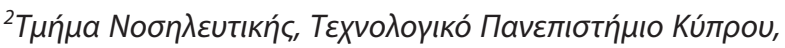

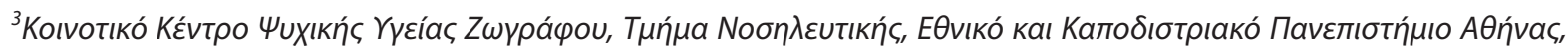

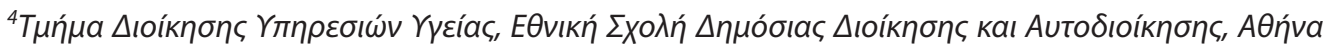

Uuxıатрıкń 2020, 31:70-81

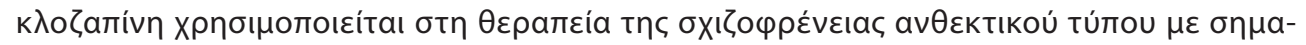

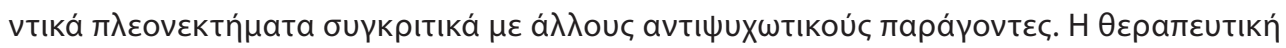

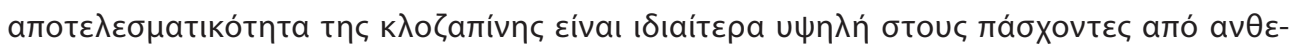

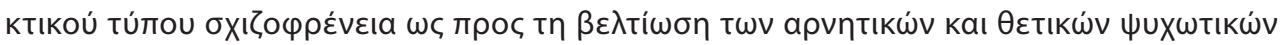

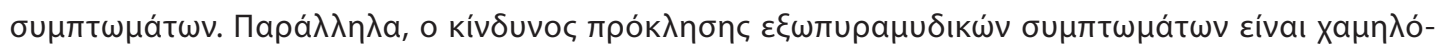

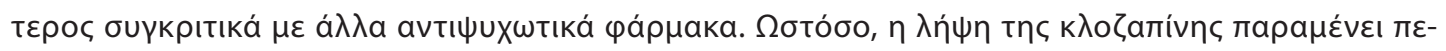

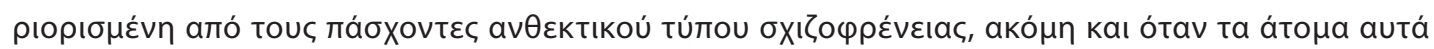

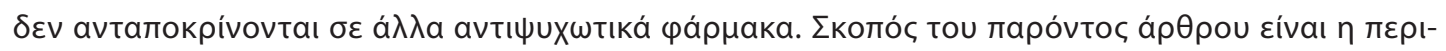

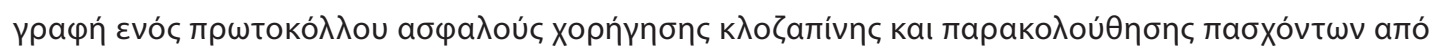

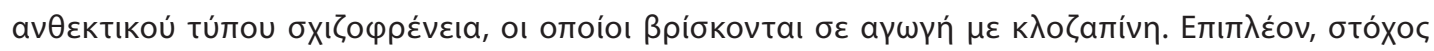

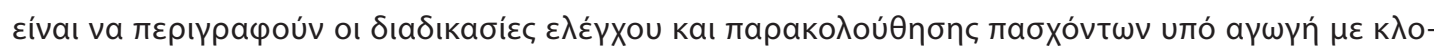

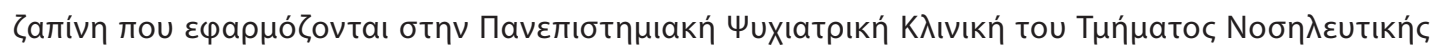

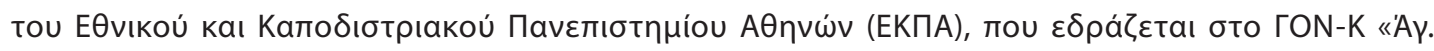

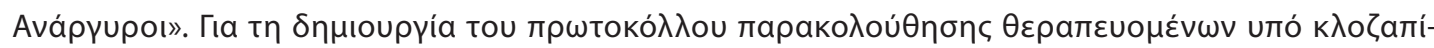

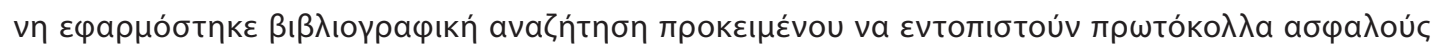

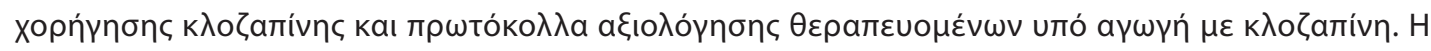




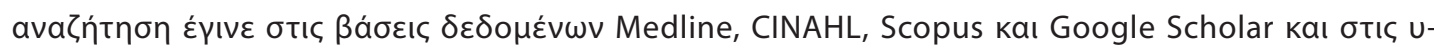

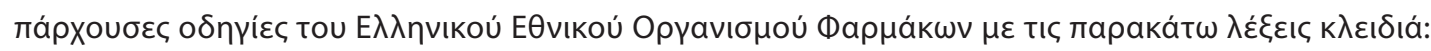

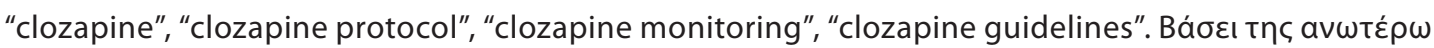

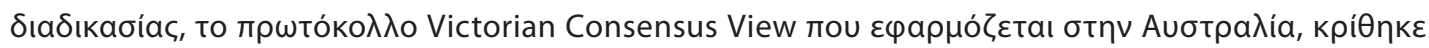

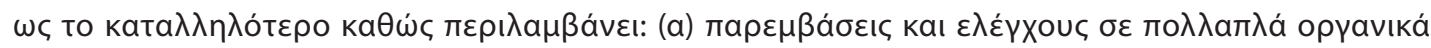

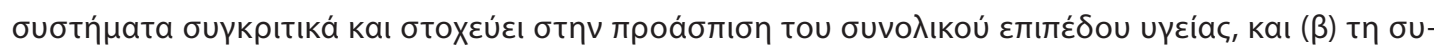

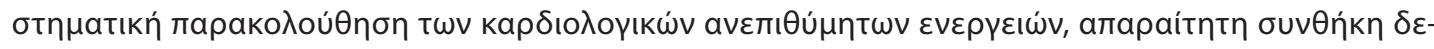

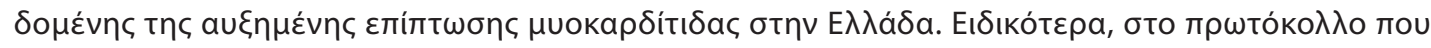

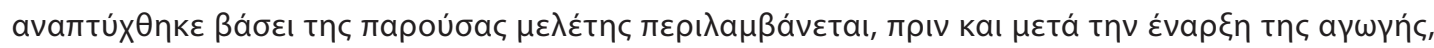

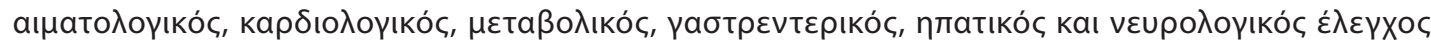

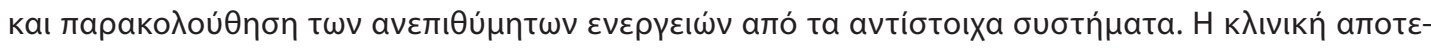

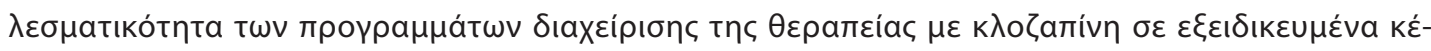

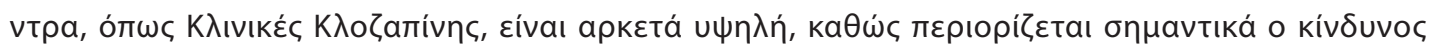

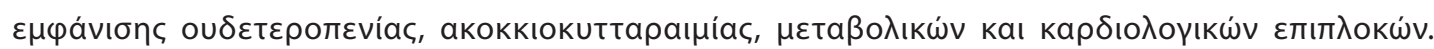

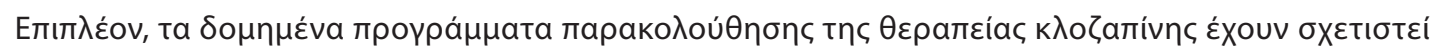

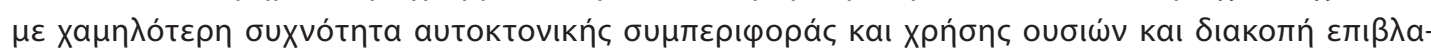

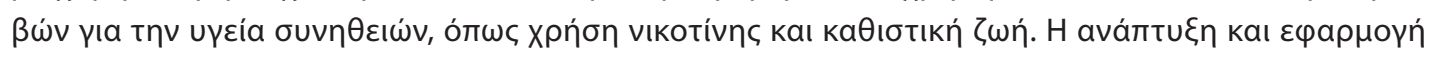

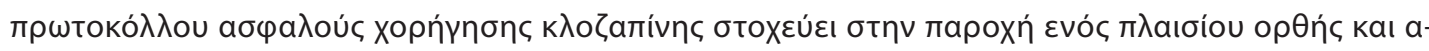

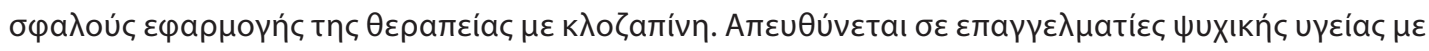

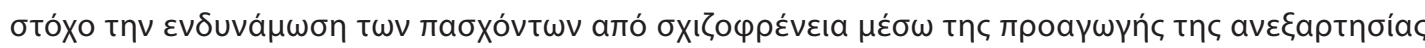

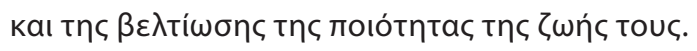

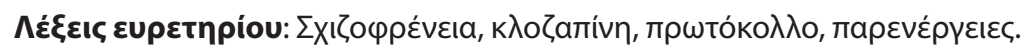

\section{Eıбaywyń}

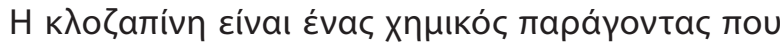

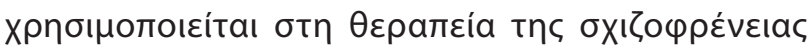

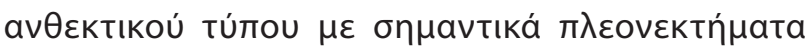

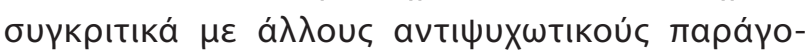

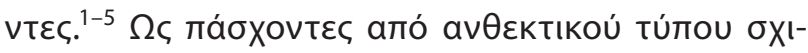

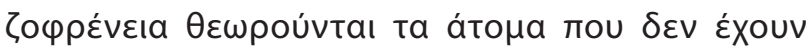

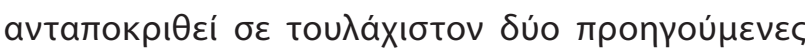

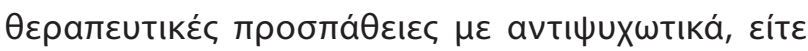

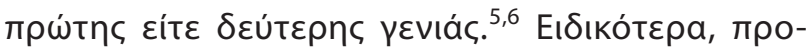

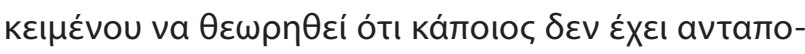

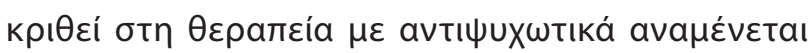

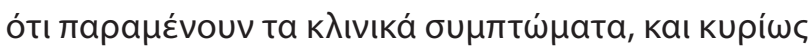

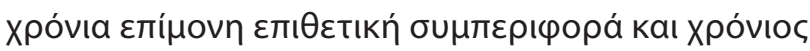

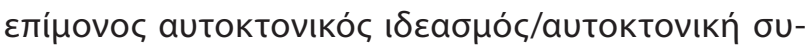

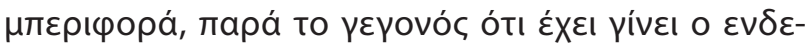

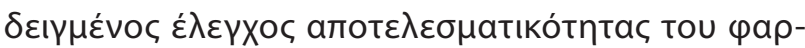

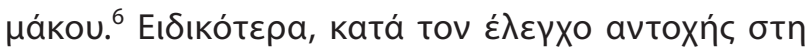

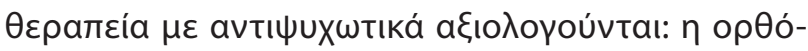

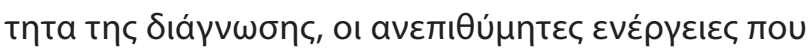

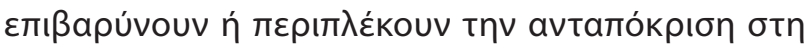

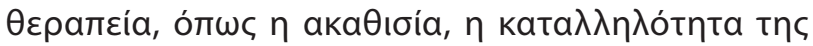

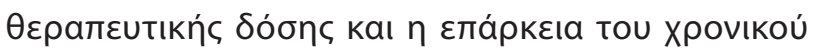

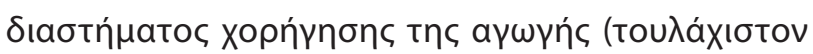

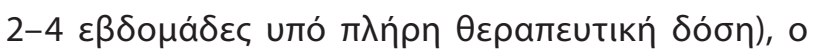

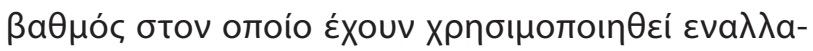

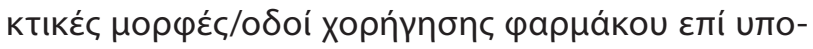

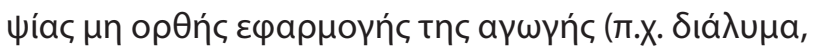

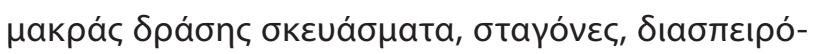

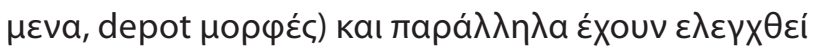

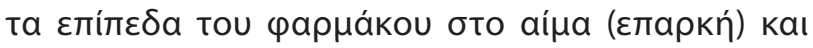

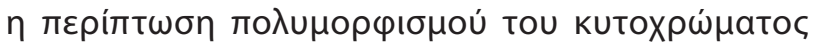

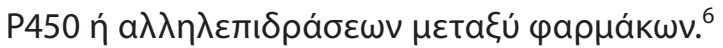

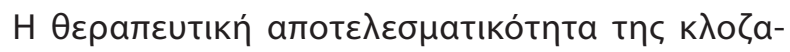

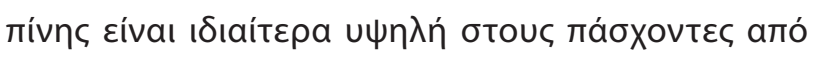

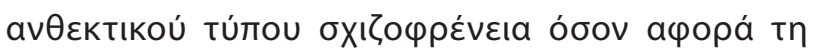

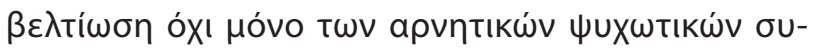

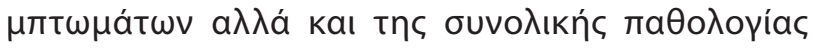

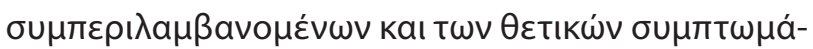

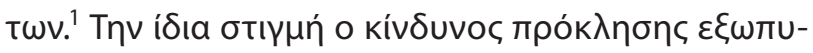

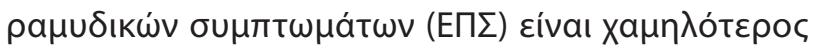

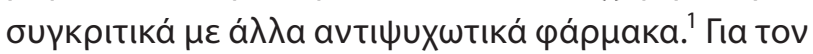




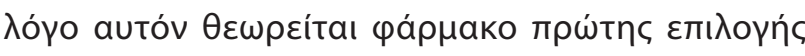

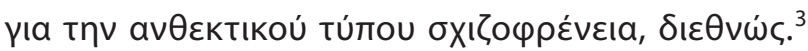

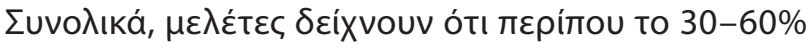

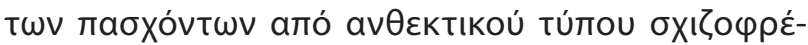

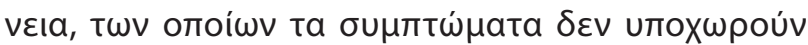

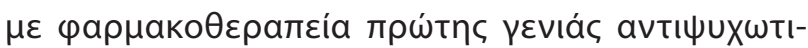

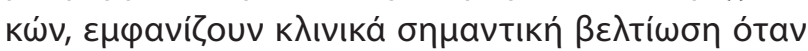

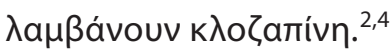

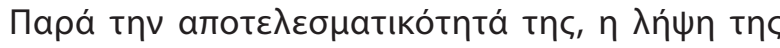

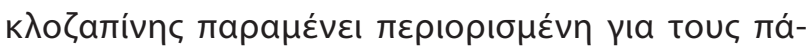

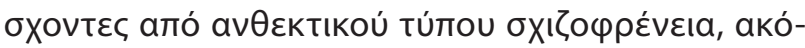

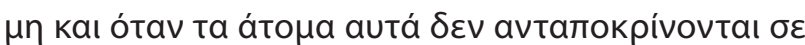

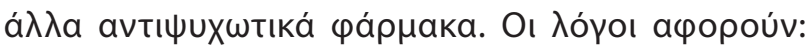

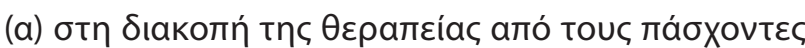

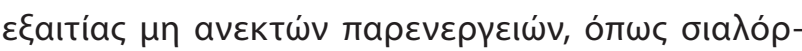

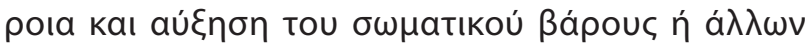

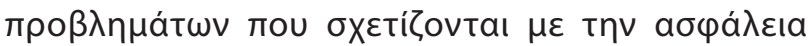

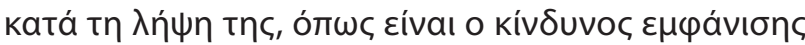

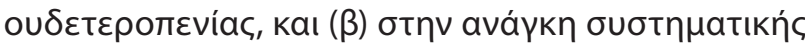

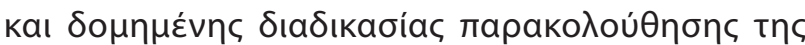

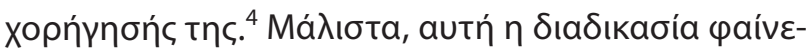

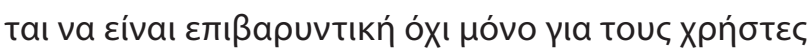

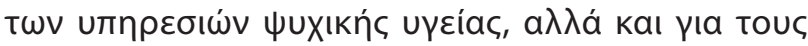

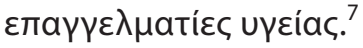

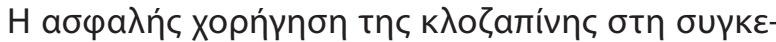

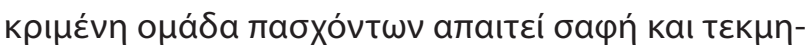

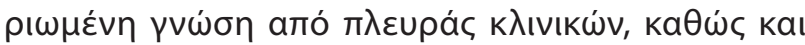

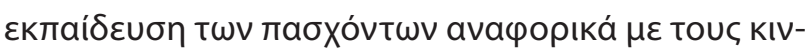

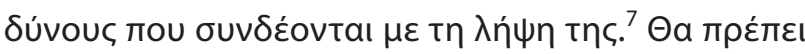

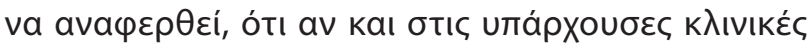

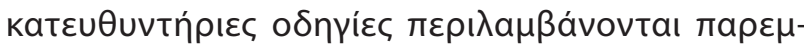

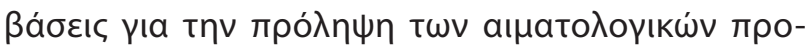

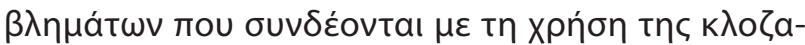

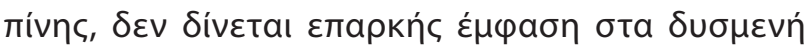

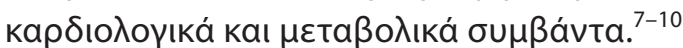

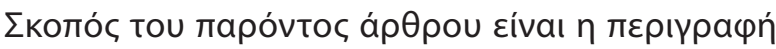

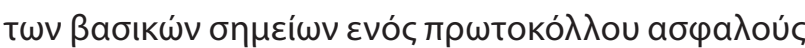

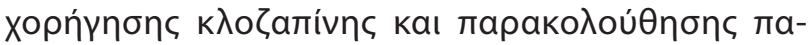

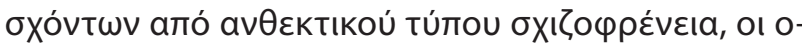

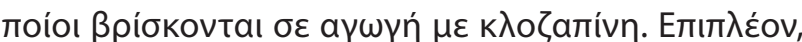

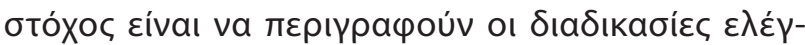

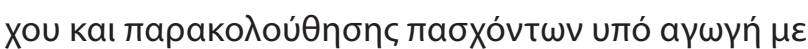

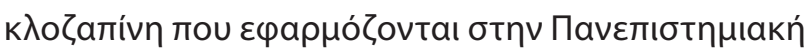

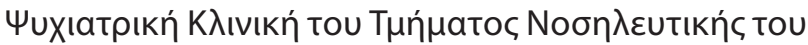

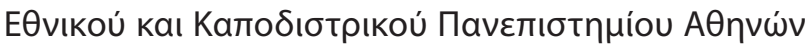

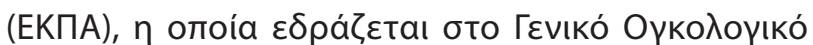

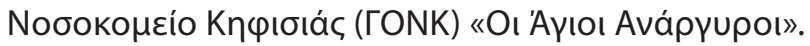

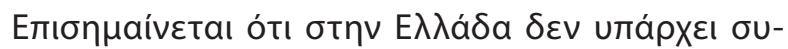

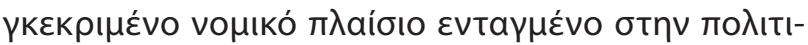

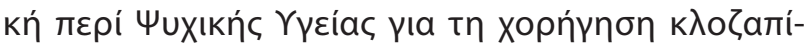

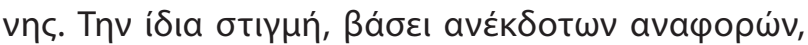

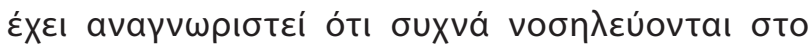

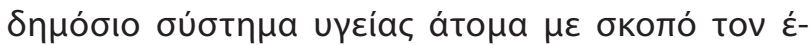

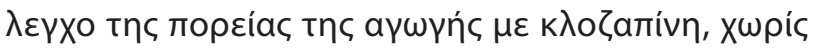

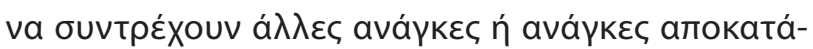

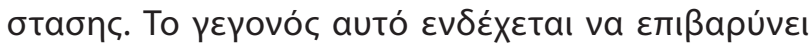

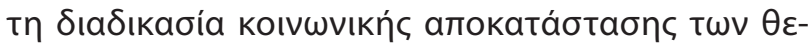

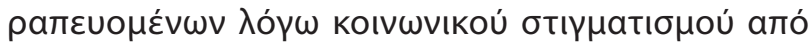

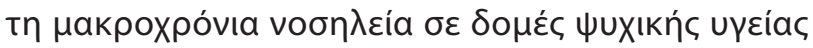

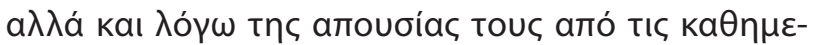

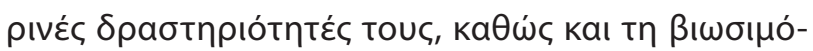

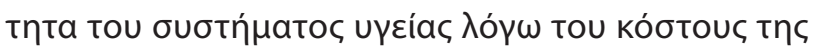

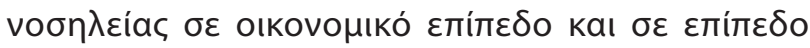

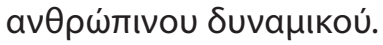

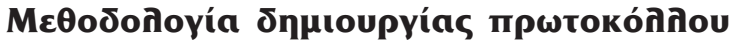

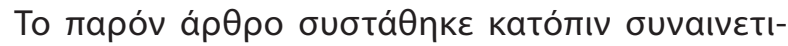

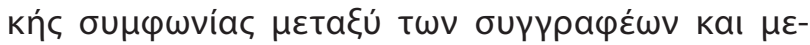

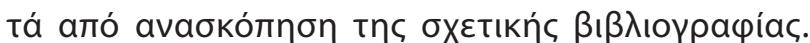

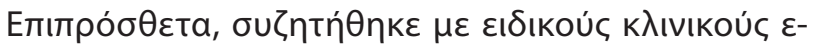

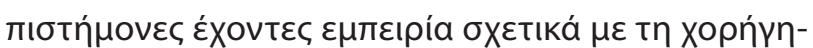

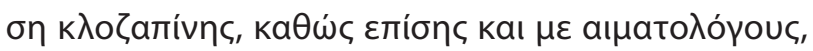

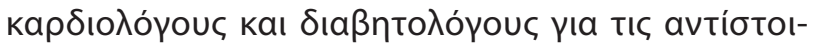

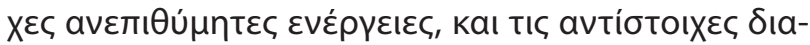

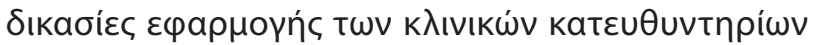

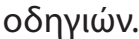

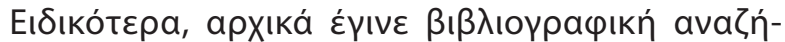

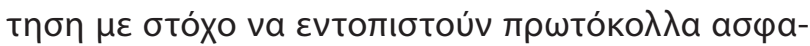

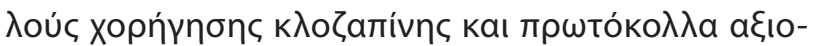

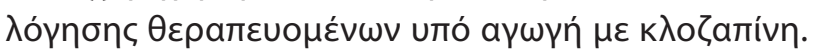

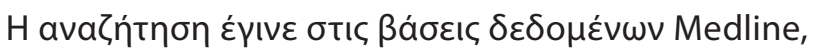
CINAHL, Scopus kaı Google Scholar kaı otıc uாáp-

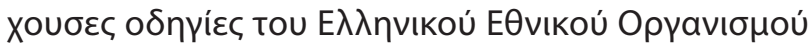

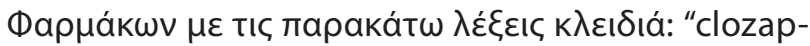
ine", "clozapine protocol", "clozapine monitoring",

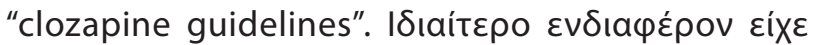

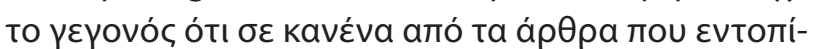

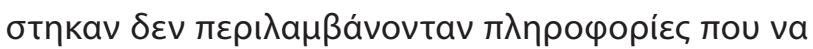

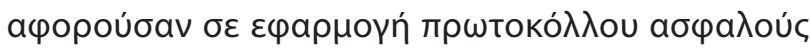

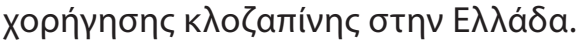




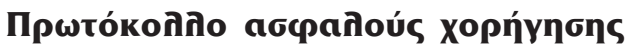 кก̀oろamívns}

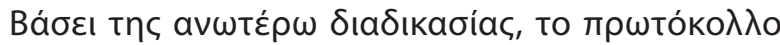

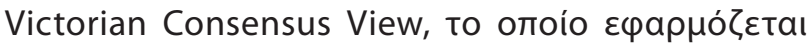

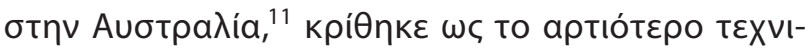

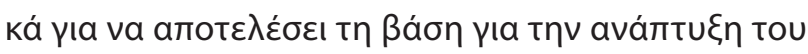

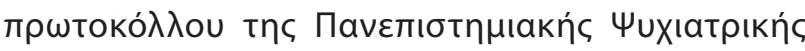

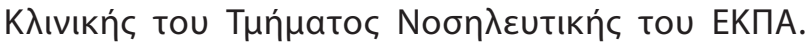

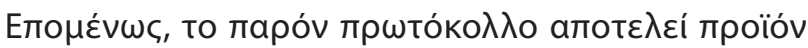

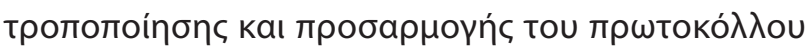
Victorian Consensus View. ${ }^{11}$

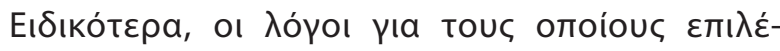

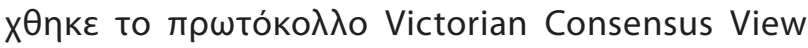

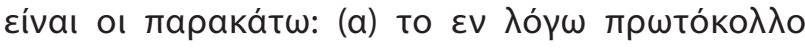

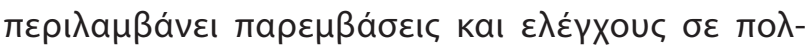

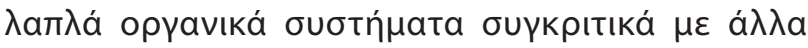

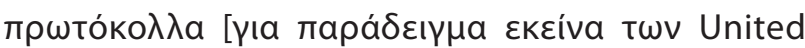
States Food and Drug Administration (USA FDA), ${ }^{12}$ Maudsley Guidelines, ${ }^{13}$ The British Association for

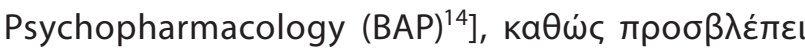

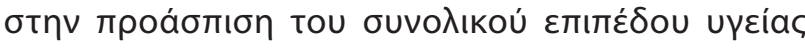

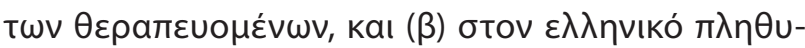

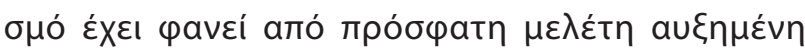

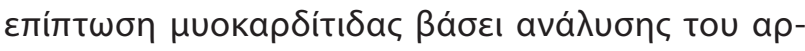

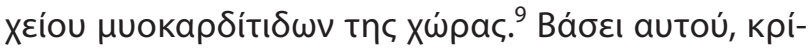

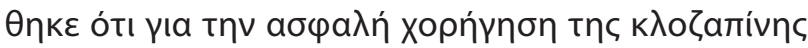

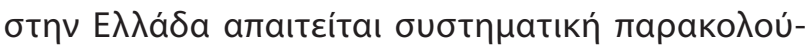

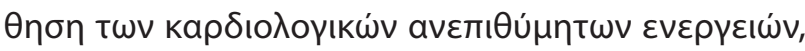

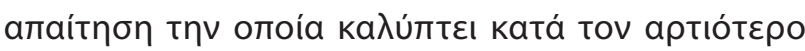
тро́то то пршто́ко $\lambda$ о Victorian Consensus View. ${ }^{11}$

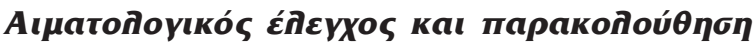

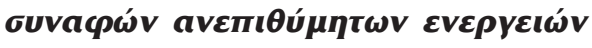

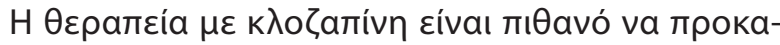

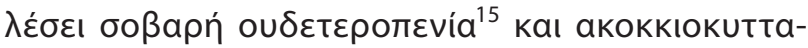

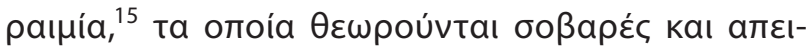

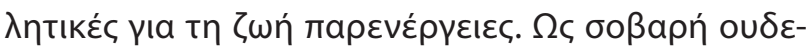

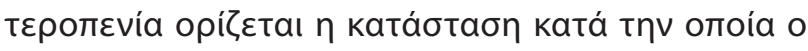

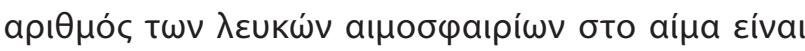

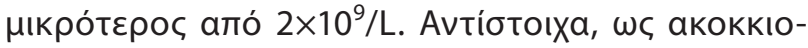

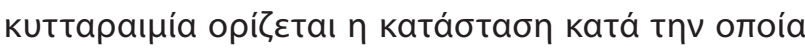

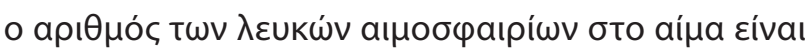

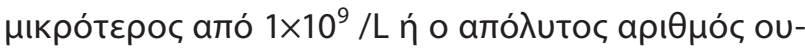

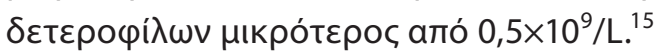

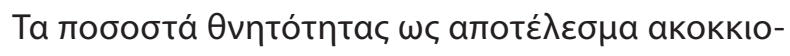

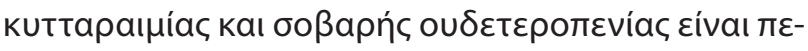

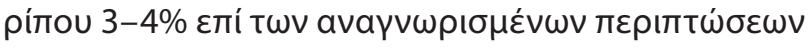

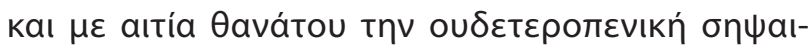

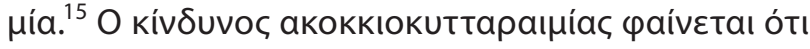

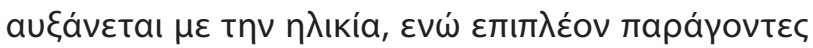

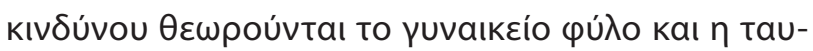

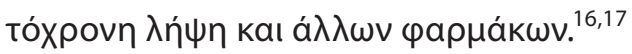

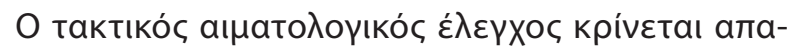

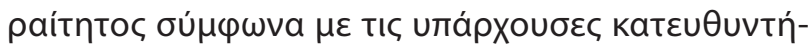

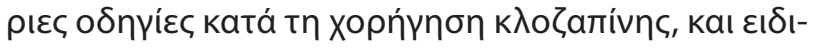

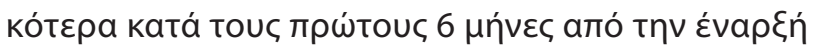

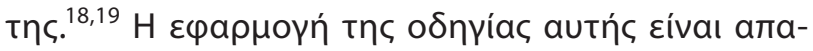

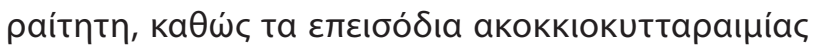

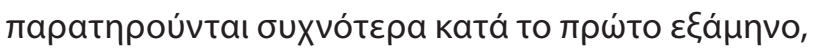

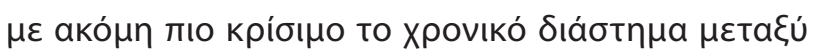

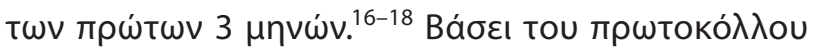

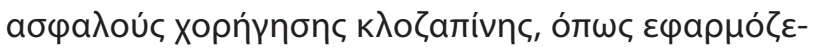

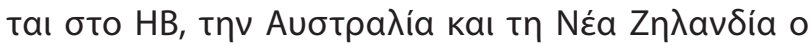

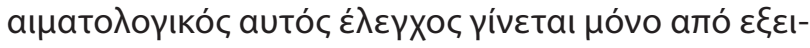

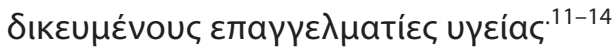

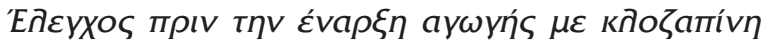

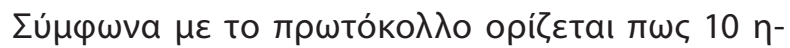

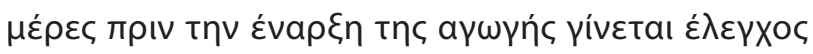

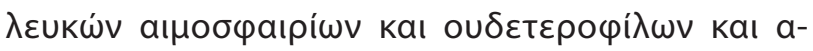

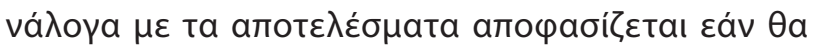

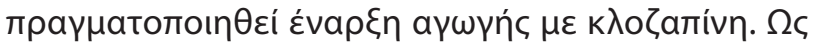

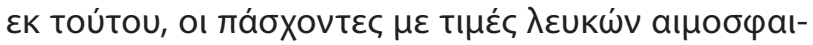

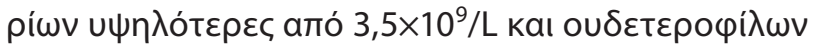

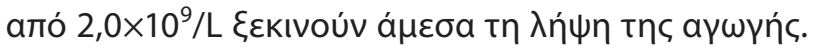

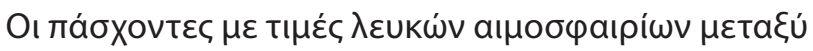

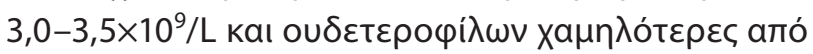

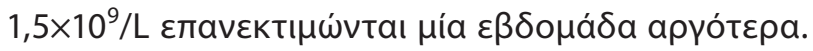

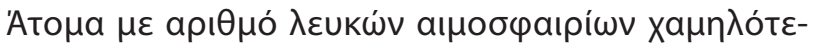

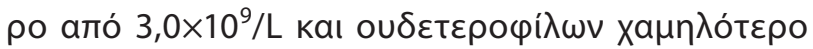

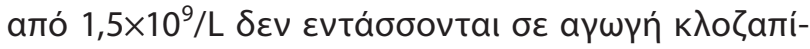
vnc.

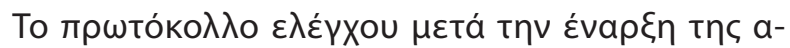

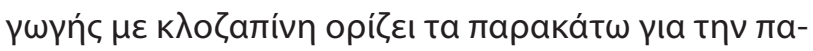

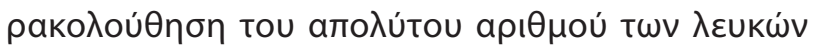

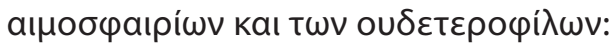

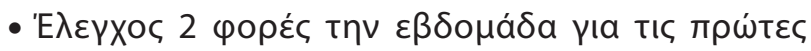

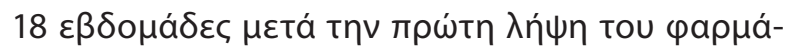
Kou. ${ }^{11,18,19}$ 


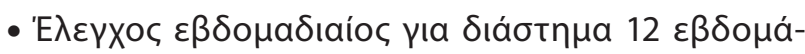

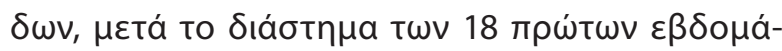
$\delta \omega v$ a

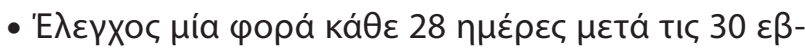

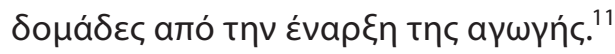

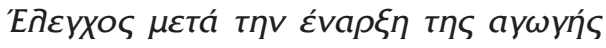

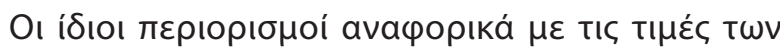

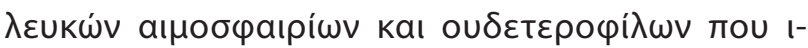

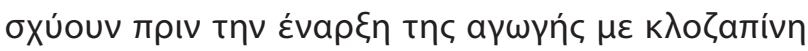

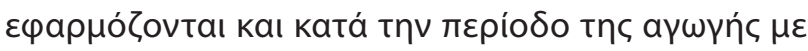

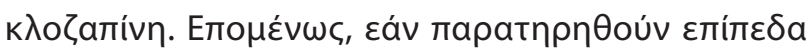

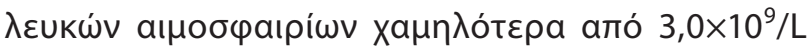

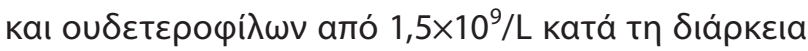

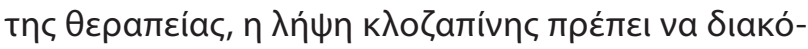

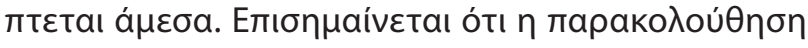

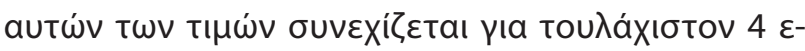

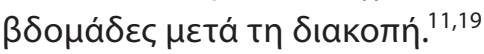

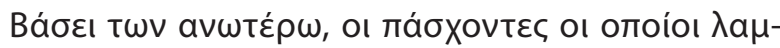

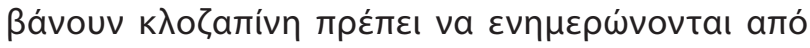

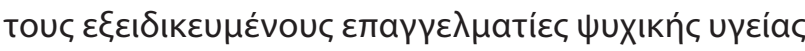

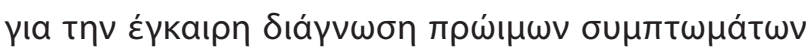

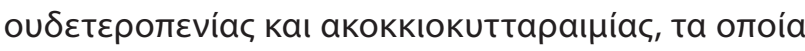

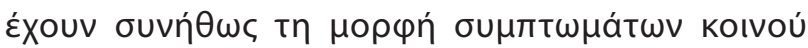

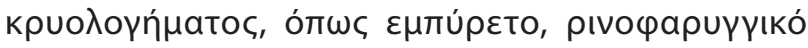

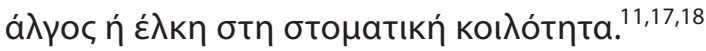

\section{Kapঠıоกоуıкós éneyXos}

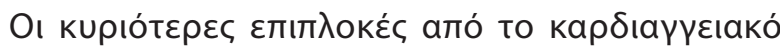

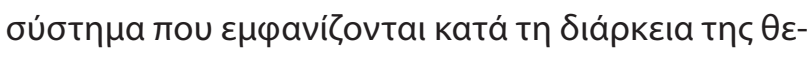

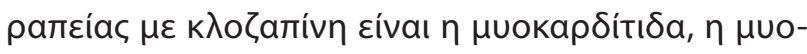

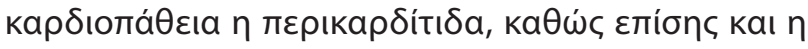

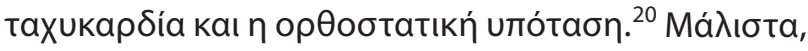
$\lambda$

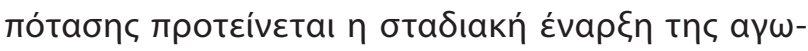

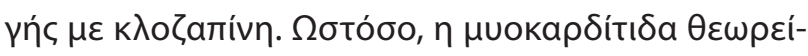

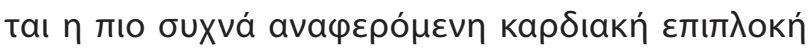

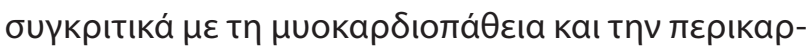

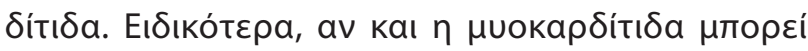

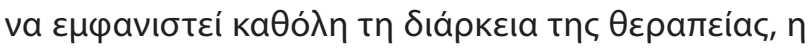

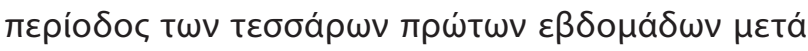

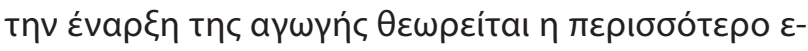

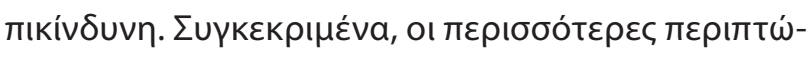

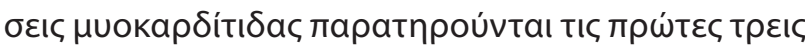

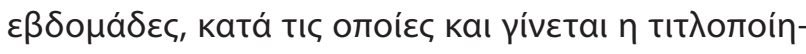

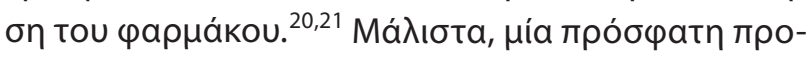

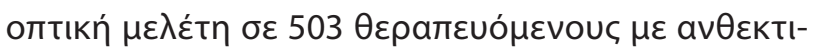

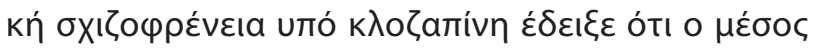

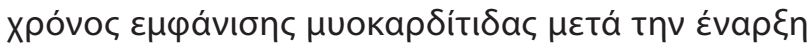

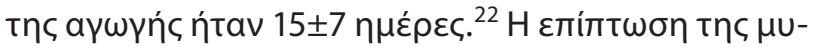

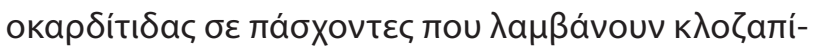

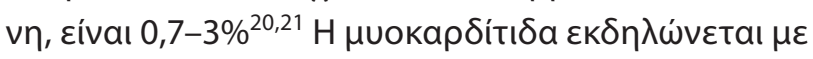

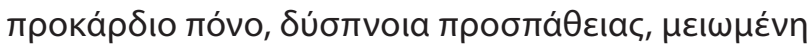

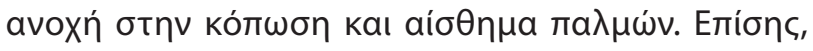

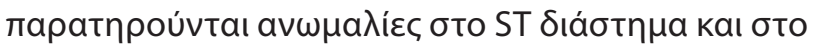

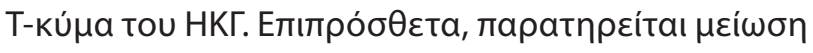

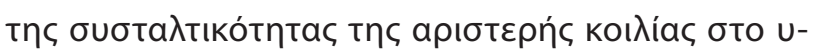

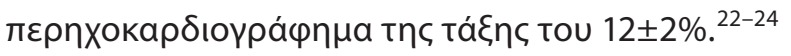

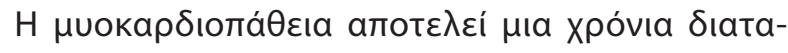

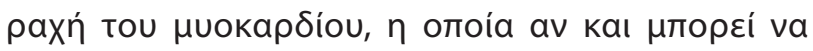

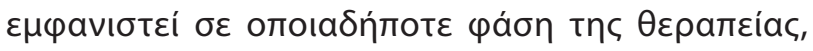

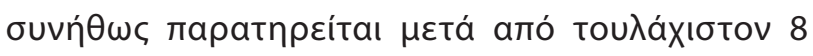

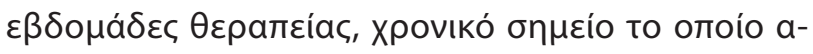

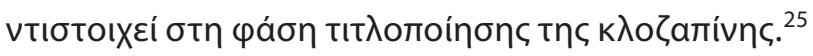

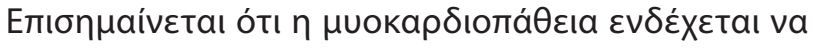

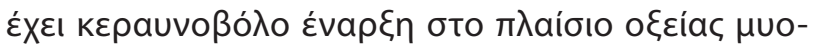

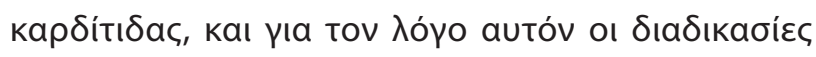

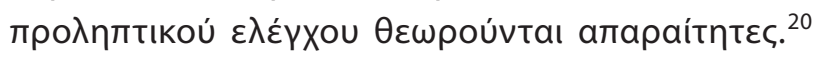

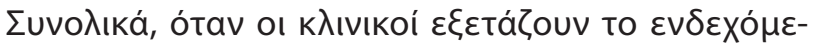

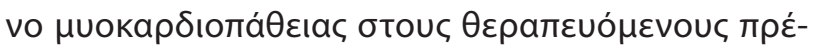

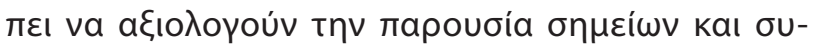

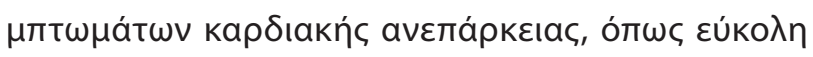

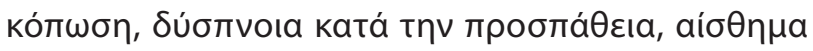

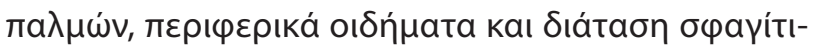
$\delta \omega v .^{11}$

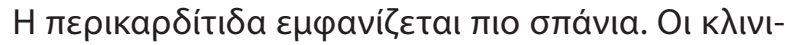

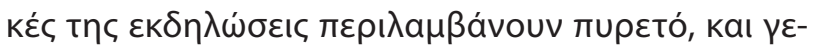

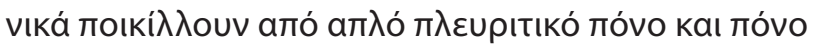

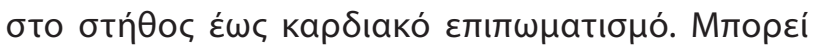

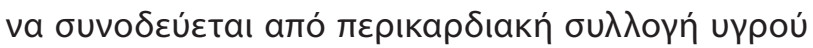

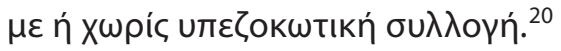

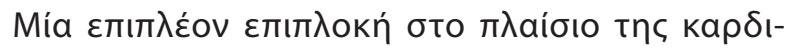

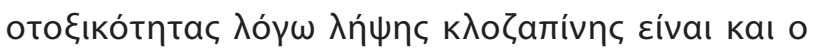

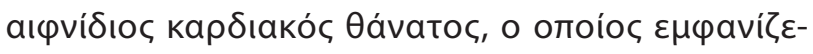

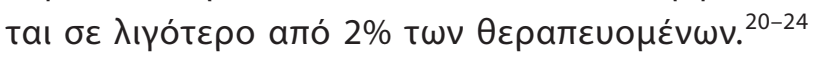

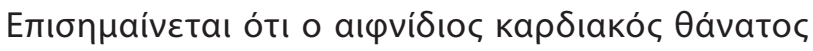

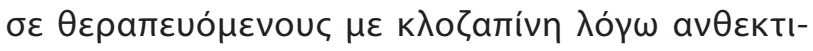

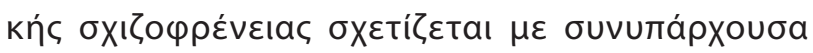

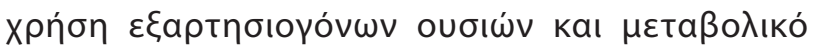

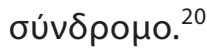




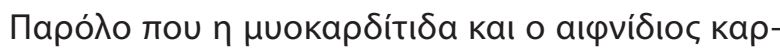

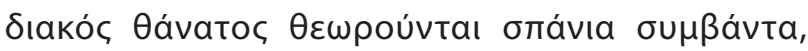

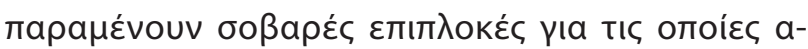

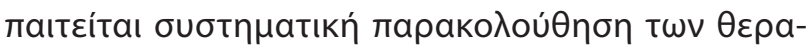

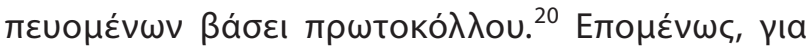

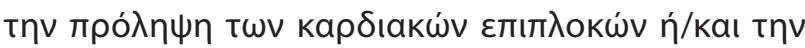

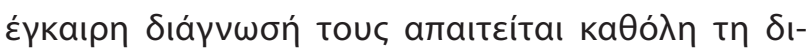

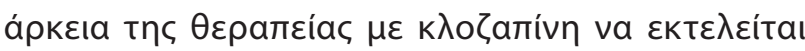

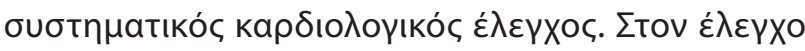

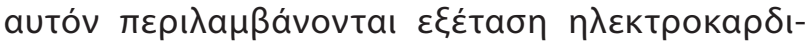

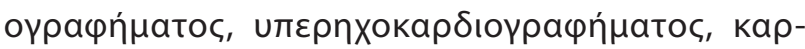

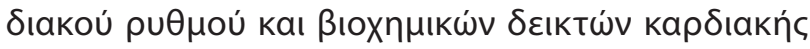
$\lambda \varepsilon$ itoupyíac.. ${ }^{24}$

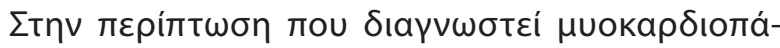

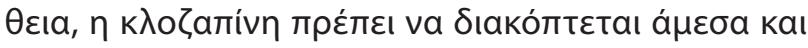

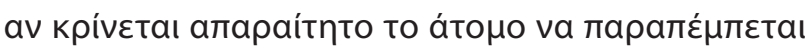

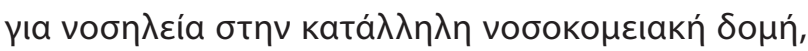

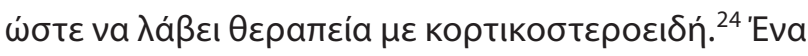

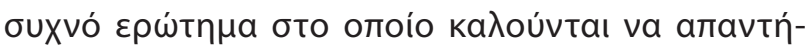

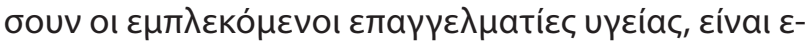

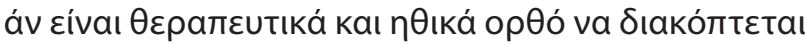

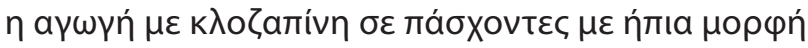

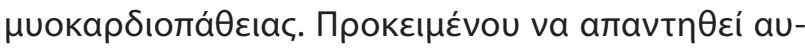

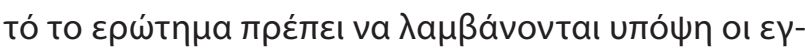

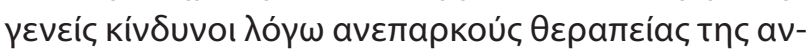

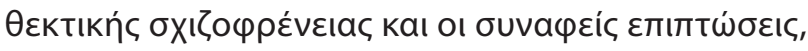

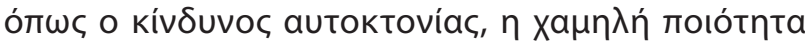

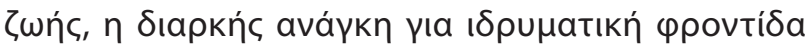

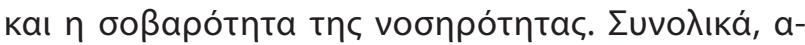

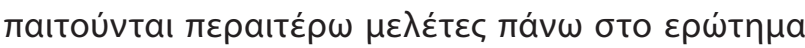
autó,

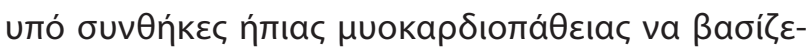

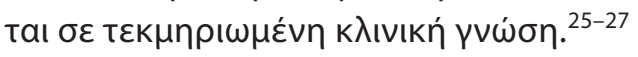

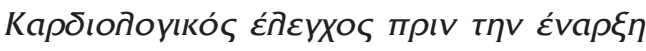

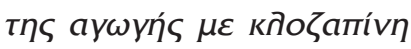

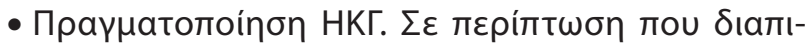

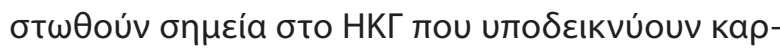

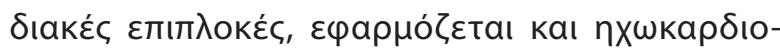

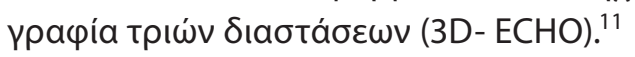

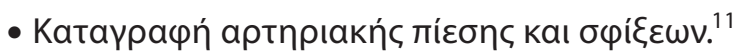

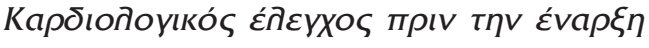

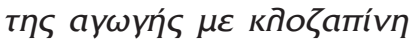

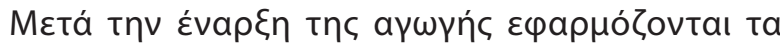
паракátw:

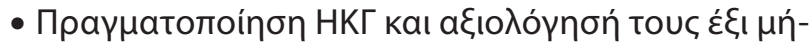

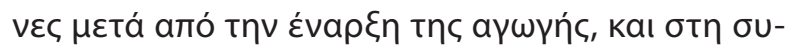

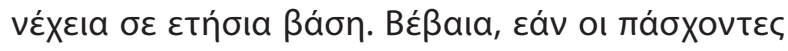

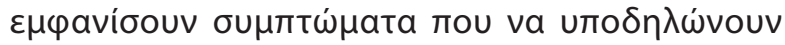

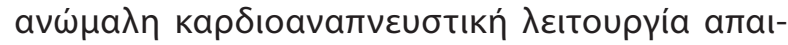

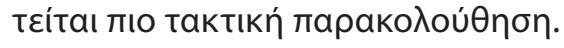

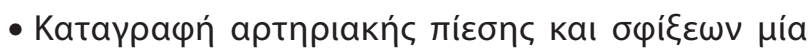

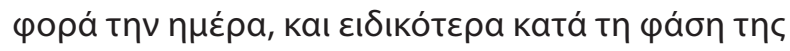

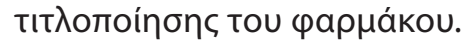

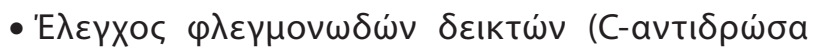

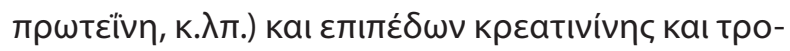

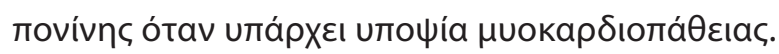

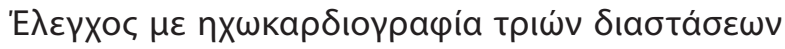

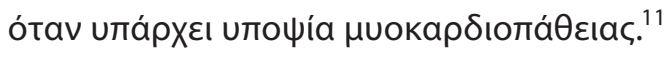

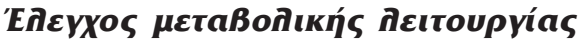

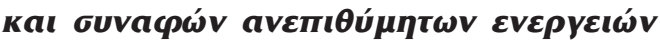

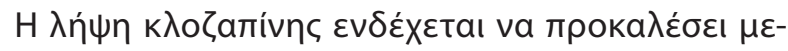

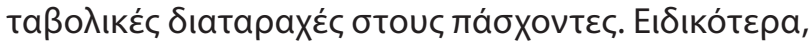

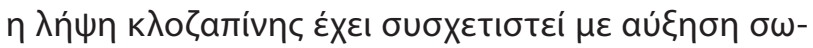

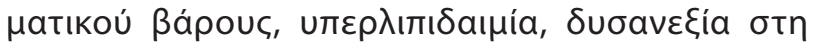

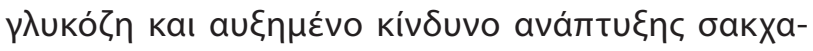

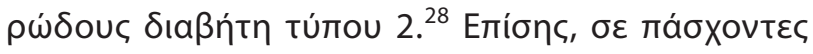

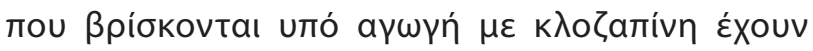

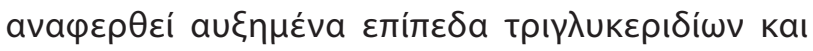

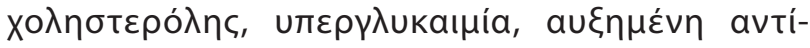

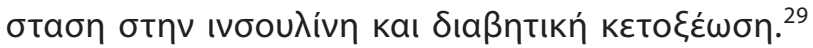

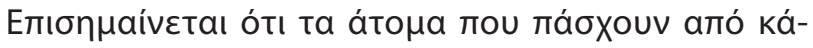

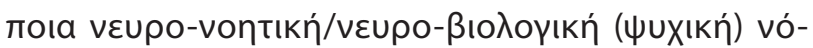

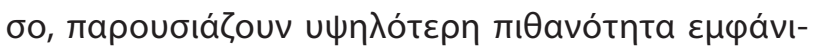

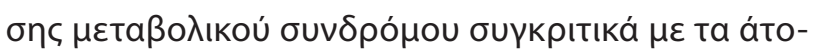

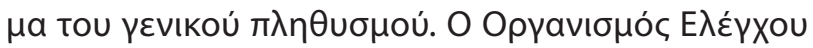

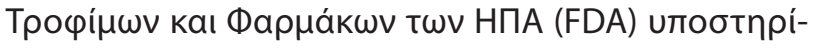

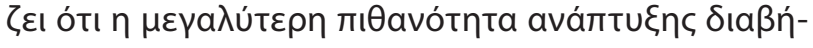

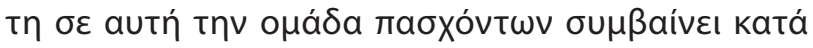

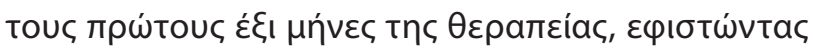

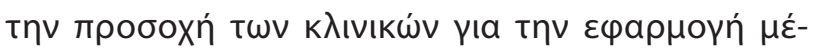

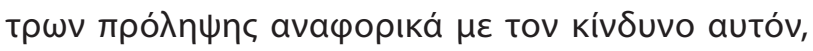

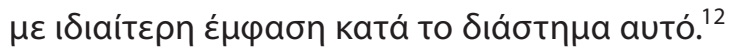

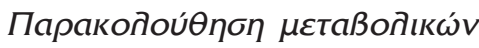

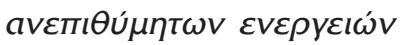

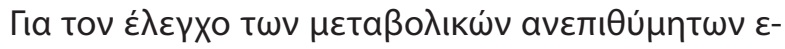

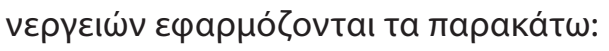

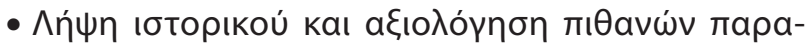

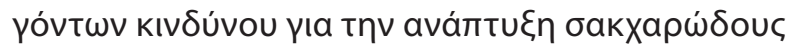




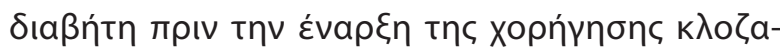

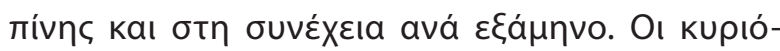

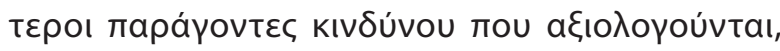

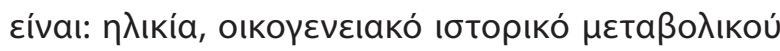

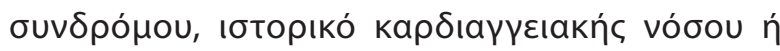

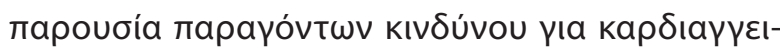

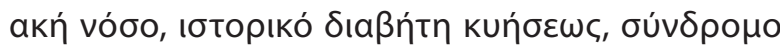

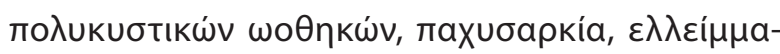

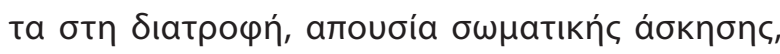
$\varepsilon \theta$ viкótnта. ${ }^{11}$

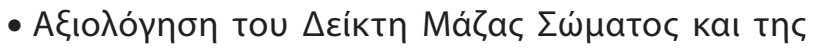

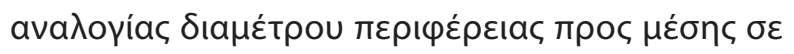

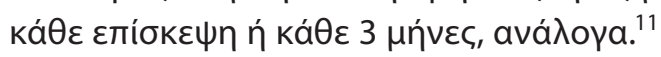

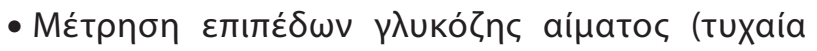

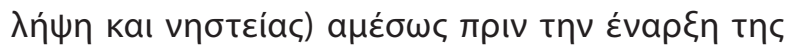

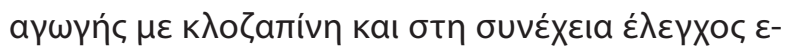

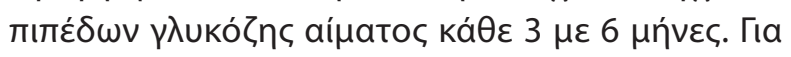

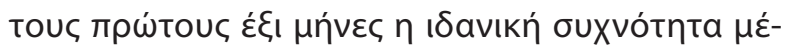

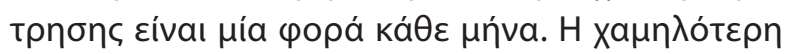

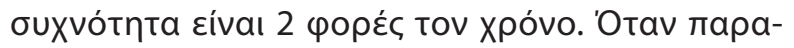

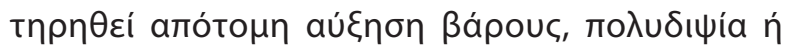

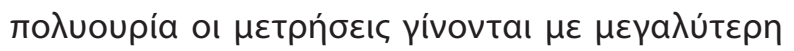
бuxvótnta. ${ }^{11}$

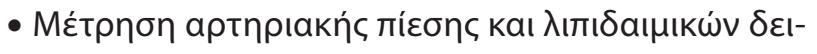

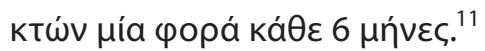

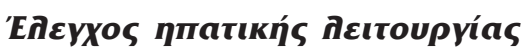

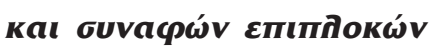

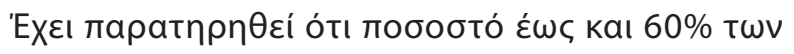

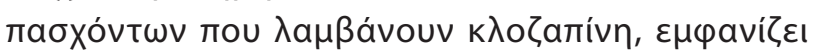

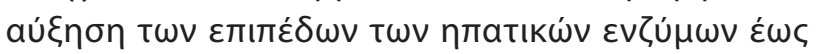

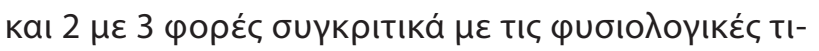

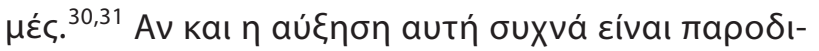

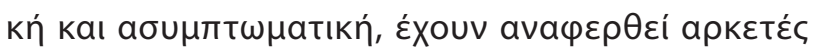

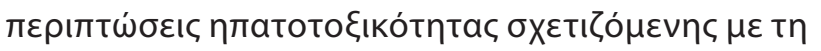

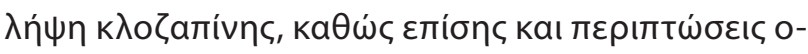

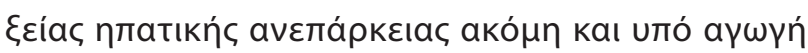

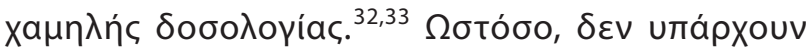

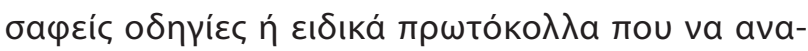

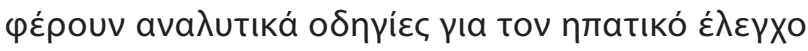

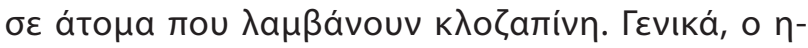

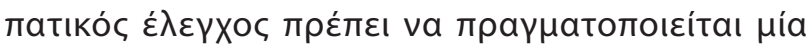

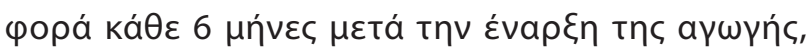

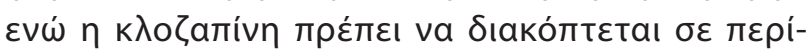

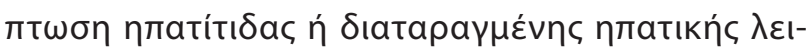
toupyíac. ${ }^{12-14}$

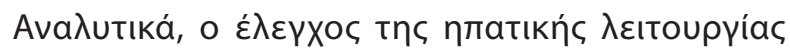

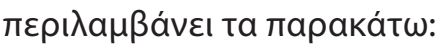

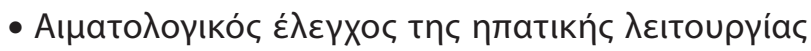

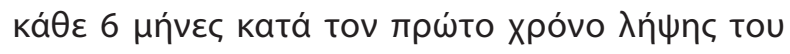

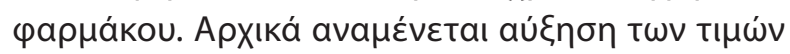

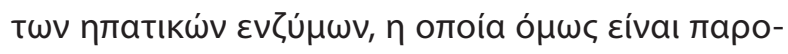

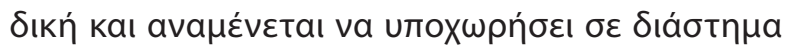

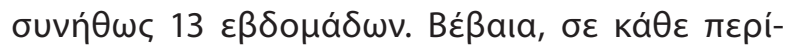

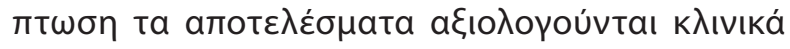

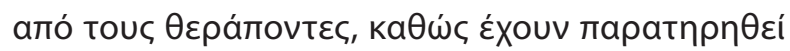

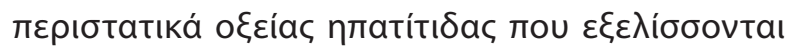

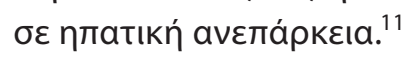

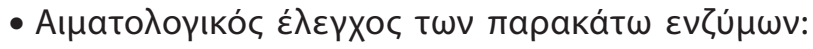

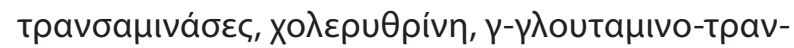

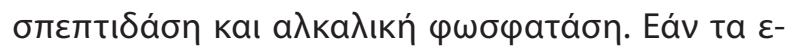

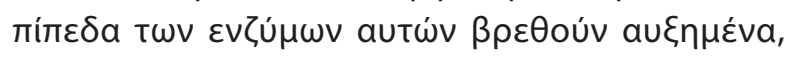

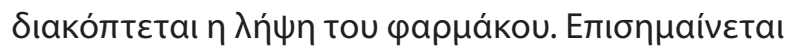

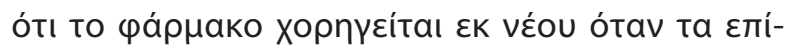

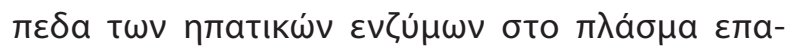

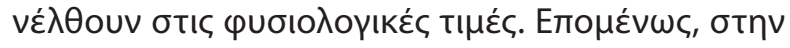

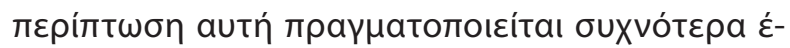

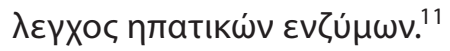

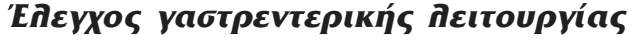

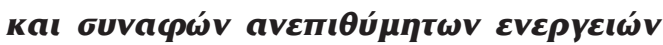

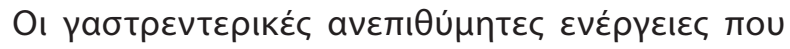

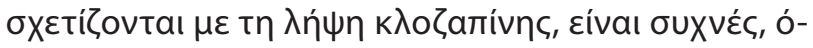

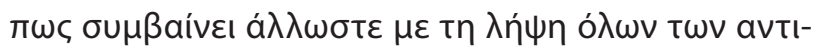

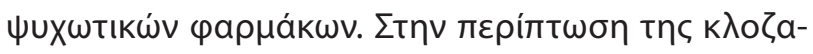

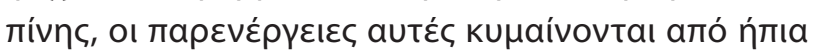

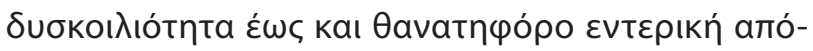

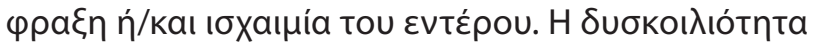

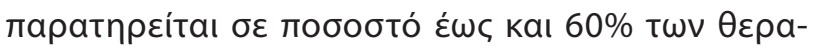

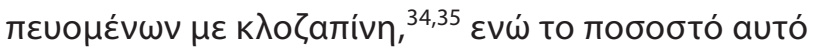

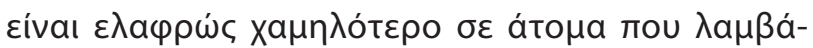

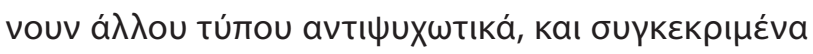

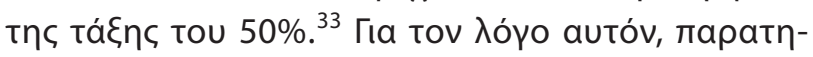

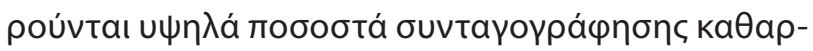

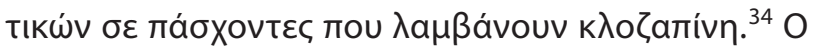

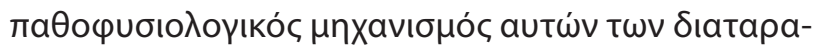

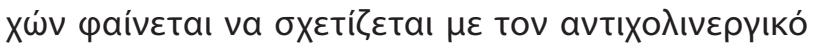

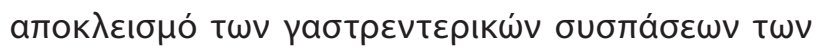

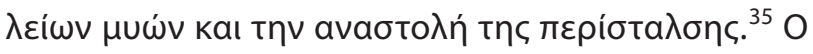

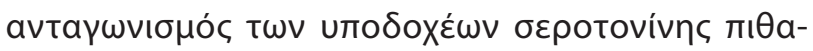

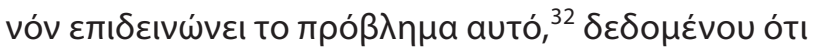

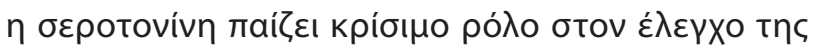




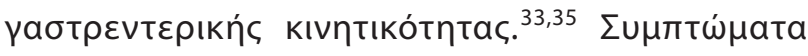

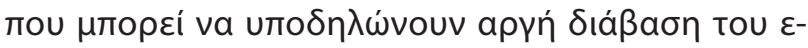

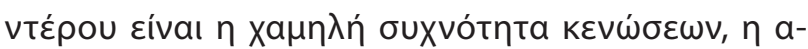

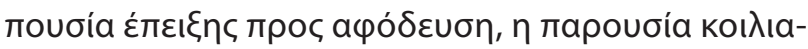

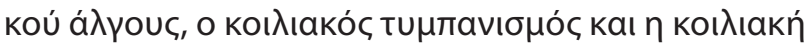

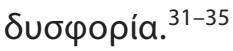

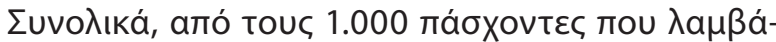

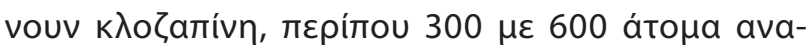

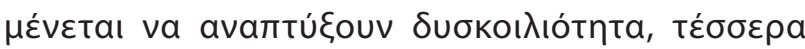

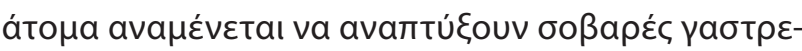

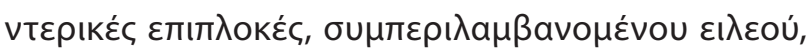

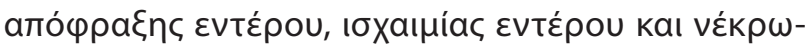

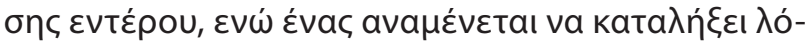

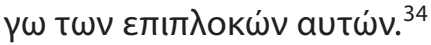

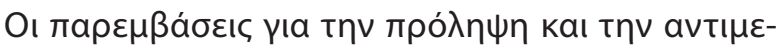

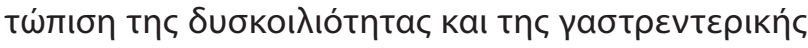

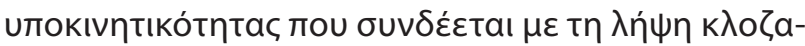

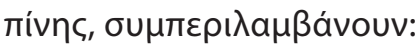

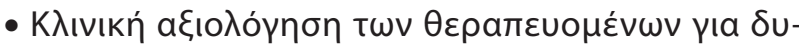

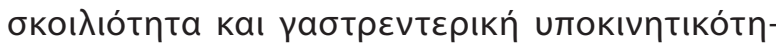

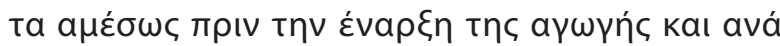

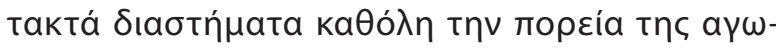
үท́c. ${ }^{11}$

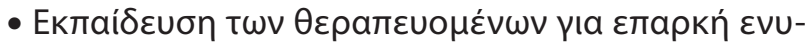

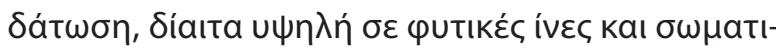
кń áoknon. ${ }^{11}$

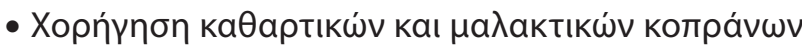

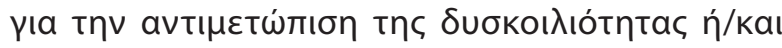

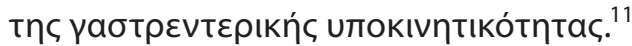

- Етаvakı

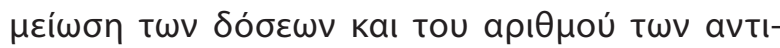

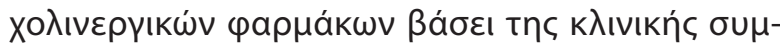

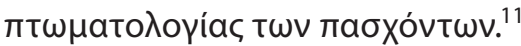

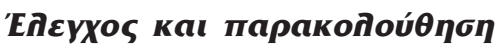

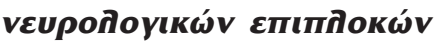

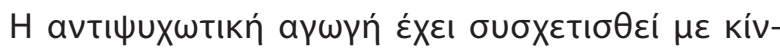

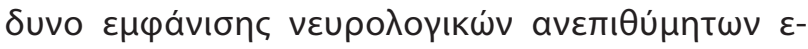

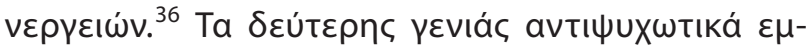

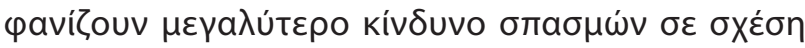

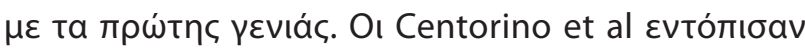

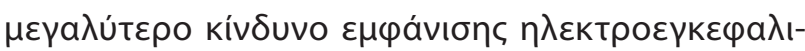

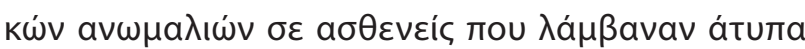

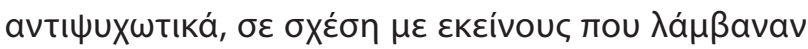

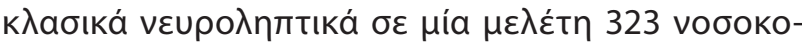

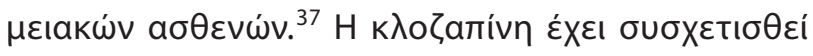

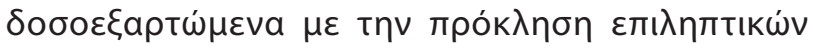

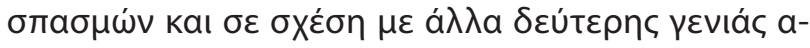

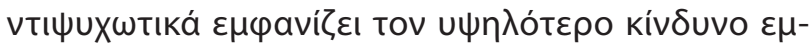
pávionc. $^{38}$

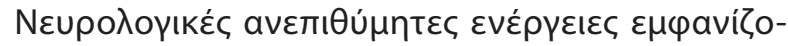

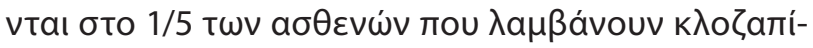

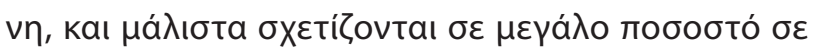

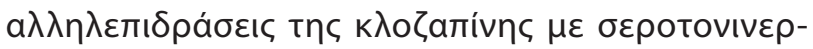

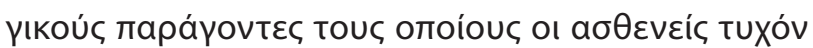
$\lambda a \mu \beta a ́ v o u v$ tautóxpova. ${ }^{36,37}$

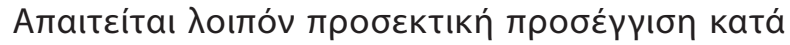

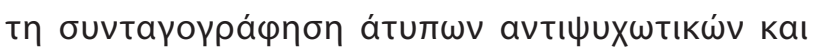

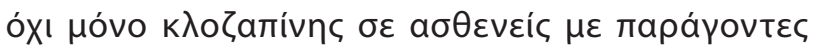

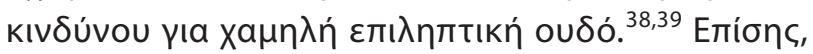

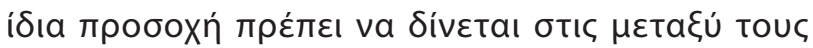

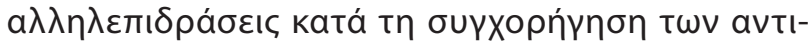

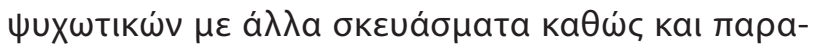

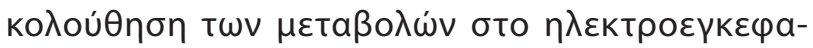

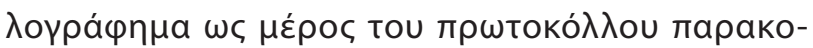
$\lambda$ oúӨnonc. ${ }^{39}$

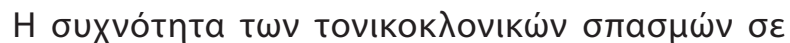

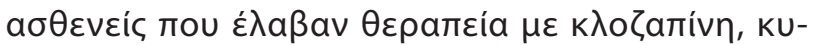

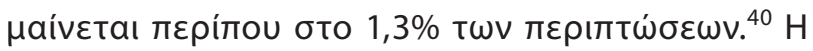

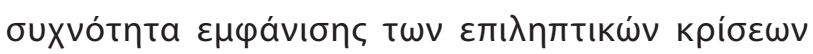

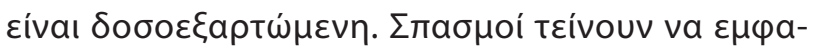

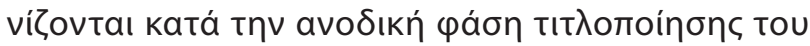

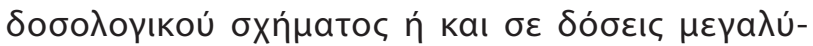

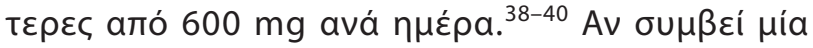

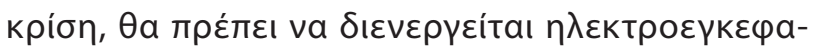

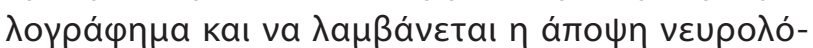
you. ${ }^{40}$

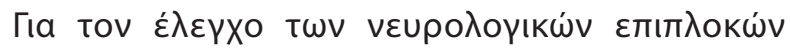

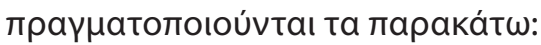

- Праүнатотоínon Н

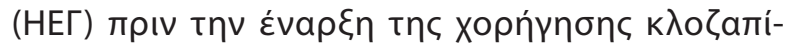
vnc. ${ }^{11,40}$

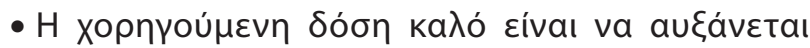

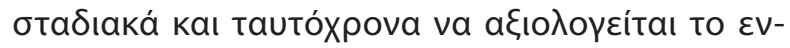

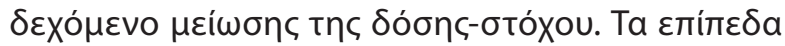

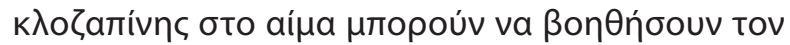

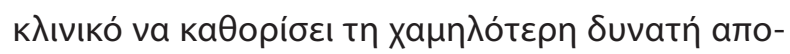

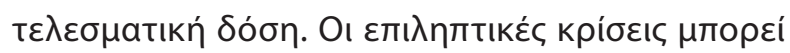

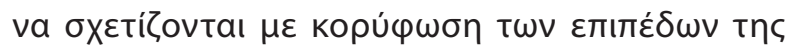

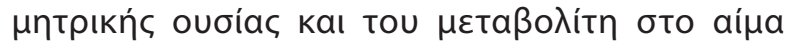




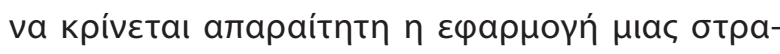

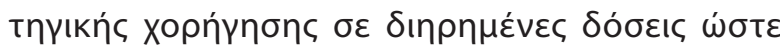

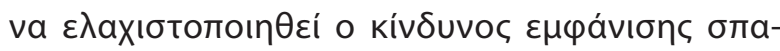
$\sigma \mu \omega \dot{v} .^{11,39}$

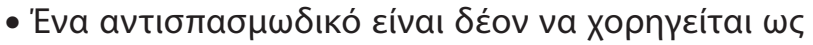

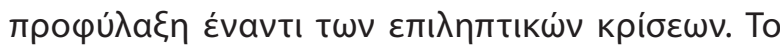

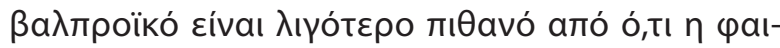

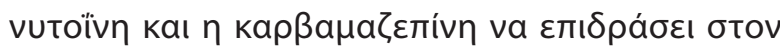

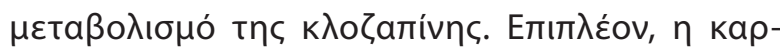

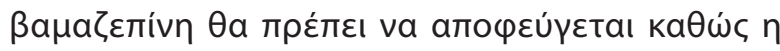

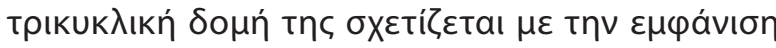

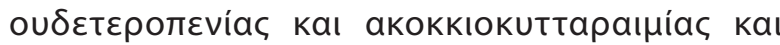

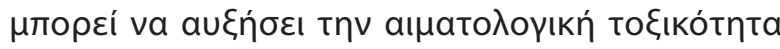

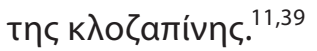

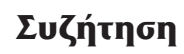

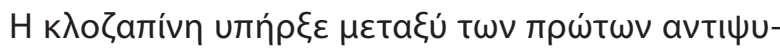

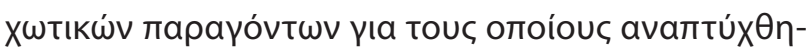

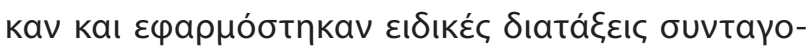

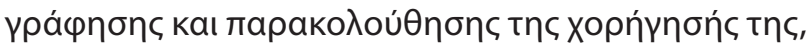

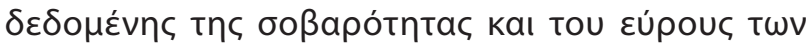

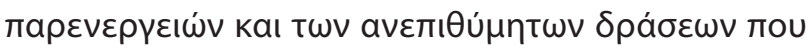

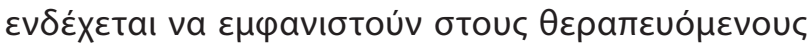

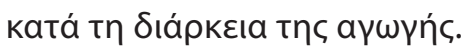

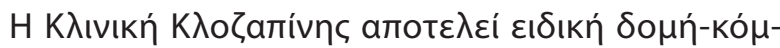

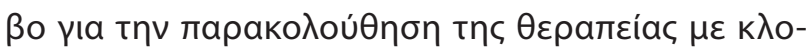

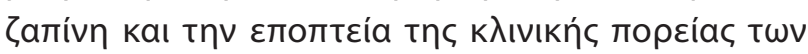

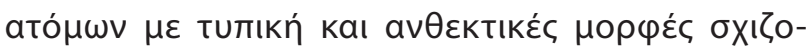

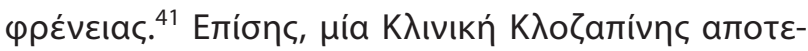

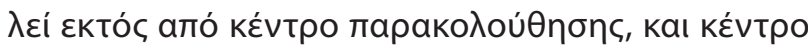

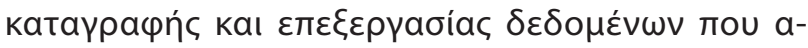

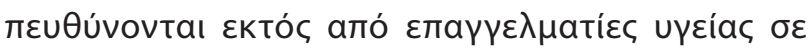

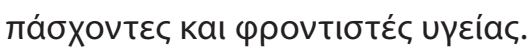

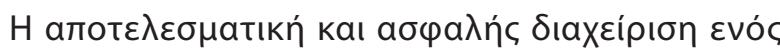

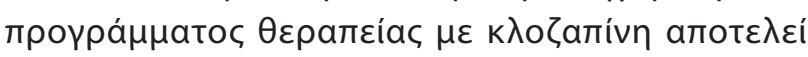

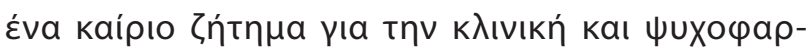

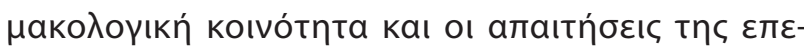

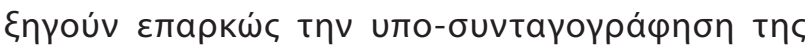

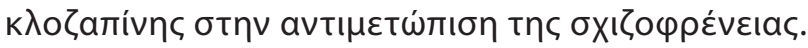

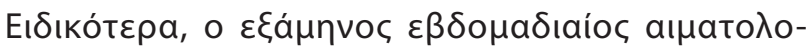

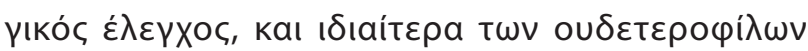

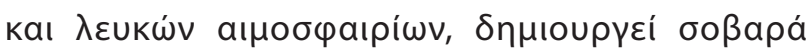

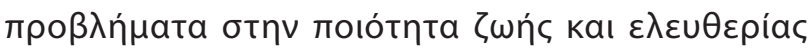

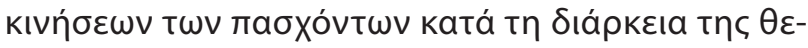

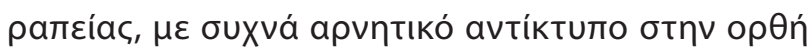

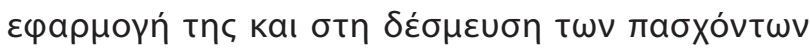
$\sigma^{\prime}$ autri. ${ }^{42}$

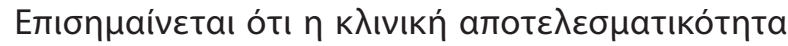

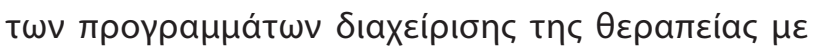

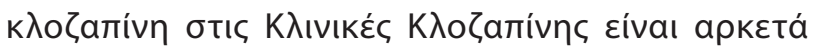

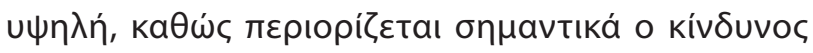

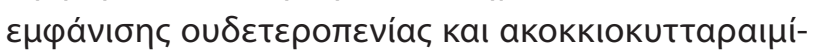

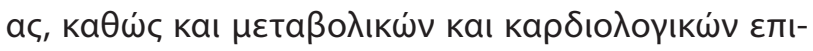

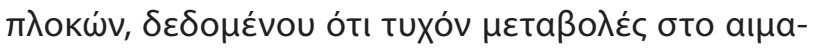

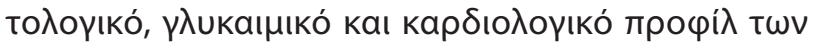

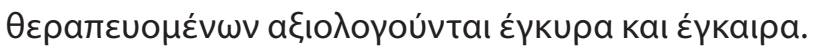

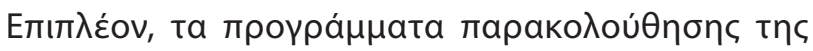

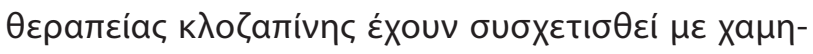

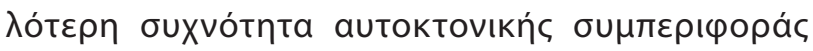

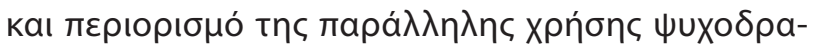

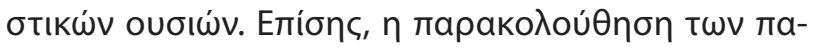

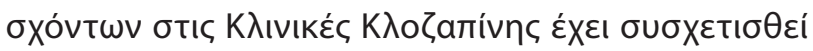

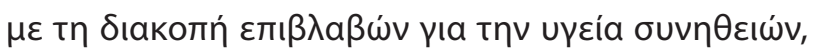

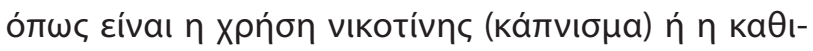

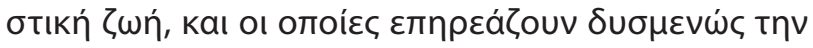

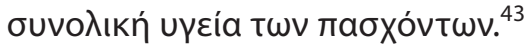

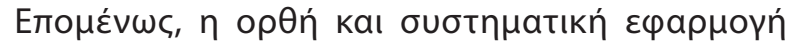

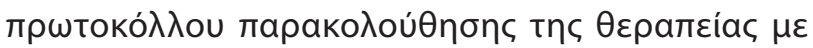

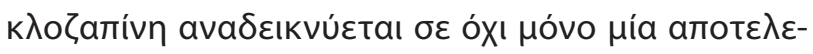

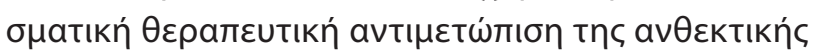

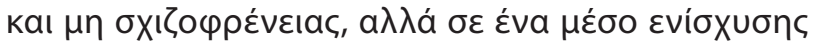

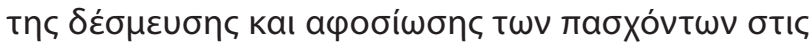

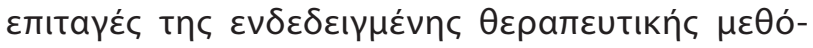
Sou. ${ }^{42,43}$

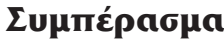

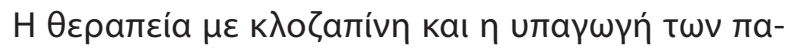

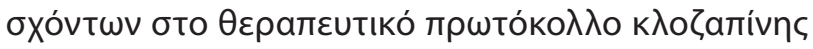

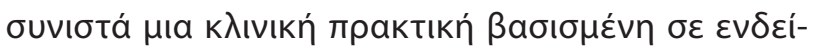

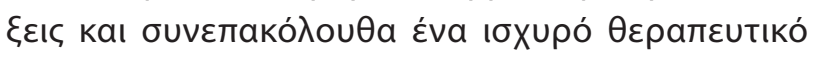

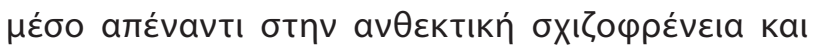

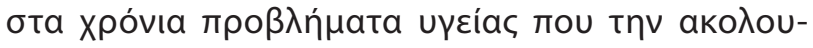

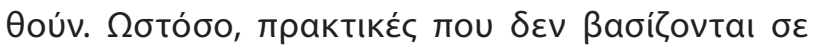

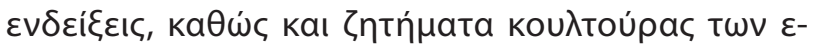

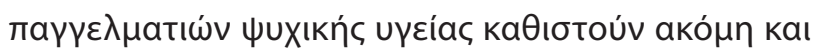

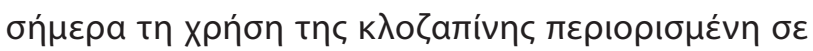

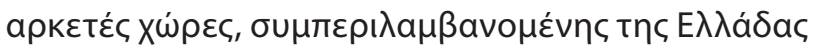
кaı тпৎ Kúnроu. 


\title{
Development and implementation of clozapine protocol in patients with schizophrenia in Greece
}

\author{
M. Nystazaki, ${ }^{1}$ M. Karanikola, ${ }^{2}$ V. Gartzoni, ${ }^{1}$ A. Georgou, ${ }^{3}$ \\ St. Tolia, ${ }^{3}$ Chr. Liapis, ${ }^{1}$ M. Psomiadi, ${ }^{4}$ G. Alevizopoulos ${ }^{1}$ \\ 'Department of Psychiatry, Aghioi Anargyroi Hospital, National \\ and Kapodistrian University of Athens, Athens, Greece, \\ ${ }^{2}$ Department of Nursing, Cyprus University of Technology, Cyprus, \\ ${ }^{3}$ Zografou Mental Health Centre, National and Kapodistrian University of Athens, Athens, \\ ${ }^{4}$ Department of Health Service Management, National School of Public Administration \\ and Local Government, Athens, Greece
}

Psychiatriki 2020, 31:70-81

Clozapine is an atypical antipsychotic used for the treatment of resistant schizophrenia, exhibiting significant advantages over other antipsychotic agents. Clozapine efficacy is well established in people diagnosed with schizophrenia via reducing both positive and negative symptoms. Also, is associated with a low risk of extrapyramidal side effects compared to other antipsychotics. Despite the above, clozapine is an unpopular therapeutic option for patients not previously responded to other antipsychotics, because of adverse side effects, such as hyper-salivation and weight gain or critical side effects, i.e., risk for developing neutropenia and agranulocytosis and the need for a systematic and vigilant patients' monitoring, causing discomfort to them and increased expenses to the healthcare system. The aim of the present article is to describe (a) the development of a "clozapine treatment monitoring protocol", and (b) the monitoring process applied at the Department of Psychiatry of Aghioi Anargyroi Cancer Hospital in patients under clozapine treatment. For the protocol development a systematic review of the existing literature was conducted. An advanced search in Medline, CINAHL, Scopus and Google Scholar was conducted, as well as at the National Organization of Greece for Medicines database, with the following key- words: "clozapine", "clozapine protocol", "clozapine monitoring", "clozapine guidelines". Based on this procedure, the Victorian Consensus View protocol applied in Australia was evaluated as the most appropriate since it encompasses: (a) monitoring of multiple systems based on a holistic healthcare approach towards patients, and (b) Intense cardiovascular functioning monitoring, highly relevant to the Greek population due to increased incidence of myocarditis. Overall, the necessary interventions prior and after clozapine treatment initiation are, monitoring of heamatological and cardiovascular function and related side effects, metabolic monitoring and related side effects, monitoring of metabolic adverse effects, gastrointestinal and neurological adverse effects, hepatic function monitoring and related side effects. Clozapine treatment monitoring protocol applied at special settings, e.g., Clozapine Clinics, is highly beneficial, since the risk of neutropenia, agronulocytosis is minimized, while suicidal behavior and substance use are reduced along with risky health behaviors, i.e., nicotine use and sedentary lifestyle. The current protocol may be applied by mental healthcare professionals aiming to empower individuals with schizophrenia through promoting their independency and quality of life.

Key words: Schizophrenia, clozapine, protocol, side effects. 


\section{Bıßהtoypacpía}

1. Stroup S, Gerhard T, Crystal S, Huang C, Olfson M. Comparative Effectiveness of Clozapine and Standard Antipsychotic Treatment in Adults with Schizophrenia. Am J Psychiatry 2016, 173:166-173, doi/10.1176/appi.ajp.2015.15030332

2. Lieberman JA, Safferman AZ, Pollack S, Szymanski S, Johns C, Howard $A$ et al. Clinical effects of clozapine in chronic schizophrenia: response to treatment. Am J Psychiatry 1994, 151: 1744-1745, doi: 10.1176/ajp.151.12.1744

3. Mortimer A. Using clozapine in clinical practice. Adv Psych Tr 2011, 17:256-265, doi: 10.1192/apt.bp.110.008136

4. Krivoy A, Malka L, Weizman A, Vaevski A. Predictors of Clozapine Discontinuation in Patients with Schizophrenia. Int Clin Psychopharmacol 2011, 26:311-315, doi 10.1097/YIC 0b013e32834ab34c.

5. Samara M, Leucht S. Clozapine in Treatment Resistant Schizophrenia. B J Psych 2017, 210:299, doi: 10.1192/bjp.210. 4.299

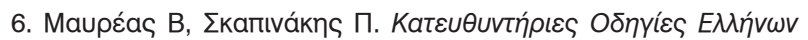

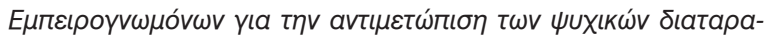

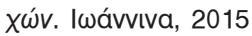

7. Clark SR, Wilton L, Baune BT, Procter N, Hustig H. A statewide quality improvement system utilising nurse-led clinics for clozapine management. Austr Psychiatry 2014, 22:254-259, doi: $10.1177 / 1039856214533395$

8. Warnez S, Alessi-Serevini A. Clozapine: a review of clinical practice guidelines and prescribing trends. $B M C$ Psychiatry 2014, 14:102, doi: 10.1186/1471-244X-14-102

9. Nikolaou M, Miliopoulos V, Lazaros G, Karavidas A, Trikas A, Karvounis $\mathrm{CH}$ et al. Diagnosis and Management of Myocarditis: Data from HERMES- the Hellenic Registry on Myocarditis Syndromes. Eur Heart J 2017, 38:1, doi: 10.1093/eurheartj/ehx504. P3519

10. Lehman AF, Lieberman JA, Dixon LB, McGlashan TH, Miller AL, Perkins DO et al. American Psychiatric Association, Steering Committee on Practice Guidelines: Practice Guidelines for the treatment of patient with schizophrenia, second edition. Am J Psychiatry 2004, 161:1-56, PMID: 15000267

11. Berk M, Fitzimons J, Lambert T, Pantelis C, Kulkarni J, Castle $\mathrm{D}$ et al. Monitoring the safe use of clozapine, a consensus view from Victoria, Australia. CNS Drugs 2007, 21:117-127, doi: 10.2165/00023210-200721020-00003

12. Sultan SR, Olfson M, Correll UC, Duncan JE. Evaluating the Effect of the Changes in FDA Guidelines for Clozapine Monitoring. J Clin Psychiatry 2017, 78:e933-e939, doi: 10.4088/ JCP. $16 \mathrm{~m} 11152$

13. Taylor D,Paton C,Kapur S. The Maudsley Prescribing Guidelines. 12th edition, InformaHealthcare, London, 2015

14. Barnes RET. Evidence-based guidelines for the pharmacological treatment of schizophrenia: recommendations from the British Association for Psychopharmacology. J Psychopharmac 2011, 0:1-54, doi: 10.1177/0269881110391123

15. Gerson SL. G-CSF and the management of clozapine-induced agranulocytosis. J Clin Psychiatry 1994, 55:139-42, PMID: 7525542
16. Alvir JM, Lieberman JA, Safferman AZ, Schwimmer JL, Schaaf JA. Clozapine-induced agranulocytosis. Incidence and risk factors in the United States. N Engl J Med 1993, 329:162-167, doi:10.1056/NEJM199307153290303

17. Balda MV, Garay OU, Papale RM, Bignone I, Bologna VG, Brandolini A et al. Clozapine-associated neutropenia and agranulocytosis in Argentina (2007-2012). Int Clin Psychopharmacol 2015, 30:109-114, doi: 10.1097/YIC.0000000000000060.

18. Legge ES, Hamshere M, Hayes DR, Dowsns J, O' Donovan MC, Owen MJ et al. Reasons for discontinuing clozapine: A cohort study of patients commencing treatment. Schizophr Res 2016, 174:113-119, doi:10.1016/j.schres.2016.05.002

19. Capllonch A, de Pablo S, de la Torre A, Morales I. Increase in white cell and neutrophil counts during the first eighteen weeks of treatment with clozapine in patients admitted to a long-term psychiatric care inpatient unit. Rev Psiquiatr Salud Ment 2016, 11:94-100, doi: 10.1016/S2215-0366(14)70245-7

20. Ronaldson JK, Fitzgerald BP, Taylor JA, Topliss DJ, McNeil JJ. A new monitoring protocol for clozapine-induced myocarditis based on an analysis of 75 cases and 94 controls. Aust N Z J Psychiatry 2011, 45:458-465, doi: 10.3109/00048674.2011.572852

21. Layland J, Liew D, Prior D. Clozapine-included cardiotoxicity a clinical update. Med J Aust 2009, 16:190-192, PMID: 19220183

22. Khan AA, Ashraf A, Baker D, Al-Omary MS, Savage L, Ekmejian $A$ et al. Clozapine and incidence of myocarditis and sudden death - Long term Australian experience. Int J Cardiol 2017, 238:136-139, doi: 10.1016/j.ijcard.2017.03.013

23. Kontoangelos K, Loizos S, Kanakakis J, Smyrnis N, Economou M, Bergiannaki JD et al. Myocarditis after administration of Clozapine. Eur Rev Med Pharmacol Sci 2014, 18:2383-286, PMID: 25219841

24. Datta T, Solomon A. Clozapine-induced myocarditis. Oxf. Med. Case Reports 2018, 1, doi: 10.1093/omcr/omx080

25. Bellissima BL, Tingle MD, Cicović A, Alawami M, Kenedi C. A systematic review of clozapine-induced myocarditis. Int $J$ Cardiol 2018, 15:122-129, doi: 10.1016/j.ijcard.2017.12.102

26. Merrill D, Ahmari SE, Bradford JM, Lieberman JA. Myocarditis During Clozapine Treatment. Am J Psychiatry 2006, 163:204208, doi: 10.1176/appi.ajp.163.2.204

27. Rostagno C, Domenichetti S, Pastorelli F, Gensini GF. Clozapine associated cardiomyopathy: a cluster of 3 cases. Intern Emerg Med 2011, 6:281-283, doi: 10.1007/s11739-010-0468-4

28. Hert M, Detraux J, Van Winkel R, Yu W, Correll CU. Metabolic and cardiovascular adverse effects associated with antipsychotic drugs. Nat Rev Endocrinol 2012, 18:114-126, doi: 10.1038/ nrendo.2011.156.

29. Ucok A, Gaebel W. Side effects of atypical antipsychotics: a brief overview. World Psychiatry 2008, 7:58-62, doi: 10.1002/ j.2051-5545.2008.tb00154.x

30. Gaertner HJ, Fischer E, Hoss J. Side effects of clozapine. Psychopharmacol 1989, 99:97-100, doi: 10.1007/BF 00442570 
31. Hummer M, Kurz M, Kurzthaler I, Oberbauer $H$, Miller C, Fleischhacker WW. Hepatotoxicity of clozapine. J Clin Psychopharmacol 1997,17:314-317, PMID: 9241012

32. Macfarlane B, Davies S, Mannan K, Sarsam R, Pariente D, Dooley J. Fatal acute fulminant liver failure due to clozapine: a case report and review of clozapine-induced hepatotoxicity. Gastroenterol 1997,112:1707-1709, PMID: 9136851

33. Kellner M, Wiedemann K, Krieg JC, Berg PA. Toxic hepatitis by clozapine treatment. Am J Psychiatry 1993, 150:985-986, doi: 10.1176/ajp.150.6.985b

34. Hayes G, Gibler B. Clozapine-induced constipation. Am J Psychiatry 1995, 152:298a-298, doi: 10.1016/j.ebiom.2016.02. 037

35. Ozbilen M, Adams CE. Systematic overview of Cochrane reviews for anticholinergic effects of antipsychotic drugs. J Clin Psychopharmacol 2009, 29:141-146, doi: 10.1097/ JCP.0b013e31819a91f1

36. Jackson A, Senevirante U. EEG Changes in Patients on Antipsychotic Therapy: a Systematic Review. Epilepsy Behav 2019, 95:1-9, doi: 10.1016/j.yebeh.2019.02.005

37. Centorrino F, Price BH, Tuttle M, Bahk WM, Hennen J, Albert MJ et al. EEG Abnormalities During Treatment with Typical and Atypical Antipsychotics. Am J Psychiatry 2002, 159:109-115, doi: 10.1176/appi.ajp.159.1.109

38. Mauri MC, Paletta S, Maffini M, Colasanti A, Dragogna F, Di Pace $C$ et al. Clinical pharmacology of atypical antipsychotics: an update. EXCLI J 2014, 13:1163-1191, doi: 10.17877/ DE290R-7037

39. Kohlrausch FB. Pharmacogenetics in schizophrenia: a review of clozapine studies. Bras Psiquiat 2013, 35:305-317, doi. 10.1590/1516-4446-2012-0970
40. Pacia SV, Devinsky O. Clozapine-related seizures: experience with 5,629 patients. Neurology 1994, 44:2247-2249, doi: 10.1212/wnl.44.12.2247

41. Kar N, Barreto S, Chandavarkar R. Clozapine Monitoring in Clinical Practice: Beyond the Mandatory Requirement. Clin Psychopharmacol Neurosci 2016, 14:323-329, doi: 10.9758/ cpn.2016.14.4.323

42. Jabbar F, Casey P, Schelten SL. Kelly BD. What Do You Think of Us? Evaluating Patient Knowledge of and Satisfaction with a Psychiatric Outpatient Service. Ir J Med Sci 2011, 180:195-201, doi: 10.1007/s11845-010-0504-4

43. Freeman D, Oyewumi L. Will Routine Therapeutic Drug Monitoring Have a Place in Clozapine Therapy? Clin Pharmacokinetics 1997, 32:93-100, doi:10.2165/00003088-199732020-00001

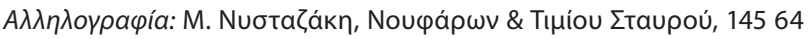

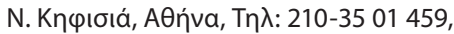

e-mail: manystazaki@nurs.uoa.gr 


\title{
Eıठıкó á $\theta$ $\theta$ o Special article
}

\section{Eupwாaïkń Kotvń $\Delta$ páon:

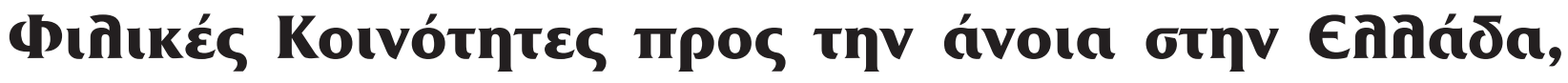

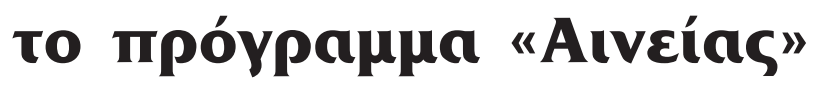

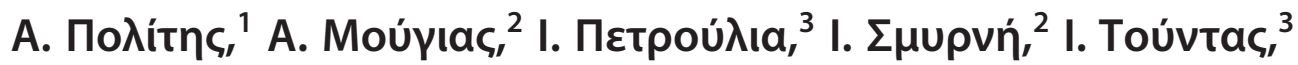

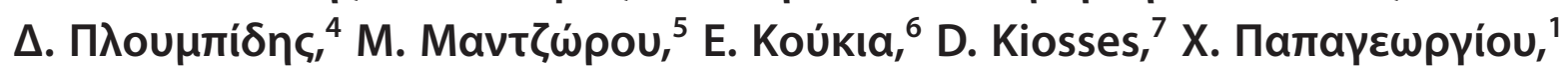

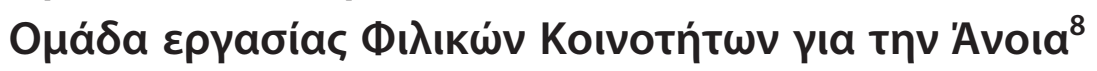

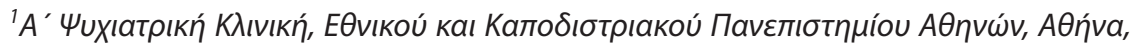

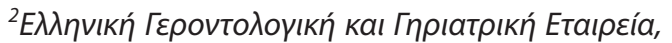

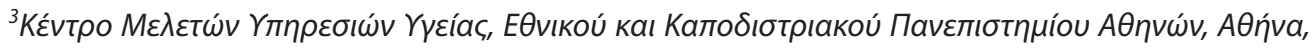

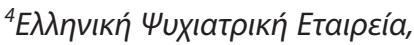

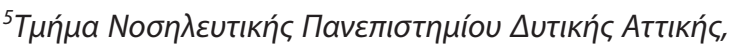

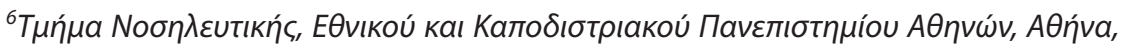 \\ ${ }^{7}$ Weill Cornell Institute of Geriatric Psychiatry, New York, USA,

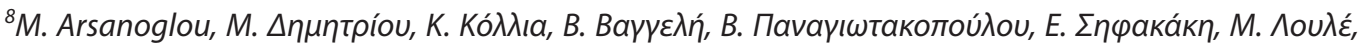

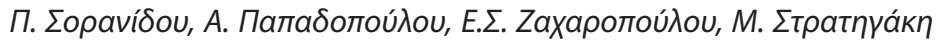

Uuxıatpıkń 2020, 31:82-90

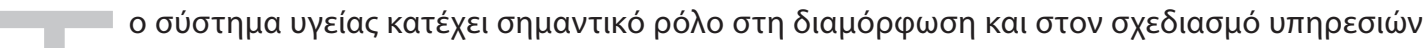

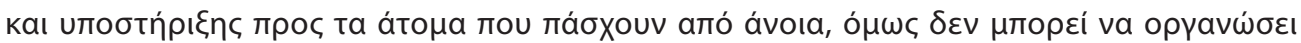

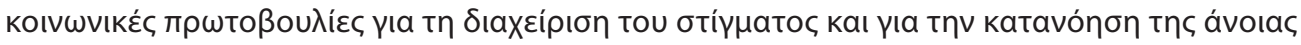

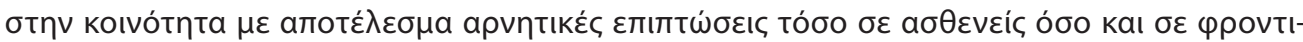

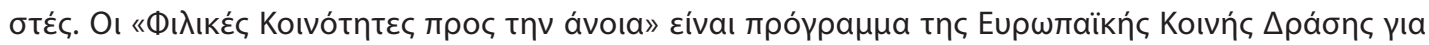

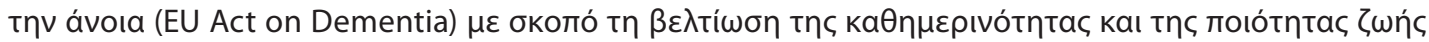

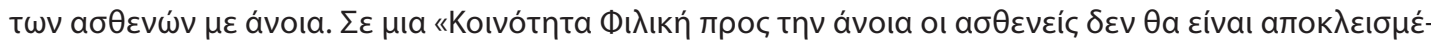

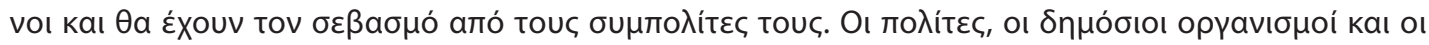

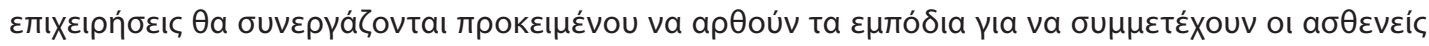

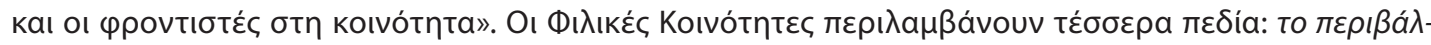

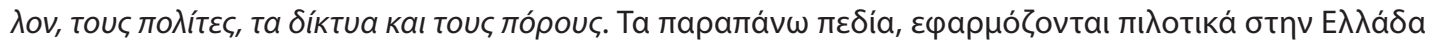




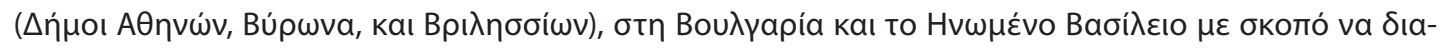

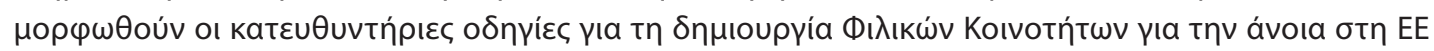

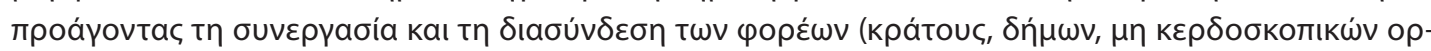

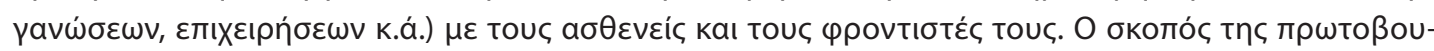

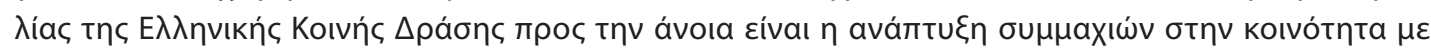

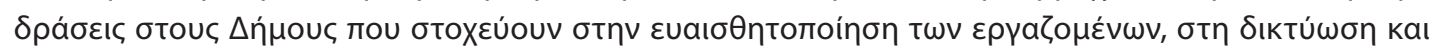

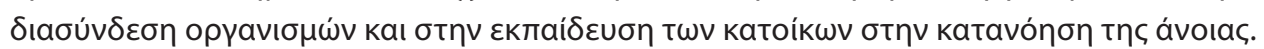

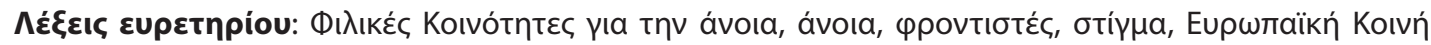
$\Delta$ páon.

\section{Eıбaywyń}

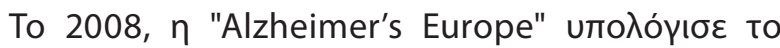

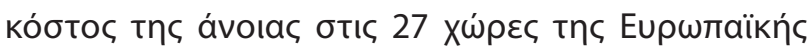

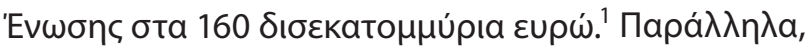

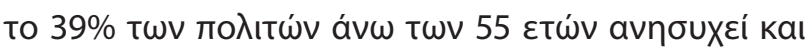

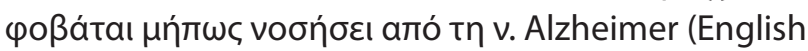

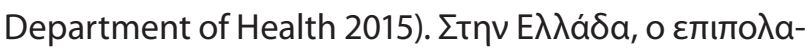

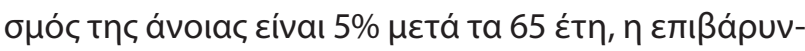

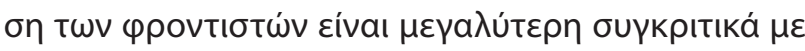

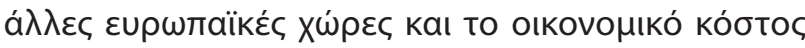

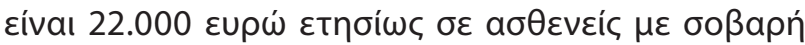

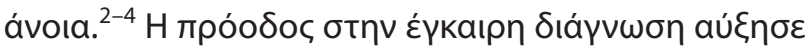

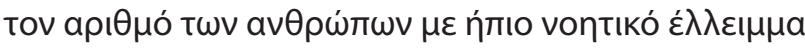

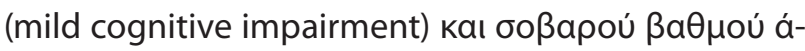

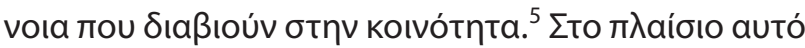

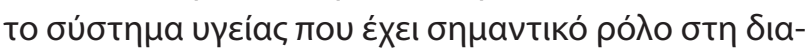

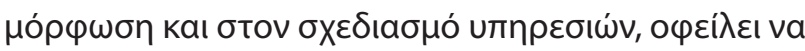

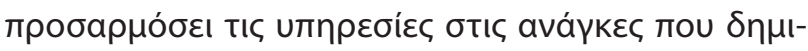

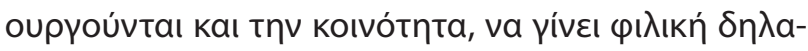

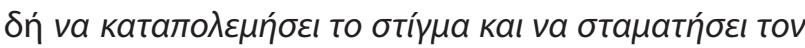

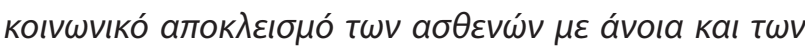

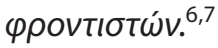

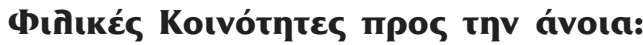

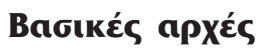

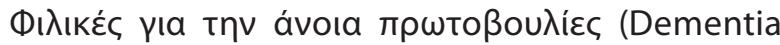

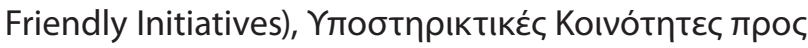

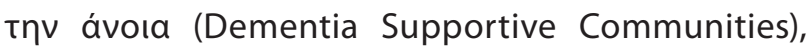

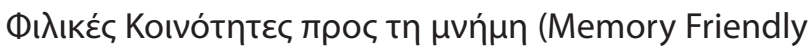

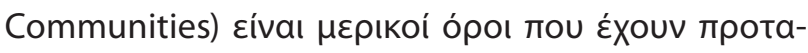

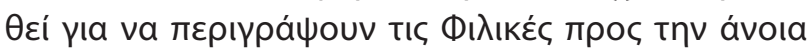

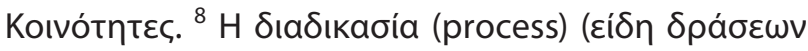

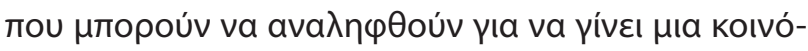

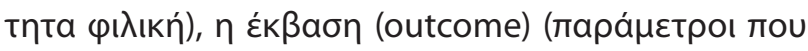

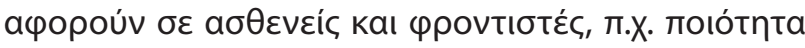

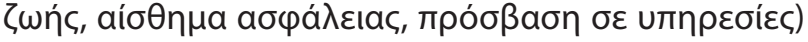

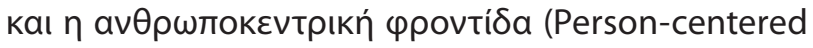

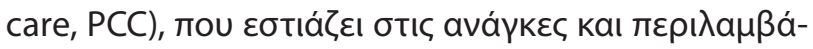

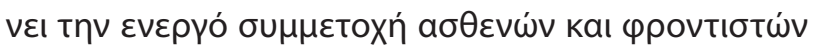

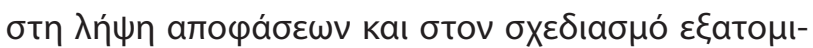

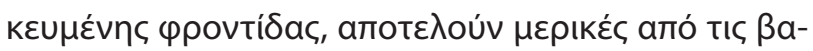

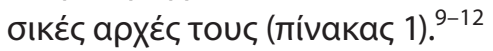

\section{H Eupwriäkń Kotvń $\Delta$ páon yı tnv ávoıa}

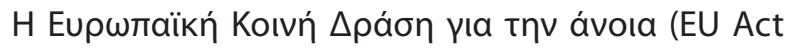

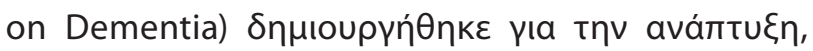

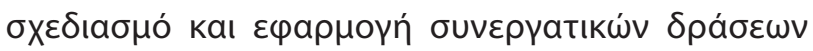

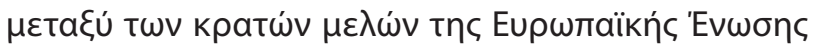

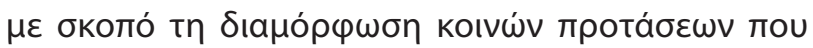

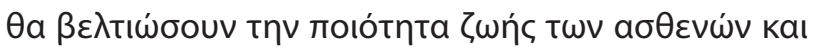

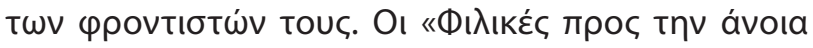

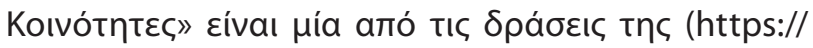
www.actondementia.eu).

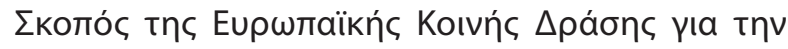

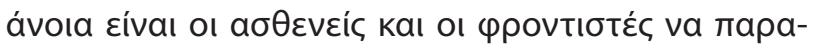

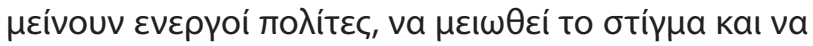

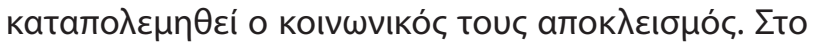

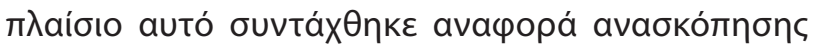

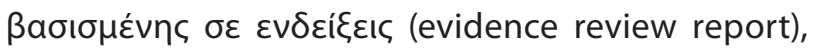

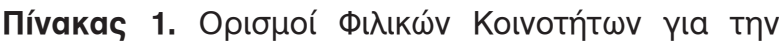
ávoı́. ${ }^{13}$

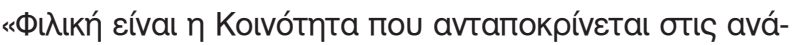

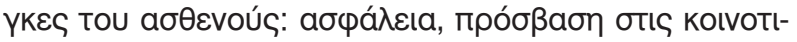

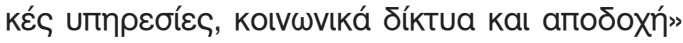

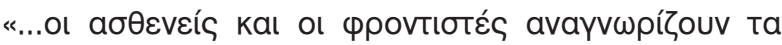

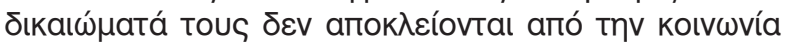

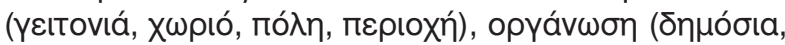

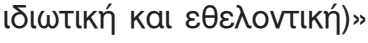




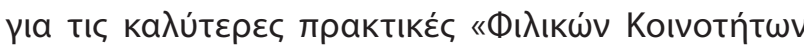

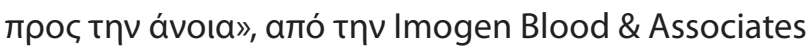

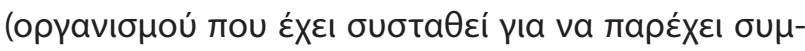

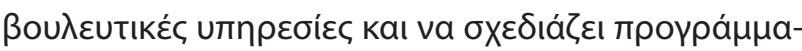

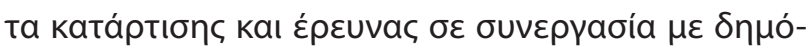

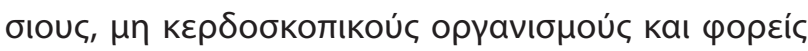

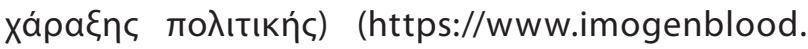

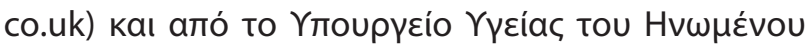

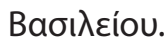

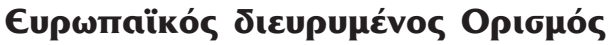

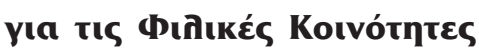 (Europe-wide definition)}

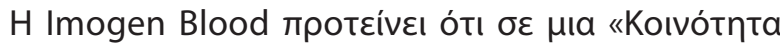

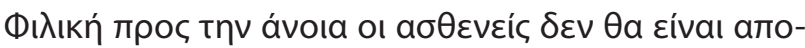

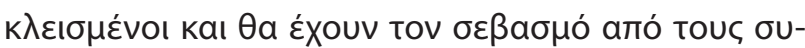

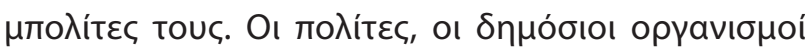

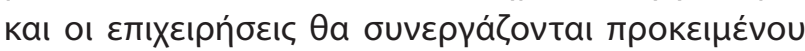

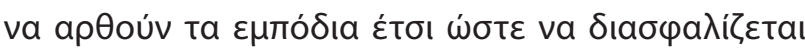

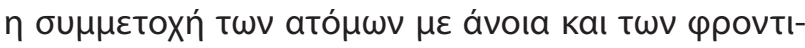

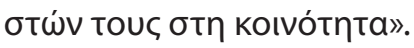

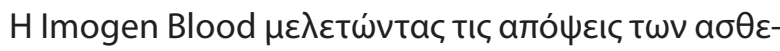

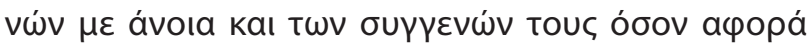

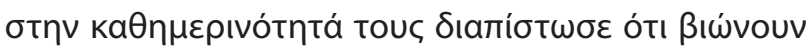

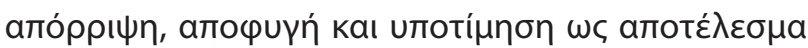

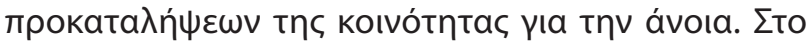

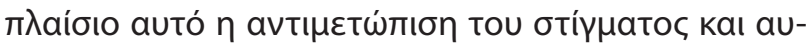

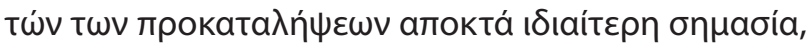

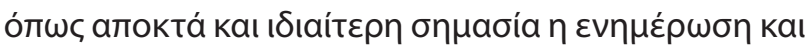

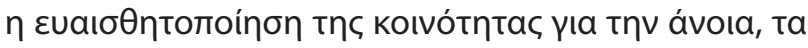

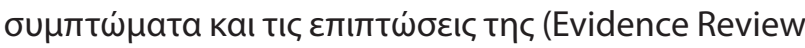
of Dementia Friendly Communities European Union Joint Action on Dementia 2017).

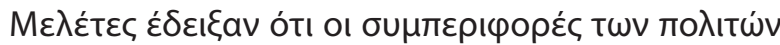

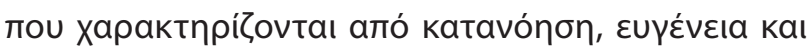

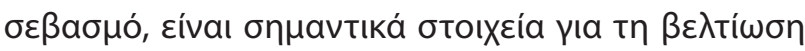

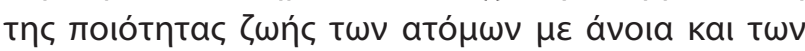

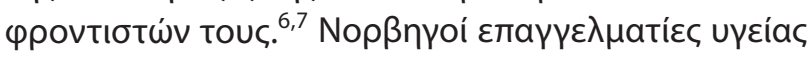

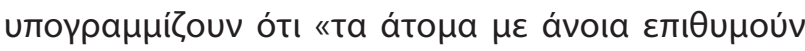

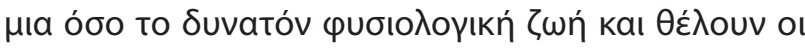

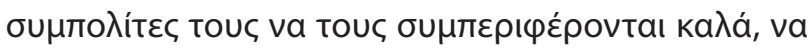

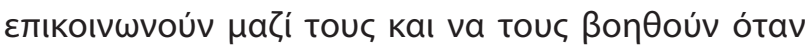
каı óтоu Хрєı́́டદтаı».

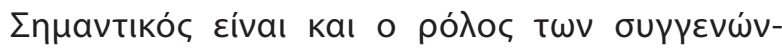

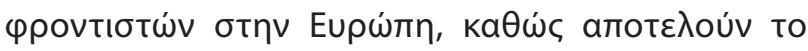

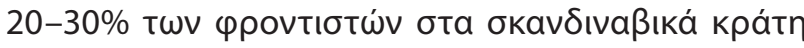

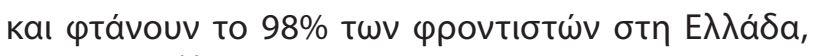

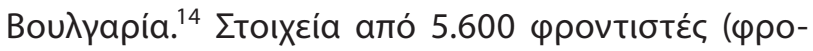

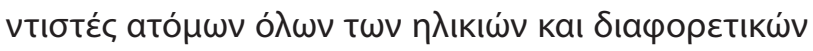

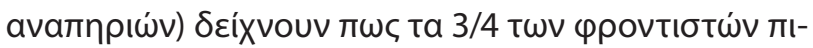

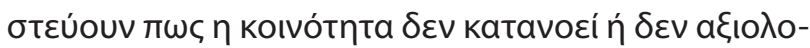

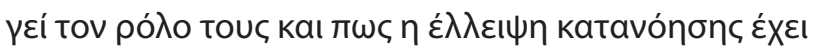

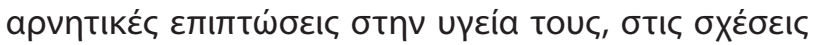

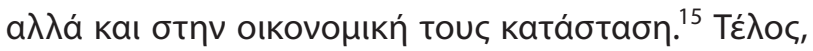

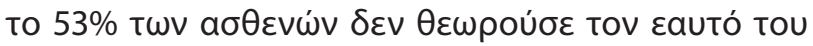

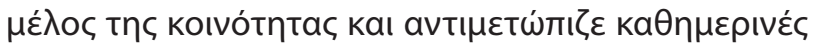

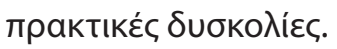

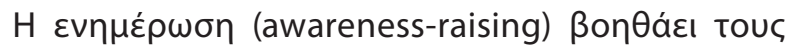

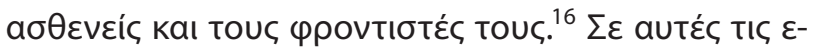

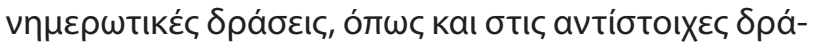

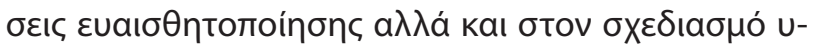

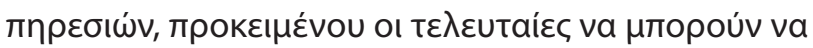

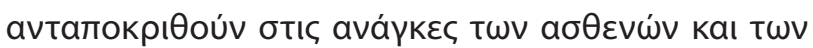

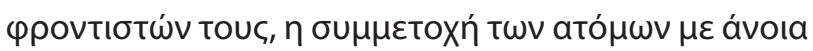

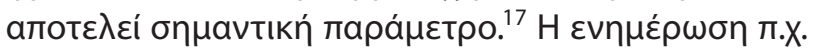

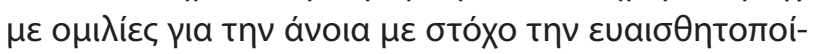

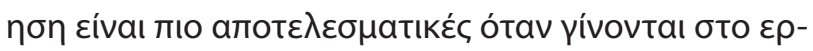

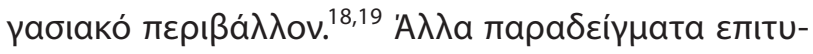

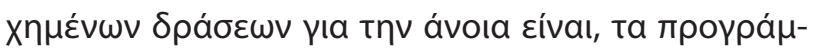

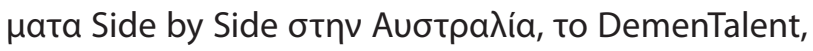

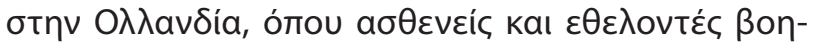

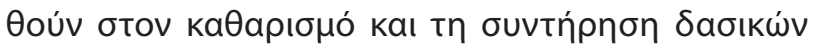

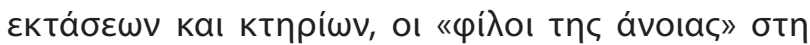

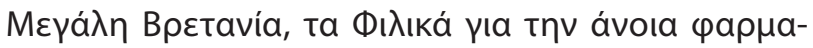

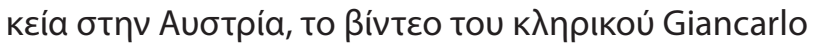

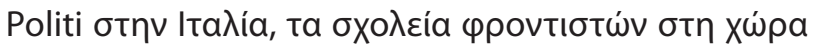

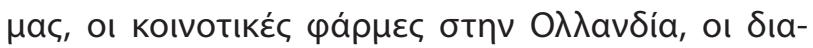

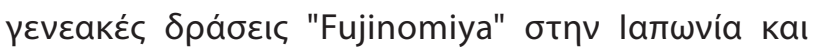

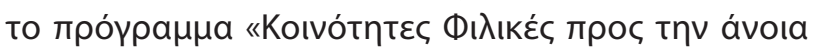

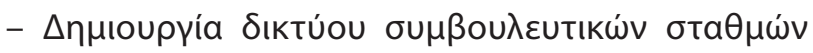

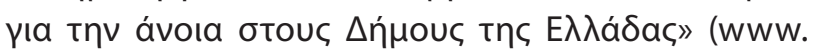
dementia-community.gr) tnৎ Eтaıpcíac Alzheimer AӨnvúv. ${ }^{20-22}$

\section{Про́тumo Фเกิкผ́v \\ mpos tnv ávota Kotvotńtwv}

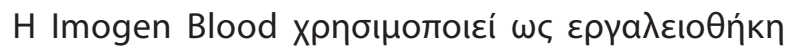

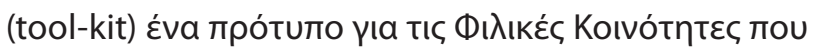

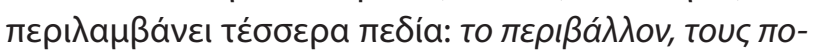

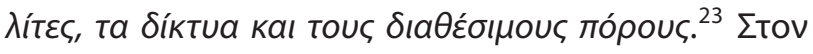

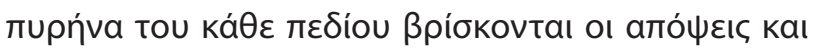

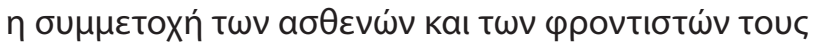
(пívakac 2). 


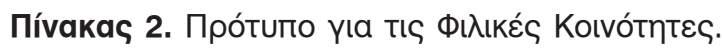

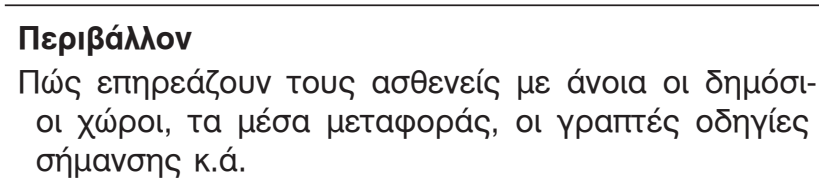

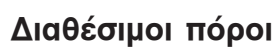

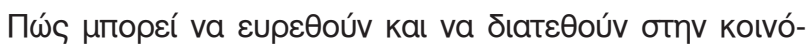

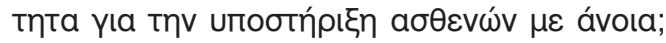

\section{ПоАі́теs}

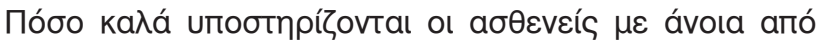

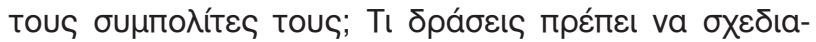

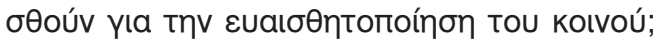

\section{¿íktua}

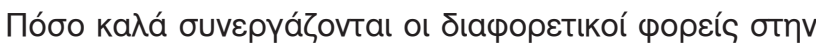

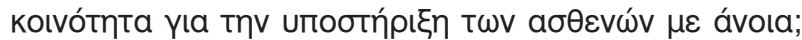

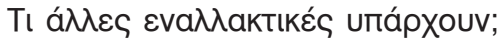

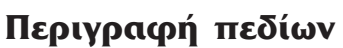

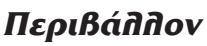

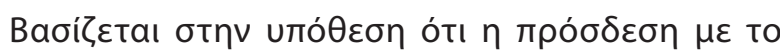

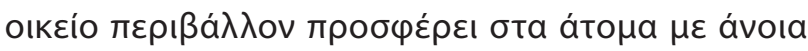

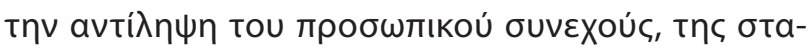

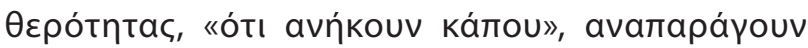

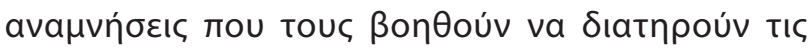

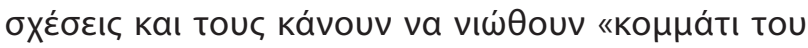

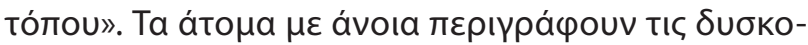

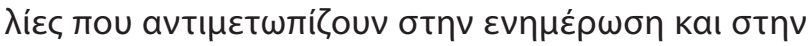

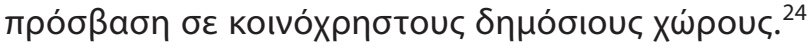

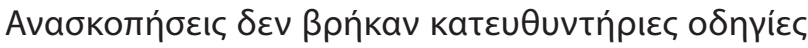

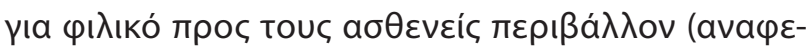

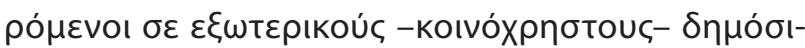

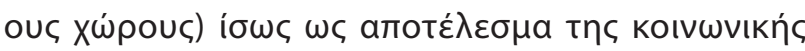

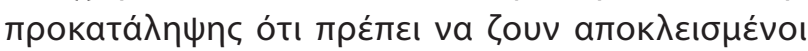

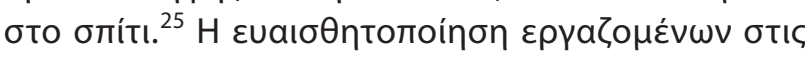

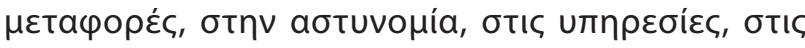

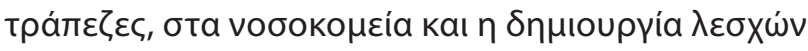

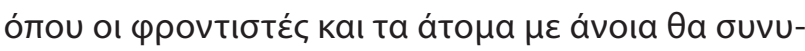

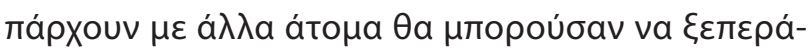

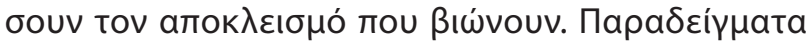

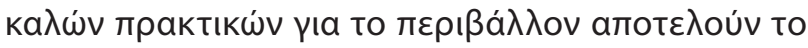

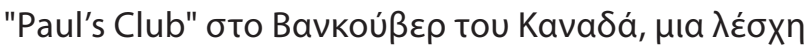
$\mu \varepsilon \tau a \xi u ́ v ~ v \varepsilon ́ \omega v ~ a \sigma \theta \varepsilon v \omega ́ v ~ \mu \varepsilon$ ávoı́ kaı $\varepsilon \theta \varepsilon \lambda o v \tau \omega ́ v$, to

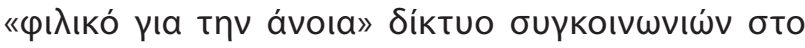

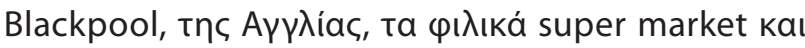

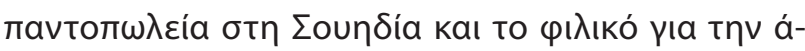

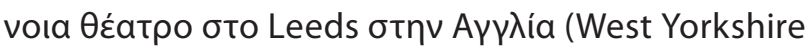
Playhouse).

\section{Мíктua}

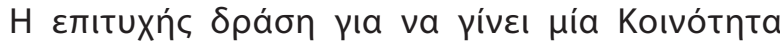

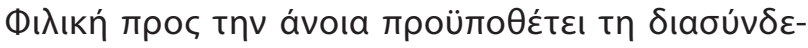

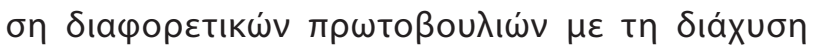

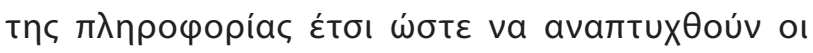

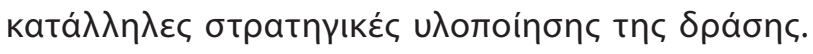

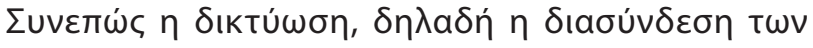

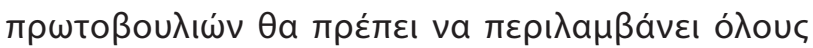

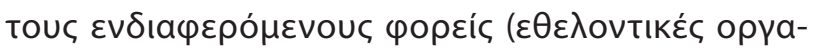

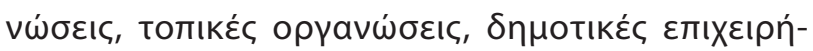

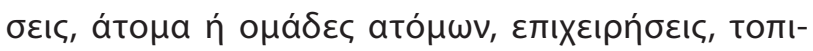

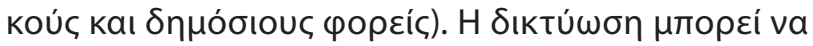

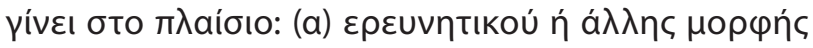

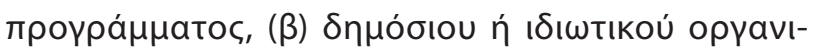

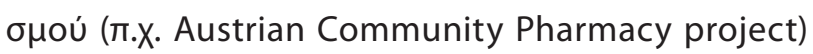

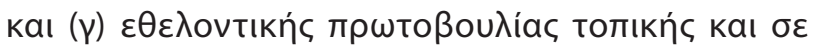

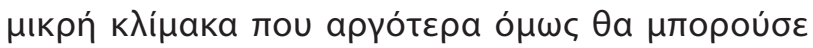

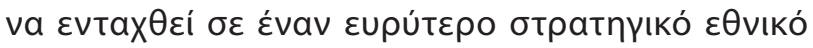

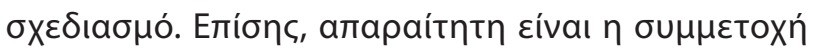

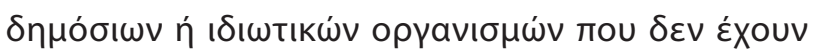

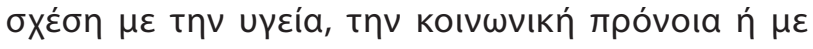

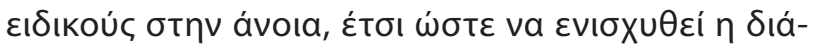

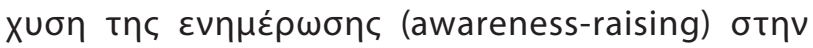

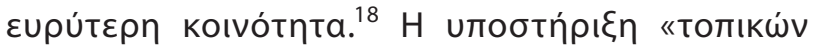

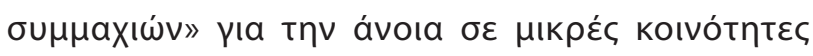

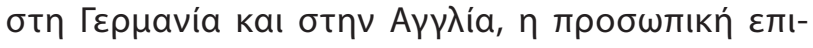

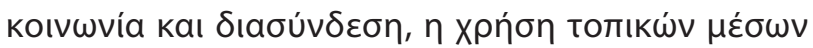

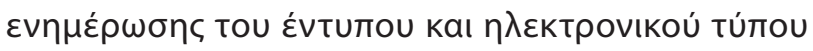

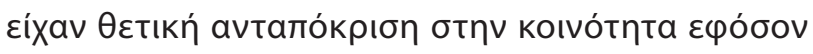

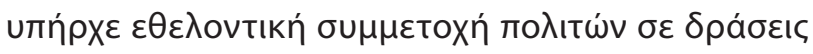

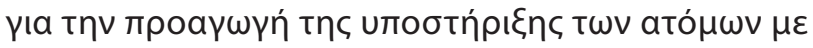

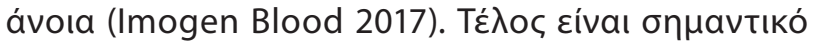

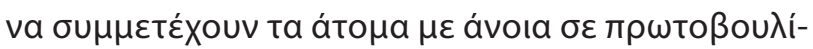

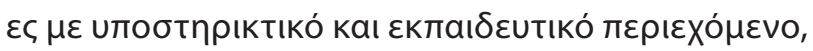

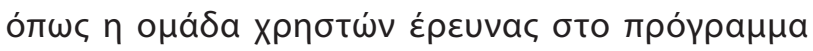
Sense-cog. ${ }^{26}$ 


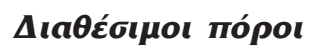

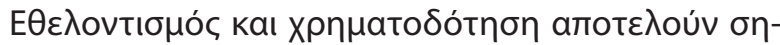

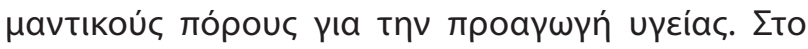

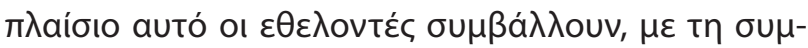

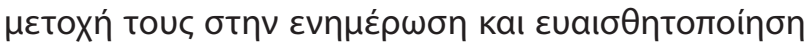

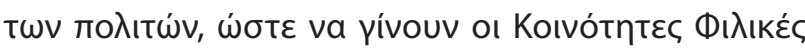

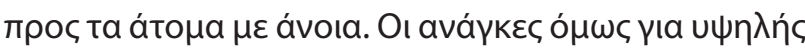

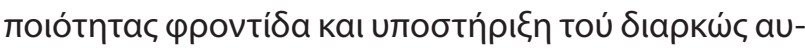

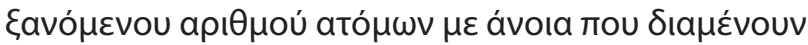

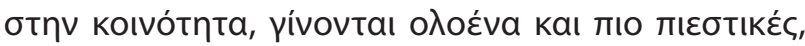

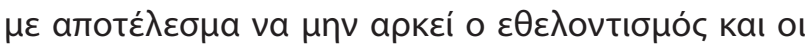

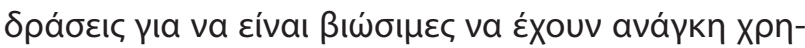

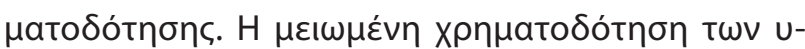

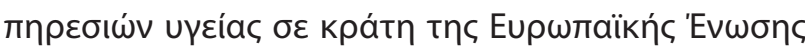

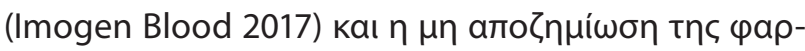

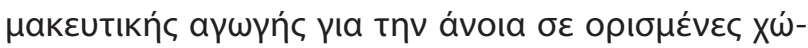

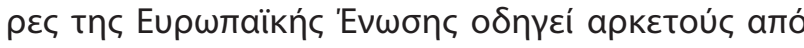

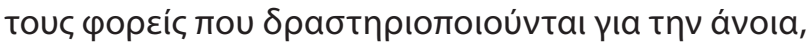

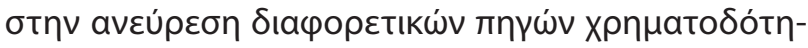

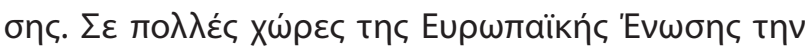

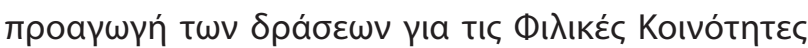

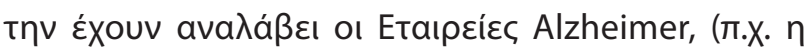

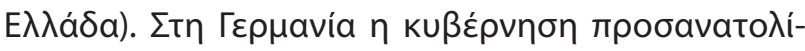

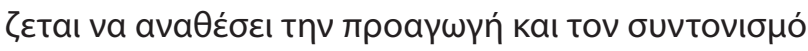

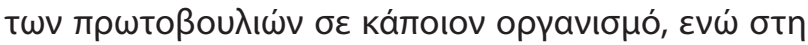

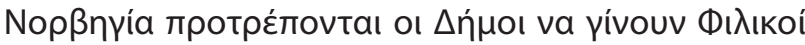

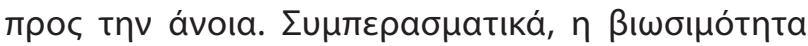

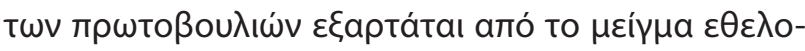

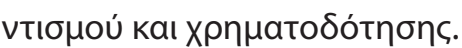

\section{Monítes}

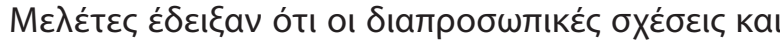

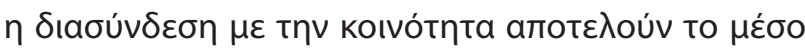

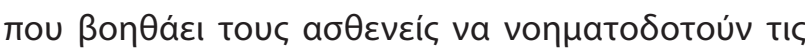

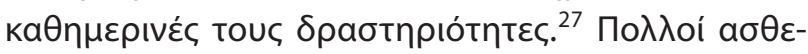

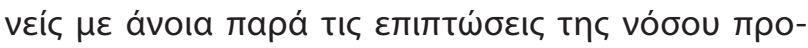

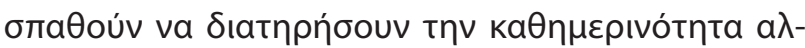

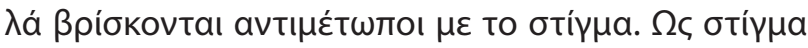

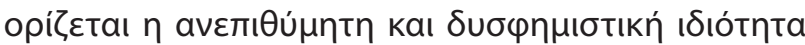

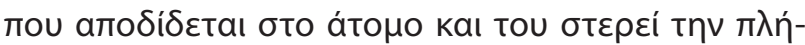

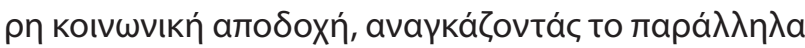

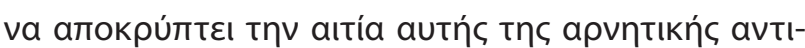

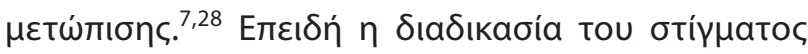

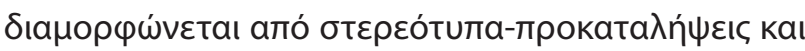

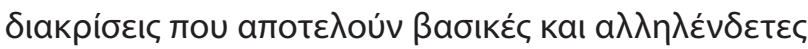

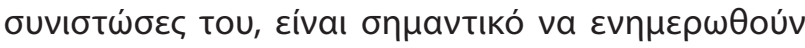

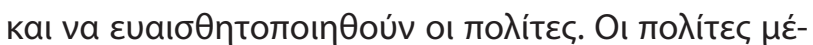

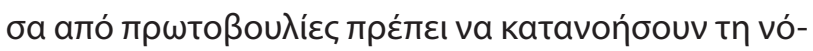

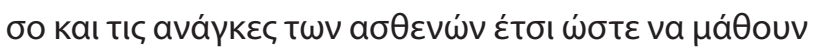

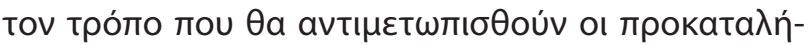

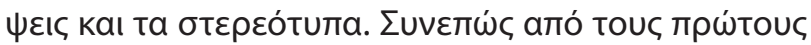

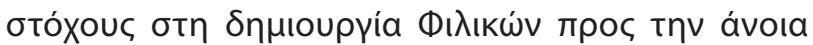

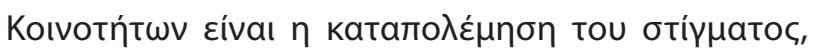

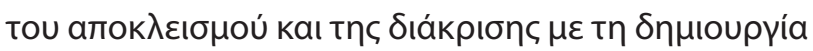

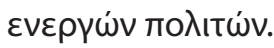

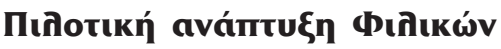 yıa tnv ávoı Kotvotńtwv

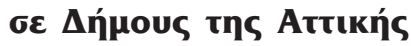

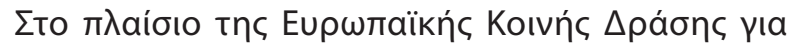

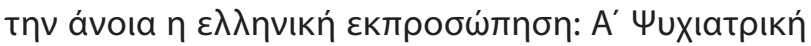

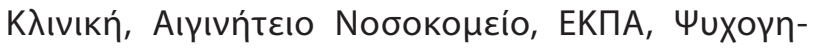

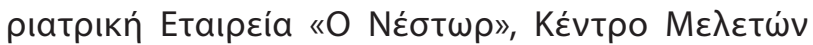

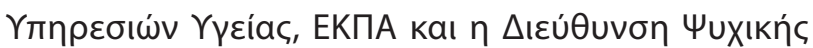

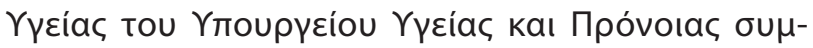

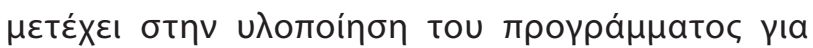

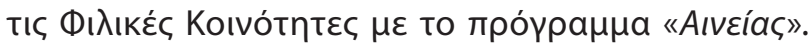

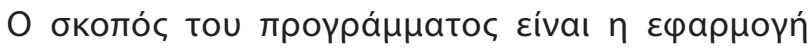

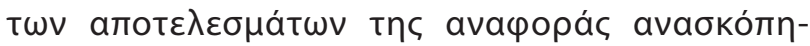

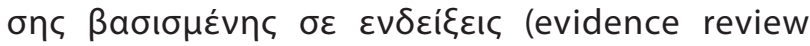

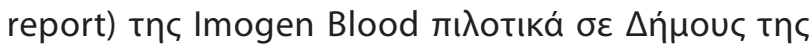

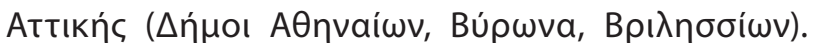

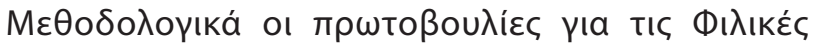

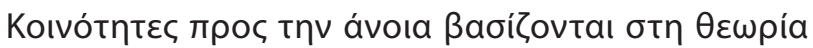

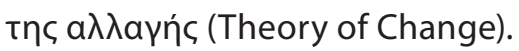

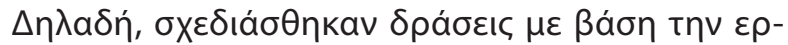

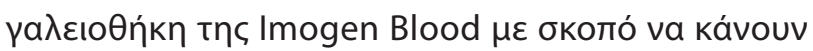

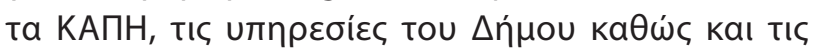

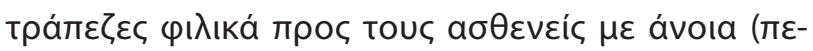

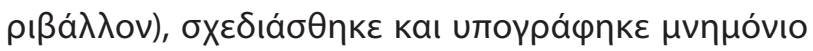

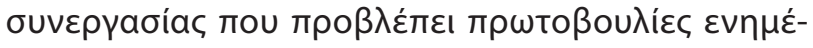

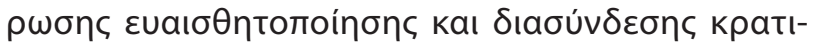

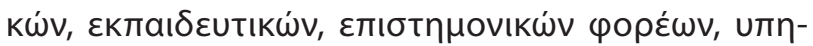

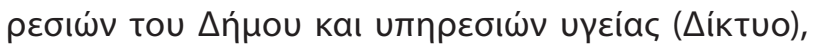

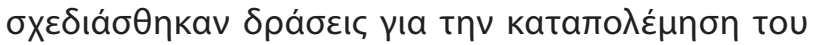

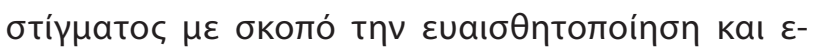

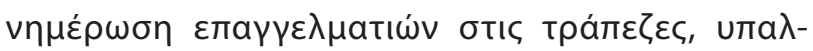

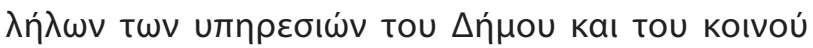

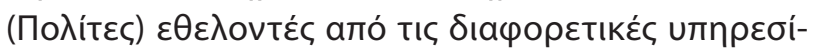

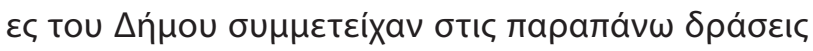

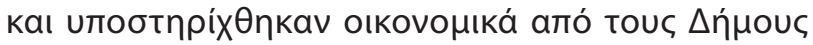

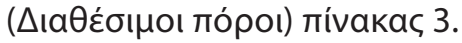




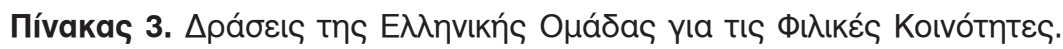

\begin{tabular}{|c|c|}
\hline 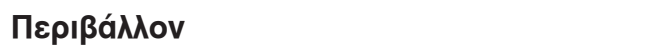 & Поגі́тєৎ \\
\hline Фıлıка́ КАПН & 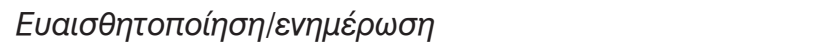 \\
\hline 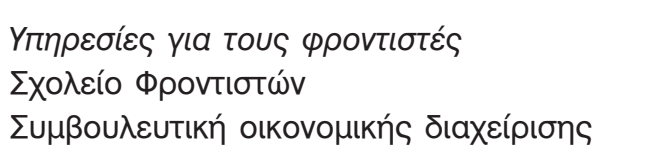 & 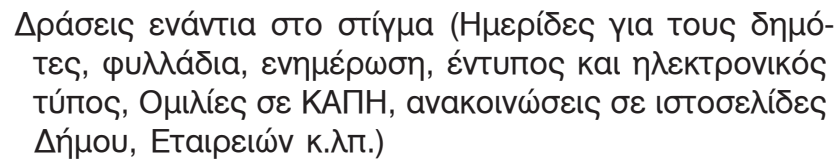 \\
\hline \multicolumn{2}{|l|}{ 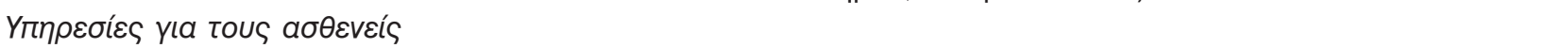 } \\
\hline \multicolumn{2}{|l|}{ 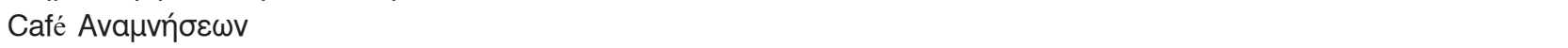 } \\
\hline \multicolumn{2}{|l|}{ 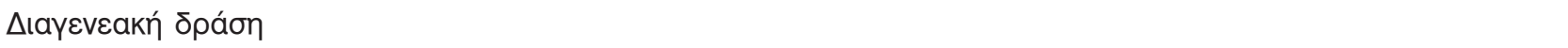 } \\
\hline \multicolumn{2}{|l|}{ 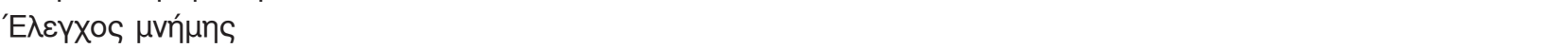 } \\
\hline \multicolumn{2}{|l|}{ 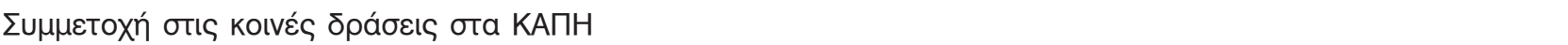 } \\
\hline \multicolumn{2}{|l|}{ 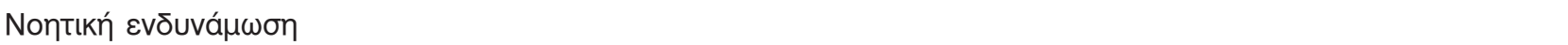 } \\
\hline \multicolumn{2}{|l|}{ 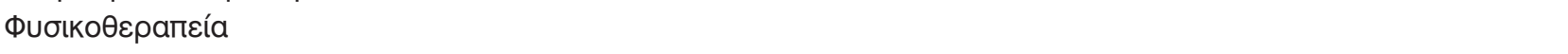 } \\
\hline \multicolumn{2}{|l|}{ 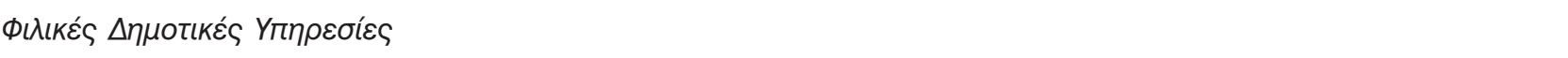 } \\
\hline \multicolumn{2}{|l|}{ 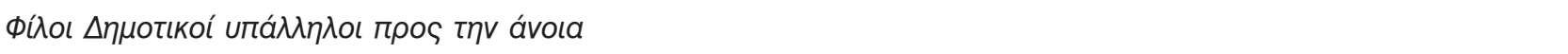 } \\
\hline \multirow{5}{*}{ 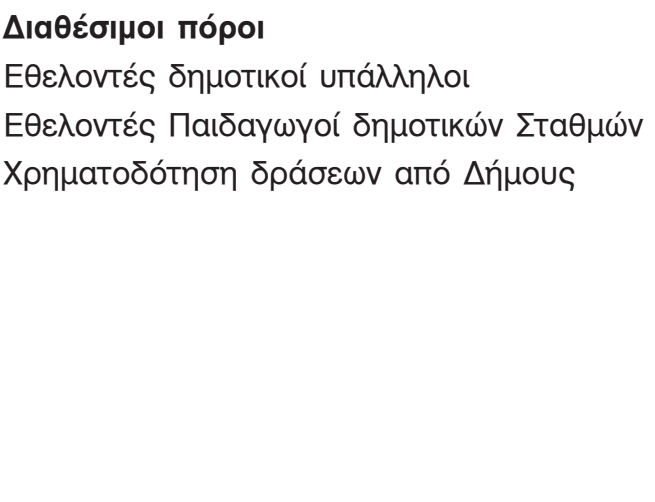 } & ¿íktua \\
\hline & 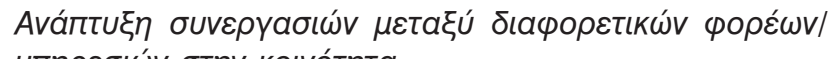 \\
\hline & 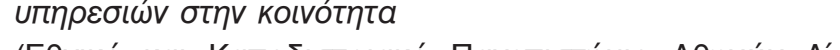 \\
\hline & 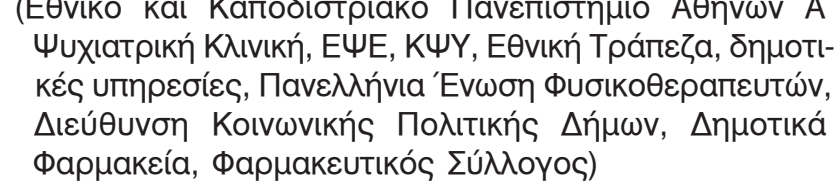 \\
\hline & 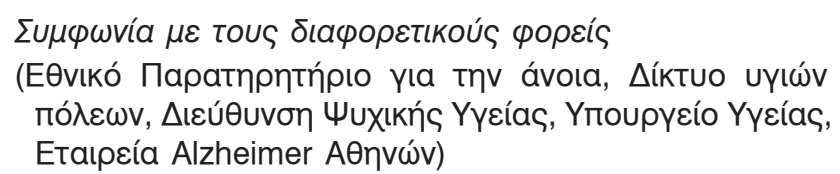 \\
\hline
\end{tabular}

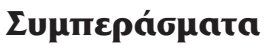

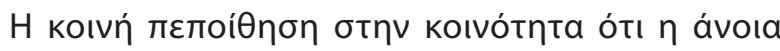

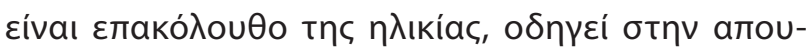

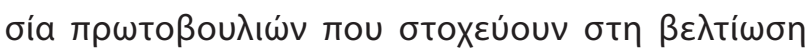

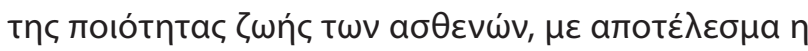

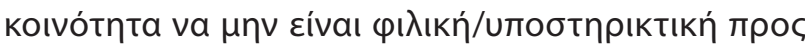

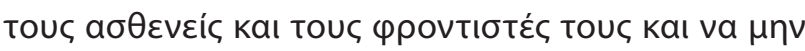

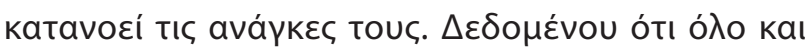

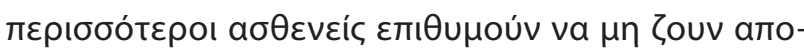

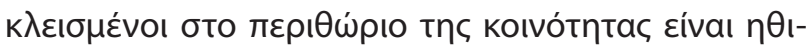

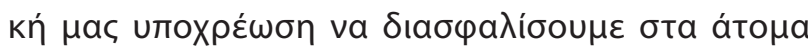

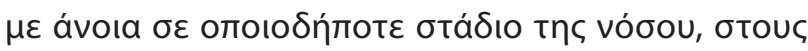

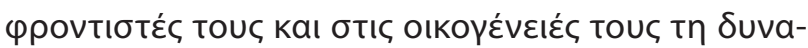

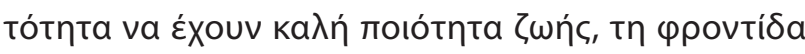

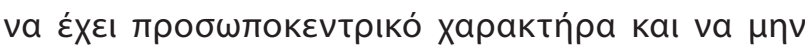

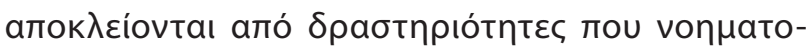

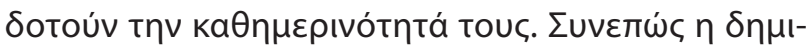

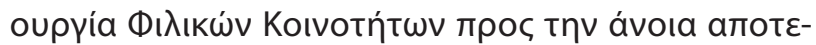

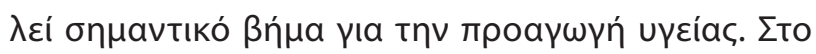

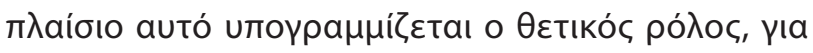

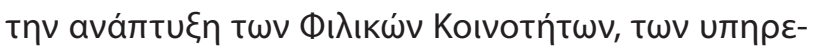

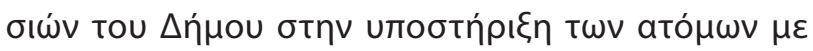

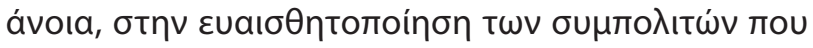

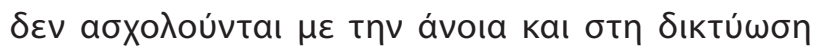

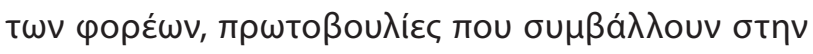

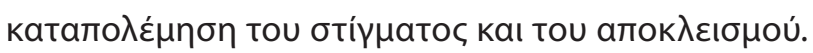

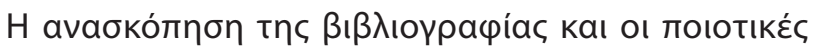

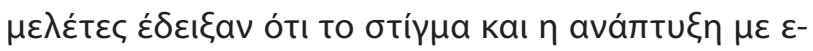

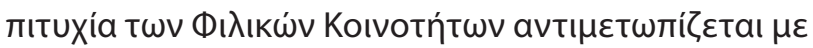

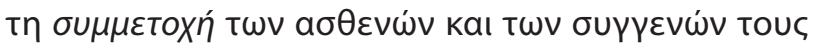

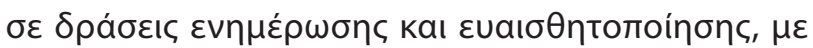

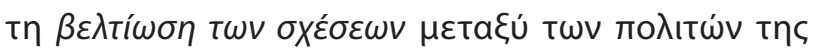

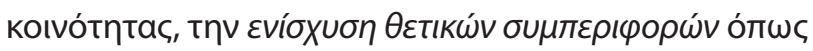

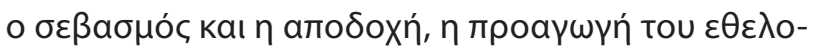

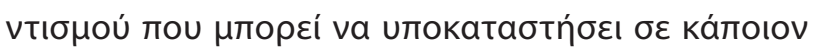




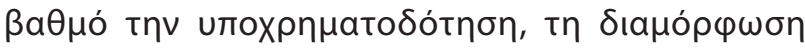

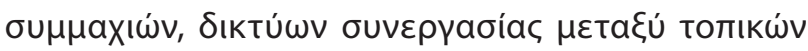

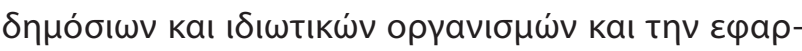

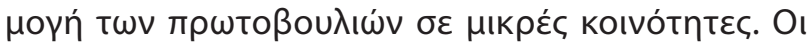

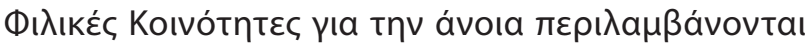

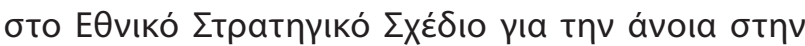

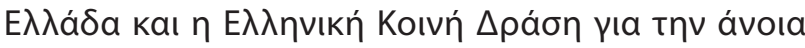

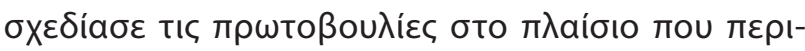

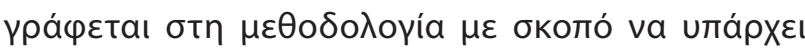

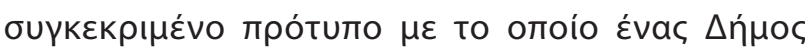

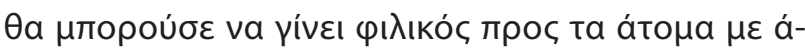

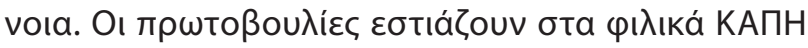

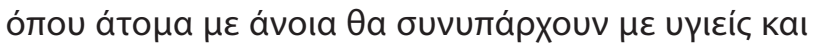

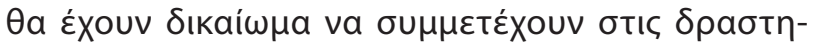

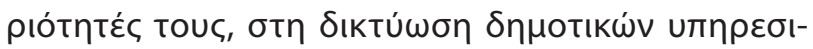

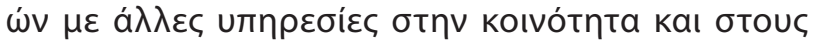

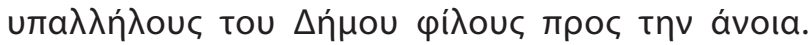

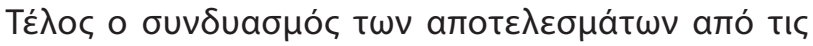

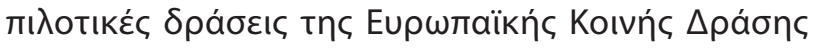

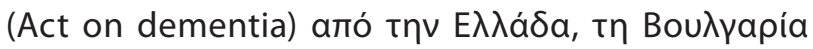

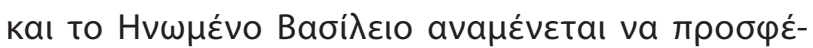

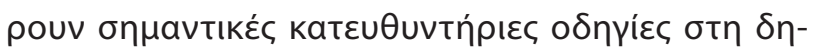

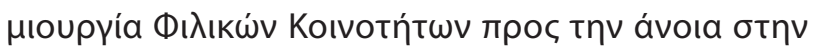

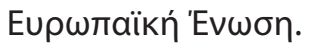

\title{
European Join Action on Dementia: Dementia Friendly Communities in Greece (DFC), the project "Aeneas"
}

\author{
A. Politis, ${ }^{1}$ A. Mougias, ${ }^{2}$ I. Petroulia, ${ }^{3}$ I. Smyrni, ${ }^{2}$ I. Tountas, ${ }^{3}$ \\ D. Ploumpidis, ${ }^{4}$ M. Mantzorou, ${ }^{5}$ E. Koukia, ${ }^{6}$ D. Kiosses, ${ }^{7}$ Ch. Papageorgiou, ${ }^{1}$ \\ DFC Working group ${ }^{8}$ \\ ${ }^{1} 1$ st Department of Psychiatry, National and Kapodistrian University of Athens, Athens, \\ ${ }^{2}$ Hellenic Association of Geriatrics and Gerontology, \\ ${ }^{3}$ Center For Health Services Research, National and Kapodistrian University of Athens, Athens, \\ ${ }^{4}$ Hellenic Psychiatric Association, \\ ${ }^{5}$ Department of Nursing, Faculty of Health and Caring Sciences, Athens, \\ ${ }^{6}$ Department of Nursing, National and Kapodistrian University of Athens, Athens, Greece, \\ ${ }^{7}$ Weill Cornell Institute of Geriatric Psychiatry, New York, USA, \\ ${ }^{8}$ M. Arsanoglou, M. Dimitriou, K. Kollia, V. Vaggeli, V. Panagiotakopoulou, E. Sifakaki, M. Loule, \\ P. Soranidou, A. Papadopoulou, E.S. Zaharopoulou, M. Stratigaki
}

Psychiatriki 2020, 31:82-90

The Health Care system has a vital role in improving services and support people with dementia, but it cannot prevent fear and lack of understanding of dementia. Lack of awareness and poor understanding in communities has a major impact on the experience of people with dementia and their caregivers. "Dementia Friendly Communities" is a program of EU as part of the Act on Dementia Joint Action, in order to advance common recommendations for the development of better services for people with dementia, which will improve their quality of life. It has been proposed that within a "Dementia friendly Community, people with dementia will not be marginalized and will be respected by their fellow citizens. The citizens, the public organizations and private sector enterprises cooperate in order to eliminate the obstacles which exclude dementia patients and their 
caregivers from participating in the life of their community". The Dementia Friendly Communities involve four fields: the environment (places), the citizens (people), the networks and the resources. Based on this model, pilot actions have been developed in Greece (Municipalities of Athens, Byron and Vrilissia), Bulgaria and UK and it is expected to provide important guidelines for the development of Dementia Friendly Communities in EU. In Greece the goal of the Join Action on Dementia was to promote the development of strong alliances within the local communities (municipalities) and the networking of organizations (State, Municipalities, NGOs, enterprises etc) with dementia patients and their caregivers and to educate more people in the municipalities in order to fight stigma and understand dementia.

Key words: Dementia Friendly Communities, dementia, caregivers, stigma, European Join Action.

\section{BıBAtoypacpía}

1. Wimo A, Jonsson L, \& Gustavsson A. Cost of illness and burden of dementia - The base option. Alzheimer's Europe 2008

2. Kosmidis $\mathrm{MH}$, Vlachos GS, Anastasiou CA, Yannakoulia M, Dardiotis E, Hadjigeorgiou G et al. Dementia Prevalence in Greece: The Hellenic Longitudinal Investigation of Aging and Diet (HELIAD). Alzheimer Dis Assoc Disord 2018, 32:232-239, PMID:29528855

3. Mougias AA, Politis A, Mougias MA, Kotrotsou I, Skapinakis P, Damigos D, Mavreas VG. The burden of caring for patients with dementia and its predictors. Psychiatriki 2015, 26:28-37, PMID: 25880381

4. Kaitelidou D, Kalogeropoulou M, Mougias A, Galanis P, Kontodimopoulos N, Papadopoulou P et al. Socio-Economic impact of Alzheimer's disease in Greece: Pilot study. Nurs Care Research 2013, 35:72-82, doi: 10.1016/j.jval.2013.08.1394

5. Petersen RC. Mild cognitive impairment as a diagnostic entity. J Intern Med 2004, 256:183-194, PMID:15324362

6. O'Rourke HM, Duggleby W, Fraser KD, Jerke L. Factors that Affect Quality of Life from the Perspective of People with Dementia: A Metasynthesis. J Am Geriatr Soc 2015, 63:24-38, PMID: 25597556

7. Von Kutzleben M, Schmid W, Halek M, Holle B, Bartholomeyczik S. Community-dwelling persons with dementia: what do they need? What do they demand? What do they do? A systematic review on the subjective experiences of persons with dementia. Ag Ment Health 2012, 16:378-390, PMID: 22250961

8. Hebert C, Scales K. Dementia friendly initiatives: A state of the science review. Dementia 2017, 1:1471301217731433, PMID: 28933191

9. Williamson T. My Name is not Dementia. Alzheimer's Society, London, 2010

10. Fortinsky $\mathrm{RH}$, Downs M. Optimizing person-centered transitions in the dementia journey: A comparison of national dementia strategies. Health Affairs 2014, 33:566-573, PMID: 24711316

11. Kane RA, Cutler LJ. Re-imagining long-term services and supports: Towards livable environments, service capacity, and enhanced community integration, choice, and quality of life for seniors. Gerontologist 2015, 55:286-295, PMID: 26035605
12. Parke B, Boltz M, Hunter K F, Champers T, Wolf-Ostermann K, Adi $\mathrm{MN}$ et al. A scoping literature review of dementia-friendly hospital design. The Gerontologist 2017, 57:e62-e74, PMID: 27831481

13. Shih-Yin Lin. Dementia-friendly communities and being dementia friendly in healthcare settings Curr Opin Psychiatry 2017, 30: 145-150, PMID: 26035599

14. Alzheimer's Europe. Dementia in Europe Yearbook 2013: with a focus on national policies relating to the care and support of people with dementia and their carers, as well as the prevalence of dementia including the Alzheimer Europe Annual Report 2012, Alzhemer's Europe, Luxembourg [online] http://www.alzheimer-europe.org/Publications/Dementia-inEurope-Yearbooks

15. Carers UK. Building Carer Friendly Communities: Research report for Carers Week 2016, Carers UK, London, (online) https://www.carersuk.org/for-professionals/policy/policylibrary/building-carer-friendly-communities-research-report-forcarers-week-2016

16. Reid S, Waterton J, Wild A. Attitudes to Dementia: Scottish Social Attitudes. NatCen Social Research, London, 2015

17. Philipson L, Hall D, Cridland E. Dementia-Friendly Kiama Pilot Project: Final Evaluation Report. University of Wollongong, New South Wales, 2016

18. Henwood M. Living a normal life: Supporting the development of Dementia Friendly Communities. Skills for Care, Leeds, 2015

19. Seydak E. Building a Dementia Friendly Community in Northern Ireland: Learning from the DEED Project in Derry, Joseph Rowntree Foundation, York, 2015

20. Robertson J, Evans D. Evaluation of a workplace engagement project for people with younger onset dementia. J Clin Nurs 2015, 24:2331-2339, PMID: 25926419

21. Wegleitner K, Heimerl K, Kellehear A (eds) Compassionate Communities: Case studies from Britain and Europe. Routledge, London and New York, 2016

22. Phinney A, Kelson E, Baumbusch J, O'Connor D, Purves B. Walking in the neighbourhood: Performing social citizenship in dementia. Dementia 2016,15:381-394, PMID: 27170588 
23. Crampton J, Dean J, Eley R. Creating a Dementia Friendly York (Internet). York: Joseph Rowntree Foundation; 2012. Available from: https://www.jrf.org.uk/report/creating-dementia-friendlyyork

24. Phillips R, Evans B, Muirhead S. Curiosity, place and wellbeing: encouraging place-specific curiosity as a "way to wellbeing". Environm Plann 2015, 47:2339-2354, PMID: 29445248

25. Mitchell L, Burton E, Raman S, Blackman T, Jenks M, Williams K. Making the outside world dementia-friendly: design issues and considerations. Environment and Planning B: Plann Design 2003, 30:605-632, doi:10.1068/b29100

26. Regan J, Frison E, Collin F, Dawes P, Hann M, Himmelsbach I et al. Individualised sensory intervention to improve quality of life in people with dementia and their companions (SENSECOG)trial): study protocol for a randomized controlled trial. Trials 2019, 25:80, PMID: 30683150

27. Smith K, Gee S, Sharrock T, Croucher M. Developing a dementia-friendly Christchurch: perspectives of people with dementia. Austr J Ag 2016, 35:188-192, PMID: 27061350
28. Economou M, Richardson C, Gramandani C, Stalikas A, Stefanis C. Knowledge about schizophrenia and attitudes towards people with schizophrenia in Greece. Intern J Soc Psychiatry 2009, 55:361-371, PMID: 19553365

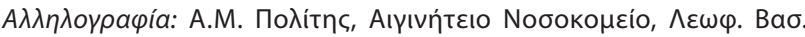

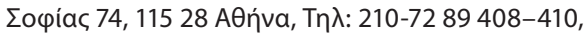

e-mail:apolitis@med.uoa.gr 